$\mathrm{AD}$

(Leave blank)

\begin{abstract}
Award Number:
w81XWH-10-1-0483

TITLE :

PRINCIPAL INVESTIGATOR:

Andrew J. Armstrong, MD SCM

CONTRACTING ORGANIZATION:

Duke University

Durham, NC 27705-4677

REPORT DATE:

July 2014
\end{abstract}

"Epithelial Plasticity in Castration-Resistant Prostate Cancer: Biology of the Lethal Phenotype"

TYPE OF REPORT:

Annual Summary

PREPARED FOR: U.S. Army Medical Research and Materiel Command Fort Detrick, Maryland 21702-5012

DISTRIBUTION STATEMENT:

X Approved for public release; distribution unlimited

The views, opinions and/or findings contained in this report are those of the author(s) and should not be construed as an official Department of the Army position, policy or decision unless so designated by other documentation. 


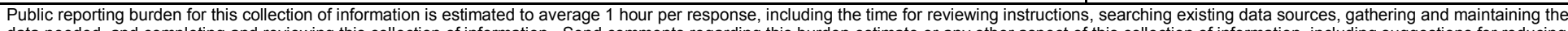

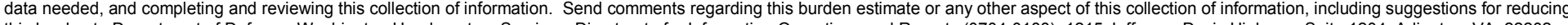

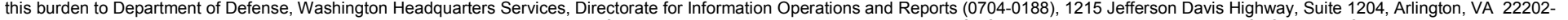

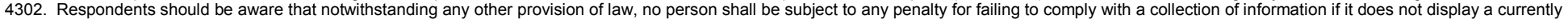
valid OMB control number. PLEASE DO NOT RETURN YOUR FORM TO THE ABOVE ADDRESS.

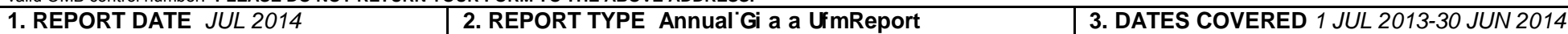

\section{TITLE AND SUBTITLE}

"Epithelial Plasticity in Castration-Resistant Prostate Cancer Biology of the Lethal Phenotype"

5a. CONTRACT NUMBER

5b. GRANT NUMBER

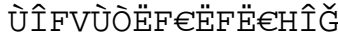

5c. PROGRAM ELEMENT NUMBER

\section{AUTHOR(S)}

Andrew J Armstrong, MD ScM

email: andrew.armstrong@duke.edu 5d. PROJECT NUMBER

5e. TASK NUMBER

5f. WORK UNIT NUMBER

8. PERFORMING ORGANIZATION REPORT NUMBER

Duke University Medical

Center

Durham, NC 27705

9. SPONSORING / MONITORING AGENCY NAME(S) AND ADDRESS(ES)

US Army Medical Research

and Materiel Command

Fort Detrick, Maryland 21702- 5012
10. SPONSOR/MONITOR'S ACRONYM(S)

11. SPONSOR/MONITOR'S REPORT NUMBER(S)

\section{DISTRIBUTION / AVAILABILITY STATEMENT}

Approved for public release; distribution unlimited

\section{SUPPLEMENTARY NOTES}

\section{ABSTRACT}

The purpose of this DOD PRTA is to investigate the role of epithelial plasticity in the promotion of metastasis in advanced prostate cancer through a sequential interrogation of biomarkers in primary prostate tumors, metastases, and circulating tumor cells (CTCs). In this 20132014 annual report, I provide updates on our investigation of EMT biology in both localized and metastatic prostate cancer, with particular emphasis on CTC biology as a biomarker of disease progression and metastasis. I describe our ongoing investigation into novel CTC phenotypes in men with metastatic castration-resistant prostate cancer (CRPC) using OB-and N-cadherin and c-met capture of CTCs, investigation of plasticity biomarkers in localized PC, and the investigation of CTCs for DNA and RNA biomarkers (copy number variations and RNA Sequencing) that may each shed light on the molecular pathophysiology of metastatic spread. We provide evidence for the common co-expression of epithelial and mesenchymal/EMT biomarkers in CTCs from patients with metastatic castration-resistant prostate cancer (CRPC) as well as the common expression of stem cell biomarkers in these CTCs. This data provides strong evidence for the importance of EMT to PC metastasis in humans, but the utility of assessing mesenchymal biomarkers in localized disease is limited based on our data, particularly using a tissue microarray based assessment. These data suggest that the majority of localized prostate cancers are epithelial in phenotype but that during hematogenous dissemination, a dual epithelial-mesenchymal phenotype emerges.

\section{SUBJECT TERMS}

Circulating tumor cells, prostate cancer, epithelial plasticity, epithelial mesenchymal transition, metastasis, lethal phenotype, biomarkers, training award

\section{SECURITY CLASSIFICATION OF:}

\begin{tabular}{|l|l|l|}
\hline $\begin{array}{l}\text { a. REPORT } \\
U\end{array}$ & $\begin{array}{l}\text { b. ABSTRACT } \\
U\end{array}$ & $\begin{array}{l}\text { c. THIS PAGE } \\
U\end{array}$ \\
\hline
\end{tabular}

17. LIMITATION OF ABSTRACT

UU

\begin{tabular}{|c|l|}
$\begin{array}{l}\text { 18. NUMBER } \\
\text { OF PAGES }\end{array}$ & $\begin{array}{l}\text { 19a. NAME OF RESPONSIBLE PERSON } \\
\text { USAMRMC }\end{array}$ \\
\cline { 2 - 2 } 184 & $\begin{array}{l}\text { 19b. TELEPHONE NUMBER (include area } \\
\text { code) }\end{array}$ \\
\end{tabular}




\section{Table of Contents}

\section{Page}

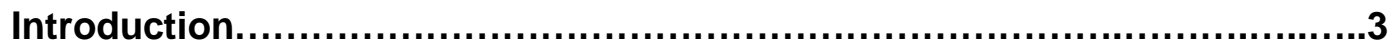

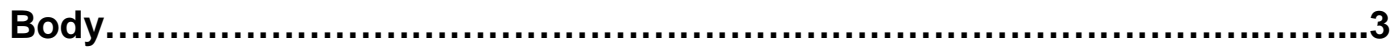

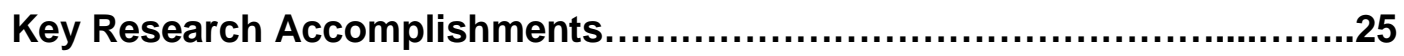

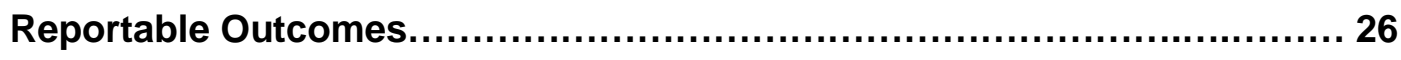

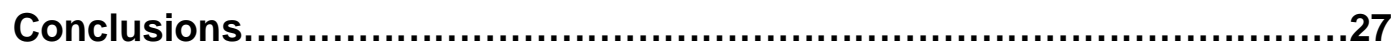

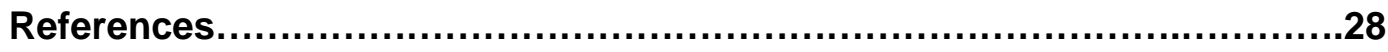

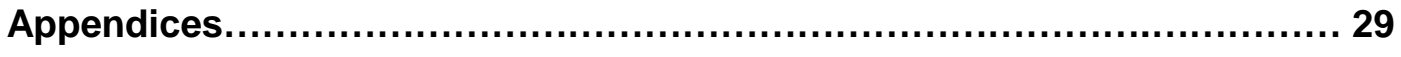




\section{INTRODUCTION}

In 2014, over 80 U.S. men will die every day from metastatic prostate cancer (PC) ${ }^{1}$. Many deaths could potentially be prevented or delayed through identification and treatment directed at high risk disease. Currently, clinical/pathologic measures (i.e. PSA, stage, grade) provide no biologic insights into the process by which PC cells metastasize and become lethal. The measurement of circulating tumor cells (CTCs) in men with PC represents one biomarker with prognostic and predictive implications ${ }^{2}$. Many patients with metastatic PC, however, have undetectable CTCs, limiting clinical utility. We have identified epithelial-mesenchymal transitions (EMT) in experimental models of PC in which the cellular phenotype undergoes reversible (plastic) changes from an epithelial to a mesenchymal nature facilitating metastatic spread, followed by epithelial reversion in the target metastatic organ ${ }^{3}$. While in the active process of metastasis, CTCs may possess a mesenchymal/plastic phenotype, and thus may not be captured by existing epithelial-based CTC technologies. In this PRTA 2013-2014 annual report, we provide updates on our investigation of EMT biology in localized prostate cancer as a biomarker of recurrence, and in men with metastatic prostate cancer, with particular emphasis on circulating tumor cell biology as a biomarker of disease progression and metastasis. We also describe our ongoing investigation into novel CTC phenotypes in men with metastatic castration-resistant prostate cancer (CRPC), EMT biomarker studies in localized PC, and the investigation of CTCs for DNA biomarkers (copy number variations and whole exome sequencing) that may each shed light on the molecular pathophysiology of metastatic spread. We provide evidence for the common co-expression of epithelial and mesenchymal/EMT biomarkers in CTCs from patients with metastatic castration-resistant prostate cancer (CRPC) as well as the common expression of stem cell biomarkers in these CTCs ${ }^{4}$. This data provides strong evidence for the importance of epithelial plasticity to prostate cancer metastasis in humans, particularly during castration resistant progression. We have also identified and optimized methods for rare cell whole genome amplification and exome sequencing of CTCs in real time from patients, in which common genomic aberrations found in metastatic lesions can be identified in CTCs. Together these aims will provide insight into metastasis biology in PC and lead to the identification of relevant targets for therapies directed against this lethal metastatic process.

\section{BODY}

Task 1: To link evidence of epithelial plasticity with adverse clinical features and PSA-based outcomes using a Durham VA Hospital prostate cancer tissue microarray and the SEARCH database (Months 1-36)

Key Research Accomplishments: See the years 1-3 annual reports for a description of our work to date related to Ki-67, vimentin, E- and N-cadherin. In year 4, we have added SNAIL and TWIST expression assessment. The findings are now summarized in a paper submitted in July 2014 to Clinical Cancer Research (Appendix A). Essentially, we have found that the majority of localized prostate cancers are epithelial in phenotype, and that mesenchymal biomarker expression in the bulk tumor in our cohort is not associated with PSA relapse after surgery. This may relate to the methods used (bulk tumor scoring by pathology rather than rare cell isolation or geographic descriptions of EMT biomarkers at the invasive front for example), limited power ( $<5 \%$ of men have died from prostate cancer over more than 10 years of follow up), and the overall good risk status of our patient cohort. Nevertheless, this data calls into question the clinical utility of EMT biomarker assessment using standard radical prostatectomy 
tissues. We did validate $\mathrm{Ki}-67$ as a prognostic biomarker in our series, highlighting the importance of proliferation and the epithelial phenotype in localized disease. Our data does not exclude the possibility of a link between rare cellular populations (ie stem-like mesenchymal cells) and dissemination/relapse that may be missed using these methods. See appendix for figures and tables and results and discussion. This work concludes Task 1.

Task 2: To identify the presence of mesenchymal and stem cell markers on circulating prostate cancer cells (CTCs) derived from men with castration-resistant prostate cancer and associated with adverse clinical outcomes (Months 1-18).

Key Research Accomplishments: We have successfully conducted this task, leading to the attached published manuscript (Armstrong et al, Mol Cancer Res 2011 ${ }^{4}$ ) describing in detail our findings of EMT and stem cell markers in the majority of CTCs from men with metastatic castration-resistant prostate cancer and women with metastatic breast cancer (see year 1 annual report). This data provided among the strongest and earliest evidence to date for the existence of EMT (epithelial-mesenchymal transition) in human cancer, particularly in circulating tumor cells. Given the importance of EMT to cancer metastasis and the lethal phenotype, as well as to the generation of stemness and chemoresistance, we believe these findings have profound implications for cancer biology. They suggest that EMT plays a strong role in prostate cancer metastasis during castration-resistant progression. The findings also suggest that our existing CTC assays may underdetect circulating tumor cells with more mesenchymal phenotypes, given that they are likely to have reduced or absent EpCAM expression. Please see manuscript for a detailed discussion of these implications and results.

The findings above led us to the development of additional novel capture ferrofluids based on EMT biology as part of an ongoing research collaboration between Duke University (PI Armstrong) and Janssen/Veridex. This is further described in Task 3.

As an exploratory project, we evaluated all of our CTC patients at Duke who enrolled in these studies to ask the question: is there a clinical phenotype of aggressive mCRPC associated with a low CTC count by the Cellsearch method? If so, perhaps this would indicate evidence of plasticity and loss of EpCAM and EMT in these patients. We hypothesized that men with mCRPC and visceral or high grade disease would have such a finding of a relatively low CTC count for their burden of disease. We also conducted a prognostic analysis of CTCs with survival and progression free survival. Results are attached in the appendix $\mathbf{B}$ as a paper submitted to Annals of Oncology (2014). Essentially, we could not identify a low CTC population in these men; men with visceral and/or high grade CRPC had higher CTCs than men with lower grade or non-visceral patients. While we cannot exclude that some CTCs may have been missed by the Cellsearch method, the absolute number of CTCs by Cellsearch increases as disease burden increases. The CTC enumeration provides independent prognostic value for survival and PFS in these men, even in the visceral subset of patients. While CTC enumeration was associated with elevations in PSA and alkaline phosphatase and LDH and lower hemoglobin, CTC enumeration provided independent clinical value for prognosis, both before therapy and over time during progression. The detection of non-EpCAM CTCs remains an area of priority work in task 3, however, given that the number of CTCs is likely to be higher in these poor prognostic patients, and given evidence that loss of epithelial biomarkers accompanies CRPC progression (see our recently published review on this topic in appendix C).

Thus, this task is complete.

Task 3: To refine the circulating tumor cell detection technology to capture circulating prostate tumor cells based on a mesenchymal surface marker rather than an epithelial marker (Months 37-60). This corresponds to pilot study 2 of SA2. 
Key Research Accomplishments: Based on our above findings and patent (see last year's annual report), we have an ongoing a collaborative research relationship with Veridex/Johnson and Johnson (now Janssen Pharmaceuticals) to develop a second generation CTC detection/capture assay based on task 2 above. These efforts are supported by the DOD PRTA (salary support). We have acquired a novel CTC detection instrument with a novel ferrofluid targeted at both $\mathrm{N}$ - and OB-cadherin rather than EpCAM. This work has led to the development of a patent focused on the novel detection of circulating tumor cells using an EMT antigenbased ferromagnetic capture method (submitted 9/24/10, international application number PCT/US10/50223). Preliminary results from this work involving both healthy volunteers and men with mCRPC was presented at ASCO 2012 (abstract 10533, see last year's annual report). We have completed enrollment for the OB-cadherin capture study of men with mCRPC and results were presented in the year 3 annual report and are not again summarized here.

The capture of novel CTCs expressing OB-cadherin in a subset of men with MCRPC raises the possibility of epithelial plasticity, given the clonal derivation of non-epithelial CTCs (ie lacking cytokeratin and expressing OB-cadherin) that are metastasis-initiating cells. These cells were not present in healthy volunteers when CD45 and CD31 were used as negative exclusion biomarkers. Figure 1 summarizes the findings in 24 men with mCRPC who were tested for the presence of novel CTCs using the OB-cadherin capture method and further characterized by beta-catenin expression as an EMT-independent protein (ie not CK based). Essentially, while we did identify a subset of men with OB-cadherin + CTCs, only $12 \%$ of men had $>5$ CTCs per $7.5 \mathrm{~mL}$ whole blood. While no healthy volunteers had any OB-cadherin+ cells that lacked CD45/CD31 expression, this low level of detection in bone metastatic CRPC patients has led to further efforts to identify and better characterize this rare subpopulation. Currently, we are modifying the OB-cadherin capture kit to remove the beta catenin characterization step, which may lead potentially to missing some CTCs that lack beta-catenin. Characterization of CTCs with CD45, CD31, DAPI, and a second OB-cadherin intracellular antigen is proposed as the next test kit, and is currently in development and will be tested in a cohort of men with mCRPC and healthy volunteers over year 5 of the grant.

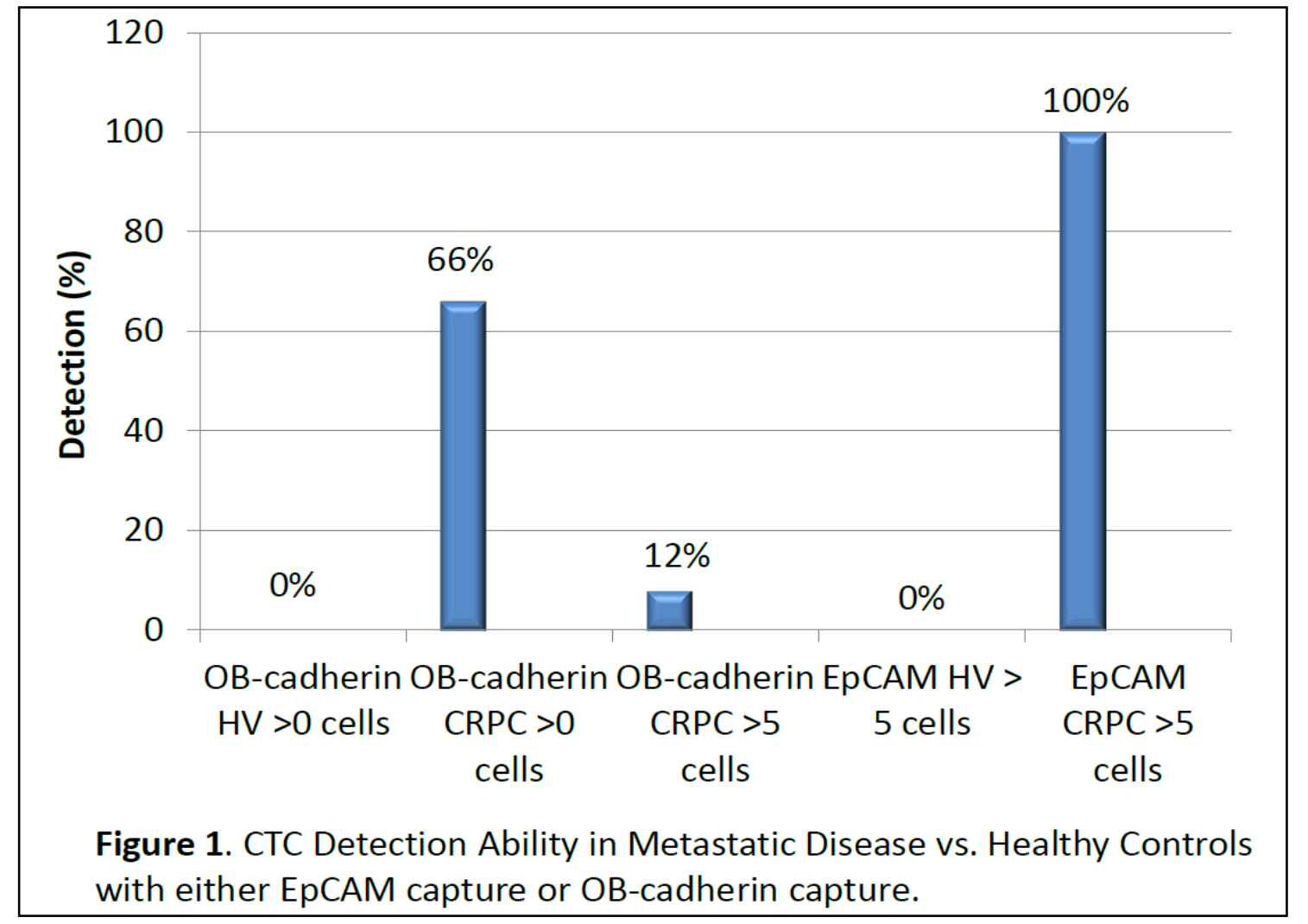


For $\mathrm{N}$-cadherin capture, we have explored a novel CTC capture reagent that captures CTCs that express $\mathrm{N}$-cadherin using an antibody ferrofluid directed against the extracellular domain of $\mathrm{N}$-cadherin. A second characterization step defines a CTC as being $\mathrm{N}$-cadherin positive based on intracellular $\mathrm{N}$-cadherin antigen detection, CD45 negative, and DAPI positive (figure 2).

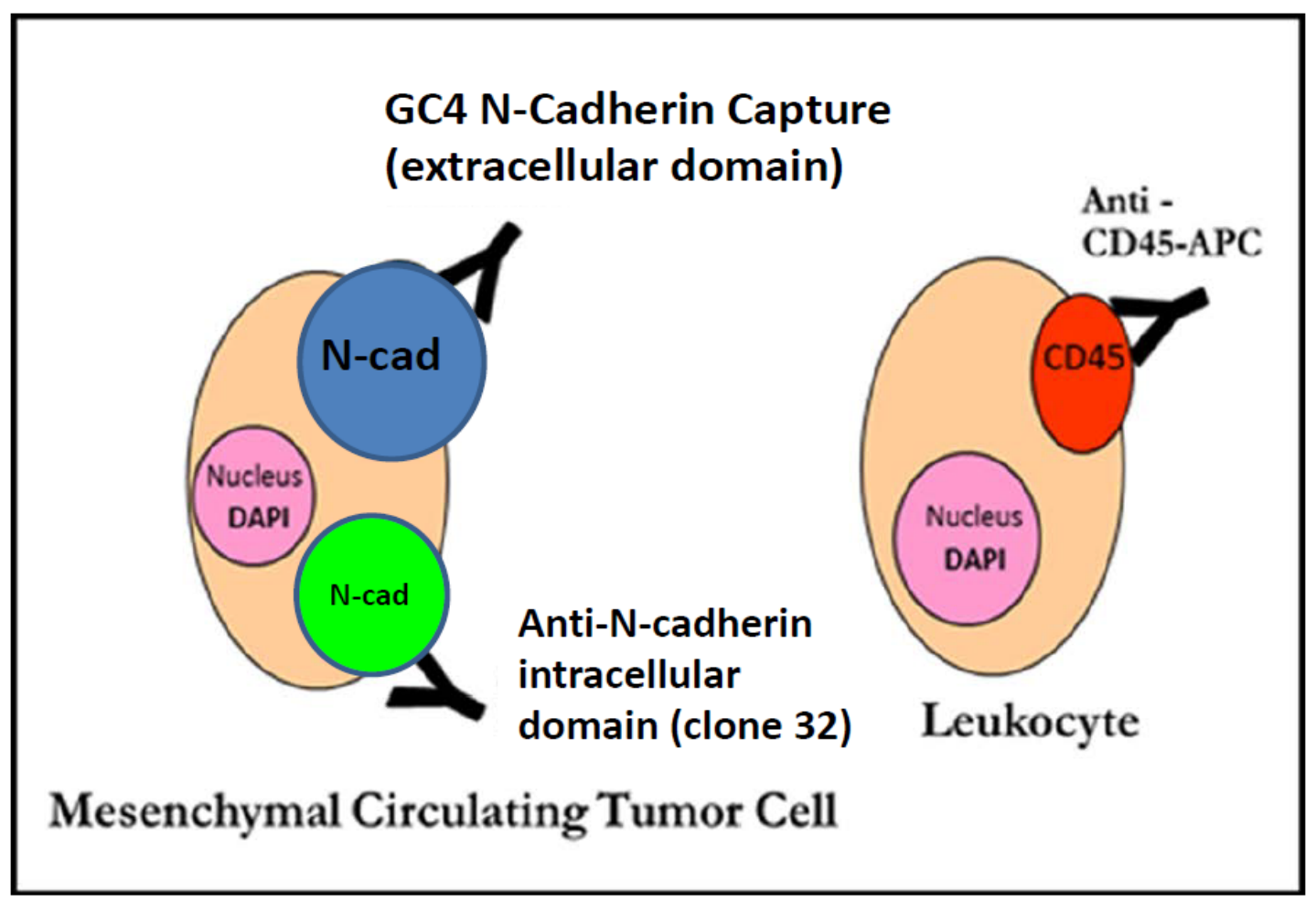

Figure 2. Defining a CTC based on $\mathrm{N}$-cadherin capture.

In spiking studies of 1000 positive control cells (PC-3 cells) into healthy volunteer blood, a percent yield of $\sim 100 \%$ was obtained, indicating excellent sensitivity but some nonspecific binding, given that $\mathrm{N}$-cadherin is not uniformly expressed by all PC-3 cells. With negative control cells (SKBR cells), no CTCs were identified after spiking of 1000 cells, and thus minimal nonspecific binding was seen. In 7 healthy volunteer samples (unspiked), no CTCs were identified using this $\mathrm{N}$-cadherin dual detection method, indicating a very low level of noise/nonspecific signal.

Given these encouraging results in spiking studies and normal healthy volunteers indicating detection of the intended cells but no detection of cells in non-cancer patients, we proceeded to test the N-cadherin capture method in 16 men with mCRPC (some duplicate samples) progressing on their most recently treatment. While the EpCAM standard Cellsearch kit was able to detect CTCs in the majority of men (range 0-280 cells, mean 40 cells, median 10 cells). For $\mathrm{N}$-cadherin capture, the range was $0-1$ cells (mean 0.13 , median 0 cells). An example of an $\mathrm{N}$-cadherin + CTC is shown in Figure 3. However, significant issues related to leukocyte contamination and low detection rates in men with mCRPC have led to plans to change this novel ferrofluid before further testing. 


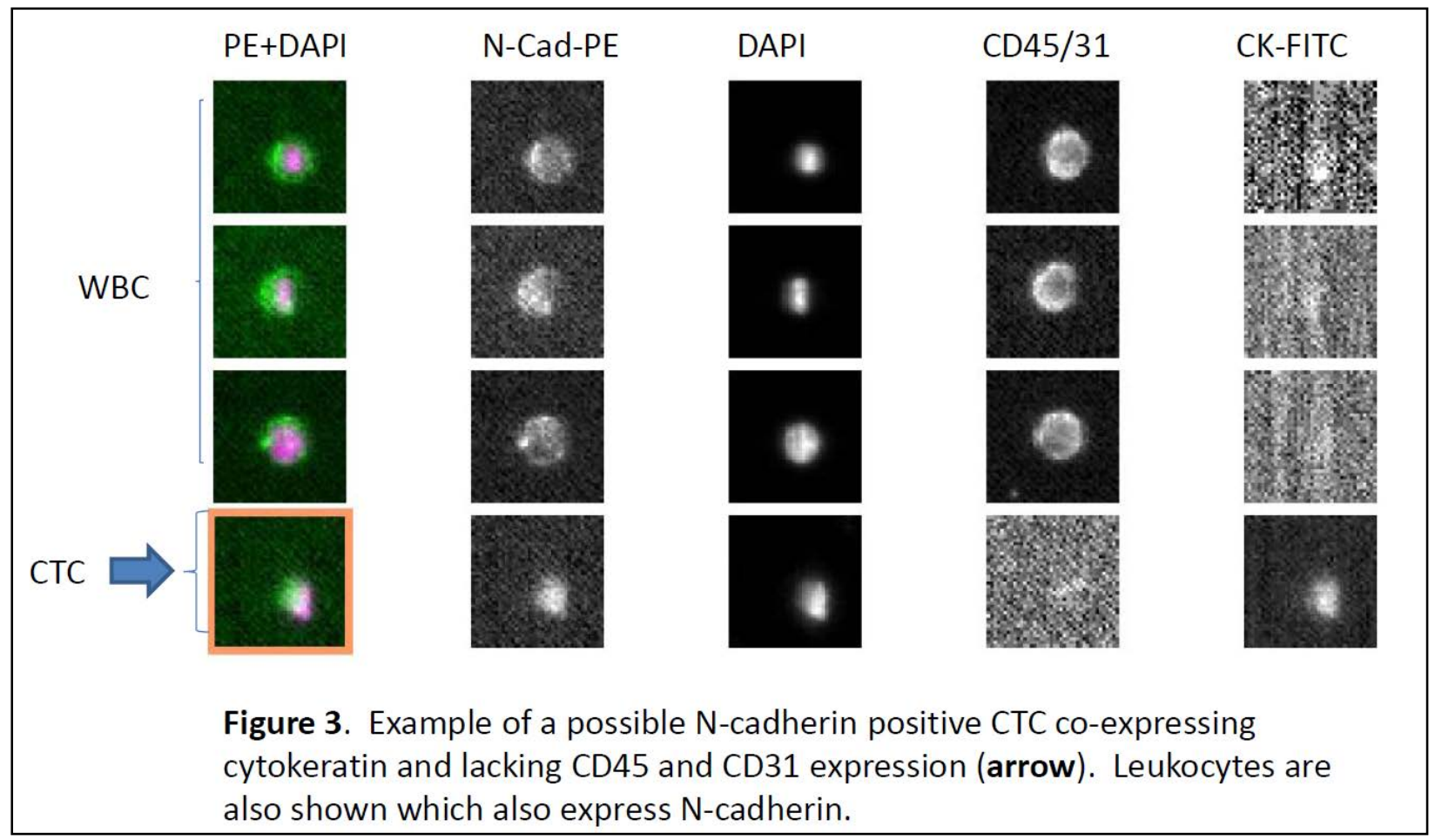

In year 5, we will test a newly modified version of both OB- and N-cadherin capture reagents for novel CTC detection, which will include use of the extracellular domain for capture of each antigen (two separate CTC kits will be explored in parallel) and use of the intracellular domain for characterization. Cells expressing either CD45 (leukocytes) or CD31 (endothelial cells) will be excluded based on our prior work (see year 3 summary report) showing that a subset of human endothelial cells co-express OB-cadherin and CD31. We will pilot this novel ferrofluid kit in healthy volunteers to establish a threshold for detection and in spiked positive control cell lines in human blood. Following the successful testing in normal individuals and with spiked samples, we will investigate the performance of each ferrofluid in a cohort of 10 men with $\mathrm{mCRPC}$ in parallel.

Finally, while not within the scope of the current PRTA, this work has additionally led to the codevelopment of a novel CTC ferrofluid to capture cells based on overexpression of the c-met oncogene. C-met is commonly amplified or overexpressed by a range of solid tumors including GI and GU cancers (colorectal, gastric, prostate, renal, bladder), and has been linked to invasive behavior, aggressive disease, and metastatic and resistant outgrowth as well as epithelial plasticity. Thus, this PRTA has been leveraged for the successful study and adaptation of a novel ferrofluid as part of the Duke-Janssen collaboration to test c-met capture in a cohort of patients with metastatic GI and GU cancers. We have shown that this test performs well with spiked samples of c-met amplified cells and does not detect cells from healthy volunteers. Further data on this novel capture will be conducted during year 5 of this grant.

Task 4: To detect similarities in RNA expression profile and patterns of oncogenic expression in circulating tumor cells and matched metastatic prostate cancer tumor tissue (Months 1-54).

Key research accomplishments: The major obstacle to performing RNA expression analysis from CTCs taken from patients with CRPC has been the isolation of a pure population of cells from whole blood that is devoid of leukocytes. Through a prolonged trial and error process intended to optimize this methodology, we have begun to have success in isolating these pure cellular populations. Our current operating procedures include drawing blood into EDTA vacutainers, followed by an initial red cell lysis followed by CD45 magnetic bead negative exclusion of contaminating leukocytes. This enriched CTC population is then further enriched 
and purified through FACS, gating against a tumor antigen (i.e. EpCAM) and negative excluding CD45 a second time. There is a high degree of CTC loss in this process; however, we are currently able to isolate a pure population of about $10-30 \%$ of the original spiked CTC population in simulated runs, which produces sufficient numbers of cells for RNA Sequencing. The methods for performing and testing RNA Seq on CTCs are described in last year's annual report. This work has identified the feasibility of RNA Sequencing of rare cells down to 10-20 cells spiked into whole blood, in which RNA expression analyses were similar with 50 cells as compared to 500 cells, for example, and were similar to the pure cell line sequencing data. Please refer to last year's summary for details and results.

A grant to the Prostate Cancer Foundation Global Treatment Sciences Challenge Award was submitted in May 2014 for funding to support both DNA and RNA biomarker studies of CTCs in men with mCRPC in the context of approved therapies such as abiraterone acetate, enzalutamide, and docetaxel chemotherapy. This grant was awarded in July 2014 (funding start date to be determined), and will support further work on the molecular characterization of CTCs in men with mCRPC treated with these systemic therapies. A panel of EMT biomarkers defined by RNA expression will be evaluated in this proposed grant from circulating tumor cell enriched blood, and correlated with alterations in AR biology (mutations, splice variants, loss of AR function) and neuroendocrine prostate cancer development. The EMT genes identified as differentially expressed during year 3 of the current grant will be evaluated in this proposed new award. This new proposal and study will start in the fall of 2014 across multiple DOD Prostate Cancer Clinical Trial Consortium (PCCTC) sites with Duke being the lead site, and include RNA biomarkers particularly AR-v7 biomarkers using the Johns Hopkins AR-v7 Adnatest biomarker, the EPIC Sciences AR-v assay, and the Cornell AR-v multiplex RT-PCR assay, in addition to RNA-Sequencing of CTC collected from men with mCRPC prior to and during progression on enzalutamide and abiraterone acetate and taxane chemotherapy. The objective of this Challenge Grant is to develop a CTC-based molecular predictor of efficacy of novel hormonal therapy and taxane therapy and to characterize the biology of mCRPC using a CTC-based biomarker approach. Thus, we have leveraged this DOD PRTA funding of preliminary data around CTC-based RNA studies to obtain a larger multicenter clinical grant to test a range of CTC-based RNA and DNA biomarkers in men with mCRPC.

Task 5: To estimate the clonality and heterogeneity of circulating tumor cells as compared with metastatic sites by DNA array-based comparative genomic hybridization (Months 1-54).

Key Research Accomplishments: Please refer to the year 3 annual report. Following the successful completion of array-based comparative genomic hybridization (arrayCGH) studies of CTCs from men with mCRPC, we have continued to investigate arrayCGH in a larger group of men. In addition, we have performed whole exome sequencing of CTCs now from men with $\mathrm{mCRPC}$. We believe that we have now optimized the methods for CTC isolation using RBC lysis, CD45 depletion, and flow sorting, followed by DNA isolation and WGA. These methods are described below.

After red cell lysis and CD45 depletion using Dynabeads, circulating tumor cells (CTC) are isolated from in a FACS cell sorter using EpCAM and CD45 selection. Thresholds of detection are determined from spiked samples and unspiked healthy volunteer blood. EpCAM positive, CD45 negative cells cells and matching leukocytes are each separately sorted into single wells of a 96 well skirted plate in 100ul of Gibco UltraPure distilled water in order to retain the maximum amount of CTC cells from the sort. The solution of CTC cells is then evaporated in a Thermo Scientific SpeedVac Concentrator to a volume of 4 ul, the initial volume required of the Qiagen REPLI-g Single Cell Kit. Matched germline DNA was derived from normal WBCs. The Qiagen kit uses a Multiple Displacement Amplification chemistry to create whole genome DNA sequences that generate yields of 10 ugs of DNA with an average length of greater than $10 \mathrm{~kb}$. 
The REPLI-g kit is used to amplify the DNA from our CTCs to generate enough yield to run on the aCGH copy number microarrays. After the sample is evaporated down to $4 \mathrm{ul}$, 3ul of cell lysis buffer is added to the sample and incubated at $65 \mathrm{C}$ for $10 \mathrm{~min}$. After incubation, 3 ul of Stop Solution is added to the sample reaction. The amplification mastermix is made using 9 ul of water, 29 ul of Reaction Buffer and 2 ul of DNA Polymerase for each reaction. The $10 \mathrm{ul}$ reaction is mixed with $40 \mathrm{ul}$ of the amplification mastermix and incubated in a thermocycler at 30 $\mathrm{C}$ for 8 hours, then inactivated at $65 \mathrm{C}$ for 3 mins. All reagents used in the REPLI-g kit are supplied with the kit.

The Agilent SureTag DNA Enzymatic Labeling Kit is used to label the CTC and Leukocyte DNA that is amplified by the REPLI-g kit. The Agilent labeling kit requires 0.5 ug of DNA to begin. The volume of the DNA sample is brought up to 20.2 ul with nuclease free water. A digestion master mix is made with the following components for each reaction: 2 ul of nuclease free water, 2.6 ul of 10x Restriction Enzyme Buffer, 0.2 ul of BSA, 0.5 ul of Alu I, and 0.5 ul of Rsa I. After the digestion master mix is made and mixed, $5.8 \mathrm{ul}$ of the digestion mastermix is added to the 20.2 ul of each test and reference sample. The mastermix mixed with sample reaction is incubated on a thermocycler at $37 \mathrm{C}$ for 2 hours, then at $65 \mathrm{C}$ for 20 to inactive the digestion enzymes.

In preparation for the labeling step, 5 ul random primers are added to each reaction sample. The reaction mixed with random primers is incubated at $95 \mathrm{C}$ for 3 mins on a thermocycler. The labeling master mix is made using the following compondents for each reaction: 2 ul of nuclease free water, 10 ul of $5 x$ reaction buffer, 10x dNTPs, 3 ul of Cy3 or Cy5 dUTP, and 1 ul of the Exo Klenow fragment. Mix the labeling mastermix by pipetting up and down and adding 19 ul to each reaction sample. The reaction sample with labeling master mix is incubated in a thermocycler at $37 \mathrm{C}$ for 2 hours and then inactivated at $65 \mathrm{C}$ for 10 minutes. After incubation, the reaction is ran through a filter to clean out the extraneous reagents not labeled to the DNA.

Before the hybridization is started, the labeled samples are quantitated on a nanodrop spectrophotometer for yield and quality of labeling. Labeled test and reference samples are diluted to the same yield so each can be hybridized evenly to the microarray. The test sample is transferred and mixed with the corresponding reference sample. The hybridization mastermix is composed of the following reagents per reaction: $5 \mathrm{ul}$ of Cot-1 DNA, $11 \mathrm{ul}$ of 10x aCGH Blocking Agent, and $55 \mathrm{ul}$ of HI-RPM Hybridization Buffer, for a total master mix volume of $71 \mathrm{ul}$ for each reaction. The $39 \mathrm{ul}$ volume of the labeled test/reference reaction is mixed with the $71 \mathrm{ul}$ of the hybridization master mix for a total volume of $110 \mathrm{ul}$. The test/reference hybridization reaction is then placed in a thermocycler and incubated at $95 \mathrm{C}$ for 3 minutes, then $37 \mathrm{C}$ for 30 minutes. The test/reference reaction is then aliquoted onto the algilent gaskest slide in $100 \mathrm{ul}$ amounts. The microarray slide is then laid on top of the gasket slide and the two are clamped together in the microarray chamber. The microarray chamber is then placed in a rotisserie style hybridization oven and rotated at $20 \mathrm{rpms}$ at $65 \mathrm{C}$ for 24 hours.

After the 24 hour hybridization period the microarray/gasket "sandwich" is sumberged and the microarray is separated from the gasket. Then the microarray is rinsed in different wash buffer solutions to wash away excess labeling and hybridization reagent that is not bound to the microarray. The microarray is scanned in the 4100A Genepix Scanner and the microarray data was generated in the Genepix microarray analysis software. The GC bias is corrected running a linear regression on the data generated against the percentage of GCs in the genome. The copy number calling is ran on the circular binary segmentation (CBS) algorithm. The microarray results are then uploaded into the UCSC genome browser.

Multi-image TIFF files were processed with the GenePix Analysis software using the Agilent design 014950_D.gal file created for the 4x44K microarray. The results were normalized on the ratio of medians. A GC correction was done using the known GC percentage of each probe and running a linear regression against the log ratio. The log ratio data compiled from the array is the run through the algorithms Gain and Loss Analysis of DNA (GLAD) and Circular Binary 
Segmentation (CBS) using the CGHweb online tool. The genomic build 36 that is used by the Agilent $4 \times 44 \mathrm{~K}$ microarray is converted to the genomic build 37 using the LiftOver tool from the SantaCruz genome browser. The converted build 37 microarray log ratio data files are then converted from bed files to bedgraph files as per the USCS format and uploaded into UCSC. A gain is notated as a log ratio greater than or equal to 0.2 and a loss is notated as a log ratio less than or equal to -0.2. A one copy gain is notated as a log ratio greater than or equal to 0.5 and a copy loss is notated as a log ratio less than or equal to -0.5 . Furthermore, to be called a gain or a loss the region must have at least 10 consecutive probes all above the 0.2 or below the -0.2 threshold.

We successfully profiled the genomic copy number of CTCs from 4 men with CRPC (P13, P18, P27 and P32), including several replicates and one patient with sequential CTC arrayCGH analyses in the context of enzalutamide therapy. Our copy number profiling is displayed in the UCSC genome browser as a bedgraph file. Each sample hybridized with its own leukocytes as a reference. Each graphical representation of the copy number profile or track depicts a copy number gain, shown in green, or a copy number loss, shown in red. A log ratio greater than 0.2 or less than -0.2 represents a copy number gain or loss, respectively. Metastatic reference is included to compare with. Patient 18.2 is a man with mCRPC who progressed on abiraterone and did not respond to enzalutamide. Subject 27 is another man with mCRPC who did respond to enzalutamide initially (27.1), but quickly developed resistance to enzalutamide in about $5 \mathrm{~m}$ (27.3). Patient 13.3 is a man with mCRPC who did not respond to enzalutamide. Similar to P27, patient 32 progressed on enzalutamide quickly after initial brief response and was then put on study drug. He responded to the study drug (32.1) initially, but developed resistance soon (32.3). Figure 4 summarized the clinical course of these 4 patients.
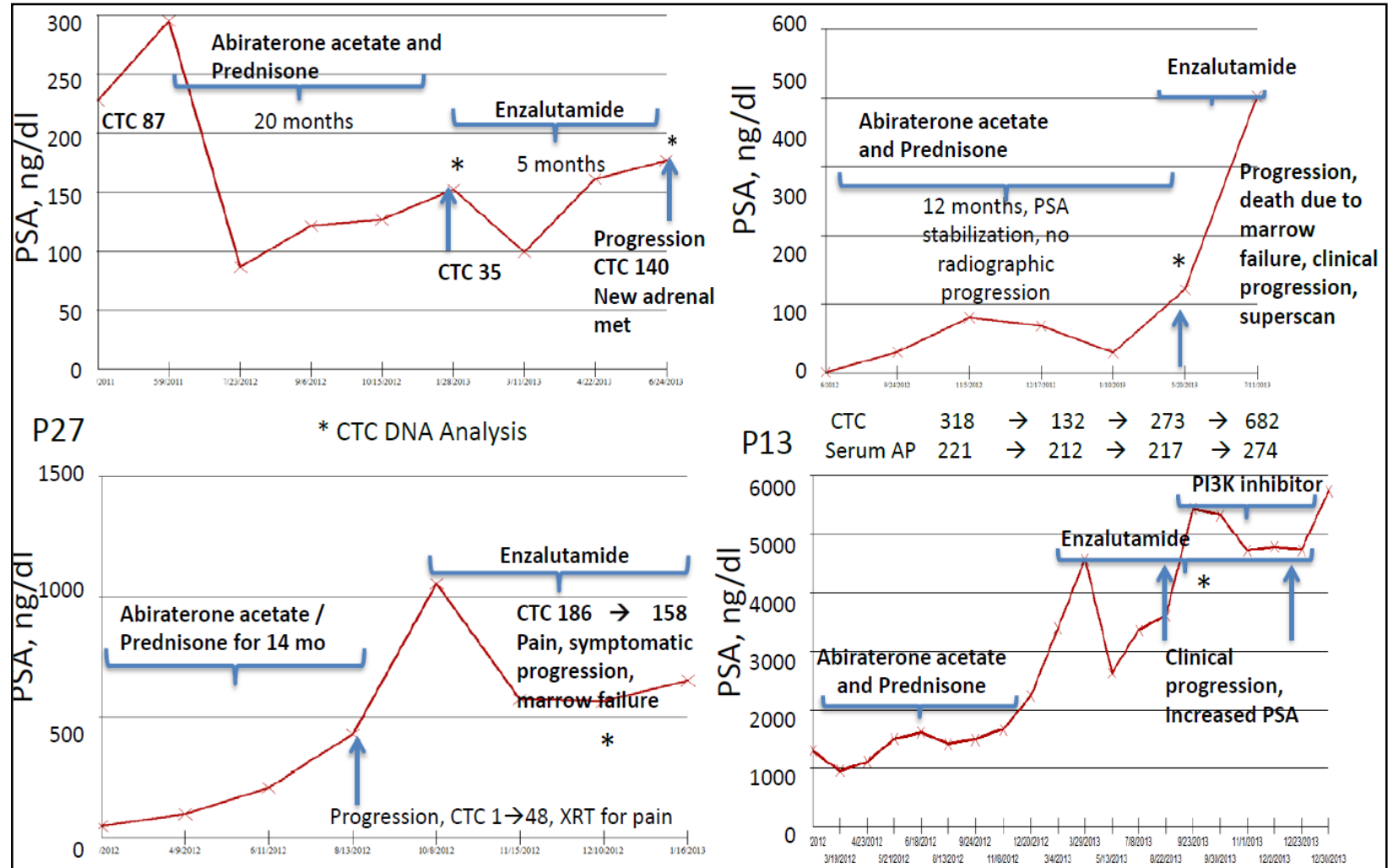

P18

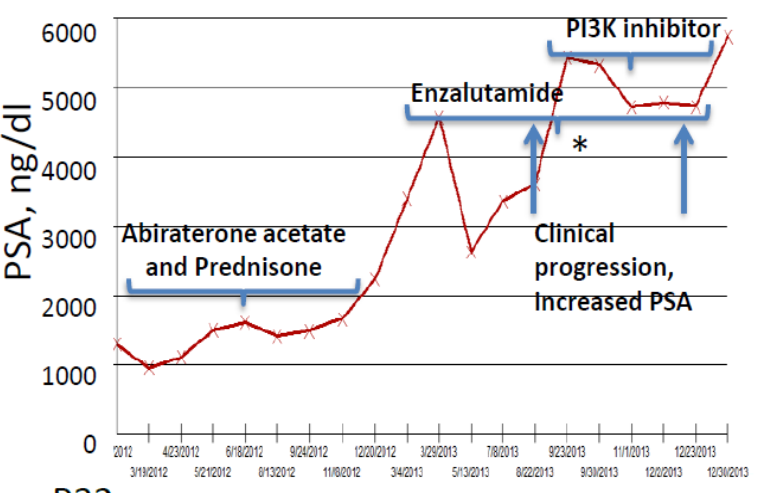
P32

Figure 4. Clinical course of 4 men with mCRPC treated with enzalutamide after abiraterone acetate. * represents the time point of the CTC molecular analyses. 
Patient 27 is a man with mCRPC who responded to enzalutamide briefly after abiraterone acetate but developed visceral progression (adrenal, CNS). His CTCs collected prior to starting enzalutamide (P27.1) revealed AR amplification. He then developed resistance to enzalutamide (P27.3), when CTCs were re-collected and interestingly revealed loss of AR gene locus amplification. On the contrary, patient 18 who had de novo resistance to enzalutamide had loss of the AR copy numbers in his CTCs collected while he was on enzalutamide. Patient 13 is another man with de novo enzalutamide resistant CRPC, whose AR is wild type. Patient 32 is a man with CRPC, who responded to a study drug briefly and then developed resistance. His CTCs collected prior to starting the study drug (P32.1) revealed AR wild type. After he developed resistance (P32.3), CTCs were re-collected and aCGH analysis for P32.3 is pending for now (Figure 2). The results indicate that AR amplification can be potentially a predictive biomarker of response to novel ADT.

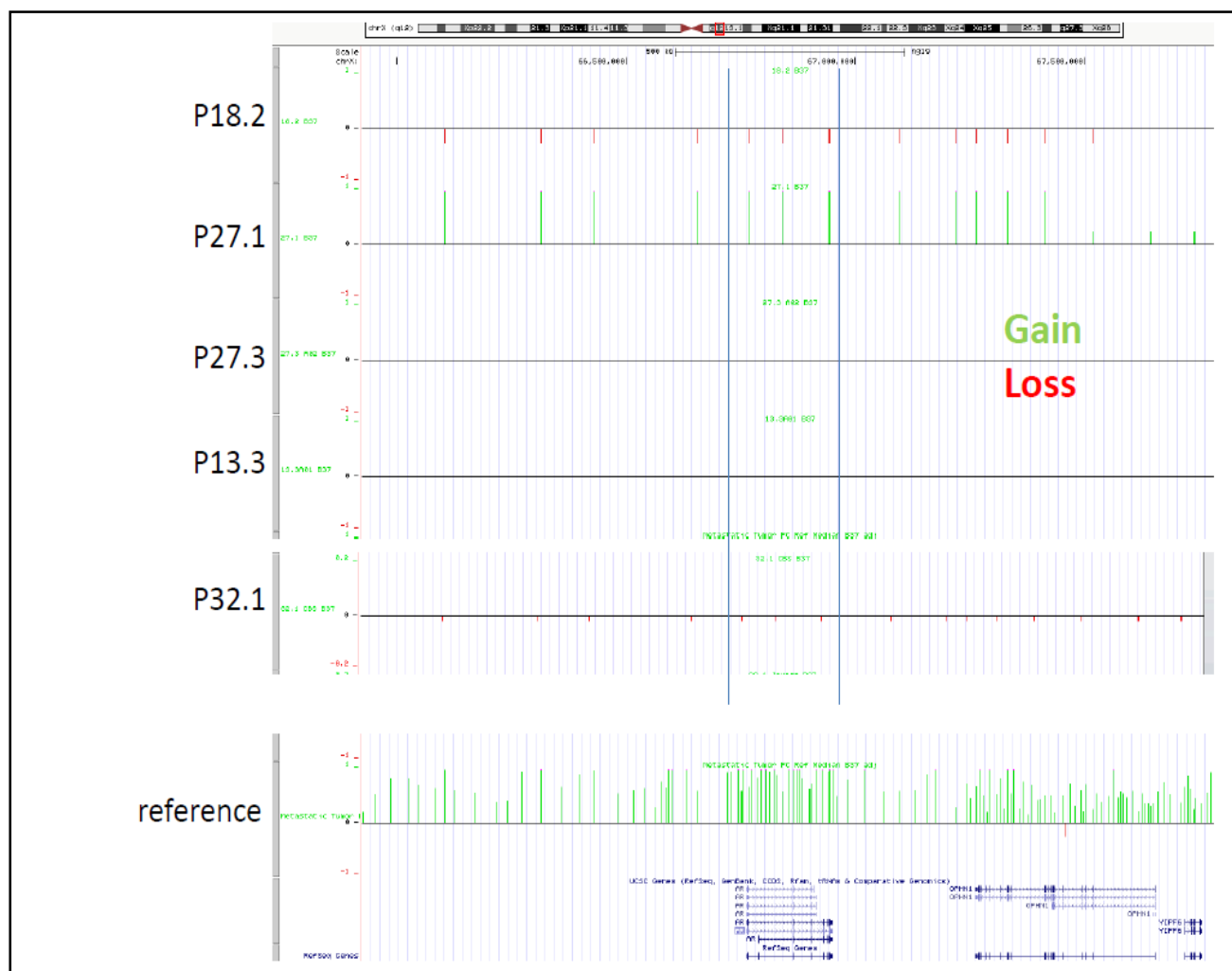

Figure 5. Analysis of the genomic locus of AR (X-chromosome) by array CGH in our patients with mCRPC. Gain or amplication is represented in green as above the x-axis (at least 0.2 copy number on the log scale) while loss or deletion is indicated in red (at least -0.2 on the log scale). For P27 for example, his pre-enzalutamide AR status was gain (P27.1) while his post-enzalutamide AR status showed loss of this gain (wild type). Reference mCRPC DNA from Taylor et al (MSKCC data) is provided on the bottom row.

In patient 27, MYCN copy number loss was demonstrated in CTCs collected prior to starting enzalutamide (27.1). In the contrary, MYCN gene locus is amplified in CTCs from the same patient collected after he developed enzalutamide resistance (27.3) (Figure 3). MYCN amplification is seen in $40 \%$ of NEPC and $5 \%$ of PCA, respectively, and is found to induce a neuroendocrine phenotype in prostate cells $\mathbf{s}^{5}$. MYCN amplifications may contribute to treatment induced NEPC after hormonal therapy and lead to resistance to ADT. MYCN copy number changes were not seen in the other patients. These data suggest that in this patient, emergence of tumor clones that had loss of AR amplification and gain of the proliferative gene MYCN corresponded to clinical progression on enzalutamide and visceral spread of his disease. 


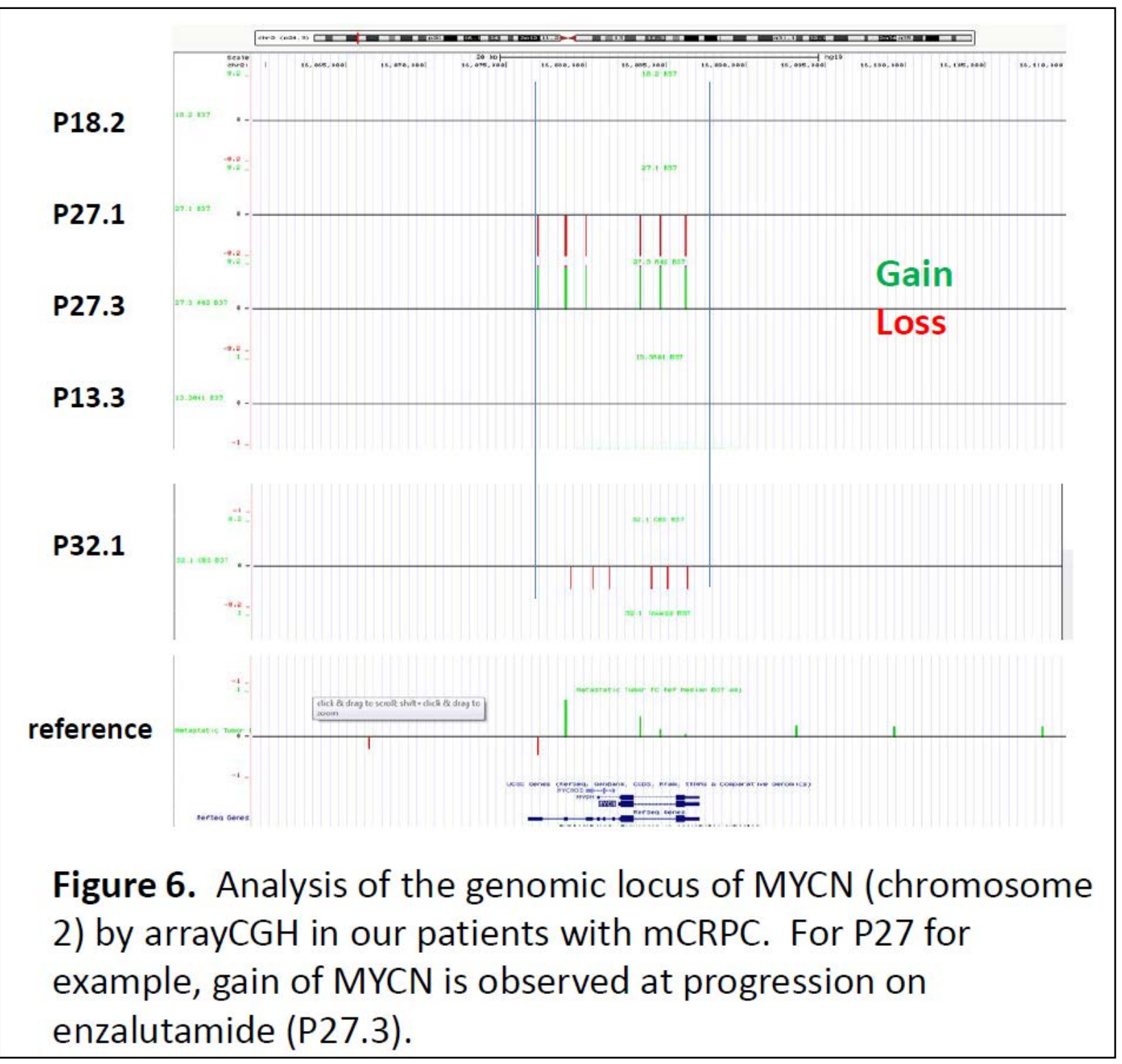


Enhanced $A B L$ expression and activation were reported in some solid tumors (breast cancer, uterine endometrioid cancer, ovarian cancer, NSCLC). We found ABL1 amplification in two patients in P18.2 and P13.3 (Figure 7). ABL2 amplification was discovered in P27.1. The physiologic role of function of $A B L 1$ and $A B L 2$ in prostate cancer needs further evaluation but $A B L$ kinases have been implicated in bone metastases in many solid tumors ${ }^{6}$. The results indicate that ABL1/2 may be implicated in CRPC bone metastatic progression and a potential target for therapy; however, further study on the function of $A B L 1 / 2$ gain in this disease is needed.

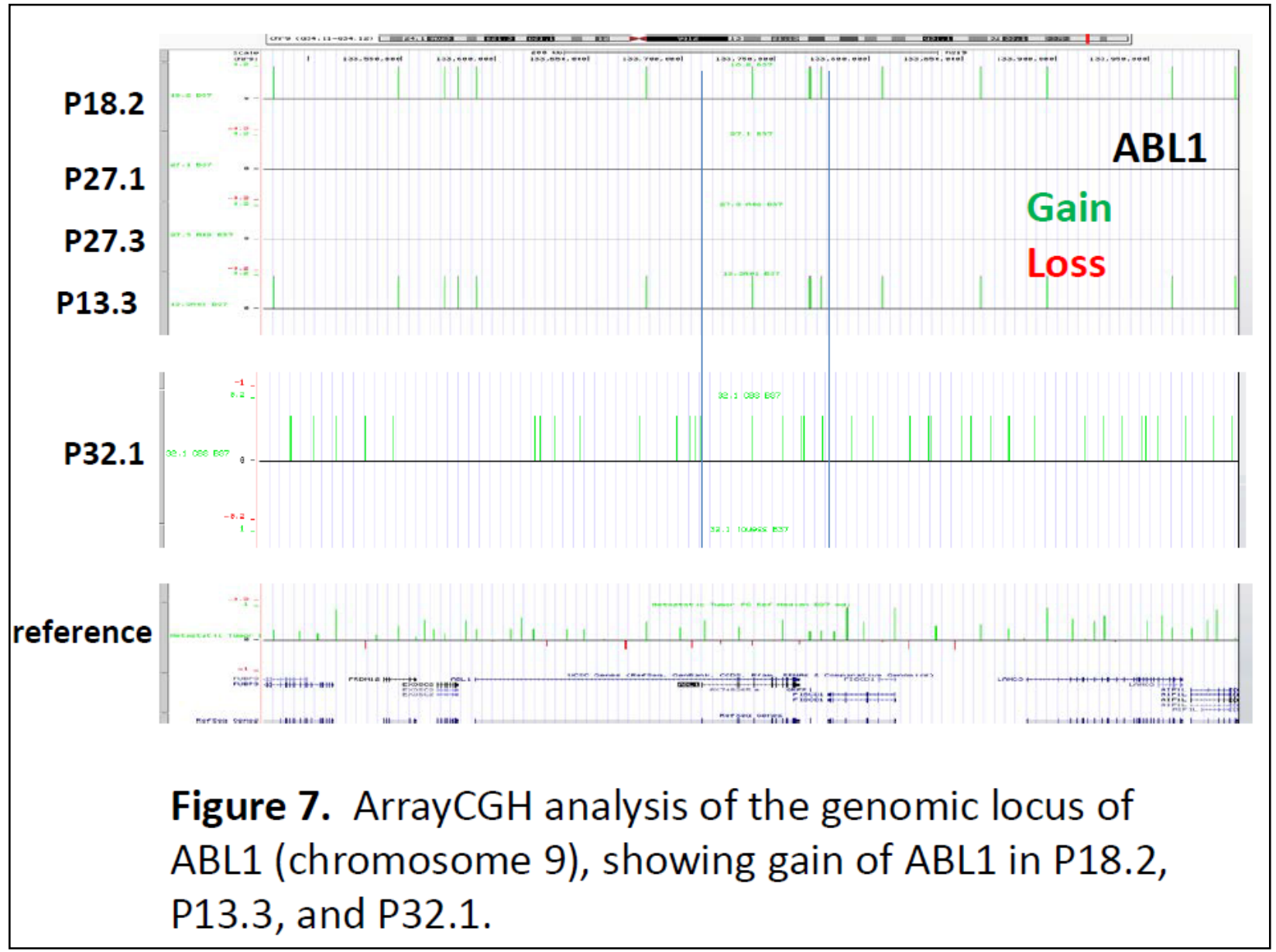

We compiled a list of all copy gains and losses observed by arrayCGH in our 4 men with $\mathrm{mCRPC}$, with a particular focus on those genes highly implicated in CRPC progression and previously validated as being aberrant in metastatic tissue samples and genomic studies of reference datasets. As can be seen in table 1, a number of copy gains in AR, EZH2, SPOP, CCND1, BRD4, MYC, FOXA1, and other genes were observed, while common losses of the locus including CHD1, c-MET, FGFR2, PHLLP, NCOR1, NCOR2 were seen. These data imply a shift away from AR signaling toward additional oncogenic programs in these men, and a highly complex molecular profile that includes aberrations in androgen signaling, oncogenic pathways including c-MET, PI3K, RB1, p53, ABL kinases, and MYC/MYCN signaling, as well as cell cycle and epigenetic pathways during CRPC progression. Further collection of genomic data during sequential follow up of men with mCRPC during treatment with novel hormonal therapies such as enzalutamide or abiraterone acetate to detect common and acquired genomic changes that are associated with progression and resistance will be essential. Our data confirms the feasibility of a CTC-based genomic assay for copy number alternations. Data using dye swaps and repeat samples confirms the validity of each of these cases (P27, P18, P13) and copy alternations, and further studies of repeat measures will be needed to ensure that this platform can be useful as a biomarker for multicenter correlative studies of CRPC biology. 


\begin{tabular}{|c|c|c|c|c|c|c|c|}
\hline Function & $\begin{array}{l}\text { Genomic } \\
\text { lesions }\end{array}$ & Reference & $\begin{array}{l}\text { P27 } \\
\text { pre- } \\
\text { enza }\end{array}$ & $\begin{array}{l}\text { P27 } \\
\text { post- } \\
\text { enza }\end{array}$ & P18.2 & P13.3 & P32.1 \\
\hline \multirow[t]{11}{*}{ Androgen } & AR & & & & & & \\
\hline & FOXA1 & & & & & & \\
\hline & NRAS & & & & & & \\
\hline & CYP11B1 & & & & & & \\
\hline & NKX3-1 & & & & & & \\
\hline & GATA2 & & & & & & \\
\hline & HSD17B4 & & & & & & \\
\hline & PXN & & & & & & \\
\hline & CYP11A1 & & & & & & \\
\hline & UGT2B17 & & & & & & \\
\hline & PIAS3 & & & & & & \\
\hline \multirow[t]{8}{*}{ Oncogene } & MYC & & & & & & \\
\hline & MYCN & & & & & & \\
\hline & c-MET & & & & & & \\
\hline & FGFR2 & & & & & & \\
\hline & SPOP & & & & & & \\
\hline & ABL1 & & & & & & \\
\hline & $\overline{\text { ABL2 }}$ & & & & & & \\
\hline & BRAF & & & & & & \\
\hline \multirow[t]{6}{*}{ Tumor suppressor } & PTEN & & & & & & \\
\hline & PHLPP1 & & & & & & \\
\hline & $\mathrm{RB}$ & & & & & & \\
\hline & RAF1 & & & & & & \\
\hline & P53 & & & & & & \\
\hline & APC & & & & & & \\
\hline \multirow[t]{3}{*}{ Cell cycle } & AURKA & & & & & & \\
\hline & CCND1 & & & & & & \\
\hline & CCNE1 & & & & & & \\
\hline \multirow{8}{*}{$\begin{array}{l}\text { Histone/chromatin } \\
\text { modifiers }\end{array}$} & EZH2 & & & & & & \\
\hline & NCOR1 & & & & & & \\
\hline & NCOR2 & & & & & & \\
\hline & CHD1 & & & & & & \\
\hline & CDK12 & & & & & & \\
\hline & MLL3/KMT2C & & & & & & \\
\hline & MLL2/KMT2D & & & & & & \\
\hline & UTX/KDM6A & & & & & & \\
\hline
\end{tabular}




\begin{tabular}{|l|l|l|l|l|l|l|l|}
\hline & MYST4/KAT6B & & & & & & \\
\hline \multirow{4}{*}{$\begin{array}{l}\text { Transcriptional } \\
\text { factor }\end{array}$} & BRD4 & & & & & & \\
\hline & ZFHX3 & & & & & \\
\hline & NCOA2 & & & & & & \\
\hline \multirow{3}{*}{ Misc } & TRAP1 & & & & & & \\
\cline { 2 - 8 } & PDE4DIP & & & & & & \\
\hline & AKAP9 & & & & & & \\
\hline
\end{tabular}

Table 1. Summary of arrayCGH results in CTCs from 4 men with mCRPC. Red indicates copy gain, blue indicates copy loss, and white represents lack of gain or loss (wild type).

Whole exome sequencing (WES) on REPLI-g amplified DNA from CTC and leukocyte was performed using GeneWiz and the TruSeq Exome Capture Kit, and was then sequenced on the Illumina HiSeq 2000 with 40x coverage. In brief, by using an E210 ultrasonicator, the genomic DNA samples were randomly fragmented into 250-300 bp fragments. Fragmented DNA was subjected to library preparation. Exome capture was performed using the Agilent v2 Human Exon bait kit. Captured DNA was sequenced using the Illumina HiSeq platform. Initial alignment and quality control were performed using the Picard and Firehose pipelines at the Broad Institute. Picard generates a single BAM file for each sample that includes reads, calibrated quantities and alignments to the genome. Firehose is a set of tools for analyzing sequencing data from tumor and matched normal DNA. The pipeline uses GenePattern as its execution engine and performs quality control, local realignment, mutation calling and coverage calculations, among other analyses. Sequencing was performed to average target coverage of more than 40x. Reads were aligned to the reference human genome build hg19 through implementation of the Burrows-Wheeler Aligner and processed through Picard. Single nucleotide variant (SNV) or insertion/deletion (INDEL) was called if the site is covered by more than 10 reads.

The resulting FASTQ sequences underwent a paired-end aligned to the NCBI human genome assembly (build 37, hg19) using the Burrows-Wheeler Aligner (BWA) program version 0.7.9a for all exome sequencing data. The sequences were trimmed and edited using the programs samtools version 0.1.19. PCR duplicates were marked and removed using Picard. The sequence variants were called using Genome Analysis Tool Kit (GATK) version 3.1. A mutation was called if no mutant reads for an allele were observed in germline DNA at a locus that was covered at least 10 fold, and if at least 5 reads supporting the mutant were found in the CTC exome data with at least 1 read on each strand (forward and reverse) and also covered at least 10 fold. 
We successfully sequenced exome from CTCs and leukocytes from patient 18.2, who progressed through enzalutamide therapy. We discovered many acquired mutations in CTCs exome, including SNV and INDEL (Figure 8). In summary, we identified totally 138 SNV including 100 heterozygous mutations and 38 homozygous mutations in CTCs; and 47 INDEL aberrations in CTCs, with 22 heterozygous changes and 25 homozygous changes. Part of the exome sequencing aberrations (89 out of 185) occurred in genes, which have been reported in prostate cancer by COSMIC search. We went though all mutated genes in pubmed and 9 genes with SNV aberration (Table 2) and 7 genes with INDEL changes (Table 3) were reported to be implicated in prostate cancer or cancer metastasis.

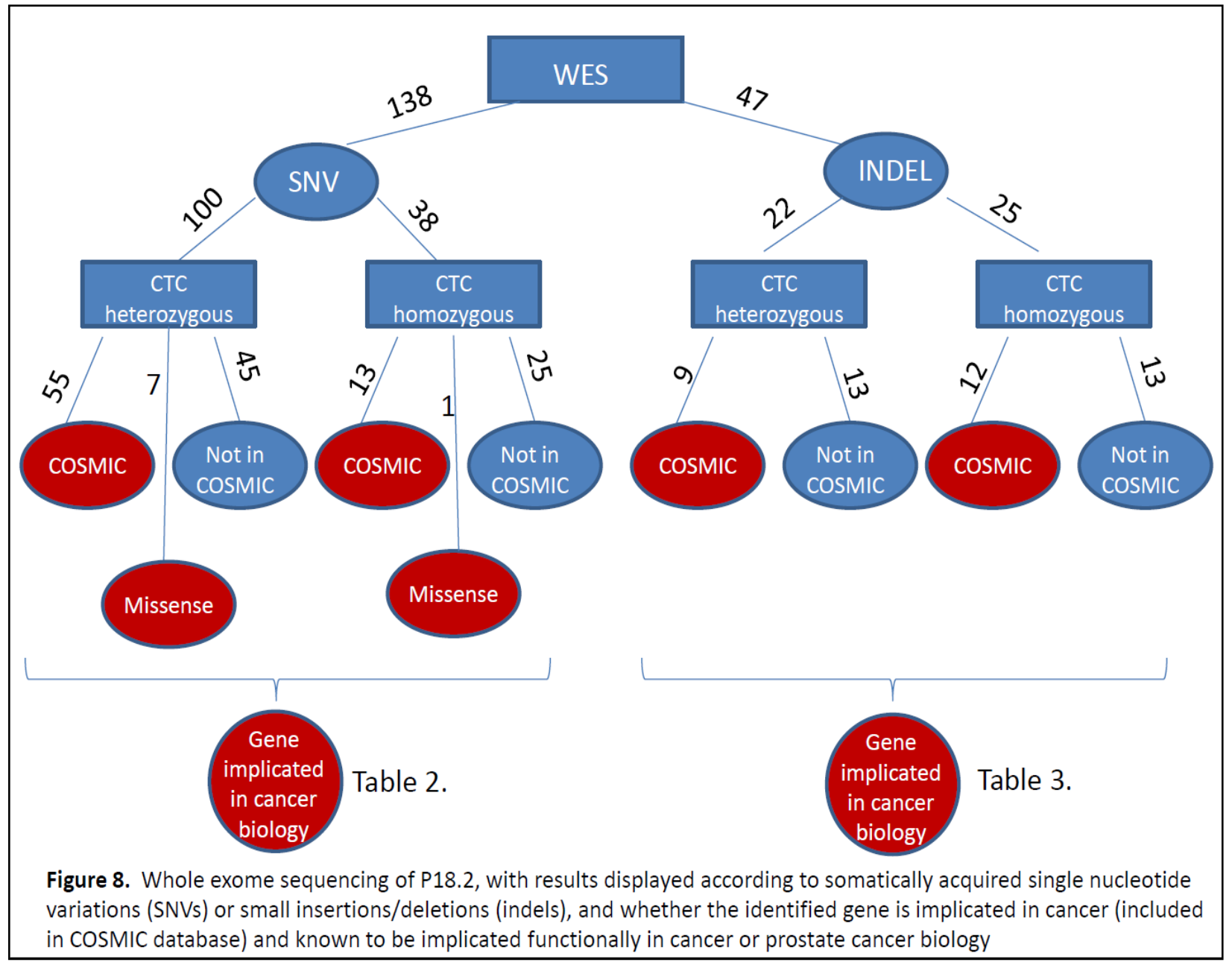




\begin{tabular}{|c|c|c|c|c|}
\hline Gene & Position & Gene name & Reported function & Pubmed Ref \\
\hline PIF1 & chr15:65114833 & PIF1 5'-To-3' DNA Helicase & $\begin{array}{l}\text { protect human tumor cells } \\
\text { from apoptosis }\end{array}$ & $\begin{array}{l}\text { Cancer Res. } 2011 \text { Jul } \\
\text { 15;71(14):4998-5008 }\end{array}$ \\
\hline MAX & chr14:65550816 & MYC Associated Factor X & $\begin{array}{l}\text { Pairs with MYC to regulation } \\
\text { proliferation, androgenic } \\
\text { control of AR mRNA }\end{array}$ & $\begin{array}{l}\text { Mol Endocrinol. } 1999 \\
\text { Nov;13(11):1896-911. }\end{array}$ \\
\hline NOD1 & chr7:30498842 & $\begin{array}{c}\text { Nucleotide-Binding } \\
\text { Oligomerization Domain } \\
\text { Containing } 1\end{array}$ & $\begin{array}{l}\text { development and } \\
\text { progression of prostate } \\
\text { cancer }\end{array}$ & $\begin{array}{l}\text { Prostate. } 2012 \text { Sep } \\
\text { 1;72(12):1351-8 }\end{array}$ \\
\hline NCOA1 & $\operatorname{chr} 2: 24962252$ & $\begin{array}{l}\text { Nuclear Receptor } \\
\text { Coactivator } 1\end{array}$ & AR coregulator & $\begin{array}{l}\text { Endocrine Reviews } \\
23(2): 175-200\end{array}$ \\
\hline SEMA3A & chr7:83739762 & $\begin{array}{l}\text { Sema Domain, } \\
\text { Immunoglobulin Domain } \\
\text { (Ig), Short Basic Domain, } \\
\text { Secreted, } \\
\text { (Semaphorin) 3A }\end{array}$ & $\begin{array}{c}\text { Predictive value of } \\
\text { biochemical in low-and } \\
\text { intermediate-risk prostate } \\
\text { cancer }\end{array}$ & $\begin{array}{l}\text { Neoplasma. 2013;60(6):683- } \\
9 .\end{array}$ \\
\hline USP10 & chr16:84808900 & $\begin{array}{l}\text { Ubiquitin Specific Peptidase } \\
10\end{array}$ & $\begin{array}{l}\text { required for androgen } \\
\text { receptor-mediated gene } \\
\text { activation }\end{array}$ & $\begin{array}{l}\text { Nucleic Acids Res. } 2011 \\
\text { May;39(9):3529-42 }\end{array}$ \\
\hline VDAC1 & chr5:133328560 & $\begin{array}{l}\text { Voltage-Dependent Anion } \\
\text { Channel } 1\end{array}$ & $\begin{array}{l}\text { Cancer Cell Proliferation and } \\
\text { Tumor Growth In Vivo }\end{array}$ & $\begin{array}{c}\text { Mol Ther Nucleic Acids. } 2014 \\
\text { Apr 29;3:e159 }\end{array}$ \\
\hline $\mathrm{CDON}$ & chr11:125888213 & $\begin{array}{l}\text { Cell Adhesion Associated, } \\
\text { Oncogene Regulated }\end{array}$ & $\begin{array}{l}\text { tumor cell growth and } \\
\text { invasion }\end{array}$ & $\begin{array}{c}\text { Pathobiology. } \\
\text { 2011;78(5):277-84 }\end{array}$ \\
\hline PTGER3 & chr1:71471875 & Prostaglandin E Receptor 3 & $\begin{array}{l}\text { castration resistance in } \\
\text { prostate cancer cells }\end{array}$ & $\begin{array}{l}\text { Endocr Relat Cancer. } 2013 \\
\text { May 30;20(3):431-41 }\end{array}$ \\
\hline
\end{tabular}

Table 2. Selected genes with single nucleotide polymorphisms somatically acquired in P18.2's circulating tumor cells and not present in paired leukocytes. These genes have been implicated in prostate cancer metastatic biology or cancer metastasis. These SNVs are synonymous mutations or found in the untranslated regions of the specified gene.

\begin{tabular}{|c|c|c|c|c|}
\hline Gene & Position & Gene name & Reported function & Pubmed Ref \\
\hline PECAM 1 & chr17:62400086 & $\begin{array}{l}\text { Platelet/Endothelial Cell } \\
\text { Adhesion Molecule } 1\end{array}$ & $\begin{array}{l}\text { promotes tumor cell } \\
\text { anchorage-independent } \\
\text { growth }\end{array}$ & Molecular Cancer 2010, 9:7 \\
\hline ITGA2 & chr5:52387187 & $\begin{array}{l}\text { Integrin, Alpha } 2 \text { (CD49B, } \\
\text { Alpha } 2 \text { Subunit Of VLA-2 } \\
\text { Receptor) }\end{array}$ & $\begin{array}{l}\text { may play roles as tumor } \\
\text { suppressor genes or } \\
\text { oncogenes }\end{array}$ & $\begin{array}{l}\text { Int J Mol Med. } 2011 \\
\text { Oct; } 28(4): 605-11\end{array}$ \\
\hline TES & chr7:115897692 & Testis Derived Transcript & $\begin{array}{l}\text { may be associated with } \\
\text { prostate cancer in black men }\end{array}$ & $\begin{array}{l}\text { J Urol. } 2007 \mathrm{Mar} ; 177(3): 894- \\
8\end{array}$ \\
\hline COL4A1 & chr13:110861647 & Collagen, Type IV, Alpha 1 & $\begin{array}{l}\text { major antiangiogenicgene } \\
\text { induced by p53 in human } \\
\text { adenocarinoma cells }\end{array}$ & 012 Mar 1;72(5):1270-9 \\
\hline HOXC8 & $\operatorname{chr} 12: 54406124$ & Homeobox $\mathrm{C} 8$ & $\begin{array}{c}\text { inhibiting SRC-3 recruitment } \\
\text { to direct androgen target } \\
\text { genes }\end{array}$ & $\begin{array}{l}\text { Mol Cancer Res. } 2010 \\
\text { Dec;8(12):1643-55 }\end{array}$ \\
\hline MMP8 & chr11:102584466 & $\begin{array}{l}\text { Matrix Metallopeptidase } 8 \\
\text { (Neutrophil Collagenase) }\end{array}$ & $\begin{array}{l}\text { Metastasis Suppressor } \\
\text { through Modulation of } \\
\text { Tumor Cell Adhesion and } \\
\text { Invasion }\end{array}$ & $\begin{array}{l}\text { Cancer Res April 15, } 2008 \\
\text { 68; } 2755\end{array}$ \\
\hline MLL & chr11:118344185 & $\begin{array}{l}\text { Histone-lysine } \mathrm{N} \text { - } \\
\text { methyltransferase HRX }\end{array}$ & $\begin{array}{l}\text { MLL2, mutated in } 8.6 \% \text { of } \\
\text { prostate cancers. }\end{array}$ & $\begin{array}{l}\text { Nature } 487,239-243 \text { (12 } \\
\text { July 2012) }\end{array}$ \\
\hline
\end{tabular}

Table 3. Selected genes with small insertions and deletions (INDELS) somatically acquired in P18.2's circulating tumor cells and not present in paired leukocytes. These genes have been implicated in prostate cancer metastatic biology or cancer metastasis. 
We searched coding sequence location and consequence of our identified SNV and INDEL aberrations by using ENSEMBL website. Among all the 185 genomic aberrations, only 8 mutations led to missense and likely non-synonymous changes, with all the others are either synonymous changes or being in intron, UTR, or non coding extron (Table 4). These genes may be important in cancer biology but may also be silent or private mutations of unclear significance and further evaluation of these genes across a larger data set is needed.

\begin{tabular}{|c|c|c|c|c|c|}
\hline Location & ID & CDS position & Gene & Gene name & Gene Function \\
\hline 8:133909974 & rs853326 & 3082 & TG & thyroglobulin & $\begin{array}{l}\text { Precursor of the } \\
\text { iodinated thyroid } \\
\text { hormones thyroxine (T4) } \\
\text { and triiodothyronine } \\
\text { (T3) }\end{array}$ \\
\hline $10: 124096061$ & rs1048347 & 1316 & BTBD16 & $\begin{array}{l}\text { BTB (POZ) Domain } \\
\text { Containing } 16\end{array}$ & $\begin{array}{l}\text { Associated with } \\
\text { Alzheimer disease }\end{array}$ \\
\hline $2: 160086654$ & rs55738533 & 4717 & TANC1 & $\begin{array}{l}\text { tetratricopeptide } \\
\text { repeat, ankyrin repeat } \\
\text { and coiled-coil } \\
\text { containing } 1\end{array}$ & $\begin{array}{l}\text { May be a scaffold } \\
\text { component in the } \\
\text { postsynaptic density }\end{array}$ \\
\hline $2: 17962518$ & rs300169 & 2039 & GEN1/SMC6 & $\begin{array}{l}\text { GEN1 Holliday Junction } \\
\text { 5' Flap Endonuclease }\end{array}$ & $\begin{array}{l}\text { Endonuclease which } \\
\text { resolves Holliday junctions }\end{array}$ \\
\hline $20: 62293272$ & rs3848668 & 371 & RTEL1/RTEL1-TNFRSF6B & $\begin{array}{l}\text { RTEL1-TNFRSF6B } \\
\text { Readthrough }\end{array}$ & $\begin{array}{l}\text { candidate for nonsense- } \\
\text { mediated mRNA decay } \\
\text { (NMD), and is unlikely to } \\
\text { produce a protein } \\
\text { product }\end{array}$ \\
\hline $19: 50251422$ & rs34701020 & 499 & TSKS & $\begin{array}{l}\text { Testis-Specific Serine } \\
\text { Kinase Substrate }\end{array}$ & $\begin{array}{c}\text { May play a role in testicular } \\
\text { physiology, most probably } \\
\text { in the process of } \\
\text { spermatogenesis or } \\
\text { spermatid } \\
\text { development }\end{array}$ \\
\hline $19: 40030704$ & rs7252027 & 16 & EID2 & $\begin{array}{l}\text { EP300 Interacting } \\
\text { Inhibitor of } \\
\text { Differentiation } 2\end{array}$ & $\begin{array}{l}\text { Interacts with EP300 and } \\
\text { acts as a repressor of } \\
\text { MYOD-dependent } \\
\text { transcription and muscle } \\
\text { differentiation. }\end{array}$ \\
\hline $2: 141115595$ & rs148734150 & 11348 & LRP1B & $\begin{array}{c}\text { Low Density Lipoprotein } \\
\text { Receptor-Related } \\
\text { Protein 1B }\end{array}$ & $\begin{array}{l}\text { Potential cell surface } \\
\text { proteins that bind and } \\
\text { internalize ligands in the } \\
\text { process of receptor- } \\
\text { mediated } \\
\text { endocytosis }\end{array}$ \\
\hline
\end{tabular}

Table 4. Selected genes with SNVs resulting in missense alterations and nonsynonymous changes in protein structure. The genes listed in this table are not necessarily implicated in cancer or prostate cancer progression. 
There are 12 novel INDELs have never been reported by any SNP database (Table 5). Notable among these are FAN1, implicated in DNA repair, and MLL, implicated in epigenetic regulation of the genome and prostate cancer progression.

\begin{tabular}{|c|c|c|c|}
\hline Gene & Gene Name & Location & Changes \\
\hline DGKH & Diacylglycerol Kinase & $13: 42803722$ & $\mathrm{C}-\mathrm{CG}$ \\
\hline TLN2 & Talin 2 & $15: 63134165$ & CCT-C \\
\hline ETFDH & $\begin{array}{l}\text { Electron-Transferring- } \\
\text { Flavoprotein Dehydrogenase }\end{array}$ & 4:159618695 & CT-C \\
\hline FAN1 & $\begin{array}{l}\text { FANCD2/FANCI-Associated } \\
\text { Nuclease } 1\end{array}$ & $15: 31231754$ & TTTGA-T \\
\hline XRRA1 & $\begin{array}{l}\text { X-Ray Radiation Resistance } \\
\text { Associated } 1\end{array}$ & $11: 74641436$ & GA-G \\
\hline LRRN4CL & $\begin{array}{l}\text { LRRN4 C-Terminal-Like } \\
\text { Protein }\end{array}$ & $11: 62453942$ & A-AG \\
\hline NSL1 & MIS12 Kinetochore Complex & $1: 212900434$ & C-CG \\
\hline LRP1B & $\begin{array}{l}\text { Low Density Lipoprotein } \\
\text { Receptor-Related Protein 1B }\end{array}$ & $2: 141135863$ & T-TA \\
\hline POLE3 & $\begin{array}{l}\text { Polymerase (DNA Directed), } \\
\text { Epsilon 3, Accessory Subunit }\end{array}$ & $9: 116172488$ & G-GC \\
\hline $\mathrm{HOXC} 8$ & Homeobox C8 & $12: 54406124$ & $\mathrm{CA}-\mathrm{C}$ \\
\hline MLL & $\begin{array}{l}\text { Histone-lysine N- } \\
\text { methyltransferase HRX }\end{array}$ & $11: 118344185$ & $\mathrm{~A}-\mathrm{AC}$ \\
\hline ANKRD12 & Ankyrin Repeat Domain 12 & 18:9279688 & T-TA \\
\hline
\end{tabular}

Table 5. Novel INDELS identified in patient P18.2. 
Genes in table 2 and table 3 were clustered by ConsensusPathDB with enriched pathway based sets and results are summarized in Figure 9. These results demonstrate the potential for the proof of concept of the main aim of this project to identify genes in CTCs of men with mCRPC that may be implicated in prostate cancer metastasis and progression. Common pathways in P18.2 associated with SNVs and indels included developmental biology and stemness, invasion/migration, neuroendocrine transformation and small cell cancer, and neuronal migration/axon guidance. These data imply a stem-like neuroendocrine genotype/phenotype in this patient who did not respond to enzalutamide. With the success of whole exome sequencing in one patient's sample, we plan to proceed to sequence CTC exomes additional men with mCRPC as part of the PCF-Movember Global Treatment Sciences Challenge Award. Identification of common sequencing aberration events among the MCRPC patients in the context of hormonal and taxane resistance may lead to the identification of predictive biomarkers of specific therapies and potential targets for novel therapies or combinations.

\begin{tabular}{|c|c|c|c|c|c|c|}
\hline \multicolumn{7}{|c|}{ 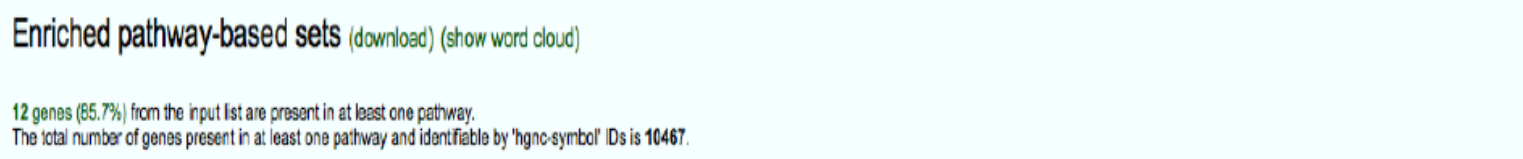 } \\
\hline $\begin{array}{l}\text { select } \\
\text { all none }\end{array}$ & pathway name & set size & $\begin{array}{l}\text { candidates } \\
\text { contained }\end{array}$ & p-value & q-value & pathway source \\
\hline 0 & Developmental Biology & 351 & $5(1.4 \%)$ & $269 \mathrm{e}-05$ & 0.000481 & Reactome \\
\hline 0 & Integrin cell surface interactions & 66 & $3(4.5 \%)$ & $5.06 \mathrm{e}-05$ & 0.000481 & Reactome \\
\hline 0 & Small cell lung cancer - Homo sepiens (human) & 86 & $3(3.5 \%)$ & 0.000112 & 0.000707 & KEGG \\
\hline 0 & Extracellular matixix organization & 261 & $4(1.5 \%)$ & 0.00016 & 0.000759 & Reactome \\
\hline 0 & Beta3 integrin cell surace interactions & 44 & $2(4.5 \%)$ & 0.00111 & 0.00422 & PID \\
\hline 0 & Platelet Aggregation Inhibitor Pathway, Pharmacodynamics & 49 & $2(4.1 \%)$ & 0.00138 & 0.00435 & PharmGKB \\
\hline 0 & Integrin cell suraces interactions & 55 & $2(3.6 \%)$ & 0.00173 & 0.0047 & PID \\
\hline 0 & Beta1 iniegrin cell surace interactions & 66 & $2(3.0 \%)$ & 0.00248 & 0.00577 & PID \\
\hline 0 & Axon guidance & 257 & $3(1.2 \%)$ & 0.00273 & 0.00577 & Reactome \\
\hline 0 & Regulation of nudear SMAD2/3 signaling & 77 & $2(2.6 \%)$ & 0.00336 & 0.00639 & PID \\
\hline 0 & ECM-recepitor interaction - Homo sapiens (human) & 86 & $2(2.3 \%)$ & 0.00417 & 0.00721 & KEGG \\
\hline 0 & Pathways in cancer - Homo sapiens (human) & 327 & $3(0.9 \%)$ & 0.00539 & 0.00853 & KEGG \\
\hline 0 & Integrin & 124 & $2(1.6 \%)$ & 0.0085 & 0.0124 & INOH \\
\hline $\begin{array}{l}\text { Fi } \\
\text { in } \\
\text { ea } \\
\text { dis }\end{array}$ & $\begin{array}{l}\text { ure } 9 . \text { Pathway summ } \\
\text { cating pathways by C } \\
\text { h pathway dataset in } \\
\text { overy rate (q-value) i }\end{array}$ & 8.2 & $\begin{array}{l}\text { ased } \\
\text { B. Tr } \\
\text { isted }\end{array}$ & $\begin{array}{l}\text { Wu } \\
\text { ned }\end{array}$ & $\begin{array}{l}\text { dat } \\
\text { ber } \\
\text { vith }\end{array}$ & genes ir \\
\hline
\end{tabular}




\begin{tabular}{|c|c|}
\hline Pathway Name & Candidates contained \\
\hline Developmental Biology & COL4A1, CDON, NCOA1, ITGA2, SEMA3A \\
\hline Integrin cell surface interactions & ITGA2, COL4A1, PECAM1 \\
\hline Small cell lung cancer & MAX , ITGA2, COL4A1 \\
\hline Extracellular matrix organization & PECAM1, COL4A1, MMP8, ITGA2 \\
\hline Beta3 integrin cell surface interactions & COL4A1, PECAM1 \\
\hline $\begin{array}{l}\text { Platelet Aggregation Inhibitor } \\
\text { Pathway, Pharmacodynamics }\end{array}$ & COL4A1, ITGA2 \\
\hline Integrin cell surface interactions & ITGA2, PECAM1 \\
\hline Beta1 integrin cell surface interactions & COL4A1, ITGA2 \\
\hline Regulation of Telomerase & PIF1, MAX \\
\hline Axon guidance & SEMA3A, ITGA2, COL4A1 \\
\hline $\begin{array}{r}\text { Regulation of nuclear SMAD2/3 } \\
\text { signaling }\end{array}$ & MAX , NCOA1 \\
\hline ECM-receptor interaction & ITGA2, COL4A1 \\
\hline Pathways in cancer & ITGA2, COL4A1, MAX \\
\hline Integrin signaling & COL4A1, ITGA2 \\
\hline
\end{tabular}

Table 6. Pathways in P18.2 identified by WES by ConsensusPathDB including candidate genes with SNVs or INDELs in the selected pathways.

These results indicate the feasibility of arrayCGH and WES of CTCs and will be expanded upon further in year 5 of the grant application.

Task 6: To develop skills necessary to succeed as a leader in clinical and translational research and obtain independent peer-reviewed research funding to support a long term career in research (Months 1-60)

Dr. Armstrong has formed a mentoring and collaboration team comprised of Mariano A. GarciaBlanco MD PhD, Daniel J George MD, Simon Gregory PhD, and James Abbruzzesse MD for the purposes of facilitating career development on a clinical research tenure track at Duke and 
within the Duke Cancer Institute. In May 2014, he was promoted to Associate Professor of Medicine and Surgery with tenure. He has joined a peer-reviewed mentoring group for clinical translational researchers led by Christopher Kontos PhD, an established cardiology translational researcher, which holds monthly meetings on topics such as mentoring, conflict resolution, team building, grant writing, and collaboration. He attended a GOPEN session on biomedical writing (grant writing). Finally Dr Armstrong established a Molecular Medicine Scholar Program in Oncology that he co-leads, which facilitates mentoring and training of graduate laboratory and post-doctoral students (PhD) in clinical and translational oncology. This training program brings together 5-6 trainees from Duke basic science departments who are focused on oncology research, and provides networking, clinical rotations (inpatient and outpatient), mentorship, and didactic seminars with experienced cancer researchers. Through these experiences, Duke has provided an excellent opportunity to be both mentored and to mentor in a translational setting.

Dr Armstrong is a full Duke IRB board member and a member of the Duke Cancer Institute's Cancer Protocol Committee, which provides scientific review pre-IRB review for cancer protocols. This committee meets monthly to review all protocols in cancer prior to IRB review for scientific merit. This practice-based training in clinical research is ongoing and provides much needed experiential education in the conduct of clinical trials and research design and safety oversight.

Dr. Armstrong was appointed as the co-director for clinical and translational research in GU oncology within the newly formed Duke Cancer Institute, overseeing the clinical trial and translational aspects of research in prostate cancer and other GU malignancies at Duke.

Dr. Armstrong participates regularly in a number of educational and research based meetings at Duke, including the prostate cancer journal club (monthly), clinical research staff meetings (oversight of trial conduct, data and safety monitoring, weekly), the Garcia-Blanco laboratory meetings (weekly to monthly), the prostate cancer research (monthly), new protocol meetings (to review internal and external proposals, monthly), Translational Science meetings through the Department of Pharmacology and Cell Biology (monthly), Oncology Grand Rounds (weekly), urology tumor board (monthly). He runs a monthly journal club on cancer metastasis biology. Dr. Armstrong participated in 2010 in a Duke Clinical Research Leadership Retreat (3 days), sponsored by the Dean's Office, which provided valuable feedback and education in a range of leadership topics from conflict resolution to giving feedback to leading a diverse team of staff and peers. Dr. Armstrong serves on the oncology fellowship committee, and is now a mentor to a medical oncology fellow and emerging faculty member (Rhonda Bitting), who is also working on these CTC biology projects. He is an ongoing mentor to two post-doctoral medical oncology fellows starting July 1, 2013, Drs. Tian Zhang and Jing Li, who will be working with him on a variety of CTC and plasticity projects in prostate cancer.

He also served as a mentor to Duke undergraduates in the Garcia-Blanco lab who shadow him in the clinic as they develop methods to study epithelial plasticity and metastasis in the laboratory. He serves as a clinical mentor to two post-doctoral fellows in the Garcia-Blanco laboratory, Drs. Jason Somarelli and Daneen Schaeffer, each of whom works on cancer metastasis and plasticity biology as it relates to FGFR2 signaling and alternative splicing. He mentors Kathryn Ware, $\mathrm{PhD}$ in her post-doctoral fellowship work investigating the relationship of epithelial plasticity to androgen receptor variant levels and enzalutamide resistance.

In addition, Dr Armstrong participates in the following national-level programs that provide training in leadership and clinical practice: ASCO (Chair of a Clinical Science Symposium in 2012), NCCN (Prostate Cancer guidelines member), GU Symposium (ASCO-ASTRO-SUO), and the Prostate Cancer Foundation annual scientific retreat. He was co-chair of the 2012 PCF Retreat Session on EMT in Prostate Cancer. He attended the DoD IMPACT meeting in 2011 as well and presented an educational talk on "Prostate Cancer 101" in addition to presenting several posters and leading a walking tour of the posters. He was selected as a grant reviewer 
for the Prostate Cancer Challenge Awards and Mazzone PCF grant mechanism (High Impact Award). He is a reviewer for numerous oncology and medical journals, which provides training and experience in scientific review. He intends to apply for several major grants in 2014, including DOD awards (Synergy and the PCCTC consortium), as well as a multi-PI R01 award with Dr. Garcia-Blanco. In addition, he received in July 2104 a large multiyear multiinvestigator PCF Global Treatment Sciences Challenge award based on the CTC biomarker preliminary data described above. In year 5, Dr. Armstrong will continue with these educational and scientific endeavors as part of his clinical research training and academic mission. 
KEY RESEARCH ACCOMPLISHMENTS-YEAR 4 (See Years 1-2 for additional accomplishments)

- Discovery of the lack of prognostic importance of mesenchymal biomarker expression in men with localized prostate cancer treated with prostatectomy.

- Validation of proliferation (Ki-67) as an independent prognostic biomarker in localized prostate cancer for PSA recurrence.

- Identification of a novel circulating tumor cell capture method to enable capture and characterization of a more mesenchymal CTC population defined by N-cadherin, OBcadherin, or c-met expression. This method has led to the identification of rare cells clonally derived from epithelial prostate cancer cells that lack cytokeratin and express OB-cadherin and beta-catenin. Testing of the N-cadherin and c-met CTC capture method is ongoing.

- Development of methods to permit the whole exome sequence and copy number profiles (amplifications/deletions) to be identified reliably from CTCs in men with mCRPC.

- Identification of loss of AR and gain of neuroendocrine biomarkers such as MYCN during enzalutamide resistance, as determined by the molecular profiling of CTCs using arraybased comparative genomic hybridization (arrayCGH)

- Identification of multiple single nucleotide polymorphisms and small insertions/deletions present in CTCs from a patient with $\mathrm{mCRPC}$ who was resistant to enzalutamide, including a number of gene mutations implicated in cancer biology and AR biology.

- Receipt of a PCF Global Treatment Sciences Challenge Award (PI Armstrong) 20142016 based on preliminary data achieved with this DOD PRTA 
1. Bitting RL, Boominathan R, Rao C, Kemeny G, Foulk B, Garcia-Blanco MA, Connelly M, Armstrong AJ. Development of a method to isolate circulating tumor cells using mesenchymal-based capture. Methods. 2014 (Dec), 64: 129-136.

2. Armstrong AJ, Healy P, Halabi S, Vollmer R, Kemeny G, Ware K, Freedland SJ. Evaluation of an Epithelial Plasticity (EP) Biomarker Panel with Prognosis in Men with Localized Prostate Cancer. Clin Cancer Res 2014 (submitted). See Appendix 1.

3. Clinical СTC paper submitted to Prostate Cancer Prostatic Diseases: Bitting RL, Healy, Halabi S, George DJ, Armstrong AJ. Clinical Phenotypes Associated with Circulating Tumor Cell Enumeration in Metastatic Castration Resistant Prostate Cancer. Prostate Cancer and Prostatic Dis (submitted). See Appendix 2.

4. Bitting RL, Schaeffer D, Somarelli JA, Garcia-Blanco MA, Armstrong AJ. The role of epithelial plasticity in prostate cancer dissemination and treatment resistance. Cancer Metastasis Rev 2014; Jan 11. See Appendix 3.

5. Ware KE, Garcia-Blanco MA, Armstrong AJ, Dehm S. Significance of androgen receptor variants in castration resistant prostate cancer. Endocr Relat Cancer 2014 (May 23 epub ahead of press). See Appendix 4.

6. Awarded funding by the Prostate Cancer Foundation Global Treatment Sciences Challenge Award, July 2014 (\$1 million).

7. Ongoing work as co-chair of the Prostate Cancer Working Group 3, with Howard Scher MD of Memorial Sloan Kettering Cancer Center, anticipated publication in 2015.

8. Ongoing member of the NCCN National Prostate Cancer Guidelines panel

9. US Patent: revised $6 / 2013$ for the detection of circulating tumor cells based on EMT antigen-based capture

10. Research Collaboration Agreement: Ongoing with Veridex/Janssen to develop a next-generation CTC detection assay based on our preliminary data and published data from this PRTA. This has included the provision and development of a research Cellsearch $®$ machine and Cell tracks $®$ Analyzer and two novel ferrofluids for clinical testing. This collaboration was renewed through 2016.I

11. Additional Grant proposals that arose from this award: DOD New Idea Award (funded 2012-2014), R01 (co-PI Garcia-Blanco and Armstrong), not funded: Alternative splicing and epithelial-mesenchymal plasticity in prostate tumors, submitted March 2013. R21 (PI Pei Zhong) for Tandem bubble-SAW technology for viable isolation and characterization of CTCs, start date 9/2014 (pending). 


\section{CONCLUSIONS}

In this fourth annual report for the DOD PRTA, we have 1) provided strong evidence for the existence of epithelial-mesenchymal transition in human cancer, particularly metastatic castration resistant prostate cancer; 2) provided preliminary data supporting the ability to molecularly profile CTCs with both array-based CGH and whole exome sequencing; and 3) developed methods to permit high-throughput DNA, RNA, and protein-based biomarker assessments of CTCs from men with MCRPC. These findings are based on the discovery of EMT and stemness biomarker expression in CTCs from men with CRPC and for the existence of a novel CTC phenotype based on EMT-biomarker (OB-cadherin) capture. The findings of EMT and stemness markers in the majority of circulating tumor cells from men with metastatic prostate cancer and women with metastatic breast cancer has several important implications: 1) EMT and stemness pathways likely play an important role in cancer progression, metastasis, and lethality and suggests routes for new therapeutic approaches; and 2) current methods to detect CTCs that rely solely on epithelial characteristics (i.e. EpCAM) may miss a large number of non-epithelial tumor cells that have undergone EMT during circulatory spread and transit. The detection of OB-cadherin positive cells in men with $\mathrm{MCRPC}$ is novel and intriguing and suggests that these bone-homing cells may be undergoing osteomimicry during EMT and that this plasticity may be contributing to bone metastasis and the lethal clinical phenotype. In some of these cells, we have clearly identified by FISH the presence of PC-specific genetic alterations. In year 5, we will further refine this mesenchymal marker capture using novel OBcadherin kits and c-met based capture methods. We have also developed methods using whole genome amplification, and whole exome and RNA Sequencing to investigate molecular aberrations in CTCs from men with CRPC, and will be applying these methods in years 4-5 to the prospective identification of druggable (actionable) targets in real time in men with mCRPC. We will further the application of our findings directly toward therapeutic target identification and technology development through the newly funded PCF Global Challenge Award which will take place across multiple DOD PCCTC institutions, and which leveraged the preliminary data from this PRTA into a successful long term proposal. By identifying novel cell phenotypes and pathways that contribute to lethal CRPC progression, our hope is to use these CTC biomarkers early to predict therapeutic success with novel hormonal therapies and taxane chemotherapies, and to develop and annotate novel targets that evolve or emerge during clonal selection or tumor adaptaption for future therapy in men with CRPC. 
1. Siegel R, Naishadham D, Jemal A. Cancer statistics, 2013. CA Cancer J Clin 2013;63(1):11-30.

2. de Bono JS, Scher HI, Montgomery RB et al. Circulating tumor cells predict survival benefit from treatment in metastatic castration-resistant prostate cancer. Clin Cancer Res 2008;14(19):6302-6309.

3. Oltean S, Sorg BS, Albrecht $\mathbf{T}$ et al. Alternative inclusion of fibroblast growth factor receptor 2 exon IIIc in Dunning prostate tumors reveals unexpected epithelial mesenchymal plasticity. Proc Natl Acad Sci U S A 2006;103(38):14116-14121.

4. Armstrong AJ, Marengo MS, Oltean S et al. Circulating Tumor Cells from Patients with Advanced Prostate and Breast Cancer Display Both Epithelial and Mesenchymal Markers. Mol Cancer Res 2011.

5. Beltran H, Rickman DS, Park K et al. Molecular characterization of neuroendocrine prostate cancer and identification of new drug targets. Cancer Discov 2011;1(6):487495.

6. Greuber EK, Smith-Pearson P, Wang J, Pendergast AM. Role of ABL family kinases in cancer: from leukaemia to solid tumours. Nat Rev Cancer 2013;13(8):559-571. 
A. Manuscript: tissue microarray EMT biomarker paper (submitted to Clinical Cancer Research)

B. Manuscript: Clinical CTC paper (submitted to Prostate Cancer and Prostatic Diseases)

C. Manuscript: Epithelial Plasticity in Prostate Cancer (Cancer Metastasis Reviews 2014).

D. Manuscript: Biologic and Clinical Significance of Androgen Receptor Variants in Castration Resistant Prostate Cancer (Endocrine Rel Cancer 2014).

E. Abstract: GU ASCO 2014

F. Current CV 2014 


\title{
Evaluation of an Epithelial Plasticity (EP) Biomarker Panel with Prognosis in Men with Localized Prostate Cancer
}

\author{
Andrew J Armstrong ${ }^{1,6^{*}}$, Patrick Healy ${ }^{2,6}$, Susan Halabi ${ }^{2,6}$, Robin Vollmer ${ }^{4}$, Gabor \\ Kemeny ${ }^{5-6}$, Kathryn Ware ${ }^{5}$, and Stephen J. Freedland ${ }^{3}$ \\ ${ }^{1}$ Department of Medicine/Division of Medical Oncology, \\ ${ }^{2}$ Department of Biostatistics and Bioinformatics, \\ ${ }^{3}$ Department of Surgery/Division of Urology, Duke University and the Durham VA \\ Medical Center \\ ${ }^{4}$ Department of Pathology, Duke University and the Durham VA Medical Center \\ ${ }^{5}$ Department of Molecular Genetics and Microbiology \\ ${ }^{6}$ Duke Cancer Institute, Duke University Medical Center, Durham, NC 27710 USA
}

Running title: Epithelial Plasticity Biomarkers in Localized Prostate Cancer

Keywords: epithelial to mesenchymal transition, prostate cancer, tumor biomarkers, prognosis, PSA recurrence

Financial support: Prostate Cancer Foundation Young Investigator Award (AJA) and Department of Defense grant W81XWH-10-1-0483 (AJA)

*Correspondence:

Andrew Armstrong, DUMC Box 102002, Durham NC 27710

andrew.armstrong@duke.edu

Conflict of interest: none

Word count:

Number of figures: 4

Number of tables: 3 


\section{Translational relevance:}

Epithelial plasticity (EP), referring to the loss of an epithelial phenotype, is a biologic process that is thought to occur during solid tumor invasion and promote metastatic dissemination and therapeutic resistance in many tumor types including prostate cancer. This reduction of epithelial phenotypic biomarkers may be accompanied by an increase in mesenchymal biomarkers through an epithelial to mesenchymal transition in the primary tumor, and the reverse process of mesenchymal to epithelial transition in metastatic colonies. Here we have investigated a panel of EP biomarkers in over 200 men with localized prostate cancer treated with radical prostatectomy and who have long-term follow up. We did not identify any EP biomarkers associated with aggressive or recurrent disease, and found that the predominance of aggressive prostate cancers were epithelial in nature when evaluated in the primary tumor. While this does not rule out a rare or geographically isolated cellular plasticity event contributing to metastasis, it illustrates that the majority of prostate cancer cells in the prostate are epithelial in nature, and that a macroscopic mesenchymal biomarker assessment does not add independent clinical value to standard clinical/pathologic risk assessment. 


\begin{abstract}
Purpose: Given the potential importance of epithelial plasticity (EP) to cancer metastasis, we sought to investigate biomarkers related to EP in men with localized prostate cancer (PC) for the association with clinical outcomes after surgery.

Experimental Design: Men with localized PC treated with radical prostatectomy at the Durham VA medical center and whose prostatectomy tissues were included in a tissue microarray (TMA) linked to long-term outcomes. We performed immunohistochemical studies using validated antibodies against E-cadherin and Ki-67 and mesenchymal biomarkers including N-cadherin, vimentin, SNAIL, ZEB1, and TWIST. Association studies were conducted for each biomarker with baseline clinical/pathologic characteristics and risk of recurrence over time.

Results: Two hundred and six men contributed TMA tissue and had long-term followup (median 11 years). Forty-three percent had PSA recurrence; 3 men died of PC. The majority of PC had high E-cadherin expression (86\%); 14\% had low/absent E-cadherin expression. $\mathrm{N}$-cadherin was rarely expressed $(<4 \%)$ and we were unable to identify an $\mathrm{E}$ to $\mathrm{N}$ cadherin switch as prognostically important. No associations with risk, outcome, or adverse pathologic characteristics were noted for SNAIL, ZEB1, vimentin, or TWIST, despite heterogeneous expression. We observed an association of higher Ki-67 expression with Gleason sum, NCCN risk, and PSA recurrence (HR 1.08, $p=0.0095)$.
\end{abstract}

Conclusion: Localized PC, including high risk disease, has a predominantly epithelial, not mesenchymal phenotype. The expression of EP biomarkers in localized disease in this cohort of men with a low risk of PC-specific mortality was not associated with aggressive features or PSA relapse after surgery. 


\section{Background}

Localized prostate cancer $(P C)$ is a heterogeneous disease, in which men have widely disparate outcomes based on a number of clinical and pathologic factors including Gleason sum, PSA levels, tumor stage, and the extent of invasion(1, 2). Current models of risk of recurrence or PC mortality after surgery are reasonably accurate at assessing long term outcomes(1). However, some low and intermediate risk tumors still relapse while some high risk tumors may be cured with surgery alone and our ability to predict these discordant results is imperfect and illustrating the biologic heterogeneity even within well-defined risk categories(3). Thus, additional biomarkers of biologic aggressiveness in localized PC are needed.

Epithelial plasticity (EP), defined as the ability of cells to reversibly undergo phenotypic changes, may underlie the ability of many solid tumors, including PC, to disseminate and resist commonly used therapies, including surgery, radiation, hormonal therapies, and chemotherapy $(4,5)$. During the loss of the more differentiated epithelial phenotype, cancer cells may up-regulate stemness biomarkers(6) or biomarkers of a mesenchymal or invasive phenotype(7), associated with an epithelial-to-mesenchymal transition (EMT). An EMT has been associated with metastatic risk in multiple tumor types, and prostate cancer cell lines and human metastases expressing EMT biomarkers appear to be more androgen receptor independent and aggressive(8). We have shown that circulating tumor cells (CTCs) from men with metastatic castration resistant prostate cancer commonly express these plasticity biomarkers, indicating their potential importance in lethal disease(9), and others have shown that loss of epithelial 
biomarkers and/or an increase in mesenchymal or stemness biomarkers in localized PC may be associated with recurrent disease and $\mathrm{PC}$ mortality $(6,7,10)$.

Several studies have specifically analyzed mesenchymal biomarker expression in radical prostatectomy specimens, identifying an E- to N-cadherin switch(7), loss of cytokeratin or PSA expression(6, 11), gain of hedgehog or NOTCH signaling(6), or gain of expression of the EMT transcriptional regulators TWIST and SNAIL(10), as adversely prognostic and independently associated with recurrent disease. However, others have not found associations between SNAIL or vimentin expression $(12,13)$, and currently EP biomarkers are not routinely assessed during the pathologic examination of the prostate. We thus sought to evaluate the association of EP biomarker expression in a large series of men with localized PC with long-term outcomes.

\section{Materials and Methods}

Patient population

The current cohort includes men with localized prostate cancer treated with radical prostatectomy between 1993-2004 at the Durham Veteran's Affairs Medical Center (VAMC) in Durham NC. Clinical data was extracted and included in the Shared Equal Access Research Center Hospital (SEARCH) database. Data recorded included age, demographics, PSA levels at diagnosis and recurrence, prostatectomy pathologic characteristics, stage and NCCN risk score, prior and subsequent therapies, biopsy information, and long term recurrence, metastasis, and survival outcomes. A tissue microarray on a subset of patients in the SEARCH database treated at the Durham VA 
was developed after institutional review board approval in which prostatectomy histologic sections were arrayed on slides for biomarker evaluation, along with benign negative control tissues.

\section{Antibodies and validation}

We performed antibody optimization and validation for all antibodies tested, determining the optimal concentration using both negative and positive control tissues prior to application to the TMA. Antibodies against E-cadherin, Ki-67, N-cadherin, vimentin, SNAIL1/2, TWIST, and ZEB1 were evaluated in parallel with hematoxylin and eosin $(\mathrm{H} \& \mathrm{E})$ by an expert prostate cancer pathologist blinded to outcomes and other biomarker results. Scoring of each biomarker followed an ordinal scale ranging from 02 or $0-3$ based on intensity and frequency of expression in each TMA section. Ki-67 was scored on a $0-100 \%$ scale based on frequency of expression in tumor cells. Each patient contributed multiple TMA sections from radical prostatectomy in a random TMA which was then linked back to the subject ID by a master code for clinical database association studies. For each biomarker, minimum and maximum expression per subject as well as average expression was associated with outcomes and pathologic/clinical features. Scoring of epithelial tumor cells rather than benign stroma was performed for all EP biomarkers. Table 1 provides a listing of each antibody used, the source and clone, isotype, positive and negative controls, and concentrations used. 
Statistical methods and analysis plan

The primary endpoint was to determine the association of each EP biomarker with PSA recurrence over time. Secondary objectives included the association of each EP biomarker with adverse clinical/pathologic characteristics (PSA, Gleason sum, NCCN risk, stage, survival and risk of metastasis), and each biomarker with each other. Frequency tables were generated for each marker with at least marginal variation across both demographic factors of interest and the other TMA markers. Pearson chisquare or Fisher's Exact Test were used as appropriate. P-values are preliminary as no adjustment was made for multiple comparisons. Overall survival (OS) was computed using the variables 'limbo' and 'dead.' Patients who had not failed as of last follow up were censored at time of last follow up. Median survival, Kaplan Meier plots, and univariate hazard ratios are reported. Patients who had not recurred as of last follow up, including those who expired prior to recurring, were censored at time of last follow up or death. Median time to recurrence based on PSA, Kaplan Meier plots, and univariate hazard ratios are reported.

\section{Results}

All 205 men with localized PC were included. The median age was 63 years (range $47-73$ ). Fifty percent of men were White and $48 \%$ of men were Black. By D'Amico/NCCN risk classification, $29 \%$ were low risk, $38 \%$ intermediate risk, and $21 \%$ high risk. At surgery, 12.2\% had Gleason 8-10 tumors, 67.3\% were Gleason 7, and $20.5 \%$ were Gleason 6 or under. Fifteen percent had seminal vesicle invasion, $27 \%$ had extracapsular extension, and PSA at the time of surgery was a median of 7.4 (range 0.6-75.4). Over a follow-up period of 11.29 years, 71 (34.6\%) men died, with 3 
(1.4\%) dying of prostate cancer and 4 men (2\%) developing metastatic prostate cancer. Eighty-nine (43.4\%) had biochemical (PSA) recurrence and $37.6 \%$ of men were treated with adjuvant or salvage radiation to the prostate bed. Fifteen percent of men required androgen deprivation therapy (ADT) at any time; however, no patients received ADT prior to surgery. Table 2 provides these demographic and clinical characteristics.

We initially examined Ki-67 as validated biomarker of prognosis and tumor proliferation rate and as a positive control biomarker for our TMA studies, given the association of higher Ki-67 expression with recurrence and adverse pathology in multiple prior studies (14-18). In our TMA study, the average Ki-67 expression per patient was 3.1\% (range $0-18.7 \%$ ), while the maximum Ki-67 expression per patient was $4.7 \%$ (range $0-21.7 \%$ ), and was evaluable in 178 of the 205 men. Of these 178 , we identified 14 men who had Ki-67 scores of 0 , in which benign prostate tissue was likely scored in the present study, leaving 164 men evaluable for Ki-67 analysis. Using the average $\mathrm{Ki}-67$ score $(0-100 \%$ range $)$ as a continuous variable, $\mathrm{Ki}-67$ percentage was associated with PSA recurrence (HR 1.09 for each unit increase in Ki-67, $p=0.0095, H R$ 1.02-1.16). This remained significant if the men with 0 scores for Ki-67 were included (HR $1.06 \mathrm{p}=0.0495)$. In multivariate analysis adjusting for NCCN risk, Ki67 remained associated with PSA recurrence (HR 1.09, 95\% Cl 1.02-1.16, $p=0.0118)$, and NCCN risk was also associated with PSA recurrence (HR 1.92 and 2.24 for high and intermediate vs. low risk, respectively, $p=0.047$ and 0.0025$)$. However, this risk was not uniform across all Ki-67 quartiles, as shown in Figure 1a. The time to PSA recurrence was shortest in the two highest quartiles (median 8.1 and 3.4 years for 
quartiles 3 and 4) as compared to the lowest two Ki-67 quartiles (median 9.9 and not reached for quartiles 1 and 2). The probability of being free of PSA recurrence at 5 years was $67.4 \%, 69.8 \%, 57.4 \%$, and $43.6 \%$ in the first, second, third, and fourth quartiles of average Ki-67 expression. Figure 1b demonstrates the outcomes of our patients by NCCN risk score (low, intermediate, high). While patients with low risk prostate cancer had favorable outcomes $(73 \%$ of men were free of PSA progression at 5 years, median time to recurrence not yet reached), men with intermediate or high prostate cancer had a higher risk of recurrence ( 49 and $45 \%$ of men free of PSA progression at 5 years, respectively, and median time to recurrence of 4.9 and 4.6 years). The differences in PSA recurrence over time across NCCN risk groups was statistically significant $(\log$ rank $p=0.0057$ and 0.0005 for low vs. high and low vs. intermediate risk, respectively).

Having validated Ki-67 and NCCN risk in our TMA-SEARCH clinical dataset, we next evaluated a series of EP biomarkers in primary prostate cancer tissues. Examples of representative $\mathrm{IHC}$ images of each biomarker are shown in figure $\mathbf{2}$ and supplementary figure 1 . We found that the majority of prostate cancers had an epithelial phenotype. For example, only 1.5 and $12.7 \%$ of tumors had absent or low Ecadherin expression; the majority (85.6\%) had high E-cadherin expression. Of evaluable tissue, $\mathrm{N}$-cadherin was rarely expressed, and was present in only $3.9 \%$ of prostate cancers, while $96.0 \%$ had absent $\mathrm{N}$-cadherin expression. We found vimentin expression to be largely stromal in distribution and only tumor cell vimentin was scored. We found that $22.4 \%$ and $5.9 \%$ of tumors expressed intermediate and high vimentin, respectively, and most cancers had absent vimentin expression (70.7\%). Zeb1 was 
expressed in $15.3 \%$ of cases, with only 3 cases demonstrating intense staining. SNAIL was expressed more heterogeneously, with $31 \%, 39 \%, 24 \%$, and $5 \%$ of prostate cancers having $0,1,2$, and 3+ SNAIL expression, respectively. Finally, TWIST expression was also heterogeneously expressed, with $1 \%, 6 \%, 31 \%$, and $69 \%$ of prostate cancers having $0,1,2$, and $3+$ TWIST expression.

In univariate analysis, we found no association with any EP biomarker and PSA recurrence (table 3). When examined using the average expression, minimum expression, or maximum expression, we found no associations with outcome for low Ecadherin, high N-cadherin, high SNAIL, high vimentin, high ZEB1, or high TWIST expression. In several cases, lower mesenchymal protein expression was numerically associated with improved outcome, although this was not statistically significant. For example, men with high levels of SNAIL expression had a 5 year probability of being free of PSA progression of $69 \%$ as compared to $53 \%$ for men with low SNAIL expression in their prostate cancer. Similar trends were seen for vimentin (5 year PSA recurrence-free probability of $68 \%$ for high vimentin, vs $57 \%$ for absent vimentin). High E-cadherin was associated with a greater probability of PSA relapse at 5 years (43\%) as compared to men with low to absent E-cadherin in their prostate cancer ( $24 \%$ risk of PSA relapse). N-cadherin was not evaluated for associations with PSA recurrence given the low number of men who had high $\mathrm{N}$-cadherin expression $(\mathrm{n}=8)$. TWIST expression was also not associated with PSA relapse (table 3). Given the low number of PC-specific deaths or metastases, we were unable to evaluate the associations of EP biomarkers with these endpoints despite the relatively long follow-up period. 
We next examined whether EP biomarkers were associated with clinical/pathologic risk factors related to prostate cancer prognosis. The expression of Ki-67 was directly linked to NCCN risk groups, with $40 \%, 53 \%$, and $69 \%$ of low, intermediate, and high risk cases having Ki-67 expression levels over the median value ( $p=0058$ by Fisher exact test). The median Ki-67 expression (using average score across the TMA for each subject) was 2.4, 3.1, and 5.2 percent for low, intermediate, and high NCCN risk ( $p=0.0423$ by Kruskal-Wallis test, Figure 3 ). Plots of the proportion of men with high Ki-67 (>median), TWIST (2-3+), SNAIL (2-3+), vimentin (1-2+), and ZEB1 (1-2+) and low E-cadherin (0-1+) expression according to NCCN/classic D'Amico risk groups are shown in figure 3. While Ki67 significantly increased with NCCN risk, we did not observe any statistically significant associations of EP biomarkers with increasing NCCN risk. We did observe an increase in ZEB1 expression with increasing NCCN risk group which was not statistically significant. However, ZEB1 was not associated with Gleason sum at surgery, risk of PC recurrence, or death (HR 0.80, $p=0.52$ ). Other mesenchymal biomarkers decreased with NCCN risk (vimentin) or had no association with risk group (SNAIL, loss of E-cadherin, TWIST, N-cadherin).

\section{Discussion}

In this study, we examined the association of a range of epithelial plasticity biomarkers for their prognostic association with PSA recurrence in a contemporary series of men with localized prostate cancer treated with curative intent radical prostatectomy. The outcomes in this cohort of VA men were excellent, with only 3 deaths from prostate cancer and 4 patients developing metastatic disease despite a 
$43 \%$ PSA recurrence rate. While a biomarker of proliferation, Ki-67, was validated as being associated with $\mathrm{NCCN}$ risk and risk of PSA relapse after surgery, we found no associations of EP biomarkers with clinical risk groups or PSA recurrence in our study.

There are several possible explanations for why our results do not confirm previous work suggesting a link between mesenchymal biomarker expression in localized prostate cancer and outcomes after surgery $(7,10,19)$. The first is the overall excellent long term outcomes in our patient population, limiting our ability to demonstrate an association of any biomarker with prostate cancer metastasis or death due to low event rates and power. This reflects the improving prognosis of men treated for localized prostate cancer over time, and the limitations of any biomarker for improving upon standard clinical risk stratification in a contemporary series above and beyond stage, grade, and baseline PSA. In addition, we could not validate the E- to Ncadherin switch as prognostic given the low level $\mathrm{N}$-cadherin expression observed in our relatively low risk cohort of men. While the E- to $\mathrm{N}$-cadherin switch data(7) has not yet been validated externally, it may still be possible to validate this poor prognostic finding in a larger cohort of higher risk men followed long term through metastatic relapse and death.

Second, we found several mesenchymal biomarkers to be quite commonly expressed in low grade prostate cancers. For example, loss of E-cadherin or low Ecadherin expression was more commonly seen in low grade Gleason 6 disease, while most high grade tumors had abundant and intense E-cadherin staining. Similarly, TWIST expression was ubiquitous across NCCN risk groups, and vimentin expression in prostate tumor cells actually decreased with increasing grade. SNAIL and ZEB1 
expression was not associated with grade or clinical risk in our series. These data suggest that localized prostate cancer, especially high grade/high risk tumors, have a more epithelial phenotype in the tumor bulk, and that broad mesenchymal biomarker expression in high grade disease is not common or associated with outcomes.

There are several limitations present in our study. The first includes the lack of rare cell isolation within tumors that lack epithelial biomarker expression or have mesenchymal biomarker over-expression. These alternative and more complex histologic methods, using quantitative imaging and dual-color immunofluorescence, have associated a loss of PSA expression or cytokeratin staining with high grade, poor risk disease and adverse outcomes after $\operatorname{surgery}(6,11)$. Second, a TMA is unable to assess regional or geographic variability of biomarkers, for example mesenchymal biomarker expression only at the invasive tumor front. Our TMA did not include this geographic distribution information, and future studies should consider annotation of biomarker expression according to geographic distribution (central, peripheral, invasive strands, capsular invasion regions) in order to better ascertain the relationship of a given biomarker distribution with outcome, similar to that described in other solid tumors such as colorectal cancer(20). Given the multifocal and heterogeneous nature of prostate cancer, care will need to be taken to describing this distribution according to tumor site and grade $(21,22)$.

Finally, our cohort of men was relatively small and despite the long term followup, the number of metastatic and prostate cancer specific mortality events was low, limiting our power to observe the associations of EP biomarkers with these clinically relevant outcomes. Our prior work suggested the common presence of these EP 
biomarkers in circulating tumor cells (CTCs) from men with castration-resistant prostate cancer (CRPC), suggesting the importance of EP with lethal forms of the disease $(4,9$, 23). Others have confirmed these findings, but suggested that these EP biomarkers may be less prevalent in hormone-sensitive disease(24). There are data suggesting that castration itself may promote an epithelial to mesenchymal transition, possibly linked to the development of androgen receptor alterations such as splice variants(25-27). Our cohort of men were all unexposed to androgen deprivation therapy (ADT) and had a low metastatic rate, which may limit our ability to detect an EMT if epithelial plasticity is more relevant to castration-resistant progression and/or metastasis. Only $12 \%$ of our patients had high grade Gleason sum 8-10 disease, which limits our ability to determine associations within this high risk subpopulation. Nevertheless, we observed that the majority of high grade tumors had an epithelial phenotype, and that mesenchymal biomarker expression was often more commonly expressed in lower risk cancers. These data suggest the importance of a mesenchymal to epithelial transition (MET) in prostate cancer, or that the majority of primary prostate cancers have largely not undergone an EMT, at least in the majority of their cells. We cannot rule out rare cellular events relevant to EMT and cancer dissemination in this study, however.

In conclusion, while we validated $\mathrm{Ki}-67$ as an independent biomarker of prostate cancer aggression and recurrence after surgery, we found that the majority of localized prostate cancers were epithelial in phenotype, and that mesenchymal biomarker expression was not associated with relapse. These data suggest that knowledge of epithelial plasticity biomarker expression in the tumor bulk in men with localized prostate cancer may not add clinical utility to our current clinical measures of prognosis. Further 
study of rare de-differentiated cellular populations and geographic distribution of EP biomarkers in prostate cancer is warranted. 
Table 1. Antibodies used for immunohistochemical studies performed in the present study. 


\begin{tabular}{|c|c|c|c|c|c|c|c|c|}
\hline Antigen & $\begin{array}{l}\text { Antibody } \\
\text { Source }\end{array}$ & $\begin{array}{l}\text { Catalog/ } \\
\text { clone ID }\end{array}$ & Host & Isotype & Dilution & Retrieval & $\begin{array}{c}\text { Positive } \\
\text { Control } \\
\text { Tissue }\end{array}$ & $\begin{array}{c}\text { Negative } \\
\text { Control } \\
\text { Tissue }\end{array}$ \\
\hline Vimentin & Dako & M7020 & $\mathrm{M} / \mathrm{m} / \mathrm{aH}$ & $\operatorname{lgG} 2 a$ & $1: 150$ & $\begin{array}{c}\text { (0.5\% in } 5 \mathrm{mM} \mathrm{HCl}) \text { Pepsin, } \\
40 \mathrm{C}^{\circ} / 15 \text { mints }\end{array}$ & $\begin{array}{l}\text { Tonsil, } \\
\text { Kidney }\end{array}$ & $\begin{array}{c}\text { Internal } \\
\text { stroma } \\
\text { control }\end{array}$ \\
\hline E Cadherin & Dako & M3612 & $\mathrm{M} / \mathrm{m} / \mathrm{aH}$ & $\operatorname{lgG1(k)}$ & $1: 100$ & $\begin{array}{c}\text { 10mMTris Base, 1mM EDTA, 0.05\% } \\
\text { Tween20, pH } 9.0\end{array}$ & $\begin{array}{l}\text { Breast } \\
\text { Cancer }\end{array}$ & $\begin{array}{c}\text { Internal } \\
\text { epithelial } \\
\text { control }\end{array}$ \\
\hline N Cadherin & Dako & M3613 & $\mathrm{M} / \mathrm{m} / \mathrm{aH}$ & $\operatorname{lgG1}(k)$ & $1: 50$ & $\begin{array}{c}10 \mathrm{mM} \mathrm{Na} \text { Citrate / pH6.1 } \\
95 \mathrm{C}^{\circ} / 20 \mathrm{mints}\end{array}$ & Heart & $\begin{array}{c}\text { Internal } \\
\text { stroma } \\
\text { control }\end{array}$ \\
\hline Ki67 & Dako & M7240 & $\mathrm{M} / \mathrm{m} / \mathrm{aH}$ & $\operatorname{lgG1}(\mathrm{k})$ & $1-50$ & $\begin{array}{c}\text { 10mM NaCitrate/0.05\%Tween } 20 \\
\text { pH6.1, 95 } \mathrm{C}^{\circ} / 20 \text { mints }\end{array}$ & Tonsil & $\begin{array}{l}\text { Benign } \\
\text { tissue }\end{array}$ \\
\hline Zeb1 & NovusBio & NBP1-88854 & $\mathrm{R} / \mathrm{p} / \mathrm{aH}$ & $\lg G$ & $1: 250$ & $1 \mathrm{mM}$ EDTA pH 8.0, 95 $\mathrm{C}^{\circ} / 20 \mathrm{mints}$ & $\begin{array}{l}\text { Breast } \\
\text { Cancer }\end{array}$ & $\begin{array}{c}\text { Normal } \\
\text { breast } \\
\text { Tissue }\end{array}$ \\
\hline Snail/Slug & Abcam & Ab85936 & $\mathrm{R} / \mathrm{p} / \mathrm{aH}$ & $\lg G$ & $1: 500$ & $1 \mathrm{mM}$ EDTA pH 8.0, 95 $\mathrm{C}^{\circ} / 20 \mathrm{mints}$ & $\begin{array}{l}\text { Breast } \\
\text { Cancer }\end{array}$ & $\begin{array}{c}\text { Normal } \\
\text { breast } \\
\text { tissue }\end{array}$ \\
\hline Twist & Abcam & Ab49254 & $\mathrm{R} / \mathrm{p} / \mathrm{aH}$ & $\lg G$ & $1: 100$ & $\begin{array}{c}\text { 10mM NaCitrate / pH6.1 } \\
95 \mathrm{C}^{\circ} / 20 \text { mints }\end{array}$ & Testis & $\begin{array}{l}\text { Normal } \\
\text { Prostate }\end{array}$ \\
\hline
\end{tabular}


Table 2. Baseline characteristics of the patients included in the present tissue microarray study. ADT=androgen deprivation therapy. PSA=prostate specific antigen. $\mathrm{NCCN}=$ National Comprehensive Cancer Network.

\begin{tabular}{|r|c|}
\hline $\begin{array}{c}\text { Clinical and Pathologic } \\
\text { Characteristics of the Patients }\end{array}$ & N (\%), range \\
Age, years & $63(47-73)$ \\
Race/Ethnicity & \\
White (\%) & $104(50.7 \%)$ \\
Black (\%) & $99(48.3 \%)$ \\
Hispanic (\%) & $0(0 \%)$ \\
PSA at the time of surgery, ng/dl & $7.4(0.6-75.4)$ \\
Pathologic Gleason sum & \\
$\leq 6$ & 7 \\
$8-10$ & $138(67.3 \%)$ \\
Low & $25(12.2 \%)$ \\
NCCN Risk Group & $96(46.8 \%)$ \\
Intermediate & $68(33.2 \%)$ \\
High & $40(19.5 \%)$ \\
Extracapsular extension (\%) & $56(27 \%)$ \\
Seminal Vesicle Invasion (\%) & $31(15 \%)$ \\
Positive Surgical Margins (\%) & $125(61 \%)$ \\
Prior ADT, \% & $0(0 \%)$ \\
\hline
\end{tabular}


Table 3. Association of epithelial plasticity biomarker expression with PSA recurrence.

\begin{tabular}{|c|c|c|c|}
\hline $\begin{array}{c}\text { Biomarker } \\
\text { (number evaluable) }\end{array}$ & $\begin{array}{c}\text { Univariate } \\
\text { Hazard Ratio for } \\
\text { PSA recurrence, } \\
95 \% \mathrm{Cl}\end{array}$ & $\begin{array}{c}5 \text { year } \\
\text { Probability of } \\
\text { PSA } \\
\text { recurrence } \\
(\%)\end{array}$ & $\begin{array}{l}\text { p-value } \\
\text { (log-rank) }\end{array}$ \\
\hline $\begin{array}{r}\text { Average Ki-67 }(n=164) \\
\text { Ki67 quartile } 1(n=45) \\
\text { Ki-67 quartile } 2(n=35) \\
\text { Ki-67 quartile } 3(n=43) \\
\text { Ki-67 quartile } 4(n=41)\end{array}$ & $\begin{array}{c}1.09(1.02-1.16) \\
0.67 \\
0.46 \\
0.80 \\
\text { REF }\end{array}$ & $\begin{array}{c}\mathrm{n} / \mathrm{a} \\
33 \% \\
30 \% \\
43 \% \\
56 \%\end{array}$ & $\begin{array}{c}0.0095 \\
0.19\end{array}$ \\
\hline $\begin{array}{r}\text { SNAIL (maximum), } \mathbf{n}=\mathbf{1 8 8} \\
\text { Absent SNAIL }(\mathrm{n}=58) \\
\text { SNAIL } 1+(\mathrm{n}=74) \\
\text { SNAIL } 2-3+(\mathrm{n}=56)\end{array}$ & $\begin{array}{l}1.5 \\
1.4 \\
\text { REF }\end{array}$ & $\begin{array}{l}\mathrm{n} / \mathrm{a} \\
47 \% \\
45 \% \\
31 \%\end{array}$ & 0.34 \\
\hline $\begin{array}{r}\text { ZEB1 (maximum), } n=189 \\
\text { Absent ZEB1 }(n=160) \\
\text { ZEB1 } 1-2+(n=29)\end{array}$ & $\begin{array}{l}0.91 \\
\text { REF }\end{array}$ & $\begin{array}{l}41 \% \\
48 \%\end{array}$ & 0.76 \\
\hline $\begin{array}{r}\text { Vimentin (maximum), } \mathbf{n = 2 0 3} \\
\text { Absent vimentin }(\mathrm{n}=145) \\
\text { Vimentin } 1-2+(\mathrm{n}=58)\end{array}$ & $\begin{array}{c}1.4 \\
\text { REF }\end{array}$ & $\begin{array}{l}43 \% \\
32 \%\end{array}$ & 0.18 \\
\hline $\begin{array}{r}\text { E-cadherin (minimum), } \mathbf{n}=\mathbf{2 0 5} \\
\text { Absent-low E-cadherin }(\mathrm{n}=29) \\
\text { E-cadherin } 2+(\mathrm{n}=176)\end{array}$ & $\begin{array}{l}0.62 \\
\text { REF }\end{array}$ & $\begin{array}{l}24 \% \\
43 \%\end{array}$ & 0.15 \\
\hline $\begin{array}{r}\text { TWIST (maximum), } \mathbf{n = 1 4 8} \\
\text { Absent-low TWIST }(\mathrm{n}=10) \\
\text { TWIST } 2-3+(\mathrm{n}=138)\end{array}$ & $\begin{array}{l}0.64 \\
\text { REF }\end{array}$ & $\begin{array}{l}50 \% \\
40 \%\end{array}$ & 0.30 \\
\hline
\end{tabular}


Figure 1. Association of Ki-67 biomarker expression (average score per subject across TMA) and NCCN clinical risk group with PSA relapse. A. Correlation of Ki67 expression by quartiles with recurrence-free survival (PSA relapse), shown in a Kaplan-Meier survival plot. B. Correlation of NCCN risk groups with PSA relapse, shown in a Kaplan-Meier survival plot. Low risk includes $P S A<10$, Gleason 6 or less, and pT2a or less pathologic stage. Intermediate risk includes PSA 10-20, Gleason 7, or pT2b. High risk includes PSA>20, Gleason 8-10, or stage T2c or higher.

A.

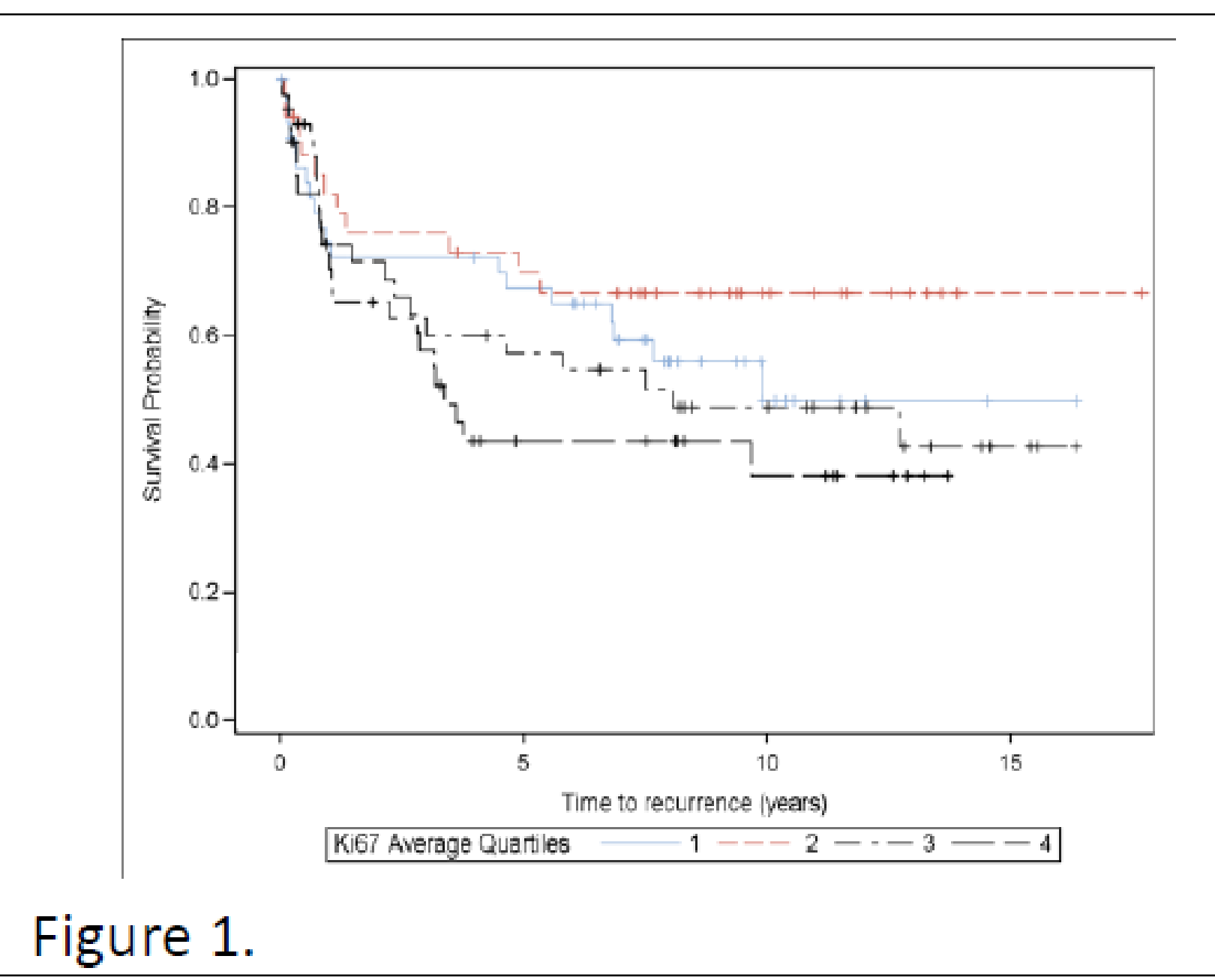


B.

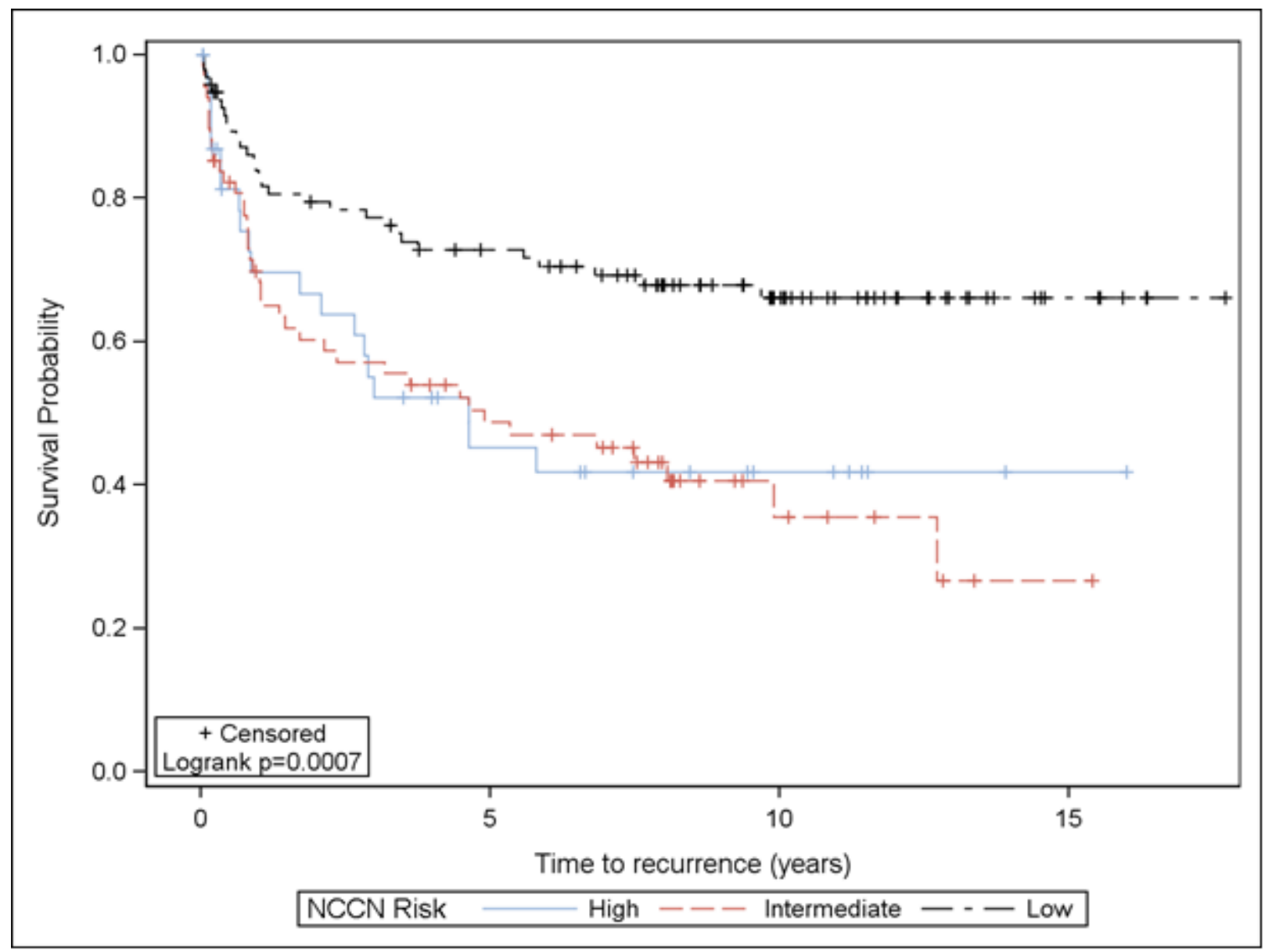




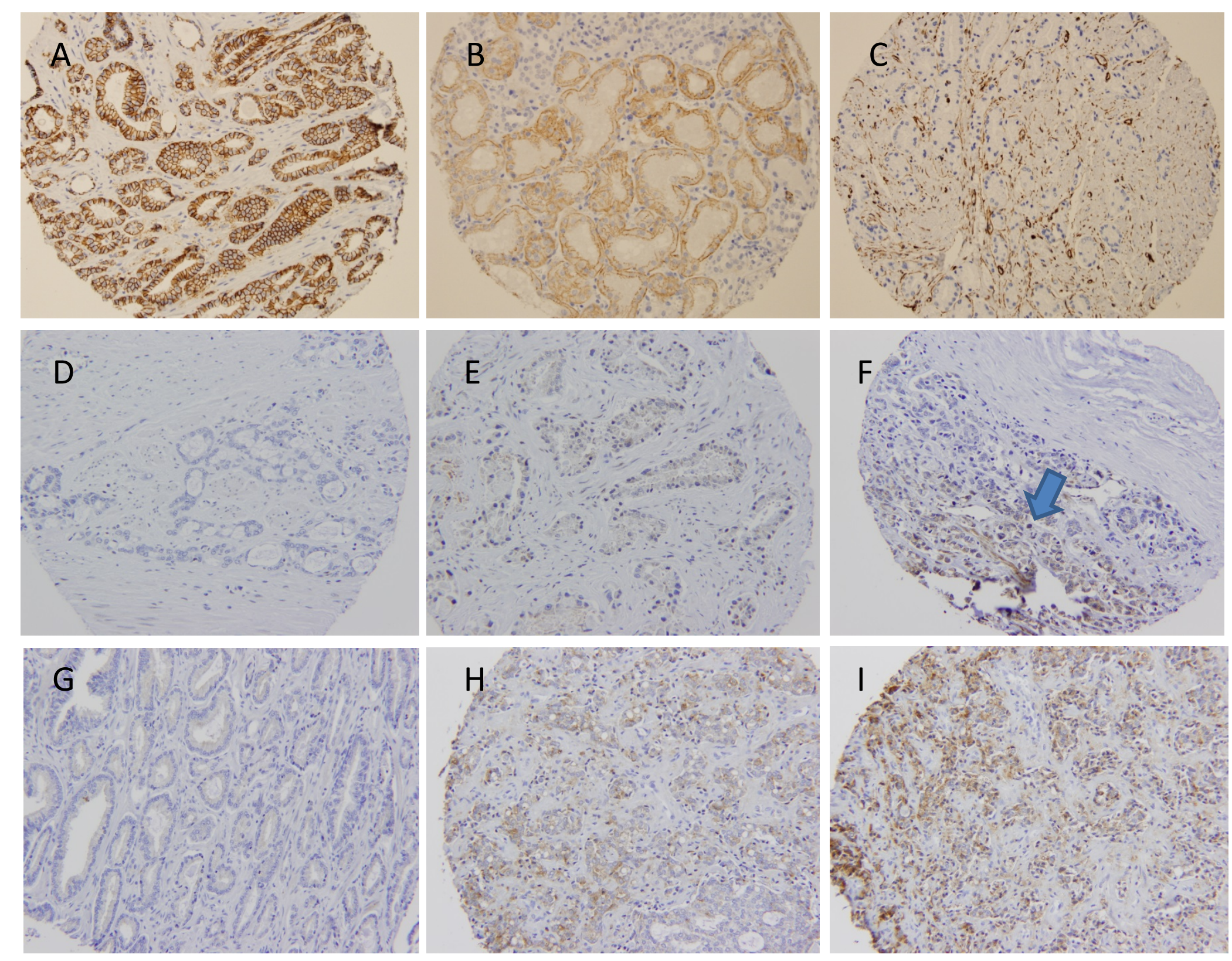

Figure 2. Representative prostate cancer tissue microarray (TMA) immunohistochemical (IHC) staining examples from the Durham VA SEARCH database, stained for A) E-cadherin (2+), B) N-cadherin (1+), and C) vimentin (2+). D-F shows ZEB1 expression (0, 1, and focal 2 (arrow), respectively). G-I shows SNAIL expression $(0,1,2$, respectively). 
Figure 2. Representative prostate cancer tissue microarray (TMA) immunohistochemical $(\mathrm{IHC})$ staining examples from the Durham VA SEARCH database, stained for A) E-cadherin (2+), B) N-cadherin (1+), and C) vimentin (2+). D-F shows ZEB1 expression ( 0,1 , and focal 2 (arrow), respectively). G-I shows SNAIL expression $(0,1,2$, respectively). Images of TWIST expression are available in supplementary figure 1 . 

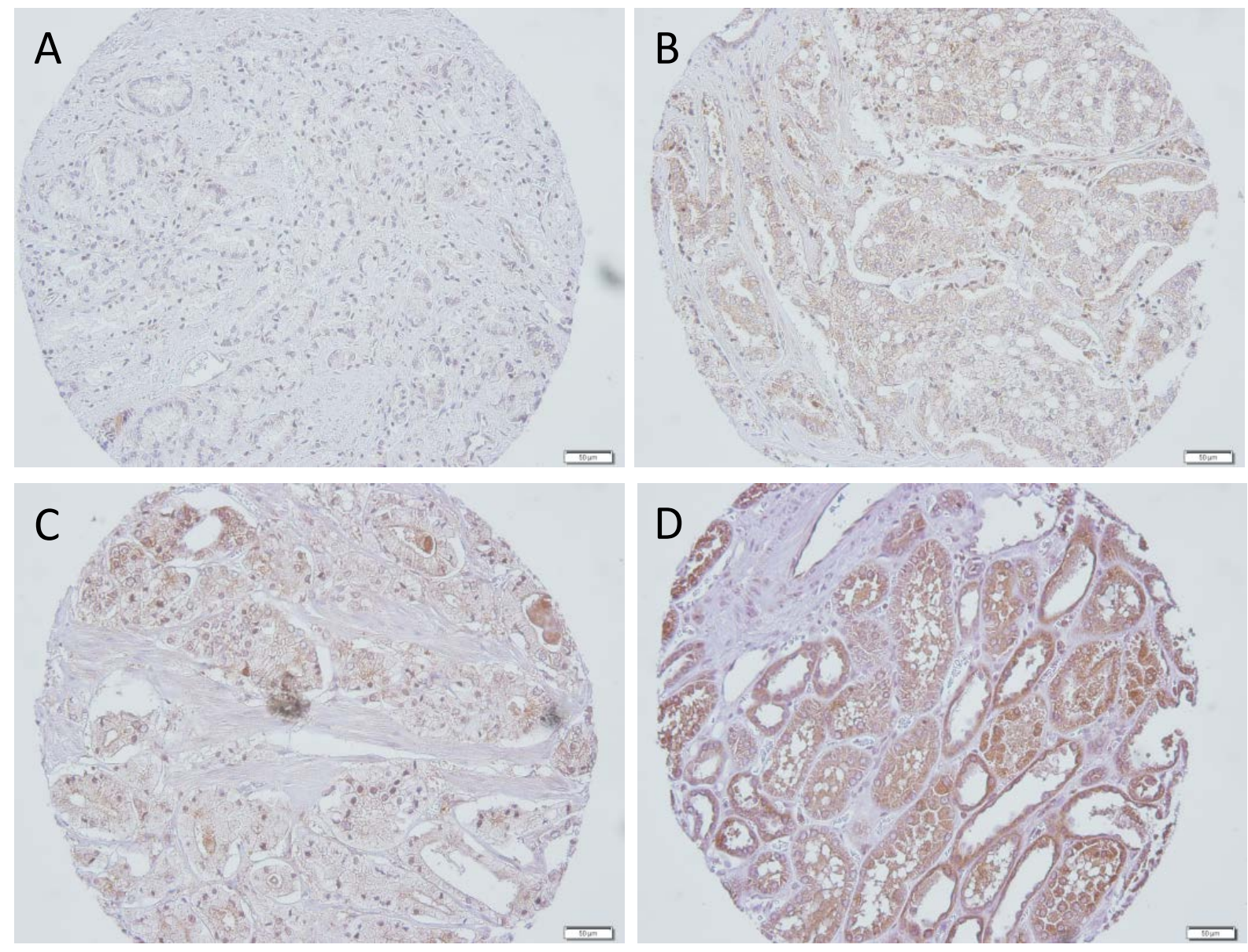

Supplementary Figure 1. Representative prostate cancer tissue microarray (TMA) immunohistochemical (IHC) staining examples from the Durham VA SEARCH database, stained for TWIST, showing absent expression (A), 1+ expression (B), 2+ expression (C), and 3+ expression (D). 


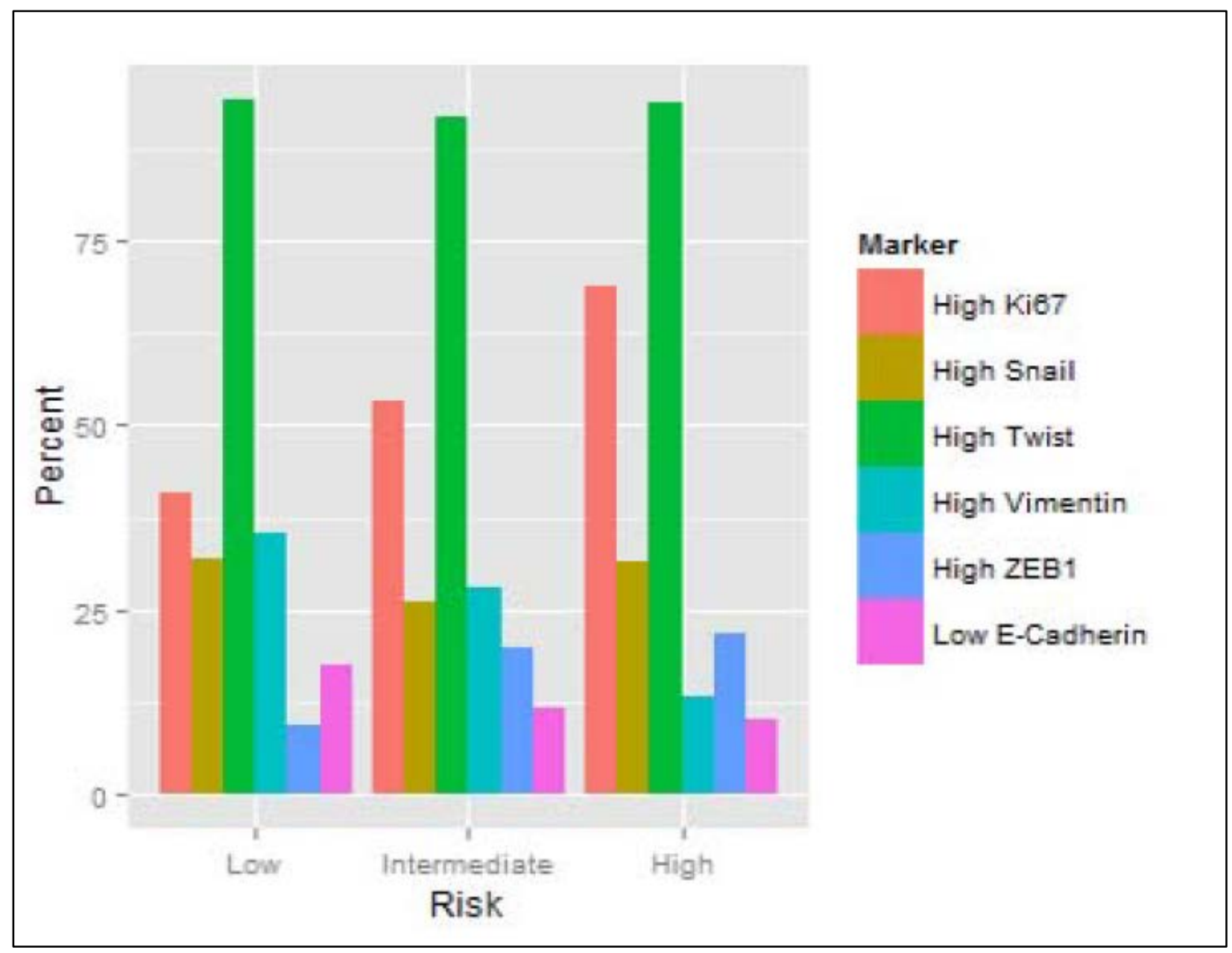

Figure 3. Boxplots demonstrating the association of epithelial plasticity biomarkers with NCCN risk group. Low risk includes $P S A<10$, Gleason 6 or less, and pT2a or less pathologic stage. Intermediate risk includes PSA 10-20, Gleason 7, or pT2b. High risk includes PSA $>20$, Gleason 8-10, or stage T2c or higher. Biomarkers are classified by the proportion high: average Ki67 above median, maximum SNAIL 23+, maximum TWIST 2-3+, maximum vimentin 1-2+, maximum ZEB1 2-3+. For Ecadherin, classification is by the proportion low $(0-1+)$ using the minimum value per subject across the TMA. 


\section{Reference List}

(1) Stephenson AJ, Kattan MW, Eastham JA, et al. Prostate cancer-specific mortality after radical prostatectomy for patients treated in the prostate-specific antigen era. J Clin Oncol 2009;27:4300-5.

(2) D'Amico AV, Whittington R, Malkowicz SB, et al. A multivariate analysis of clinical and pathological factors that predict for prostate specific antigen failure after radical prostatectomy for prostate cancer. J Urol 1995;154:131-8.

(3) Irshad S, Bansal M, Castillo-Martin M, et al. A molecular signature predictive of indolent prostate cancer. Sci Transl Med 2013;5:202ra122.

(4) Bitting RL, Schaeffer D, Somarelli JA, Garcia-Blanco MA, Armstrong AJ. The role of epithelial plasticity in prostate cancer dissemination and treatment resistance. Cancer Metastasis Rev 2014.

(5) Kalluri R, Weinberg RA. The basics of epithelial-mesenchymal transition. J Clin Invest 2009;119:1420-8.

(6) Domingo-Domenech J, Vidal SJ, Rodriguez-Bravo V, et al. Suppression of acquired docetaxel resistance in prostate cancer through depletion of notch- and hedgehog-dependent tumorinitiating cells. Cancer Cell 2012;22:373-88.

(7) Gravdal K, Halvorsen OJ, Haukaas SA, Akslen LA. A switch from E-cadherin to N-cadherin expression indicates epithelial to mesenchymal transition and is of strong and independent importance for the progress of prostate cancer. Clin Cancer Res 2007;13:7003-11.

(8) Tanaka H, Kono E, Tran CP, et al. Monoclonal antibody targeting of $\mathrm{N}$-cadherin inhibits prostate cancer growth, metastasis and castration resistance. Nat Med 2010;16:1414-20.

(9) Armstrong AJ, Marengo MS, Oltean S, et al. Circulating Tumor Cells from Patients with Advanced Prostate and Breast Cancer Display Both Epithelial and Mesenchymal Markers. Mol Cancer Res 2011.

(10) Behnsawy HM, Miyake H, Harada K, Fujisawa M. Expression patterns of epithelial-mesenchymal transition markers in localized prostate cancer: significance in clinicopathological outcomes following radical prostatectomy. BJU Int 2013;111:30-7.

(11) Qin J, Liu X, Laffin B, et al. The PSA(-/lo) prostate cancer cell population harbors self-renewing long-term tumor-propagating cells that resist castration. Cell Stem Cell 2012;10:556-69.

(12) Heeboll S, Borre M, Ottosen PD, Dyrskjot L, Orntoft TF, Torring N. Snail1 is over-expressed in prostate cancer. APMIS 2009;117:196-204.

(13) Ayala G, Tuxhorn JA, Wheeler TM, et al. Reactive stroma as a predictor of biochemical-free recurrence in prostate cancer. Clin Cancer Res 2003;9:4792-801. 
(14) Khor LY, Bae K, Paulus R, et al. MDM2 and Ki-67 predict for distant metastasis and mortality in men treated with radiotherapy and androgen deprivation for prostate cancer: RTOG 92-02. J Clin Oncol 2009;27:3177-84.

(15) Parker AS, Heckman MG, Wu KJ, et al. Evaluation of ki-67 staining levels as an independent biomarker of biochemical recurrence after salvage radiation therapy for prostate cancer. Int J Radiat Oncol Biol Phys 2009;75:1364-70.

(16) Pollack A, DeSilvio M, Khor LY, et al. Ki-67 staining is a strong predictor of distant metastasis and mortality for men with prostate cancer treated with radiotherapy plus androgen deprivation: Radiation Therapy Oncology Group Trial 92-02. J Clin Oncol 2004;22:2133-40.

(17) Verhoven B, Yan Y, Ritter M, et al. Ki-67 is an independent predictor of metastasis and causespecific mortality for prostate cancer patients treated on Radiation Therapy Oncology Group (RTOG) 94-08. Int J Radiat Oncol Biol Phys 2013;86:317-23.

(18) Berney DM, Gopalan A, Kudahetti S, et al. Ki-67 and outcome in clinically localised prostate cancer: analysis of conservatively treated prostate cancer patients from the Trans-Atlantic Prostate Group study. Br J Cancer 2009;100:888-93.

(19) Mak P, Leav I, Pursell B, et al. ERbeta impedes prostate cancer EMT by destabilizing HIF-1alpha and inhibiting VEGF-mediated snail nuclear localization: implications for Gleason grading. Cancer Cell 2010;17:319-32.

(20) Brabletz T, Jung A, Reu S, et al. Variable beta-catenin expression in colorectal cancers indicates tumor progression driven by the tumor environment. Proc Natl Acad Sci U S A 2001;98:1035661.

(21) Haffner MC, Mosbruger T, Esopi DM, et al. Tracking the clonal origin of lethal prostate cancer. J Clin Invest 2013;123:4918-22.

(22) Haffner MC, De Marzo AM, Yegnasubramanian S, Epstein JI, Carter HB. Diagnostic Challenges of Clonal Heterogeneity in Prostate Cancer. J Clin Oncol 2014.

(23) Bitting RL, Boominathan R, Rao C, et al. Development of a method to isolate circulating tumor cells using mesenchymal-based capture. Methods 2013;64:129-36.

(24) Chen CL, Mahalingam D, Osmulski P, et al. Single-cell analysis of circulating tumor cells identifies cumulative expression patterns of EMT-related genes in metastatic prostate cancer. Prostate 2013;73:813-26.

(25) Cottard F, Asmane I, Erdmann E, Bergerat JP, Kurtz JE, Ceraline J. Constitutively active androgen receptor variants upregulate expression of mesenchymal markers in prostate cancer cells. PLoS ONE 2013;8:e63466.

(26) Sun F, Chen HG, Li W, et al. Androgen receptor splice variant AR3 promotes prostate cancer via modulating expression of autocrine/paracrine factors. J Biol Chem 2014;289:1529-39. 
(27) Sun Y, Wang BE, Leong KG, et al. Androgen deprivation causes epithelial-mesenchymal transition in the prostate: implications for androgen-deprivation therapy. Cancer Res 2012;72:527-36. 


\section{Clinical Phenotypes Associated with Circulating Tumor Cell Enumeration in Metastatic Castration Resistant Prostate Cancer}

Rhonda L. Bitting ${ }^{1,4}$, Patrick Healy2 , Susan Halabi ${ }^{2,4}$, Daniel J. George ${ }^{1,3,4}$, Michael Goodin $^{4}$, and Andrew J. Armstrong ${ }^{1,3,4}$

${ }^{1}$ Department of Medicine/Division of Medical Oncology,

${ }^{2}$ Department of Biostatistics and Bioinformatics,

${ }^{3}$ Department of Surgery/Division of Urology,

${ }^{4}$ Duke Cancer Institute, Duke University Medical Center, Durham, NC 27710 USA

Running title: Clinical phenotype, outcomes, and CTC enumeration in men with mCRPC

Keywords: circulating tumor cells, prostate cancer, tumor biomarkers, prognosis, prostate specific antigen

Financial support: Prostate Cancer Foundation Young Investigator Award (AJA) and Department of Defense grant W81XWH-10-1-0483 (AJA)

Correspondence:

Andrew Armstrong, DUMC Box 102002, Durham NC 27710

andrew.armstrong@duke.edu

Conflict of interest: Duke University has a collaborative research agreement with Veridex/Johnson\&Johnson.

Word count: 3,674

Number of figures: 4

Number of tables: 3 


\section{Translational relevance:}

Circulating tumor cells (CTCs) can be enumerated to provide prognostic information in multiple tumor types including men with castration-resistant metastatic prostate cancer (mCRPC). As the genotypic and phenotypic information harbored within CTCs becomes more assessable, it is crucial that we further our understanding of the clinical significance of these cells. Here we further delineate the relationship between CTCs, clinical phenotype, and prognosis in MCRPC. We find that CTC enumeration provides prognostic value independent of other prognostic measures, such as visceral pattern of spread or LDH, hemoglobin, PSA, and alkaline phosphatase levels. We did not identify a poor prognosis, low CTC-producing subset of men with mCRPC. In addition, discordance between PSA and CTC changes during therapy suggest that CTCs may provide additional information on prognosis beyond traditional measures of response, such as imaging or PSA decline. The clinical context is important to consider given the heterogeneity of this disease state. 


\begin{abstract}
Purpose: The presence of $\geq 5$ CTCs is prognostic for shorter survival in men with mCRPC. However, some men have low CTCs despite widespread disease, suggesting heterogeneity in CTC phenotype or detection. The aim of this study was to evaluate the association of CTC enumeration with clinical disease characteristics and overall survival in $\mathrm{mCRPC}$ men at our institution.
\end{abstract}

Experimental Design: CTCs were enumerated using the CellSearch method in a prospective correlative study in mCRPC men starting a new systemic therapy. The primary objective was to determine the clinical phenotype of the subset of poor prognosis mCRPC men that have low CTCs. Secondary endpoints included associations of CTCs with survival and known prognostic biomarkers, prior to therapy and at progression.

Results: At baseline, median CTC count was 16 cells and PSA was $178 \mathrm{ng} / \mathrm{mL}$. At progression, median CTC count was 42, PSA was $245 \mathrm{ng} / \mathrm{mL}, \mathrm{LDH}$ and alkaline phosphatase rose, and hemoglobin dropped. The median OS for this heavily pretreated population was 11.2 months, and the multivariable hazard ratio for death for men with CTCs $<5$ vs. $\geq 5$ was 0.43 (95\% Cl 0.24-0.77). Median PFS was 4.4 months. CTC enumeration modestly correlated with LDH and alkaline phosphatase but only weakly correlated with PSA and hemoglobin. We were unable to identify a consistent subgroup of poor prognosis men with a low number of CTCs.

Conclusion: CTC enumeration appears to be prognostic in men with mCRPC and describes a phenotype of hematogenous dissemination that cannot be predicted based on standard clinical and laboratory assessments. 


\section{BACKGROUND}

Circulating tumor cells (CTCs) are cells that have migrated from primary or metastatic tumor sites and intravasated into the circulation. The presence of $\geq 5$ CTCs using the CellSearch ${ }^{\circledR}$ epithelial cell adhesion molecule (EpCAM)-based ferromagnetic assay is prognostic for shorter survival in men with metastatic castration-resistant prostate cancer (mCRPC) (1), and CTCs can be enumerated to provide prognostic information in multiple other solid tumor types (2-5). However, a substantial number (30-40\%) of men with advanced CRPC have low or undetectable CTCs using the CellSearch ${ }^{\circledR}$ epithelial-based method, despite widespread metastatic disease (6). While these men with low CTCs despite progressive disease have an improved prognosis as compared to those with higher CTCs, they represent a heterogeneous group, and outcomes remain quite poor with median survival estimates of only 1.5 to 3 years.

In women with metastatic breast cancer, patients with aggressive disease phenotypes such as triple-negative histology, inflammatory breast cancer, or brain metastases have fewer detectable CTCs than expected for their burden of disease, despite their poor prognosis (7). This under-detection of CTCs may be due to phenotypic plasticity and the presence of epithelial-mesenchymal transitions (EMT), rendering epithelial-based CTC capture less useful $(8,9)$. Several intrinsic phenotypes of breast cancer lack EpCAM expression, and cells from these tumors go undetected using the standard EpCAM-based assay (10). In many other aggressive metastatic solid tumors such as lung cancer or GI cancers, the under-detection problem may be even more manifest $(4,11,12)$.

There is evidence for phenotypic heterogeneity among CTCs, with some CTCs expressing not only epithelial proteins, but also mesenchymal and stemness proteins, 
indicators of epithelial plasticity (EP) (8). EP refers to the reversible loss of the epithelial cellular phenotype and has been linked to the acquisition of mesenchymal and/or stemness properties and to chemoresistance, invasion, and dissemination in multiple preclinical models of cancer $(13,14)$. In prostate cancer, mesenchymal biomarkers are up-regulated during androgen deprivation therapy (ADT), revert upon replacement of testosterone, and are linked to an increased metastatic propensity and chemotherapy resistance (15-17). Recent studies have suggested links between EP and resistance and progression despite radical prostatectomy, radiation, hormonal therapy, and immunotherapy (reviewed (18)).

Although there are many methods under development to capture CTCs from the blood of cancer patients, the only FDA-cleared technology is CellSearch ${ }^{\circledR}$, which utilizes an anti-EpCAM ferrofluid to capture CTCs and follows with additional staining to visualize the cells and differentiate them from leukocytes. Using CellSearch $®$, CTCs have been shown to be extremely rare in individuals without malignancy and present at a wide range of frequencies in patients with various metastatic carcinomas (11). Due to EP, CTCs with a mesenchymal or transitional phenotype may be missed by CellSearch $\circledast$ and other epithelial-based technologies $(10,19,20)$.

A range of clinical phenotypes exist in men with castration resistant metastatic prostate cancer (reviewed (21)). These phenotypes are determined by the pattern of metastatic spread (visceral, bone, node only), PSA production, anemia, symptoms such as pain and fatigue, levels of bone biomarkers such as alkaline phosphatase, and histology such as adenocarcinoma and neuroendocrine variant tumors. These clinical phenotypes are linked to survival, and are commonly used to determine prognosis in the 
clinic and eligibility/stratification for clinical trials. Several studies have examined the association of clinical phenotype with CTC enumeration, illustrating that CTCs are more common in men with bone metastases, high PSA, and in the post-docetaxel CRPC setting $(1,22)$. These studies have established the independent association of CTC enumeration with survival $(1,3,23)$. However, the systematic examination of the association of clinical phenotype in men with a low versus high CTC count has not been performed.

Here we evaluated the association of CTC enumeration, both prior to a new systemic therapy and at progression on a given systemic therapy, with baseline characteristics and clinical outcomes in men with mCRPC at the Duke Cancer Institute. We hypothesized that men with mCRPC and Gleason 8-10 disease, visceral metastases, low PSA production, or pain would have shorter survival duration but lower CTCs than expected, similar to that reported in triple negative breast cancer patients (7). We speculated that poorly differentiated tumors may have lost their epithelial character, at least in part, and may have CTCs that lack EpCAM expression and are thus under-detected by the CellSearch ${ }^{\circledR}$ assay despite a poor prognosis.

\section{METHODS}

Men with progressive, mCRPC consented and enrolled in 1 of 2 IRB-approved prospective correlative clinical protocols prior to initiating a new systemic therapy. Eligibility for this study included the presence of metastatic disease, progression by PSA, bone scan, or soft tissue/visceral disease criteria (new lesions or progression of existing lesions), and a serum testosterone level of $<50 \mathrm{ng} / \mathrm{dl}$. Men were enrolled prior 
to initiating a new systemic therapy for CRPC, including enzalutamide, docetaxel, abiraterone acetate, cabazitaxel, or novel agents on a separate clinical protocol. Men were excluded if they had received an anthracycline or mitoxantrone within 7 days of blood draw to reduce the risk CTC autofluorescence caused by these agents. All men provided informed consent.

The EpCAM-based CellSearch ${ }^{\circledR}$ platform was used for CTC detection and enumeration, as described previously (11). Results of cell enumeration were expressed as the number of cells per $7.5 \mathrm{~mL}$ of blood. CTC enumeration was performed at baseline in all men prior to the initiation of a new treatment. Men also had blood drawn for CTC enumeration following progression as determined by the treating physician (clinical, radiographic, or PSA progression). Laboratory studies that were collected and measured as part of standard-of-care included baseline hemoglobin (hgb), lactate dehydrogenase (LDH), alkaline phosphatase (AP), liver and kidney function, and PSA. Clinical parameters that were analyzed included pattern of metastatic spread (liver, lung, bone, lymph node metastasis patterns), pain on a visual analog scale (0-10), performance status, prior therapy exposure (docetaxel, hormonal therapies, surgery, radiation), Gleason sum, and demographic data. Outcomes of interest included overall survival and time-to-progression as determined by the treating physician.

The primary endpoint of this prospective analysis was to determine the clinical phenotype of the subset of men with MCRPC and a poor prognosis who have low CTCs $(<5)$ as determined by the standard CellSearch ${ }^{\circledR}$ platform, and to test whether men with high grade tumors, visceral spread, low PSA levels despite metastatic disease, and pain are clinical phenotypes associated with relatively low CTCs. Secondary objectives 
included the association of CTC enumeration at baseline and progression with clinical and laboratory parameters associated with prognosis in men with CRPC. As an exploratory analysis, we also examined the concordance of clinical and PSA progression with CTC progression, hypothesizing that some men with visceral or clinical progression may not have CTC progression due to the presence of low EpCAM status and epithelial plasticity.

\section{Statistical methods}

The association between baseline CTC count and PSA, alkaline phosphatase, lactate dehydrogenase, and hemoglobin was explored using the Spearman correlation (r). The relationship with Gleason sum, race, sites of metastasis, pain score, and prior docetaxel exposure was explored using descriptive statistics. Overall survival (OS) and progression-free survival (PFS) distributions were estimated using the Kaplan-Meier method. OS was calculated from the date of baseline CTC measurement at enrollment to the date of death or date of last follow-up. PFS was calculated from the date of baseline CTC measurement at enrollment to the date of progression or date of death if the patient did not progress. Patients alive who had not progressed as of last follow-up were censored at last follow-up. Hazard ratios and 95\% confidence interval for the HRs were computed using the Cox model. Concordance between (1) CTC and PSA response and (2) CTC and PSA progression was measured using Kendall's tau-b. CTC response was defined as either $<5$ CTCs, which is the standard definition, or as CTC count less than or equal to baseline (if not $<5$ ). PSA response was defined as either a $\geq 30 \%$ decline from baseline or $a \geq 50 \%$ decline from baseline, given that both definitions 
are commonly used and reported in trials of men with mCRPC. Confirmation was not required. CTC progression was defined as any increase from baseline count and as a CTC count $\geq 5$. PSA progression was defined as a PSA of $>25 \%$ above baseline.

\section{RESULTS}

A total of 95 men with mCRPC initially consented to the study, but 6 subjects either withdrew consent or never had baseline data collected and were therefore excluded from analysis, leaving 89 subjects with evaluable baseline data and assessable survival outcomes. Of these, 80 subjects had follow-up laboratory and/or clinical data available, and 70 men have died. At the time of data analysis, 78 of 80 evaluable subjects had disease progression, defined either as radiographic progression $(n=37)$, symptomatic progression or death $(n=21)$, PSA progression $(n=7)$, or both symptomatic and PSA progression $(n=13)$, as per the discretion of the treating physician. CTC count at the time of disease progression was available for 57 of 78 $(73 \%)$ subjects. Median follow-up time for the surviving patients was 10.4 months (95\% Cl: $5.2,15.8)$.

As shown in Table 1, the median age of our metastatic CRPC population was 69 years, and $25 \%$ of the men were African American. $66 \%$ of men had high-grade (Gleason 8-10) tumors. At baseline, the majority of men (92\%) had bony metastases, while $31 \%$ of men had visceral metastases. All men had ongoing androgen deprivation, and $70 \%$ of men had previously received docetaxel chemotherapy. The baseline median CTC count was 16 cells per $7.5 \mathrm{~mL}$ whole blood, with $34 \%$ of subjects having a favorable CTC count of $<5$. There was no difference in baseline CTC count by race. Baseline median PSA was $177.8 \mathrm{ng} / \mathrm{mL}$, baseline median hemoglobin was $11.2 \mathrm{~g} / \mathrm{dL}$, 
and baseline medians for both LDH and alkaline phosphatase were $232.5 \mathrm{U} / \mathrm{L}$ and 132 $\mathrm{U} / \mathrm{L}$, respectively (normal institutional range up to $200 \mathrm{U} / \mathrm{L}$ and $110 \mathrm{U} / \mathrm{L}$, respectively). On a visual analog scale of $0-10,31 \%$ of men had a baseline pain score of 4 or greater, indicating significant pain.

When comparing median CTC enumeration and distributions by clinical phenotype at baseline, men with visceral disease had a median CTC of 19 (IQR 6-55) compared to men with non-visceral bone metastatic disease, who had a median CTC of 16 (IQR 4-54). For men with high-grade poorly differentiated primary tumors (Gleason 8-10), the median CTC was 16 (IQR 3-75) compared to of 7 (IQR 2-44) in men with more well differentiated tumors (Gleason $\leq 7$ ).

After enrollment on study, subjects were either treated with chemotherapy $(n=41)$, hormonal therapy $(n=23)$, or other $(n=16)$, as described below. Chemotherapy was either docetaxel-based therapy $(n=24)$, cabazitaxel-based therapy $(n=10)$, mitoxantrone-based therapy $(n=5)$, or other $(n=2)$. Hormonal therapy included either abiraterone $(n=8)$, enzalutamide $(n=7)$, nilutamide $(n=6)$, or ketoconazole $(n=2)$. The remainder of the men received protocol-directed therapies including everolimus $(n=4)$, dasatinib $(n=4)$, sunitinib $(n=1)$, BKM120 $(n=1)$, MLN9708 $(n=1)$, blinded to mitoxantrone or cabazitaxel $(n=1)$, placebo $(n=2)$, or were not treated $(n=2)$.

The associations between CTC count and PSA, AP, LDH, and hgb both at baseline and at progression were explored using the Spearman correlation $(r)$ and are shown in Figure 1. At disease progression, the median CTC count was 42 and PSA was $245 \mathrm{ng} / \mathrm{mL}$. Median LDH and alkaline phosphatase rose slightly, while median hemoglobin dropped to $10.5 \mathrm{~g} / \mathrm{dL}$. At baseline, $\mathrm{LDH}(\mathrm{r}=0.5,95 \% \mathrm{Cl}: 0.26,0.62)$ and 
alkaline phosphatase $(r=.48,95 \% \mathrm{Cl}: 0.24,0.59)$ were significantly positively correlated with CTC count, while the PSA was only weakly correlated $(r=0.2,95 \% \mathrm{Cl}: 0.06,0.45)$. At progression, the correlation between CTC count and PSA level was slightly improved $(r=0.36,95 \% \mathrm{Cl}: 0.11,0.57)$. Hemoglobin at progression was also more strongly negatively correlated with CTC count compared to baseline $(-0.45$ versus -0.14$)$. The median CTC count in men with a baseline pain score $\geq 4$ was 23 compared with 15 in men with a pain score <4. At progression, median CTC count was 96 for men with pain score $\geq 4$ versus 12.5 if pain score $<4$. We found no difference in CTC enumeration at baseline or at progression among men who experienced radiographic progression ( $\mathrm{n}=37$, median 12 CTCs at baseline and 29 CTCs at progression) as compared to men with PSA or clinical progression and no radiographic progression $(n=38$, median 23 CTCs at baseline and 45 CTCs at progression).

As illustrated in Figure 2, the median overall survival for this heavily pre-treated study population was 11.2 months (95\% Cl: $9.2,13.2)$, and the probability of survival at 2 years was $7.3 \%(95 \% \mathrm{Cl}: 2.1,16.8)$. Of these men, $63 \%$ died within one year of study consent, and of those, $79 \%$ had a high CTC count (defined as CTCs $\geq 5$ ) at baseline. When stratified by CTC count, the median OS for patients with a low CTC count (defined as CTCs <5) was 16.6 months $(95 \% \mathrm{Cl}: 11.7,20.9)$ and 8.9 months $(95 \% \mathrm{Cl}$ : 6.3, 11.2) for those with a high CTC count. The univariate HR for OS for men with low vs. high CTCs was $0.41(95 \% \mathrm{Cl} 0.24-0.69)$. As shown in Table 2, in a multivariable model for OS, including the variables of pain, PSA levels, Gleason score, CTC count and visceral metastases, both low CTC count (HR $0.43 ; 95 \% \mathrm{Cl} 0.24,0.77)$ and the lack 
of visceral metastasis ( $\mathrm{HR} 0.44 ; 95 \% \mathrm{Cl} 0.25,0.78$ ) were prognostic for improved survival.

As an exploratory analysis, stratifying by the presence or absence of visceral metastases at baseline, we found that subjects with visceral metastases had a poorer prognosis than those with bone metastases $(\mathrm{HR}=2.11,95 \% \mathrm{Cl}: 1.25,3.57)$. In our dataset and shown in Figure 3, patients with visceral metastases and low CTC counts $(n=7)$ survived longer than patients with visceral metastases and high CTC counts $(n=21)$ (median OS 23.7 vs. 5.6 months, respectively; HR $0.17,95 \% \mathrm{Cl}: 0.04,0.74)$, suggesting that CTC enumeration may further stratify outcomes even in men with a visceral pattern of spread. For men with bone metastasis and no visceral disease, median OS in men with low vs. high CTCs was 16.6 vs 11.0 months $(\mathrm{HR} 0.57,95 \% \mathrm{Cl}$ : $0.31,1.05)$

Analysis of survival by change in CTC count from baseline to progression demonstrates that men who maintain favorable CTC counts $(<5)$ live longer than those with consistently unfavorable counts $(\geq 5)$, with a median OS 27.3 months $(95 \% \mathrm{Cl} 1.8$, $\mathrm{NR})$ versus 9.2 months $(95 \% \mathrm{Cl} 6.3,11.2)$.

The median progression-free survival for this study was 4.4 months, with the Kaplan-Meier curve for PFS by baseline CTC count shown in Figure 4 . Men with baseline CTC count $\geq 5$ had a composite PFS of 3.7 months $(95 \% \mathrm{Cl} 2.5,5.1)$ and those with CTC count $<5$ had a PFS of 5.7 months $(95 \% \mathrm{Cl} 3.6,8.0)$.

Of the subjects evaluable for disease progression, 14 men $(18 \%)$ had $<5$ CTCs at the time of progression. We found no significant differences in age, prevalence of high Gleason tumors, or visceral disease among men who progressed with low CTCs 
vs. those men who progressed with high CTCs. The median time to progression for these men was 6.2 months $(95 \% \mathrm{Cl} 2.1,9.6)$ and the median overall survival was 22 months. Only 4 men had an unfavorable to favorable CTC conversion despite clear evidence of disease progression (death $n=1$, radiographic progression $n=1$, symptoms $n=2)$.

Finally, we analyzed the relationship between PSA changes and CTC changes during treatment response and progression. To determine response concordance for PSA and CTC enumeration, we explored a PSA response of both $\geq 30 \%$ and $\geq 50 \%$ decline from baseline, and CTC response was defined as a count of $<5$ or less than baseline CTC count. To evaluate concordance at progression, PSA progression was defined as $>25 \%$ above baseline, and CTC progression was defined as a count above baseline or $\geq 5$. In all cases, the Kendall's Tau was between $0.28(95 \% \mathrm{Cl}: 0.11,0.58)$ and $0.34(95 \% \mathrm{Cl}: 0.03,0.52)$, indicating only moderate concordance between CTC and PSA responses (with perfect concordance represented by a tau of 1). The results are shown in Table 3, and due to similar results regardless of definition, we show PSA response defined as $\geq 30 \%$ decline from baseline and CTC progression defined as a count $\geq 5$. Patients with both a PSA and CTC response $(n=10)$ had a median OS of 18.5 months. Patients with neither a PSA nor CTC response $(n=28)$ had a median OS of 10 months. Men with either a PSA response $(n=3)$ or CTC response $(n=16)$ alone did not survive as long as did men with a response defined by both biomarkers. Assessment of PSA and CTC progression concordance yielded similar results (Kendall's Tau of 0.280.29). Patients with both PSA and CTC progression $(n=26)$ had a median OS of 6.8 
months, but men with either PSA or CTC progression alone survived longer (12.2-13.2 months). Men without evidence of PSA or CTC progression ( $n=10)$ lived 22.1 months.

\section{DISCUSSION}

The independent prognostic value of CTC enumeration in men with MCRPC is well established, using the CellSearch ${ }^{\circledR}$ platform and a threshold of 5 or more cells (1). Furthermore, during treatment with docetaxel chemotherapy, a change in CTC count from $\geq 5$ to less than 5 is associated with an improved prognosis as compared with CTC progression or lack of response $(3,23)$. Our study reports on several important novel findings, despite the relatively small sample size and exploratory nature of the analysis. One is that CTC enumeration is only moderately correlated with other known prognostic biomarkers (PSA, hemoglobin, $\mathrm{LDH}, \mathrm{AP}$ ), and remains an important independent prognostic biomarker even in men with visceral or bone metastatic prostate cancer phenotypes. This indicates that epithelial CTC production in some men with CRPC, as determined by the EpCAM-based CellSearch ${ }^{\circledR}$ platform, is often uncoupled to AR activity and PSA levels, bone biomarkers, and tumor metabolism biomarkers such as LDH. In our study, men with visceral metastases had a poorer prognosis than those with bone metastases, consistent with other published data (24). Furthermore, we were able to additionally stratify patient outcomes by evaluating both the site of metastasis and the CTC count.

We were unable to identify a subgroup of men with high-grade or visceral CRPC and a low CTC count despite a poor prognosis, further supporting the importance of measuring CTC in the setting of poor prognostic clinical phenotypes. Our data suggests that a high CTC count in combination with visceral metastasis portends a very poor 
prognosis, and if validated in larger data sets, this finding could help differentiate prognosis within clinical phenotypes. This result may, however, be due to our limited sample size and reduced power to identify such subgroup populations, and it remains possible that additional CTCs that lack EpCAM or epithelial biomarkers may exist in men with mCRPC, as demonstrated by our prior work (8). However, we were not able to identify a consistent subgroup of men with very low CTCs that have a poorer prognosis. Overall, men with visceral metastatic CRPC had similar median CTC counts to men with non-visceral metastatic CRPC, despite a much worse prognosis, which may indicate that these men with visceral disease have additional undetected CTCs. Further studies using novel CTC capture approaches will be needed to further delineate this biology.

Our study suggests that, even at the time of disease progression, having a low CTC count remains a good prognostic indicator. Despite evidence that CTCs lose their epithelial phenotype during disease progression (25), our study suggests that epithelial CTCs remain relevant and inform upon prognosis. When followed longitudinally, the CTC count can be used to update a man's prognosis, which appears to be better when few or zero CTCs are detected. This is consistent with recently published data showing that a rising СTC count after 3 weeks of treatment on docetaxel portends a worse prognosis (23).

Another major finding of our analysis is the discordance between CTC enumeration and PSA change at response and progression. In our study, it was not possible to predict CTC response based on PSA declines, nor vice versa, given the modest associations of PSA and CTC response with each other. Our results would 
support the hypothesis that knowledge of both CTC and PSA levels over time, using several definitions of response or progression, provides additional prognostic information beyond that determined by either test alone. Having both CTC and PSA progression may be associated with a worse survival, relative to progression by either CTC count or PSA level alone. While CTC and PSA levels themselves do not determine the optimal systemic management of men with mCRPC, knowledge of a composite of CRPC biomarkers including CTCs, PSA, bone biomarkers, imaging results, and patient symptoms may ensure that all information is considered in determining whether to continue an ongoing therapy or change to an alternative therapeutic strategy.

The limitations of our study include the overall small sample size, which restricts our ability to conduct extensive multivariable analyses and reduces the power to make associations of CTC enumeration with more subtle clinical phenotypes. Despite this, however, we observed an independent prognostic role for CTC enumeration over time and a modest correlation of CTCs with other known prognostic biomarkers, suggesting that hematogenous dissemination is an intrinsic property of aggressive and lethal prostate cancer that is not fully captured by PSA levels, bone turnover biomarkers, and imaging.

In summary, CTC enumeration provides prognostic information that is distinct from other clinical phenotypic data such as patterns of metastatic spread, PSA levels, bone turnover biomarkers, and tumor grade. CTC enumeration may further stratify outcomes in men with $\mathrm{mCRPC}$ and visceral or bone metastasis. A poor prognostic subgroup of CRPC men with $<5$ CTCs as measured by the CellSearch ${ }^{\circledR}$ method could 
not be reliably identified. However, some men did have low CTCs despite widespread metastatic disease, likely reflecting heterogeneity in CTCs, some of which may be dissociated in part from epithelial and androgen receptor activity (as reflected by loss of EpCAM and discordance with PSA values). We hypothesize that CTC burden may be also associated with bone microenvironmental factors, hypoxia, and tumor burden (as reflected by AP and LDH values). Further prospective and controlled studies using novel CTC capture and isolation methods will be needed to test these findings. In addition, further clinical studies are needed to evaluate if modifying therapy based on CTC changes over time can result in improved clinical outcomes. 


\section{REFERENCES}

1. Danila DC, Heller G, Gignac GA, Gonzalez-Espinoza R, Anand A, Tanaka E, et al. Circulating tumor cell number and prognosis in progressive castration-resistant prostate cancer. Clinical cancer research : an official journal of the American Association for Cancer Research. 2007;13(23):7053-8. Epub 2007/12/07. doi: 10.1158/1078-0432.CCR-07-1506. PubMed PMID: 18056182.

2. Cristofanilli M, Budd GT, Ellis MJ, Stopeck A, Matera J, Miller MC, et al. Circulating tumor cells, disease progression, and survival in metastatic breast cancer. The New England journal of medicine. 2004;351(8):781-91. Epub 2004/08/20. doi: 10.1056/NEJMoa040766. PubMed PMID: 15317891.

3. de Bono JS, Scher HI, Montgomery RB, Parker C, Miller MC, Tissing H, et al. Circulating tumor cells predict survival benefit from treatment in metastatic castration-resistant prostate cancer. Clinical cancer research : an official journal of the American Association for Cancer Research. 2008;14(19):63029. Epub 2008/10/03. doi: 10.1158/1078-0432.CCR-08-0872. PubMed PMID: 18829513.

4. Cohen SJ, Punt CJ, lannotti N, Saidman BH, Sabbath KD, Gabrail NY, et al. Relationship of circulating tumor cells to tumor response, progression-free survival, and overall survival in patients with metastatic colorectal cancer. Journal of clinical oncology : official journal of the American Society of Clinical Oncology. 2008;26(19):3213-21. Epub 2008/07/02. doi: 10.1200/JCO.2007.15.8923. PubMed PMID: 18591556.

5. Khan MS, Kirkwood A, Tsigani T, Garcia-Hernandez J, Hartley JA, Caplin ME, et al. Circulating tumor cells as prognostic markers in neuroendocrine tumors. Journal of clinical oncology : official journal of the American Society of Clinical Oncology. 2013;31(3):365-72. Epub 2012/12/19. doi: 10.1200/JCO.2012.44.2905. PubMed PMID: 23248251.

6. Scher HI, Morris MJ, Basch E, Heller G. End points and outcomes in castration-resistant prostate cancer: from clinical trials to clinical practice. Journal of clinical oncology : official journal of the American Society of Clinical Oncology. 2011;29(27):3695-704. Epub 2011/08/24. doi: 10.1200/JCO.2011.35.8648. PubMed PMID: 21859988.

7. Mego M, De Giorgi U, Dawood S, Wang XM, Valero V, Andreopoulou E, et al. Characterization of metastatic breast cancer patients with nondetectable circulating tumor cells. Int J Cancer. 2011;129(2):417-23. doi: Doi 10.1002/ljc.25690. PubMed PMID: WOS:000291603900015.

8. Armstrong AJ, Marengo MS, Oltean S, Kemeny G, Bitting RL, Turnbull JD, et al. Circulating tumor cells from patients with advanced prostate and breast cancer display both epithelial and mesenchymal markers. Molecular cancer research : MCR. 2011;9(8):997-1007. Epub 2011/06/15. doi: 10.1158/15417786.MCR-10-0490. PubMed PMID: 21665936; PubMed Central PMCID: PMC3157566.

9. Yu M, Bardia A, Wittner BS, Stott SL, Smas ME, Ting DT, et al. Circulating breast tumor cells exhibit dynamic changes in epithelial and mesenchymal composition. Science. 2013;339(6119):580-4. Epub 2013/02/02. doi: 10.1126/science.1228522. PubMed PMID: 23372014.

10. Sieuwerts AM, Kraan J, Bolt J, van der Spoel P, Elstrodt F, Schutte M, et al. Anti-epithelial cell adhesion molecule antibodies and the detection of circulating normal-like breast tumor cells. Journal of the National Cancer Institute. 2009;101(1):61-6. Epub 2009/01/01. doi: 10.1093/jnci/djn419. PubMed PMID: 19116383; PubMed Central PMCID: PMC2639293.

11. Allard WJ, Matera J, Miller MC, Repollet M, Connelly MC, Rao C, et al. Tumor cells circulate in the peripheral blood of all major carcinomas but not in healthy subjects or patients with nonmalignant diseases. Clinical cancer research : an official journal of the American Association for Cancer Research. 2004;10(20):6897-904. Epub 2004/10/27. doi: 10.1158/1078-0432.CCR-04-0378. PubMed PMID: 15501967.

12. Krebs MG, Hou JM, Sloane R, Lancashire L, Priest L, Nonaka D, et al. Analysis of circulating tumor cells in patients with non-small cell lung cancer using epithelial marker-dependent and -independent approaches. Journal of thoracic oncology : official publication of the International Association for the 
Study of Lung Cancer. 2012;7(2):306-15. Epub 2011/12/17. doi: 10.1097/JTO.0b013e31823c5c16. PubMed PMID: 22173704.

13. Mani SA, Guo W, Liao MJ, Eaton EN, Ayyanan A, Zhou AY, et al. The epithelial-mesenchymal transition generates cells with properties of stem cells. Cell. 2008;133(4):704-15. Epub 2008/05/20. doi: 10.1016/j.cell.2008.03.027. PubMed PMID: 18485877; PubMed Central PMCID: PMC2728032.

14. Kong D, Banerjee S, Ahmad A, Li Y, Wang Z, Sethi S, et al. Epithelial to mesenchymal transition is mechanistically linked with stem cell signatures in prostate cancer cells. PloS one. 2010;5(8):e12445. Epub 2010/09/02. doi: 10.1371/journal.pone.0012445. PubMed PMID: 20805998; PubMed Central PMCID: PMC2929211.

15. Sun Y, Wang BE, Leong KG, Yue P, Li L, Jhunjhunwala S, et al. Androgen deprivation causes epithelial-mesenchymal transition in the prostate: implications for androgen-deprivation therapy. Cancer research. 2012;72(2):527-36. Epub 2011/11/24. doi: 10.1158/0008-5472.CAN-11-3004. PubMed PMID: 22108827.

16. Domingo-Domenech J, Vidal SJ, Rodriguez-Bravo V, Castillo-Martin M, Quinn SA, RodriguezBarrueco R, et al. Suppression of Acquired Docetaxel Resistance in Prostate Cancer through Depletion of Notch- and Hedgehog-Dependent Tumor-Initiating Cells. Cancer cell. 2012;22(3):373-88. Epub 2012/09/15. doi: 10.1016/j.ccr.2012.07.016. PubMed PMID: 22975379.

17. Tanaka H, Kono E, Tran CP, Miyazaki H, Yamashiro J, Shimomura T, et al. Monoclonal antibody targeting of $\mathrm{N}$-cadherin inhibits prostate cancer growth, metastasis and castration resistance. Nature medicine. 2010;16(12):1414-20. Epub 2010/11/09. doi: 10.1038/nm.2236. PubMed PMID: 21057494; PubMed Central PMCID: PMC3088104.

18. Bitting RL, Schaeffer D, Somarelli JA, Garcia-Blanco MA, Armstrong AJ. The Role of Epithelial Plasticity in Prostate Cancer Dissemination and Treatment Resistance. Cancer and Metastasis Reviews. 2014. doi: 10.1007/s10555-013-9483-z.

19. Aktas B, Tewes M, Fehm T, Hauch S, Kimmig R, Kasimir-Bauer S. Stem cell and epithelialmesenchymal transition markers are frequently overexpressed in circulating tumor cells of metastatic breast cancer patients. Breast cancer research : BCR. 2009;11(4):R46. Epub 2009/07/11. doi: 10.1186/bcr2333. PubMed PMID: 19589136; PubMed Central PMCID: PMC2750105.

20. Raimondi C, Gradilone A, Naso G, Vincenzi B, Petracca A, Nicolazzo C, et al. Epithelialmesenchymal transition and stemness features in circulating tumor cells from breast cancer patients. Breast cancer research and treatment. 2011;130(2):449-55. Epub 2011/02/08. doi: 10.1007/s10549-0111373-x. PubMed PMID: 21298334.

21. Zhang T, Armstrong AJ. Clinical phenotypes of castration-resistant prostate cancer. Clinical advances in hematology \& oncology : H\&O. 2013.

22. Amato RJ, Melnikova V, Zhang Y, Liu W, Saxena S, Shah PK, et al. Epithelial cell adhesion molecule-positive circulating tumor cells as predictive biomarker in patients with prostate cancer. Urology. 2013;81(6):1303-7. Epub 2013/04/30. doi: 10.1016/j.urology.2012.10.041. PubMed PMID: 23622774.

23. Goldkorn A, Ely B, Quinn DI, Tangen CM, Fink LM, Xu T, et al. Circulating Tumor Cell Counts Are Prognostic of Overall Survival in SWOG S0421: A Phase III Trial of Docetaxel With or Without Atrasentan for Metastatic Castration-Resistant Prostate Cancer. Journal of clinical oncology : official journal of the American Society of Clinical Oncology. 2014. Epub 2014/03/13. doi: 10.1200/JCO.2013.51.7417. PubMed PMID: 24616308.

24. Pond GR, Sonpavde G, de Wit R, Eisenberger MA, Tannock IF, Armstrong AJ. The Prognostic Importance of Metastatic Site in Men with Metastatic Castration-resistant Prostate Cancer. European urology. 2014;65(1):3-6. Epub 2013/10/15. doi: 10.1016/j.eururo.2013.09.024. PubMed PMID: 24120464. 
25. Chen CL, Mahalingam D, Osmulski P, Jadhav RR, Wang CM, Leach RJ, et al. Single-cell analysis of circulating tumor cells identifies cumulative expression patterns of EMT-related genes in metastatic prostate cancer. The Prostate. 2013;73(8):813-26. Epub 2013/01/03. doi: 10.1002/pros.22625. PubMed PMID: 23280481. 


\begin{tabular}{|c|c|c|}
\hline TABLE 1. BASELINE CHARACTERISTICS & Results $(n=89)$ & \\
\hline Age in years, median (range) & $69(42-94)$ & \\
\hline $\begin{array}{l}\text { Race } \\
\text { White, n (\%) } \\
\text { Black, n (\%) }\end{array}$ & $\begin{array}{l}67(75 \%) \\
22(25 \%)\end{array}$ & \\
\hline $\begin{array}{l}\text { Gleason score, } \mathrm{n}(\%) \\
5 \\
6 \\
7 \\
8 \\
9 \\
10\end{array}$ & $\begin{array}{l}3(4 \%) \\
4(5 \%) \\
21(25 \%) \\
21(25 \%) \\
31(38 \%) \\
3(4 \%)\end{array}$ & \\
\hline $\begin{array}{l}\text { Sites of metastasis } \\
\text { Any bone, } \mathrm{n}(\%) \\
\text { Bone or lymph node/soft tissue, } \mathrm{n}(\%) \\
\text { Any visceral, } \mathrm{n}(\%) \\
\text { Lymph node/soft tissue only, } \mathrm{n}(\%)\end{array}$ & $\begin{array}{l}82(92 \%) \\
59(66 \%) \\
28(31 \%) \\
2(2 \%)\end{array}$ & \\
\hline $\begin{array}{l}\text { Prior therapies } \\
\text { ADT or orchiectomy } \\
\text { Bicalutamide, nilutamide, or flutamide, n (\%) } \\
\text { Ketoconazole, n (\%) } \\
\text { Abiraterone, enzalutamide, or TAK700, n (\%) } \\
\text { Sipuleucel-T, n (\%) } \\
\text { Docetaxel, n (\%) } \\
\text { Cabazitaxel, n (\%) } \\
\text { Other }\end{array}$ & $\begin{array}{l}89(100 \%) \\
79(89 \%) \\
29(33 \%) \\
19(21 \%) \\
14(16 \%) \\
62(70 \%) \\
6(7 \%) \\
0\end{array}$ & $\begin{array}{l}\text { On study therapy } \\
89(100 \%) \\
6(7.5 \%) \\
2(2.5 \%) \\
15(18.6 \%) \\
0 \\
24(30 \%) \\
10(12.5 \%) \\
23(28.8 \%)\end{array}$ \\
\hline LABORATORY VALUES & $\begin{array}{c}\text { BASELINE } \\
(n=89)\end{array}$ & $\begin{array}{c}\text { AT PROGRESSION } \\
(n=75)\end{array}$ \\
\hline $\begin{array}{l}\text { Circulating tumor cells, per } 7.5 \mathrm{~mL} \text { blood } \\
\text { CTC count, median (range) } \\
\text { CTC count }<5, \mathrm{n}(\%) \\
\text { CTC count }<1, \mathrm{n}(\%)\end{array}$ & $\begin{array}{l}n=89 \\
16(0-1014) \\
30(34 \%) \\
10(11 \%)\end{array}$ & $\begin{array}{l}n=57 \\
42(0-1051) \\
14(25 \%) \\
4(7 \%)\end{array}$ \\
\hline $\begin{array}{l}\text { PSA ng/mL, median (range) } \\
\text { LDH U/L, median (range) } \\
\text { Alkaline phosphate U/L, median (range) } \\
\text { Hemoglobin g/dL, median (range) }\end{array}$ & $\begin{array}{l}177.8(6.8-13420) \\
232.5(147-1745) \\
132(36-1308) \\
11.2(7.3-14.9) \\
\end{array}$ & $\begin{array}{l}245(5.7-5842) \\
249.5(141-3170) \\
135(35-3075) \\
10.5(5-13.1) \\
\end{array}$ \\
\hline Pain score $\geq 4, \mathrm{n}(\%)$ & $28(31 \%)$ & $26(35 \%)$ \\
\hline
\end{tabular}

Table 1. Characteristics of patients with metastatic CRPC on this study at baseline (column 1) and characteristics of patients on study at progression (column 2). 
Table 2. Multivariable association of CTC enumeration and other clinical and laboratory biomarkers with survival (OS)*

\begin{tabular}{|l|c|c|}
\hline \multicolumn{1}{|c|}{ Parameter } & Hazard Ratio & $\begin{array}{c}\text { 95\% Confidence } \\
\text { Interval }\end{array}$ \\
\hline CTC (<5 vs. $\geq 5)$ & 0.43 & $0.24,0.77$ \\
Visceral Metastasis (No vs. Yes) & 0.44 & $0.25,0.78$ \\
Pain Score (0 vs. >0) & 0.96 & $0.57,1.59$ \\
Gleason Score (8-10 vs. <8) & 1.22 & $0.71,2.09$ \\
PSA & 1.00 & $1.00,1.00$ \\
\hline
\end{tabular}

*82 patients included in analysis. 
Table 3. Associations between PSA and CTC response and progression.

A. Response concordance. Frequencies, $\mathrm{n}(\%)$, are shown for each condition. CTC response is defined as CTC count $<5$ or less than baseline. PSA response is defined as $\geq 30 \%$ PSA decline from baseline.

\begin{tabular}{|l|c|c|}
\hline $\begin{array}{c}\text { Response concordance } \\
\text { Kendall's Tau-b: } 0.34 \\
95 \% \text { Cl: } 0.11,0.58\end{array}$ & No CTC response & CTC response \\
\hline No PSA response & $28(49.1 \%)$ & $16(28.1 \%)$ \\
\hline PSA response & $3(5.3 \%)$ & $10(17.5 \%)$ \\
\hline
\end{tabular}

B. Progression concordance. Frequencies, $n(\%)$, are shown for each condition. PSA progression is defined as $>25 \%$ above baseline. CTC progression is defined as CTC count $\geq 5$.

\begin{tabular}{|l|c|c|}
\hline $\begin{array}{c}\text { Progression concordance } \\
\text { Kendall's Tau-b }=0.28 \\
95 \% \mathrm{Cl}: 0.03,0.52\end{array}$ & No PSA progression & PSA progression \\
\hline No CTC progression & $10(17.5 \%)$ & $17(29.8 \%)$ \\
\hline CTC progression & $4(7.0 \%)$ & $26(45.6 \%)$ \\
\hline
\end{tabular}


Figure 1. Spearman correlation plots for CTC count and prostate specific antigen (PSA), alkaline phosphatase (AP), lactate dehydrogenase (LDH), and hemoglobin $(\mathrm{Hgb})$, both at baseline and at progression.

Figure 2. Kaplan-Meier plot for overall survival $(n=89)$

Figure 3. Kaplan-Meier Plot for OS by baseline CTC count and site of metastasis Figure 4. Progression free survival stratified by baseline CTC count 

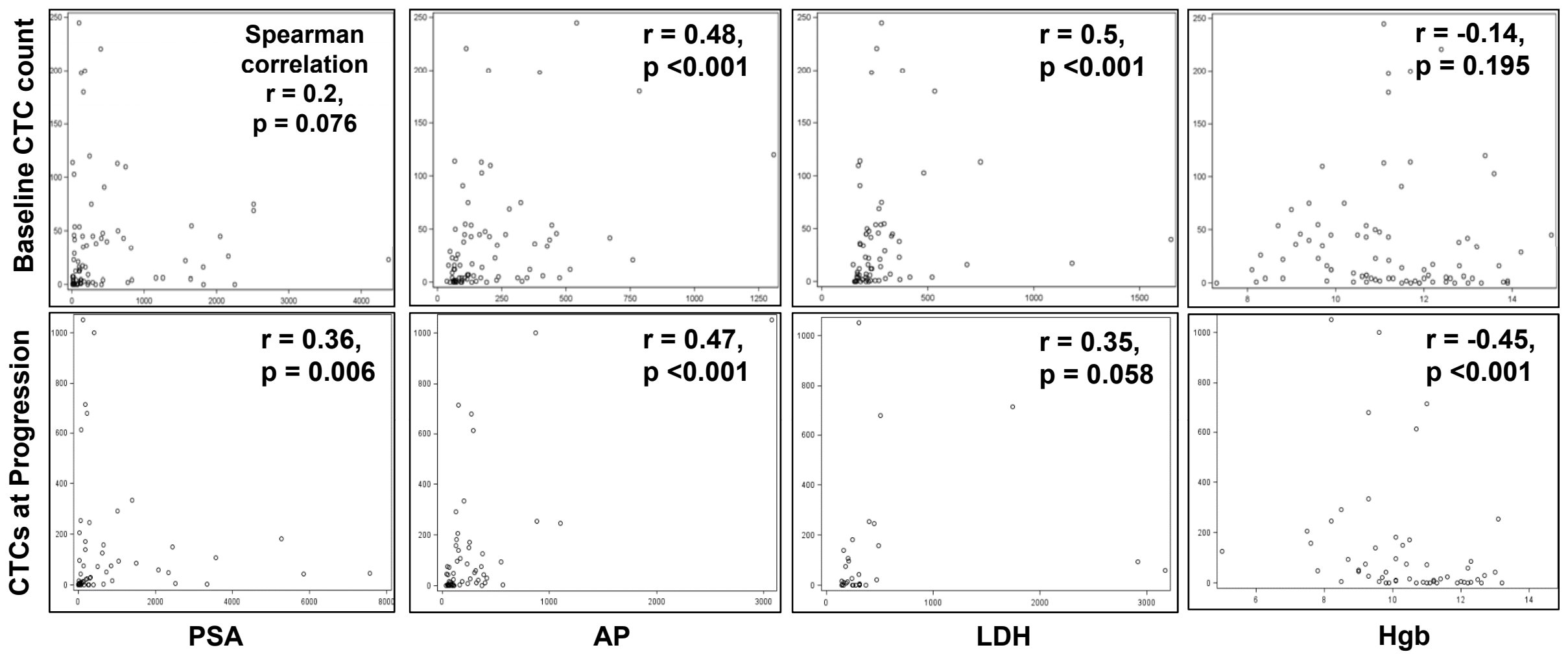


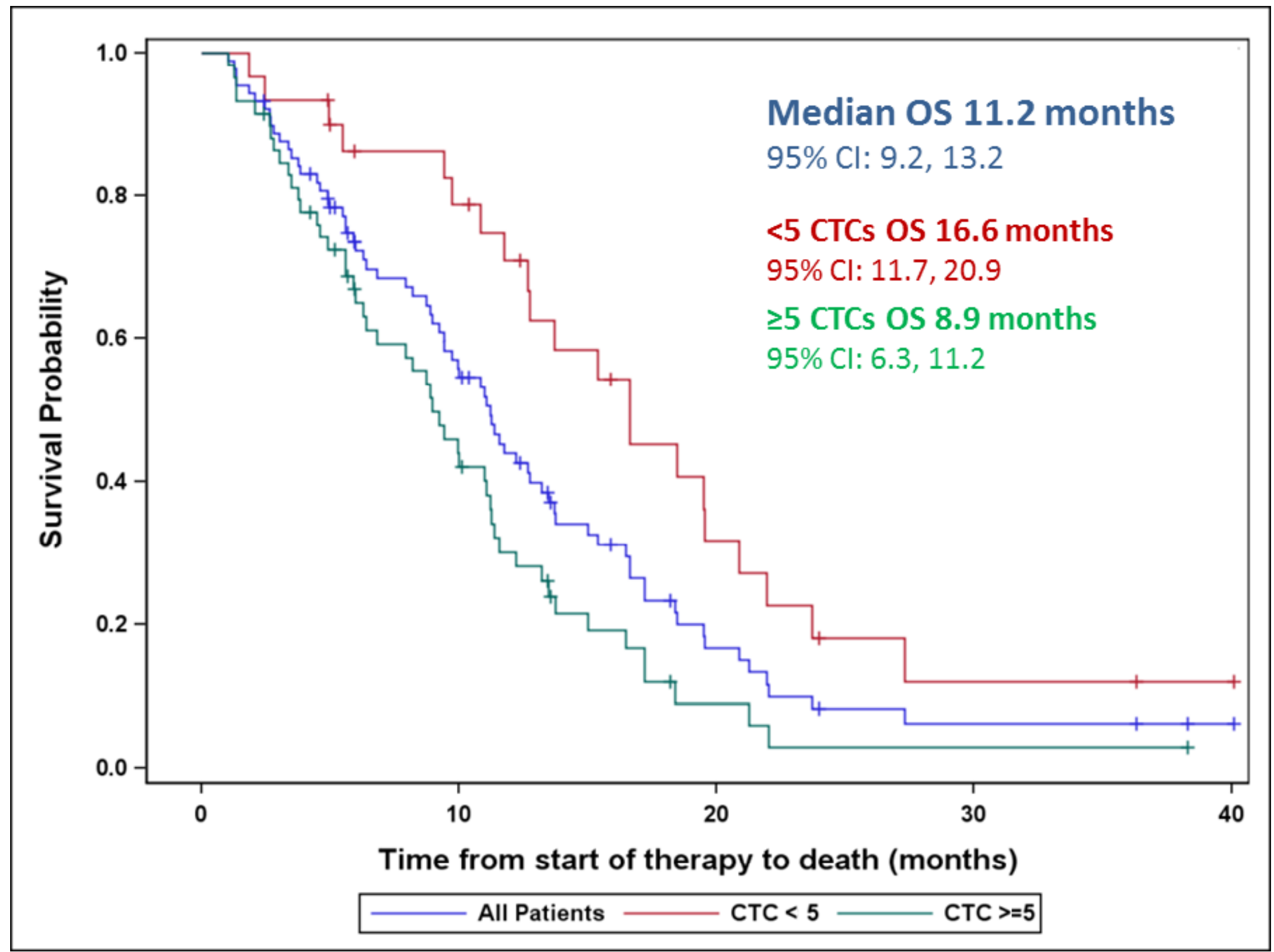




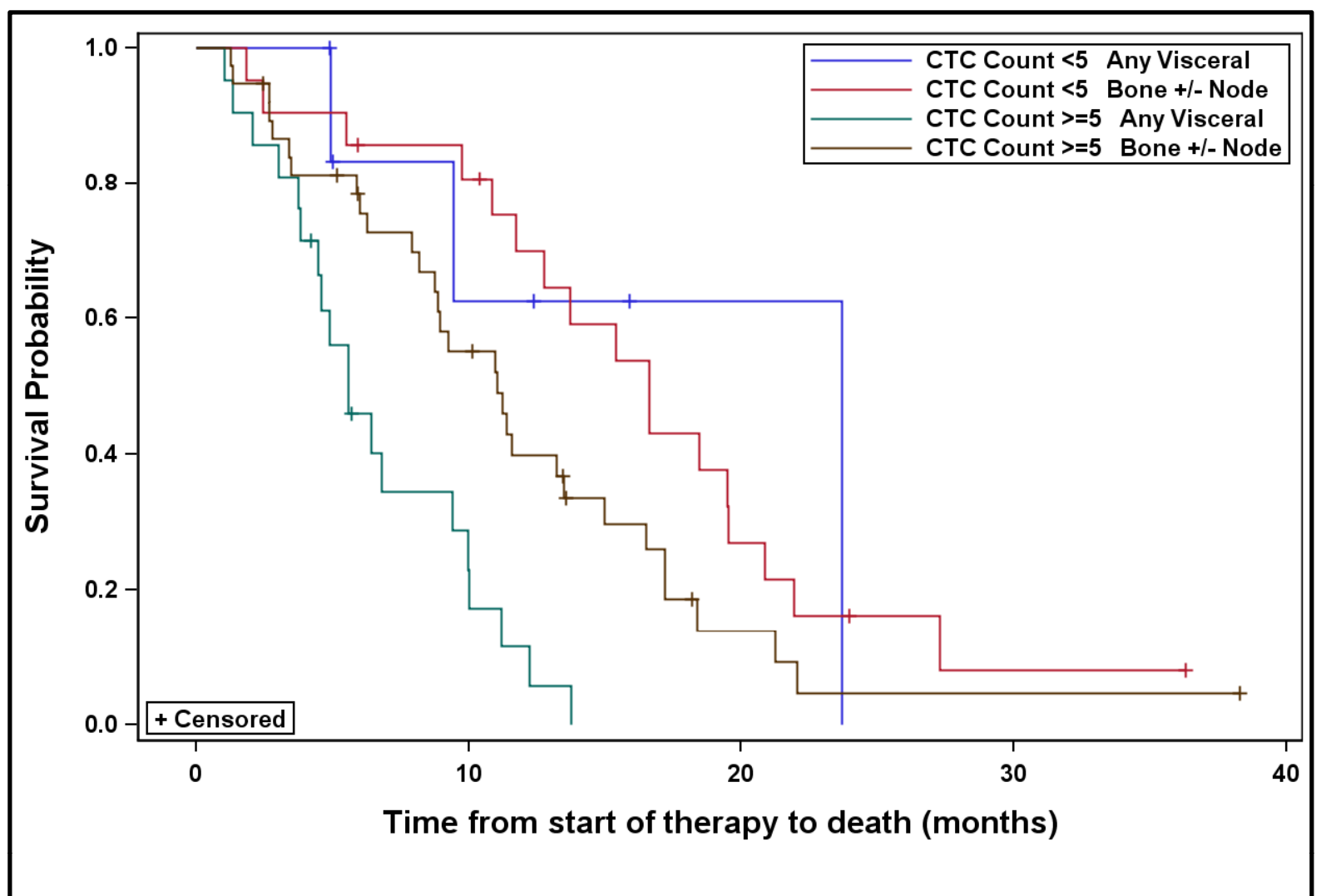

\begin{tabular}{|llll|}
\hline & N & DEATHS & Median OS $(95 \% \mathrm{Cl})$ \\
\hline Visceral metastasis & & & $6.8(4.9,10.0)$ \\
CTC $<5$ & 7 & 3 & $23.7(9.4,23.7)$ \\
CTC $\geq 5$ & 21 & 19 & $5.6(3.8,9.4)$ \\
\hline Bone metastasis & & & $12.7(11.0,16.5)$ \\
CTC $<5$ & 21 & 17 & $16.6(11.7,19.5)$ \\
CTC $\geq 5$ & 38 & 30 & $11.0(8.2,13.5)$ \\
\hline
\end{tabular}




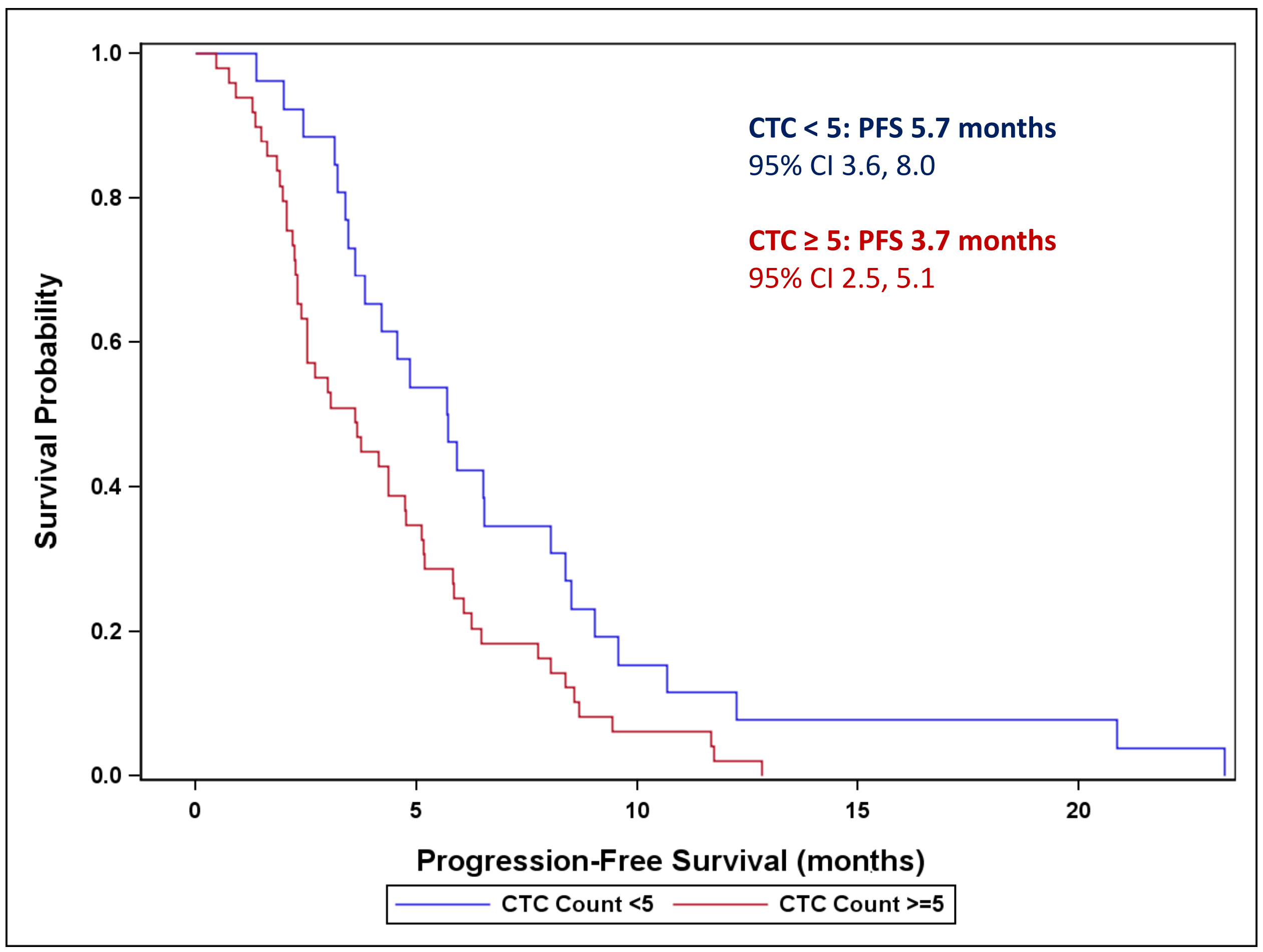




\title{
The role of epithelial plasticity in prostate cancer dissemination and treatment resistance
}

\author{
Rhonda L. Bitting • Daneen Schaeffer • \\ Jason A. Somarelli • Mariano A. Garcia-Blanco • \\ Andrew J. Armstrong
}

(C) Springer Science+Business Media New York 2014

\begin{abstract}
Nearly 30,000 men die annually in the USA of prostate cancer, nearly uniformly from metastatic dissemination. Despite recent advances in hormonal, immunologic, bone-targeted, and cytotoxic chemotherapies, treatment resistance and further dissemination are inevitable in men with metastatic disease. Emerging data suggests that the phenomenon of epithelial plasticity, encompassing both reversible mesenchymal transitions and acquisition of stemness traits, may underlie this lethal biology of dissemination and treatment resistance. Understanding the molecular underpinnings of this cellular plasticity from preclinical models of prostate cancer and from biomarker studies of human metastatic prostate cancer has provided clues to novel therapeutic approaches that may delay or prevent metastatic disease and lethality over time. This review will discuss the preclinical and clinical evidence for epithelial plasticity in this rapidly changing field and relate this to clinical phenotype and resistance in prostate cancer while suggesting novel therapeutic approaches.
\end{abstract}

Keywords Epithelial plasticity · Prostate cancer · Metastasis · Epithelial-mesenchymal transition $\cdot$ Dissemination $\cdot$ Stem cell

\author{
R. L. Bitting $\cdot$ A. J. Armstrong $(\bowtie)$ \\ Division of Medical Oncology, Duke Cancer Institute, Duke \\ University, DUMC Box 102002, Durham, NC 27710, USA \\ e-mail: Andrew.armstrong@duke.edu
}

R. L. Bitting $\cdot$ M. A. Garcia-Blanco $\cdot$ A. J. Armstrong

Department of Medicine, Duke University, Durham, NC, USA

R. L. Bitting • D. Schaeffer · J. A. Somarelli • M. A. Garcia-Blanco •

A. J. Armstrong

Center for RNA Biology, Duke University, Durham, NC, USA

D. Schaeffer · J. A. Somarelli • M. A. Garcia-Blanco •

A. J. Armstrong

Department of Molecular Genetics and Microbiology, Duke

University, Durham, NC, USA

\section{Introduction}

In the USA, nearly 30,000 men die from prostate cancer (PC) each year, largely due to metastatic disease. Although the prognosis for patients with localized disease is good, for patients who develop metastatic disease, the 5-year survival rate is only approximately $30 \%$ [1]. Androgen deprivation therapy (ADT) through either chemical or surgical castration is the first-line therapy for metastatic disease; however, response is temporary, and patients consistently progress to castration-resistant prostate cancer (CRPC), although at variable rates $[2,3]$. The mechanisms underlying castrationresistant progression are likely diverse, but several key pathophysiological themes are emerging, including androgen receptor (AR) amplification, AR splice variants, and mutations in the ligand binding domain that render the AR constitutively active, as well as the induction of autocrine synthesis of androgen precursors within the PC itself [3-5]. In addition, key oncogenic drivers such as activation of the PI3K and Ras signaling pathways, loss of $\mathrm{Rb}$ and $\mathrm{p} 53$ function, and the emergence of epigenetic dysregulation and DNA repair defects underscore the complexity of advanced PC and the multifaceted genomic aberrations that promote treatment resistance.

Emerging from this genetic and epigenetic dysregulation is metastatic and hematogenous dissemination, frequently to bone, but also to other distant sites such as lung or liver. The clinical and pathological phenotype of lethal PC is quite heterogeneous, with autopsy studies demonstrating a high prevalence ( $>90 \%$ ) of bone metastases, and relatively high rates of visceral (liver, lung) metastases (>50\%)[6]. Histologically, metastatic PC is diverse, with some metastases exhibiting a neuroendocrine phenotype, others with poorly differentiated sheets of cells with or without spindle-like cells (sarcomatoid differentiation), and still others with a glandular well-differentiated epithelial appearance. Even 
within patients, phenotypic heterogeneity is commonly observed in histological appearance and protein and RNA biomarker expression, despite an underlying monoclonal metastatic genotype and epigenome [6-9]. These findings suggest substantial cellular plasticity at the level of RNA and protein expression within a given patient that is uncoupled from mutations and chromosomal anomalies. This metastatic dissemination leads to pathological fractures, anemia, bone marrow failure, fatigue, cachexia, progressive pain, and failure to thrive, hallmarks of the lethal clinical phenotype in advanced PC. While available hormonal, immunologic, and chemotherapeutic agents provide palliation and incremental improvements in survival, treatment resistance inevitably emerges over time, and thus, novel approaches are needed in this disease.

One potential approach to understanding metastatic $\mathrm{PC}$ and novel therapeutic strategies is through the study of epithelial plasticity (EP). EP describes the ability of a cell to undergo reversible phenotypic changes during invasion and dissemination. EP encompasses not only the epithelial to mesenchymal transition (EMT) during initial invasion and hematogenous dissemination and its converse of mesenchymal to epithelial transition (MET) during metastatic growth and colonization but also the more general concept of loss of the epithelial phenotype and replacement with a novel phenotype. While EMT is thought to confer upon the carcinoma cell the ability to invade and seed metastatic sites, MET is proposed to enable the disseminated cells to establish macrometastatic colonies. EP is emerging as a common theme in solid tumor pathobiology that encompasses both metastatic dissemination and treatment resistance, with links to underlying embryonal stemness and invasion programs [10]. EMT pathways are causally associated with the acquisition of stem-like properties (the ability to de-differentiate and self-renew) and may link tumor dissemination with phenotypic heterogeneity. Evidence to support EP in cancer biology is robust and has been established in both preclinical models of carcinoma and in patients with carcinomas [11-15]. Furthermore, EP biology has been linked to the risk of metastasis $[10,16]$. In breast cancer models, for example, the induction of an EMT results in the expression of stem cell markers, increased metastatic potential, and resistance to conventional chemotherapy [10, 17-19]. Figure 1 depicts the general concept of EP during PC cellular dissemination. This review describing the role of EP in PC progression will start with a case discussion of secondary neuroendocrine differentiation of prostate cancer.

The concept of EP is illustrated in the following clinical vignette. Patient $\mathrm{X}$ is a 75-year-old African American man, with prostate-specific antigen (PSA) levels that were rising for many years, who presented in March of 2009 with an extremely elevated PSA of 50 . He previously had two prostate biopsies that were negative for malignancy. His third prostate biopsy revealed Gleason $5+5=10$ (high grade) adenocarcinoma with perineural invasion. Imaging revealed enlarged retroperitoneal lymph nodes up to $2 \mathrm{~cm}$ but no visceral or bony metastases. He was treated with combined androgen blockade, and PSA was undetectable within 9 months. Subsequent PSA and imaging progression was treated with sipuleucel-T immunotherapy followed by the novel androgen synthesis inhibitor abiraterone acetate, again with a good PSA response. However, after several months, rapidly enlarging lymph nodes in the setting of a stable PSA prompted a lymph node biopsy. The immunohistochemistry revealed strong staining for CD56 and synaptophysin with minimal PSA, prostatic acid phosphatase (PAP), or cytokeratin staining; together, these findings are suggestive of neuroendocrine differentiation. This neuroendocrine phenotypic transformation was not evident in his original prostate biopsy (Fig. 2). Evolving or secondary neuroendocrine transformation is increasingly recognized in advanced PC $[20,21]$ and may represent one form of EP similar to what has recently been described in lung cancer [22]. It is well documented from autopsy and pathology studies of human PC that many histological phenotypes emerge during hormonal therapy for PC, including squamous differentiation, neuroendocrine differentiation, and a general loss of markers of prostate differentiation [6, 23], as shown in Fig. 2.

Neuroendocrine differentiation (NED) occurs as one path to CRPC [24]. Although NED can arise de novo, it more commonly develops during hormonal therapy for PC [21]. NED does not have a strict clinical or pathological definition, but it is frequently defined histologically as the presence of neuroendocrine cells with chromogranin A or synaptophysin immunoreactivity. Chromogranin A also may be detectable in the plasma, where it correlates with the NED disease burden and is prognostic $[20,25]$. The cells may also stain for synaptophysin or neuron-specific enolase, typically lack AR, and do not secrete PSA [26]. Clinically, NED is suspected when a patient has rapid disease progression, especially with visceral metastases, in the setting of a stable PSA. The presence of NED portends a poor prognosis, with frequent metastasis to the liver, transient response to chemotherapy, and survival often $<1$ year. While NED accounts for a large minority (perhaps $25 \%$ ) of aggressive CRPC [21], other mechanisms of EP leading to phenotypic changes are also likely to be important in human PC dissemination and treatment resistance.

This review focuses on the role of epithelial plasticity in the progression of prostate cancer, from both preclinical and clinical perspectives, and describes how EP may be associated with metastatic dissemination and treatment resistance. Additionally, we provide hypotheses and suggestions for therapeutic interventions to address EP in PC. 


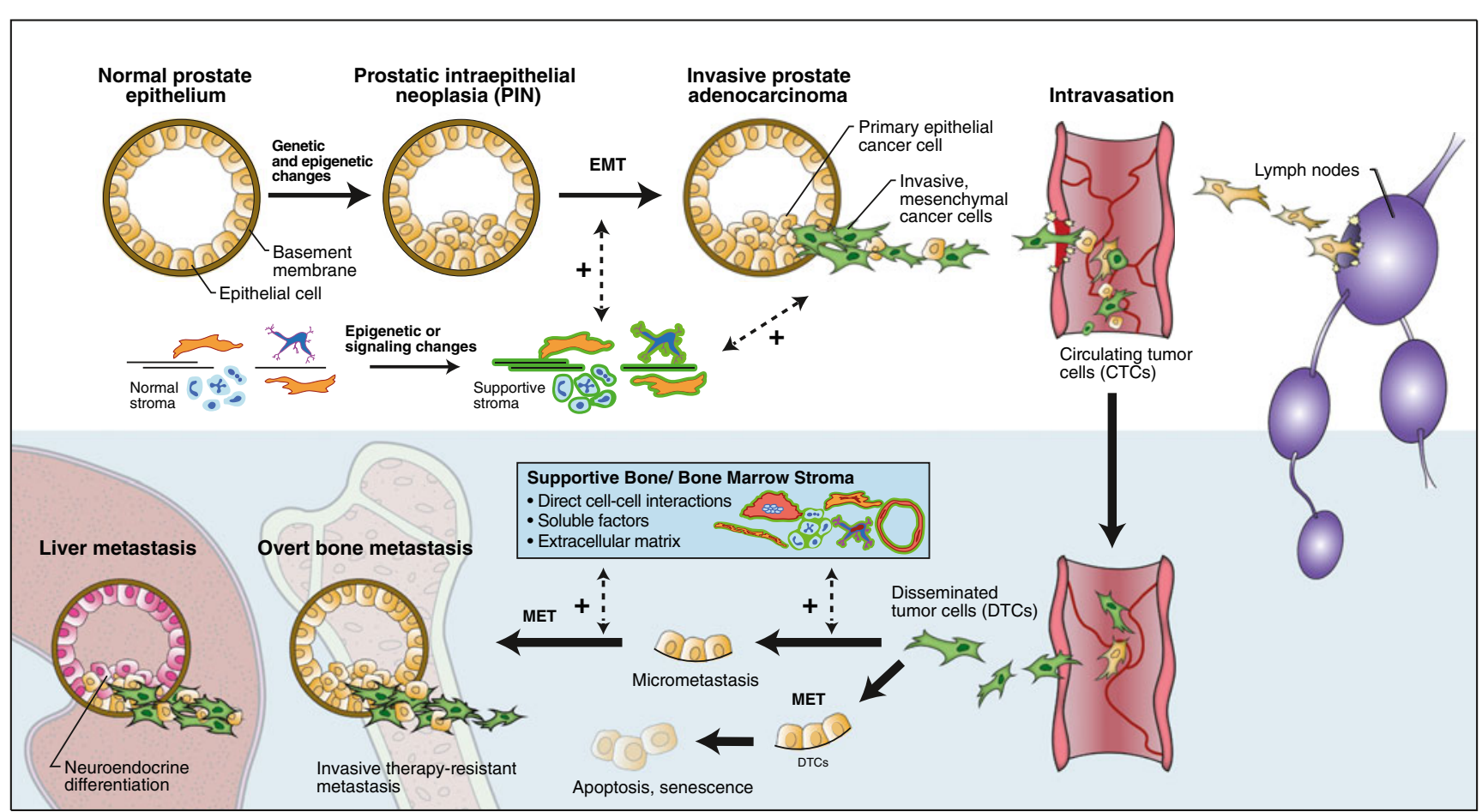

Fig. 1 Epithelial plasticity during prostate cancer dissemination. Due to genetic or epigenetic changes, normal prostate cells begin to grow uncontrollably, a premalignant process known as prostate intraepithelial neoplasia $(P I N)$. In response to signaling from the surrounding stroma, some of these cells undergo an epithelial-mesenchymal transition (EMT) and invade through the basement membrane. These invasive cells enter the bloodstream and may exist as epithelial circulating tumor cells (CTCs), mesenchymal CTCs, or CTCs with a dual phenotype. Upon exiting the vasculature, disseminated tumor cells $(D T C S)$ may sit dormant

\section{Preclinical evidence of EP in PC}

EP in epithelial-origin tumors (carcinomas) involves the reversible loss or reduction of epithelial biomarkers [e.g., Ecadherin, zona-occludens (ZO)-1, cytokeratin isoforms, fibroblast growth factor receptor-2 (FGFR2) isoforms, and miR200 family] and the loss of differentiation antigens [27]. In PC, these differentiation antigens include PSA, PAP, and prostate specific membrane antigen, among others. Epithelial markers may be replaced by mesenchymal markers and transcription factors such as SNAIL, Slug, TWIST1, ZEB1/2, and others, and/or increased expression of stemness pathways, such as Hedgehog or NOTCH signaling. While NED is relatively common in PC progression, it may occur as a result of EP, a fixed evolution through novel mutations, or perhaps both $[21,28]$. Suggesting the importance of plasticity, however, in lung cancer a change to a neuroendocrine-like phenotype can occur in response to treatment and is reversible when treatment is stopped [22]. Also implying the relevance of EP in dissemination and disease progression, at autopsy, many PC patients demonstrate histologic heterogeneity, in which or undergo apoptosis. Other DTCs undergo a mesenchymal-epithelial transition (MET) and grow as detectable macrometastases. In PC, bone metastases are typical and are initially AR dependent, progressing through a range of AR mutations or splice variants, and other oncogenic and tumor suppressor mutations. Visceral metastases are atypical, are variably AR dependent, and generally involve loss of an epithelial phenotype and are enriched for a neuroendocrine or anaplastic phenotype. EP is not clearly linked to the process of lymph node metastasis; instead, nodal metastases likely involve other forms of invasion or migration

multiple phenotypes are evident despite an underlying clonally derived tumor, as shown in Fig. 2.

EMT and MET are highly dynamic and mediated by multiple proteins, microRNAs, and second messengers, including but not limited to those involved in transcription, posttranscriptional gene regulation, signal transduction, cytoskeletal remodeling, migration, invasion, and proliferation. Given the inherent complexity in such a system, it is likely that many incomplete or partial EP-like events take place in different contexts. One such example of an EP-like event is the mesenchymal to amoeboid transition, in which mesenchymal cells are able to alter their cellular shapes to pass through the basement membrane without degrading it [29].

Another type of EP is osteomimicry, in which PC cells can acquire bone-like properties [30]. PC most commonly metastasizes to bones, and the ability of PC cells to mimic the bone environment may enable them to survive and colonize in this new environment. The upregulation of $\beta 2$-microglobulin, an immune regulator protein, can induce EMT, promote osteomimicry, and lead to bone metastasis in mouse models of prostate and other cancers [31]. Furthermore, PC cell lines 

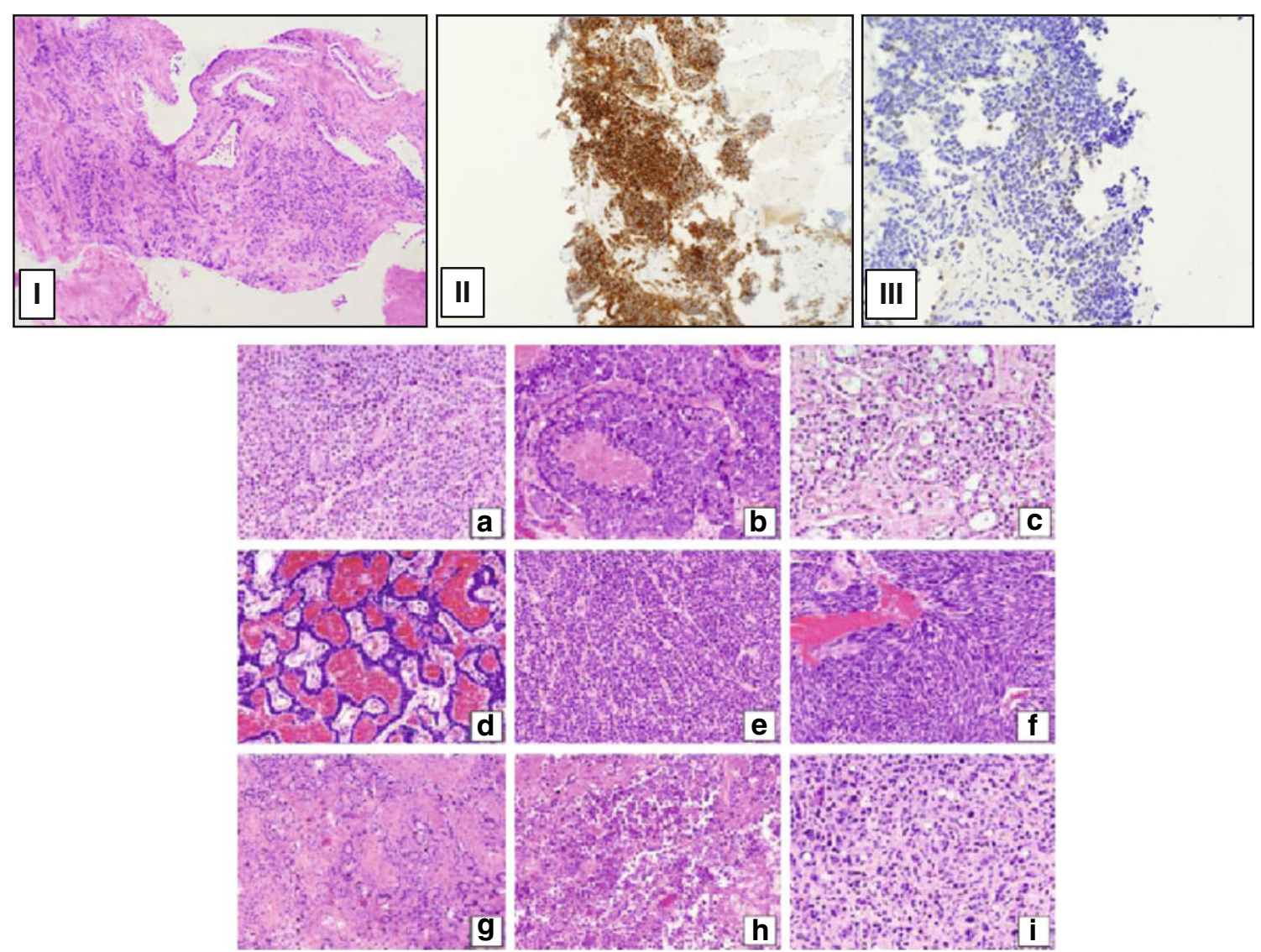

Fig. 2 Examples of prostate cancer phenotypic transformations that emerge with treatment. The top panel is illustrates the phenotypic changes that arise during treatment of patient $\mathrm{X}$, as described in the clinical vignette. His initial prostate biopsy showed high-grade prostate adenocarcinoma (I), but neuroendocrine differentiation emerged as his disease progressed, illustrated by strong synaptophysin (II) with weak PSA staining (III). All images are at $\times 100$ magnification. The bottom panel shows the histological spectrum noted at autopsy of treated prostate cancer. a-c Variations of Gleason grade 4 and 5 adenocarcinoma. d, e Neuroendocrine differentiation. f Small cell carcinoma. $\mathbf{g}$ Well-differentiated Gleason grade 3 disease. $\mathbf{h}$ Undifferentiated growth pattern. i Signet ring differentiation. (Figure reprinted with permission from the American Association for Cancer Research: Rajal Shah et al. [6], p. 9211.) can be forced to differentiate into osteoblast-like cells or adipose cells [32], suggesting that PC cells have the inherent capability to change phenotypes. Additional studies have established that PC cells produce soluble factors that lead to the expression of osteoblast-specific genes [33]. We have identified osteoblast $(\mathrm{OB})$-cadherin frequently in the circulating tumor cells (CTCs) of men with CRPC, illustrating the clinical relevance of this form of phenotypic change [15]. If the process of osteomimicry could be effectively targeted therapeutically, metastasis of PC to bone could potentially be prevented.

A variety of pathways and biomarkers have been confirmed to be associated with EP in cell lines and preclinical xenograft or genetically engineered models of PC; a smaller subset has been validated in human PC progression. Table 1 provides an overview of those pathways and biomarkers linked, preclinically and clinically, to EP in PC. In PC cell lines, EMT can be induced or may occur spontaneously. ARCaP cells, for example, were derived from a patient with metastatic CRPC and gave rise to stable epithelial, $\mathrm{ARCaP}_{\mathrm{E}}$, and mesenchymal,
$\mathrm{ARCaP}_{\mathrm{M}}$, sublines [34]. Other mesenchymal sublines have been generated from a parental epithelial PC line, including derived EPT1 lines, generated by in vitro passaging of the EP156T cell line [35] and the PZ-HPV-7T subline, generated by subrenal capsule xenografting of the PZ-HPV-7 cells [36]. PC-3 and DU145 cells additionally commonly express a range of mesenchymal and epithelial phenotypes [37]. These cell lines are valuable tools for studying EP in PC in the laboratory setting and provide further evidence for EP in clinical settings. The following sections discuss transcriptional activators or repressors of EMT/MET, signaling pathways, microenvironmental cues, microRNA regulators, stemness pathways, and other regulators of phenotypic change and the role that each play in promoting EP and dissemination in PC.

\subsection{Transcriptional activation of EP}

Several transcription factors have been shown to be sufficient for inducing EMT in carcinoma cell lines by repressing the E- 
Table 1 Selected biomarkers and pathways associated with EP in preclinical models and patients with PC

\begin{tabular}{|c|c|c|c|c|}
\hline $\begin{array}{l}\text { Pathway and biomarker associated } \\
\text { with EP in PC }\end{array}$ & $\begin{array}{l}\text { Link to } \\
\text { stemness }\end{array}$ & $\begin{array}{l}\text { Link to } \mathrm{AR} \\
\text { signaling in } \mathrm{PC}\end{array}$ & $\begin{array}{l}\text { Validation in } \\
\text { human PC }\end{array}$ & References \\
\hline \multicolumn{5}{|l|}{ EMT-related transcription factors } \\
\hline SNAIL & $\mathrm{N}$ & $\mathrm{N}$ & $\mathrm{N}$ & {$[38,39,41,42]$} \\
\hline TWIST1 & $\mathrm{Y}$ & $\mathrm{N}$ & $\mathrm{Y}$ & {$[45-51]$} \\
\hline Id-1 & $\mathrm{N}$ & $\mathrm{N}$ & $\mathrm{N}$ & {$[56-61]$} \\
\hline Slug/Snai2 & $\mathrm{N}$ & $\mathrm{Y}$ & $\mathrm{Y}$ & {$[42,43]$} \\
\hline ZEB1/2 & $\mathrm{N}$ & $\mathrm{Y}$ & $\mathrm{N}$ & {$[44,228]$} \\
\hline ETS-family (ERG) & $\mathrm{N}$ & $\mathrm{Y}$ & $\mathrm{Y}$ & {$[225,227-232]$} \\
\hline HIF- $1 \alpha$ & $\mathrm{N}$ & $\mathrm{N}$ & $\mathrm{Y}$ & {$[125-127]$} \\
\hline \multicolumn{5}{|l|}{ Cell surface protein expression } \\
\hline Loss of E-cadherin & $\mathrm{Y}$ & $\mathrm{N}$ & $\mathrm{Y}$ & {$[27]$} \\
\hline $\mathrm{N}$-Cadherin & $\mathrm{Y}$ & $\mathrm{N}$ & $\mathrm{Y}$ & {$[235]$} \\
\hline OB-Cadherin & $\mathrm{N}$ & $\mathrm{N}$ & $\mathrm{Y}$ & {$[15]$} \\
\hline EGFR & $\mathrm{N}$ & $\mathrm{N}$ & $\mathrm{N}$ & [109] \\
\hline FGFR1 & $\mathrm{N}$ & $\mathrm{N}$ & $\mathrm{Y}$ & {$[116,122]$} \\
\hline FGFR2 isoforms & $\mathrm{Y}$ & $\mathrm{N}$ & $\mathrm{N}$ & {$[115-117]$} \\
\hline \multicolumn{5}{|l|}{ Stemness pathways } \\
\hline Hedgehog/NOTCH-1 & $\mathrm{Y}$ & $\mathrm{N}$ & $\mathrm{Y}$ & {$[173,222]$} \\
\hline $\mathrm{WNT} / \beta$-catenin & $\mathrm{Y}$ & $\mathrm{Y}$ & $\mathrm{Y}$ & {$[73-78]$} \\
\hline NANOG & $\mathrm{Y}$ & $\mathrm{N}$ & $\mathrm{N}$ & {$[123]$} \\
\hline BMI & $\mathrm{Y}$ & $\mathrm{N}$ & $\mathrm{N}$ & [199] \\
\hline \multicolumn{5}{|l|}{ TGF- $\beta$ signaling } \\
\hline SMAD4 & $\mathrm{N}$ & $\mathrm{N}$ & $\mathrm{Y}$ & [97] \\
\hline TGF- $\beta$ RIII & $\mathrm{N}$ & $\mathrm{N}$ & $\mathrm{Y}$ & [94] \\
\hline COUP-TFII & $\mathrm{N}$ & $\mathrm{N}$ & $\mathrm{Y}$ & [98] \\
\hline BMPs & $\mathrm{Y}$ & $\mathrm{N}$ & $\mathrm{N}$ & [99] \\
\hline \multicolumn{5}{|l|}{ Intracellular protein signaling } \\
\hline $\mathrm{AR}$ & $\mathrm{N}$ & $\mathrm{Y}$ & $\mathrm{Y}$ & {$[16,64-67]$} \\
\hline PTEN/PI3K pathway & $\mathrm{Y}$ & $\mathrm{Y}$ & $\mathrm{Y}$ & {$[68,69]$} \\
\hline DAB2IP & $\mathrm{Y}$ & $\mathrm{Y}$ & $\mathrm{Y}$ & [79-81] \\
\hline EZH2 & $\mathrm{Y}$ & $\mathrm{Y}$ & $\mathrm{Y}$ & {$[80,166]$} \\
\hline Ras pathway & $\mathrm{Y}$ & $\mathrm{Y}$ & $\mathrm{Y}$ & {$[69,71]$} \\
\hline NF-kB pathway (IL-6/8) & $\mathrm{Y}$ & $\mathrm{Y}$ & $\mathrm{Y}$ & {$[82-87]$} \\
\hline \multicolumn{5}{|l|}{ Micro-RNA species } \\
\hline miR-200 family & $\mathrm{Y}$ & $\mathrm{N}$ & $\mathrm{N}$ & {$[172,174,177]$} \\
\hline \multicolumn{5}{|l|}{ Chaperone proteins } \\
\hline HSP27 & $\mathrm{N}$ & $\mathrm{Y}$ & $\mathrm{Y}$ & {$[14,108]$} \\
\hline
\end{tabular}

cadherin promoter; however, only a few of these transcription factors, including SNAIL, Slug, ZEB1, TWIST1, and Id-1 have been identified as having a role in EMT during PC progression. SNAIL is a zinc finger transcription factor that has been shown to induce EMT in many types of human cancers, including breast [38] and colorectal [39]. Forced expression of SNAIL in epithelial PC lines $\mathrm{ARCaP}_{\mathrm{E}}$ and $\mathrm{LNCaP}$ is sufficient to induce at least a partial EMT, as evidenced by altered biomarker expression and migration. In contrast, SNAIL inhibition in mesenchymal PC-3 cells induces epithelial biomarker expression [40]. Consequently, expression of SNAIL is thought to be both necessary and sufficient to induce EMT, but the relationship of SNAIL to human PC remains to be established. Of note, SNAIL expression also induces a neuroendocrine phenotype in PC cells [41], suggesting that SNAIL expression may play promote differentiation into several cell states. Another zinc-finger transcription factor required for the initiation of EMT in PC cells is Snai2, commonly known as Slug. Knockdown of Slug in PC-3 cells results in increased expression of E-cadherin, suggesting that Slug is required for maintenance of the mesenchymal phenotype [42]. Importantly, Slug acts as a 
coactivator of AR and, in androgen-deprived conditions, provides a growth advantage to PC cells [43]. ZEB1 is another zinc-finger transcription factor that is both necessary and sufficient to induce EMT in PC [44].

TWIST1, a basic helix loop helix (bHLH) transcription factor, has been most widely studied in EMT in breast cancer [45] but has also been shown to induce EMT in gastric [46] and head and neck cancers [47], and is clinically associated with distant metastasis and poor prognosis in these tumor types [48-50]. In PC cell lines, knockdown of TWIST1 has been shown to induce a partial MET with an increase in Ecadherin expression, highlighting the importance of TWIST1 in maintaining a mesenchymal phenotype [51]. Further supporting the role of TWIST1 in EMT is the observation that epigenetic regulation of the TWIST1 promoter is needed for a common p53 mutant to induce EMT. Wild-type p53 is a transcription factor that, when activated by cellular stress, promotes cell cycle arrest and apoptosis [52, 53]. Mutations in p53 are common in cancer cells, are responsible for the functional loss of the tumor suppressor, and may result in downregulation of the epigenetic regulator BMI-1 and resultant upregulation of TWIST1 expression [54]. Dysregulation of p53 is common in metastatic PC, and loss of p53 function may promote EMT through TWIST1 deregulation, or through a separate pathway involving microRNA deregulation [55].

Inhibitor of differentiation/DNA binding (Id-1) is another bHLH transcription factor that has a dominant negative effect on other bHLH transcription factors because it lacks a DNA binding domain. Id-1 is involved in several physiological processes, including inhibition of differentiation and delayed senescence [56], and is upregulated in several carcinomas including prostate [57]. Id-1 interacts with caveolin-1 (Cav-1) [58], which is a membrane protein involved in signaling transduction and is upregulated in metastatic PC [59, 60]. Combined expression of ID-1 and Cav-1 induces cell migration and EMT in LNCaP and PC-3 cells. Specifically, the interaction of Id-1 and Cav-1 induces Akt activation, which is thought to be the mechanism of EMT induction [58]. Cav-1 promotes Akt activation by repressing the activity of a serine/threonine protein phosphatase, PP2A [61], and suppression of PP2A requires Cav-1 binding to PP2A [58]. Together, these results suggest that the interaction between Id-1 and Cav-1 activates Akt and subsequent EMT. Further work in human PC is needed to decipher the relationship between the Id-1 pathway and dissemination/differentiation. Interestingly, NED in human PC has been linked to deregulated PI3K/Akt/mTOR signaling, raising the possibility of a link between EP, the ID-1, and PI3K pathways, and phenotypic transformation $[62,63]$. In summary, a range of transcription factors have been linked in PC cell lines and model systems to EMT and invasion and are typically accompanied by alterations in other cellular pathways important in cellular differentiation, survival, and DNA repair.

\subsection{Signaling in EP}

In preclinical models of PC, transcriptional activation of EP can be induced via a wide range of signaling pathways. Both intracellular activators and soluble growth factors can mediate phenotypic plasticity, and extensive crosstalk between multiple signaling pathways illustrates the importance of redundancy and feedback loops in regulating cellular survival, dissemination, and plasticity. See Table 1 for a select listing of the roles of these pathways in PC progression. In addition, Fig. 3 depicts key signaling nodes in PC that regulate EP.

AR signaling is required for normal development of the prostate [16] and is a common target for therapeutic intervention in PC. The AR pathway is activated by $5 \alpha$ dihydrotestosterone (DHT), a metabolite of testosterone, and binding of DHT to AR initiates translocation of the nucleus, where $\mathrm{AR}$ acts as a transcription factor to transcribe genes involved in cell cycle progression [64]. Importantly, androgens can also modulate EMT in some PC cell lines. For example, treatment of PC-3 and LNCaP cells with DHT leads to downregulation of E-cadherin and upregulation of $\mathrm{N}$ cadherin and SNAIL [16]. Furthermore, knockdown of AR in LNCaP and CWR22 cells sensitizes cells to androgenmediated EMT [16], suggesting that AR may protect PC cells from undergoing EMT in the presence of androgens, whereas AR inhibition may promote EMT. In normal mouse prostate tissue and LuCaP35 xenografts, ADT induces EMT and stemness features [65]. In LNCaP cells, AR represses ZEB1 expression and vice versa [65], indicating that a feedback loop

Fig. 3 Key signaling nodes in prostate cancer that regulate epithelial plasticity. This is a simplified and broad schematic describing the interplay of EP signaling and transcription with AR in aggressive PC. Signaling through multiple and interacting pathways leads to EMT through a variety of mechanisms. Signaling by EGFs, IL6, GAS6, chemokines, and TGF- $\beta$, through their respective receptors, can lead to increased expression of EMT transcription factors (TFs). EMT TFs, including but not limited to TWIST, SNAIL, Slug, and ZEB1/2, can then upregulate mesenchymal biomarker expression (e.g., N-cadherin, vimentin, OB-cadherin) and downregulate E-cadherin expression. TWIST also inhibits FGFR2 expression. These TFs can interact with AR in varying ways. For example, TWIST and Slug can activate AR, while ZEB 1 and AR are reciprocal inhibitors of each other. AR also upregulates NKX3-1, which in turn represses TWIST. When Wnt ligands are present, $\beta$-catenin moves to the nucleus and activates target genes linked to EMT and survival. $\beta$-Catenin can also act as a cofactor with AR. DAB2IP negatively regulates Ras and NF- $\mathrm{KB}$ signaling and, when epigenetically silenced by EZH2, leads to EMT and PC metastasis through activation of the Ras and NF-kB pathways. Loss of DAB2IP also activates AR through phosphorylation by Src kinase and $\beta$-catenin pathways. AR activation can lead to increased TMPRSS2-ERG fusion, which in turn can activate EMT through ZEB1/2 and increase $\beta$-catenin signaling. FGFs signal through the PI3K/Akt pathway to promote tumor proliferation, and the PI3K/Akt pathway also negatively regulates AR. DHT is the AR ligand, and when available to tumor cells, also promotes growth. Note that not all pathways discussed in the manuscript are shown in this figure 


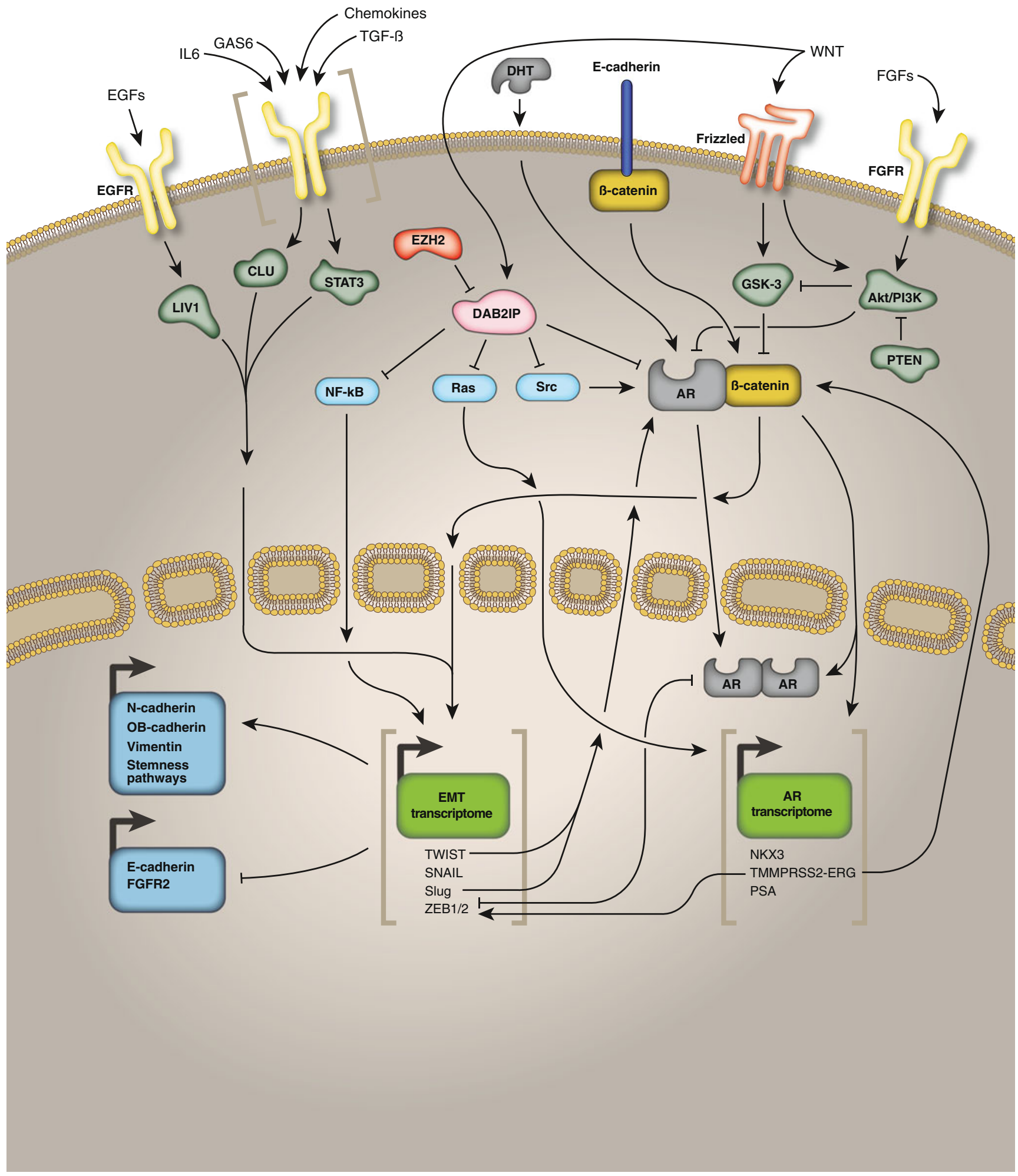

between these two proteins may exist. AR also upregulates NKX3-1, which represses TWIST1 via binding to the TWIST1 promoter [66]. Contrary to the above findings, which suggest that AR inhibits EMT, ectopic expression of AR in BPH-1 cells induces EMT, whereas knockdown of AR downregulates EMT markers [67], suggesting that AR may play a different role in culture conditions than within the tumor microenvironment. The connections between AR signaling and EP are likely complex and context dependent, and many signaling pathways including $\beta$-catenin, Src kinase, 
Akt/mTOR, and G-protein receptors can signal directly to AR independent of ligand, further adding to the complexity. Tables 1 and 2 provide an overview of these associations.

Loss of PTEN, a phosphatidylinositol-3,4,5-trisphosphate 3-phosphatase and member of the Akt signaling pathway, is observed in approximately $40 \%$ of human PC [68]. Genetic knockout of PTEN in mouse models mirrors the clinical disease course but without progression to metastatic disease [69]. To identify the additional components required for metastatic disease progression, an analysis of human PC microarrays revealed that the Ras pathway is significantly upregulated in both primary and metastatic PC tissue [69]. Interestingly, a prostate-specific Ras/PTEN-null mouse model results in PC, followed by EMT and metastasis in $100 \%$ of mice. Models with PTEN null or Ras mutant tumors alone do not develop macrometastases, suggesting the importance of cooperative signaling in the promotion of dissemination [69]. PTEN loss is linked to the acquisition of stemness properties and loss of a differentiated phenotype in PC model systems $[69,70]$. Given that aberrations in the PTEN/PI3K, AR, and Ras signaling pathways are present in nearly $100 \%$ of metastatic PC [71], it is likely that drivers of EP are associated with these three key oncogenic pathways in CRPC.

The wingless/int1 (Wnt) gene was originally identified as a retroviral oncogene and a modulator of embryonic development in Drosophila melanogaster [72]. Decades later, it was shown that anomalous activation of the $\mathrm{Wnt} / \beta$-catenin pathway is a driver of multiple human cancers, including prostate [73]. The Wnt pathway is activated by the binding of Wnt ligands to their receptors. When Wnt ligands are present, $\beta$-catenin moves to the nucleus and activates target genes linked to EMT, invasion, proliferation, and survival [74]. In PC, $\beta$-catenin may act as cofactor with AR [75], and increased $\beta$-catenin expression and change in localization have been observed in advanced disease [76, 77]. Another member of the Wnt family, Wnt5a, mediates EMT via activation of the membrane type I matrix metalloproteinase (MT1-MMP), which is a membrane-bound MMP involved in degrading the extracellular matrix, and is upregulated in breast and prostate cancers [78].

Also involved in the Wnt pathway, DAB2IP, a Ras GTPase-activating protein, has been shown to possess tumor suppressive properties via maintenance of an epithelial phenotype [79]. Knockdown of DAB2IP leads to EMT in PC-3 cells, while overexpression of DAB2IP decreases mesenchymal biomarker expression and migratory potential of PC cells via antagonism of the $\mathrm{Wnt} / \beta$-catenin pathway. Moreover, knockdown of DAB2IP in PC-3 cells leads to increased metastatic burden in a xenograft mouse model [79]. Importantly, DAP2IP levels positively correlate with E-cadherin and negatively correlate with vimentin in primary tumor tissue from PC patients [79], which supports the role of the Wnt pathway in mediating PC progression via regulation of EP. Epigenetic loss of DAB2IP has been linked to EMT and PC metastasis through overexpression of the epigenetic regulator EZH2 and subsequent downstream activation of nuclear facto kappa $\mathrm{B}(\mathrm{NF}-\mathrm{kB})$ and Ras pathways [80]. Furthermore, the loss of DAB2IP is linked to enhanced AR activation and AR variant activity through phosphorylation by Src kinase and $\beta$-catenin pathways, providing a novel

Table 2 Selected clinical states of PC and evidence of associations with EP as a treatment resistance mechanism

\begin{tabular}{|c|c|c|c|}
\hline Clinical Disease State of PC & Description of EP Association with Outcome & $\begin{array}{l}\text { Direct evidence from } \\
\text { men with PC }\end{array}$ & References \\
\hline \multicolumn{4}{|l|}{ Localized disease } \\
\hline \multirow[t]{2}{*}{ Surgery (radical prostatectomy) } & $\begin{array}{l}\mathrm{E} / \mathrm{N} \text { cadherin switch associated with PSA recurrence, metastasis } \\
\text { after surgery }\end{array}$ & $\mathrm{Y}$ & [223] \\
\hline & $\begin{array}{l}\text { Loss of CK or PSA expression, increased TWIST or vimentin in } \\
\text { localized disease correlates with outcomes }\end{array}$ & $\mathrm{Y}$ & {$[221,224]$} \\
\hline Radiation therapy & Induction of WNT16B in stroma mediates radioresistance in PC & $\mathrm{N}$ & {$[150]$} \\
\hline Active Surveillance & $\begin{array}{l}\text { ERG overexpression in biopsy specimens associated with } \\
\text { progression during surveillance }\end{array}$ & $\mathrm{Y}$ & {$[229]$} \\
\hline \multicolumn{4}{|l|}{ PSA recurrent disease } \\
\hline Androgen deprivation therapy & ADT induction of EMT transcription factors & $\mathrm{Y}$ & {$[65]$} \\
\hline \multicolumn{4}{|l|}{ Metastatic PC } \\
\hline Immunotherapy & $\begin{array}{l}\text { Immunotherapy against epithelial targets leads to mesenchymal } \\
\text { tumor escape }\end{array}$ & $\mathrm{N}$ & [240] \\
\hline Docetaxel chemotherapy & $\begin{array}{l}\text { Loss of CK, overexpression of stemness pathways (NOTCH/ } \\
\text { Hedgehog) in docetaxel-treated metastases, PC cell lines }\end{array}$ & $\mathrm{Y}$ & {$[200]$} \\
\hline Cytotoxic DNA-damaging agents & $\begin{array}{l}\text { Induction of DNA-stress response in stroma leads to WNT16b } \\
\text { induction and EMT, treatment resistance to mitoxantrone }\end{array}$ & $\mathrm{Y}$ & {$[150]$} \\
\hline Circulating tumor cell expression & $\begin{array}{l}\text { Common expression of vimentin, N-cadherin, CD133, } \\
\text { OB-cadherin in CTCs from men with metastatic CRPC }\end{array}$ & $\mathrm{Y}$ & {$[15]$} \\
\hline
\end{tabular}


link between EMT, dissemination, and AR signaling mediated through the epigenetic and thus reversible loss of DAB2IP [81].

NF- $k B$ transcription factors regulate a variety of immune and inflammatory responses and developmental processes (reviewed by [82]). Levels of NF-KB correlate with prognosis in PC patients, and increased NF- $\mathrm{KB}$ signaling correlates with disease progression in a subset of PC patients [83]. NF- $\mathrm{kB}$ regulates EMT by directly or indirectly upregulating multiple EMT transcription factors, the mesenchymal intermediate filament protein vimentin, and matrix metalloproteases MMP2 and MMP9 [84]. In addition, IkappaB kinase alpha activation by receptor activator of NF-KB ligand (RANKL) inhibits expression of the Maspin protein and metastatic dissemination. Maspin is a serpin family member, expression levels are inversely correlated with metastatic potential in human PC, and its signaling or epigenetic regulation may be causally related to dissemination [85]. In PC cell lines, induction of EMT leads to upregulation of RANKL [86]. Interestingly, RANKL activation results in osteoclastogenesis in vitro [86], suggesting that upregulation of RANKL via EMT induction may promote skeletal metastasis. NF- $\mathrm{kB}$ also mediates EMT via downregulation of fibulin and activation of CXCL1/ GRO $\alpha$ [87], a chemokine that promotes angiogenesis and enhances cancer cell proliferation [88]. These examples highlight the complexity of signaling networks that may cooperate to drive EMT and the metastatic cascade in advanced disease.

One of the best-studied initiators of EMT is the transforming growth factor beta (TGF- $\beta$ ) family of cytokines and their receptors, TGF- $\beta$ RI, II, and III. TGF- $\beta$ can induce EMT, as evidenced by increased expression of mesenchymal biomarkers in multiple PC cell lines [89]. Importantly, TGF- $\beta$ can induce EMT in an androgen-independent cell line, PC-3, and in an androgen-dependent line, LNCaP, suggesting that the ability of TGF- $\beta$ to induce EMT is independent of AR expression [90]. TGF- $\beta$ treatment also induces clusterin (Clu) expression during EMT, with Clu functioning as a molecular chaperone to protect against cellular stresses [91]. Clu is transcriptionally activated by TWIST1, and this activation is required for TGF- $\beta$-induced EMT [89]. Clu has emerged as an important therapeutic target in men with CRPC, and given its role in mediating chemotherapy resistance, its link to EP may be equally important $[92,93]$. In addition, loss of TGF- $\beta$ RIII is common in human PC, through deletions or epigenetic dysregulation, and this is accompanied by enhanced invasion and relapse after surgery [94]. The paradox of TGF- $\beta$ signaling in human PC, in which there is increased TGF- $\beta$ expression and tumor suppression early in the disease, followed by tumor promotion during disease progression, may be explained through altered intracellular signaling. Specifically, TGF- $\beta$ signaling may initially promote invasiveness and escape from the primary tumor microenvironment; however, loss of TGF- $\beta$ in distant metastasis may promote an epithelial phenotype and ultimately colonization $[95,96]$. For example, loss of SMAD4 is consistently identified in metastatic as compared to localized PC, indicating that loss of this tumor suppressor may facilitate dissemination [97]. Importantly, SMAD4 was identified as a component of a four-gene signature, along with PTEN, cyclin D1, and SPP1, that is prognostic of biochemical recurrence and metastatic disease in human PC [97]. It has recently been shown that COUP transcription factor II (COUP-TFII) regulates SMAD4dependent transcription in PTEN-null tumors, making a TGF- $\beta$ dependent checkpoint ineffective and leading to EMT and metastasis [98]. Taken together, loss of SMAD4 signaling and altered TGF- $\beta$ signaling is associated with the acquisition of an invasive phenotype and metastatic dissemination in PC. Finally, a TGF- $\beta$ superfamily member, bone morphogenetic protein-7, protects against bone metastases in PC through the induction of epithelial differentiation [99], possibly by counteracting SMAD family members. However, the role of BMPs and TGF- $\beta$ signaling in general in mediating $\mathrm{EP}$ and $\mathrm{PC}$ dissemination remains an area of active investigation.

The role of the interleukin-6 (IL-6)/STAT3 pathway, which activates inflammatory responses during infection and oncogenesis $[100,101]$, in EMT has been demonstrated in head and neck [102], nonsmall cell lung [103], and breast cancers [104]. This pathway may also be important in PC, as IL-6 can induce EMT in some PC cell lines. Importantly, induction of EMT by IL-6 requires Hsp27 expression. Specifically, knockdown of Hsp27, an ATP-independent molecular chaperone that is induced in response to stress [105-107], reverses the pro-EMT effect of IL-6. The role of Hsp27 in IL-6-induced EMT is likely through the transcriptional activation of TWIST1. Hsp27 expression is required for TWIST1 expression upon treatment with IL-6, and transcriptional activation is mediated by direct binding of STAT3 to the TWIST1 promoter [108]. Taken together, this suggests that Hsp27 is needed for IL-6-induced EMT but also can act independently to induce EMT. IL-6 has also been linked to activated stromal and immune cell cross-talk and induction of EP/stemness in PC [14], suggesting a complex relationship between the tumor microenvironment and EP.

Other pathways implicated in PC progression preclinically through an EP mechanism include the following: (1) the epidermal growth factor receptor (EGFR) pathway via expression of LIV-1, a zinc transporter [109]; (2) macrophage inhibitory cytokine- 1 , a member of the TGF- $\beta$ superfamily that plays a key role in regulating growth and differentiation in response to stress [110-112]; (3) ß2-microglobulin mediation of the hemochromatosis protein, a member of the nonclassical major histocompatibility complex signaling pathway [31]; (4) the kallikrein family of serine proteases, which induce EMT and invasiveness [113]; and (5) ubiquitin C-terminal hydrolase-L1, 
UCH-L1, a deubiquitinating enzyme, the expression of which is both necessary and sufficient to induce EMT [114].

\subsection{Alternative splicing in EP}

There is evidence that fibroblast growth factor receptor (FGFR) signaling plays a role in PC onset and progression. The FGFRs are a family of four receptor tyrosine kinases (FGFR1-4) that bind to a family of fibroblast growth factors (FGFs) (reviewed in [115]). Binding of FGFs induces dimerization of the receptors and signaling via MAPK and PI3K/Akt pathways. FGFR1-3 transcripts are alternatively spliced within their ligand binding domains to give IIIb and IIIc isoforms. The IIIb and IIIc isoforms are typically expressed exclusively in epithelial and mesenchymal cells, respectively [115]. A switch from FGFR2-IIIb to FGFR2IIIc in nontumorigenic rat prostate epithelial cells leads to malignancy [116]. A subset of human PC specimens expresses the IIIc isoform, although metastatic samples predominantly express the epithelial IIIb isoform [117]. Work from our group and others has identified several splicing factors that regulate FGFR2 isoform switching, including PTBP1 [118], RBFOX2 [119], and ESRP1 [120]. Interestingly, RBFOX2 and ESRP1 have also been implicated in mediating numerous splicing events that help to maintain mesenchymal or epithelial phenotypes, respectively, in breast cancer cell lines [121]. It is possible that these splicing factors may play a role in EP during PC by inducing FGFR2 isoform switching and by regulating the splicing of a number of other EP-related transcripts.

Both FGFs and FGFRs are known to be upregulated in PC, including FGFs 1, 2, 6, 7, 8, and 9 and FGFR1 [115], and inducible expression of FGFR1 leads to adenocarcinoma and EMT in a mouse model of PC [122]. FGFR1-induced adenocarcinomas show loss of the epithelial-specific IIIb isoform, increases in Sox9, MMP15, and genes related to TGF- $\beta$ signaling, and metastases to the liver and lymph [122]. The lack of validated FGFR isoform-specific antibodies has impaired the translation of these findings in human PC, and this work is ongoing.

\subsection{Microenvironmental cues as mediators of EP}

The effect of hypoxia within the tumor microenvironment on EP has been widely studied in human cancer. Hypoxia is capable of inducing EMT in PC-3 and LNCaP cells, as evidenced by a switch to a more mesenchymal morphology and increase in mesenchymal biomarker expression [90]. Additionally, PC-3 cells grown under hypoxic conditions have an increased migratory and invasive phenotype. Hypoxia also induces transcripts associated with stemness, including Nanog and EZH2 in PC-3 and LNCaP cells [123].
The molecular mechanism of hypoxia-induced EMT is through the stabilization of HIF- $1 \alpha$, a transcription factor expressed at low oxygen concentrations [124]. Importantly, PC-3 cells grown under hypoxic conditions have increased HIF- $1 \alpha$ protein expression [123] and forced expression of HIF- $1 \alpha$ in LNCaP cells results in a partial EMT, as evidenced by a decrease in E-cadherin expression and an increase in vimentin $[125,126]$. In addition, in other models systems, HIF- $1 \alpha$ expression increases TWIST1 transcription to promote EMT and metastasis $[127,128]$. To date, however, regulation of TWIST1 by HIF- $1 \alpha$ in PC has not yet been studied.

Hypoxia also plays an indirect role in the initiation of the EMT cascade by stabilizing the Axl/GAS6 axis. Axl is a receptor tyrosine kinase that induces cell survival/ proliferation upon binding its ligand, GAS6. The Axl/GAS6 pathway is important for metastasis of several carcinomas [129-133], and is adversely prognostic [134-138]. Axl is necessary for EMT, as evidenced by reduction in mesenchymal biomarkers and increased migration and invasion upon knockdown of Axl in PC cells [139]. GAS6 downregulates expression of its receptor, Axl, and hypoxia is sufficient to prevent GAS6-mediated downregulation of Axl. Therefore, hypoxia acts to stabilize Axl/GAS6 signaling, which ultimately results in induction of EMT [139].

Another mechanism by which the tumor microenvironment can contribute to EP is by fibroblasts in the host stroma, which secrete soluble factors, such as growth factors and extracellular matrix $[140,141]$. Activated fibroblasts (AFs) are necessary for the growth and differentiation of PC cells $[142,143]$, and AF can induce EMT. Specifically, prostatespecific fibroblasts isolated from men with benign hyperplasia and can be activated by either TGF- $\beta$ treatment or by exposure to conditioned media from PC-3 cells to induce EMT. EMT induction in PC-3 cells also promotes stemness, as evidenced by an increase in prostasphere formation, an increase in CD133 positive cells, and an increase in the percentage of $\mathrm{CD} 44^{\text {high }} / \mathrm{CD} 24^{\text {low }}$ cells [14]. Furthermore, induction of EMT in PC-3 cells activates the COX-2 pathway and HIF1A, both of which are involved in the inflammatory response. Upon knockdown of COX-2 and HIF1A in PC-3 cells, EMT cannot be induced, suggesting that the proinflammatory axis is required for initiation of EMT. In addition to initiating an inflammatory response, induction of EMT in PC3 cells also results in reactive oxygen species (ROS) production. With inhibition of ROS production by treatment with antioxidants, prostate AF can no longer induce EMT, stemness, or the inflammatory response pathway [144]. Together, these data suggest that prostate AF produce ROS and activate the proinflammatory response to induce EMT and stemness [14, 144].

The generation of ROS has been associated with EMT in several model systems, including human ovarian carcinoma 
cells [145], renal tubular epithelium [146], and mammary epithelial cells [147]. In the context of PC, there are conflicting reports about the role of ROS in mediating EP. For example, ROS increase during SNAIL-induced EMT, and a ROS scavenger, $\mathrm{N}$-acetyl cysteine, causes a partial reversion of EMT [148]. On the contrary, psoralidin, a natural pro-oxidant chemical, induces ROS production, but leads to downregulation of $\beta$-catenin and Slug, upregulation of Ecadherin, and inhibition of migration and invasion in PC cell lines [149]. While it remains unclear whether ROS stimulates or prevents EMT, it is possible that different ROS levels can have variable effects on the phenotypic status of a cell. For example, moderate ROS can induce cell proliferation, but higher levels lead to apoptosis ([149] and references therein). In addition to hypoxia and ROS, stromal cells can induce EMT through a range of soluble mediators such as chemokines and the soluble protein WNT16B. DNA damage from radiation or chemotherapy can to induce WNT16B and promote EMT in the neighboring prostate epithelial cells, leading to invasion and treatment resistance [150]. Furthermore, activated fibroblasts and other stromal cells such as fat cells or bone marrow derived cells may be recruited into the prostate from distant sites to promote EP [151]. Thus, a number of microenvironmental and host insults can promote EP, dissemination, and treatment resistance in PC. In addition to microenvironmental drivers of EMT, there is also evidence that MET in metastatic colonization may be mediated by the microenvironment. For example, DU-145 cells re-express Ecadherin upon coculture with human hepatocytes, and reexpression of E-cadherin also leads to chemoresistance, suggesting that MET may serve a protective role against chemotherapeutics at metastatic sites [152]. Similarly, coculture of DU-145 and PC-3 cells with primary rat hepatocytes leads to re-expression of E-cadherin and cytokeratin and reduced levels of vimentin [153], and coculture of $\mathrm{ARCaP}_{\mathrm{M}}$ cells with bone marrow stromal cells results in re-expression of $\mathrm{E}$ cadherin in the $\mathrm{ARCaP}_{\mathrm{M}}$ cells [154], lending further support for the idea that microenvironmental cues at the sites of metastatic dissemination may lead to MET. Using a reporter of MET based on alternative splicing of a mesenchymal IIIc exon of FGFR2, clusters of MET can be identified within AT3 Dunning rat mesenchymal prostate tumors [155]. These regions of MET also express E-cadherin and ZO-1 and localize to areas rich in collagen, suggesting that the interaction of tumor cells with collagen or some other microenvironmental driver may contribute to MET.

\subsection{Epigenetics in EP}

Histone deacetylase inhibitors (HDACI) have been studied as potential cancer therapeutic agents based on the increased expression and activity of HDACs in carcinomas (as reviewed in [156]). When evaluating the efficacy of HDACI in PC cell lines, the cells unexpectedly undergo EMT upon treatment with both suberoylanilide (SAHA) and trichostatin A (TSA), as evidenced by a more mesenchymal morphology, upregulation of ZEB1 and vimentin, and increased stemness and migration. The mechanism by which HDACI induce EMT is thought to be via hyperacetylation of EMT promoters, which create a more relaxed chromatin state to promote transcription. Specifically, PC-3 cells treated with TSA and SAHA have an increased amount of acetylated histone 3 associated with the vimentin, ZEB2, and slug promoters, which results in increased EMT signatures [157]. These findings may explain the limited single agent activity of HDACIs in the clinic as therapy for CRPC and suggests that combination approaches are needed [158].

Despite the limited utility of HDACI in clinical treatment of PC, there is evidence for the importance of epigenetic changes in PC. For example, multiple myeloma SET domain (MMSET), a histone methyltransferase that is associated with the dimethylation of histone $\mathrm{H} 3$ lysine 36 , a mark of active transcription [159], can be upregulated in PC [160], with high expression associated with PC recurrence [161]. Overexpression of MMSET in PC cells leads to increased expression of mesenchymal biomarkers and a more migratory and invasive phenotype. Conversely, knockdown of MMET in PC cells leads to decreased migration and invasion. MMSET promotes EMT by binding the TWIST1 promoter and increasing TWIST1 transcription, suggesting that MMSET epigenetically regulates TWIST1 to induce EMT [162].

SIRT1 is another histone deacetylase, which is implicated in the stress response [163] and apoptosis [164] and induces EMT in PC cells. Moreover, knockdown of SIRT1 in PC cells induces MET. ZEB1 is required for SIRT1 to induce EMT, as ZEB1 recruits SIRT1 to the E-cadherin promoter for deacetylation of histone $\mathrm{H} 3$, which suppresses E-cadherin transcription. This suggests that ZEB1 interacts with SIRT1 to downregulate the E-cadherin promoter to induce EMT [165]. Likewise, enhancer of zeste homolog 2 (EZH2), which is involved in gene silencing by histone methylation, is overexpressed in advanced PC and can mediate the silencing of E-cadherin [166]. Interestingly, a survey of primary PC samples and metastatic bone biopsies showed that $70 \%$ of primary PC samples have a methylated E-cadherin promoter with heterogeneous E-cadherin expression, while $87 \%$ of metastatic bone biopsies contain an unmethylated E-cadherin promoter with homogenous E-cadherin expression [167]. Together, these results demonstrate that EMT can be epigenetically regulated and provide a mechanism linking EMT with PC progression.

\subsection{MicroRNAs in EP}

MicroRNAs (miRs) are important regulators of gene expression that play diverse roles in development, metabolism, and 
the pathogenesis of cancer (as reviewed in [168, 169]). Several miRs have been shown to regulate EP, including miR-21, miR-31, miR-29a, miR-135, and the miR-200 family (reviewed in [170]). In PC, ectopic expression of miR-1 or miR-200 precursors reduces Slug-dependent EMT, restores E-cadherin expression, and significantly reduces the invasive potential of PC-3 cells [171]. Similarly, PC-3 cells overexpressing platelet derived growth factor $\mathrm{D}$ undergo EMT, which leads to reduced levels of miR-200 family members [172]. Re-expression of miR-200b induces MET [172] and represses NOTCH1, a driver of stemness [173]. Taken together, these data suggest that miR-200b acts as a tumor suppressor at least partially through regulation of NOTCH1 expression.

Loss of the ZEB1 and ZEB2 repressors, miR-200c and miR-205, has been shown in docetaxel resistant PC-3 and DU145 lines and re-expression of either miR led to Ecadherin upregulation [174]. This suggests that loss of these miRs during docetaxel-mediated EMT may contribute to chemotherapeutic resistance. Additional studies have shown that expression of miR-182, miR-203, and miR-29b in mesenchymal prostate cells can induce MET [175]. While many miRNAs have been associated with MET, miR-21 has been shown to induce EMT in RWPE-1 cells [176] and is the only mesenchymal-specific miRNA currently identified in human PC.

Although there are a number of in vitro studies on miRs in PC, few studies have investigated levels of EP-related miRs in PC specimens. While both miR-200c and miR-29 contribute to an epithelial phenotype in vitro, the correlation between these miRNAs and clinical outcome is less clear. For example, the epithelial specific miRNAs, miR-200c and miR-29b, are upregulated in men with CRPC compared to those with localized disease [177] and in patient-matched normal tissue compared to PC [178]. This is inconsistent given that an epithelialspecific miRNA is associated with both metastasis and healthy prostate tissue. Similarly, the mesenchymal-specific miRNA miR-21 is higher in CRPC tissues compared to localized PC [177]. One possible explanation is that an epithelial phenotype can be simultaneously associated with normal prostate tissue and also be needed for metastatic colonization via MET. It is conceivable that the mesenchymal miR-21 is associated with an early metastatic event, while miR-200c is associated with a late metastatic event that requires MET for colonization. Further complicating the relationship between miRs, EP, and clinical outcome, the loss of epithelial-specific miR-205 reduces time to biochemical recurrence in human PC [179].

\subsection{Dietary and small molecule control of EP in PC}

A number of dietary substances and small molecules can induce epithelial differentiation (MET) and possibly invasion in PC cell lines. One of the most frequently cited supplements is silibinin, the active compound in milk thistle extract, which has shown some promise as a regulator of EP. Silibinin treatment inhibits growth of PC cell lines [180], synergizes with various chemotherapeutic compounds to induce apoptosis [181-185], and attenuates AR signaling by inhibiting AR nuclear localization [186]. Furthermore, silibinin also mediates MET in PC cells, as evidenced by reduced proliferation, adhesion, and migratory potential of PC cell lines [187], decreased expression of mesenchymal biomarkers [188], and upregulation of the epithelial biomarkers [189]. HIF-1 $\alpha$, which induces EMT in response to hypoxia via upregulation of TWIST1 [128], is also inhibited by silibinin [190].

Dietary consumption of another compound, Genistein, an isoflavone found in soy beans, is associated with a lower risk of PC and PC metastasis ([191] and references therein). Interestingly, Genistein treatment results in MET of PC cells, as evidenced by altered biomarker expression and decreased invasion [191]. Exposure of Genistein also reduces CD44+ cancer stem cells, inhibits the Hedgehog-Gli1 pathway [192], and upregulates miR-574-3p, which decreases proliferation, migration, and invasion of PC cells [193]. Pathway analysis indicates that miR-574-3p controls several genes involved in the Jak-STAT and Wnt signaling networks [193]. This suggests that a small molecule, Genistein, controls EP via miRmediated regulation of the Wnt and other signaling pathways.

Treatment with the proteasome inhibitor salinosporamide A (NPI-0052) also causes an MET in the mesenchymal-like DU-145 cells, with reduced levels of SNAIL and upregulation of E-cadherin. SNAIL repression is driven by inhibition of $\mathrm{NF}-\mathrm{KB}$ and upregulation of Raf kinase inhibitory protein (RKIP), a known inhibitor of metastasis [194]. RKIP expression in DU145 cells leads to reduced levels of SNAIL expression, whereas SNAIL overexpression in LNCaP cells antagonizes RKIP levels, leading to increased metastatic capacity. Moreover, treatment with a specific NF- $\mathrm{kB}$ inhibitor, dehydroxymethylepoxyquinomicin, mirrors the EMT repression that is observed upon treatment with salinosporamide A [194]. Together, these results implicate the proteasome as a potential modulator of EMT via a SNAIL/NF-kB/RKIP pathway.

\subsection{Stemness as a mediator of EP}

Work by the Weinberg laboratory and other groups has shown that EMT results in enrichment of cell populations with stem cell properties of self-renewal, clonogenic growth, and ability for differentiation in several cancer models $[10,195,196]$. In PC, CD44+ LNCaP and DU145 cells lose E-cadherin and are more invasive than their CD44- counterparts [197]. EMT has also been associated with the acquisition of a stem-like phenotype in PC-3 and $\mathrm{ARCaP}_{\mathrm{M}}$ cells [173]. Similarly, knockdown of the ETS transcription factor ESE/EHF in immortalized prostate epithelial cells leads to EMT, acquisition of stem- 
like properties, tumorigenic capability, and metastatic dissemination [198]. Association of cancer-associated fibroblasts with PC-3 cells also leads to EMT, along with upregulation of CD133 and an increase in CD $44^{\text {high }} / \mathrm{CD} 24^{\text {low }}$ cells, which display self-renewal capacity and tumorigenicity [14]. In PC model systems, overexpression of the polycomb repressor BMI-1 is required for de-differentiation, prostate stem cell renewal, and has been linked to malignant transformation [199]. In the clinical context, evidence for EP and stemness can be found in the examination of CTCs from men with CRPC. CTCs have been found to coexpress epithelial and mesenchymal markers, and $>80 \%$ of CTCs from CRPC patients also express the stemness marker CD133, suggesting that stemness may play a role in modulating EP during metastatic dissemination through the vasculature [15]. Finally, evidence is strong for the loss of epithelial biomarker expression during castration and chemotherapy-resistant progression in human PC, and this EP is linked to upregulation of stemness pathways including Hedgehog and NOTCH signaling, suggesting the importance of the dual regulation of EP by these embryonic programs [200].

There is, however, also evidence of PC cell lines in which cancer stem cells are enriched for an epithelial phenotype. ECadherin positive subpopulations of DU145 and PC-3 cells express embryonic stem cell markers SOX2, OCT3/4, Nanog, and c-Myc. Furthermore, the E-cadherin positive populations form tumors, while E-cadherin negative sublines do not [201]. Additionally, DU145 cells treated with chemotherapy generates drug-tolerant lines with low tumor initiating capacity, and addition of 5'-aza-deoxycytidine to drug-tolerant cells leads to re-expression of E-cadherin and $\mathrm{CD} 44+$, with increased tumorigenic potential [202]. Moreover, it has been shown that an epithelial-like subpopulation of PC-3 cells is enriched in tumor initiating cells (TICs) while the mesenchymal subpopulation are depleted in TICs [37]. Overexpression of SNAIL in the epithelial-like TICs reduces their self-renewal and metastatic capacity, concomitant with an EMT-like event [37]. Conversely, combined knockdown of SNAIL, ZEB1, and TWIST leads to an epithelial phenotype, enhanced spheroid formation, and self-renewal programs [37]. In a review of CSCs in PC, a distinction is made between TICs and CSCs, highlighting that the existence of TICs suggests the clonality of tumor cells rather than a hierarchical structure of the tumor [203]. Yet, despite this distinction, the data surrounding CSCs and EP highlight the dynamic and complex relationships between stem-like programs and EP pathways and suggest that EMT may not be the sole driver of PC cell tumorigenicity and invasive potential.

Based on these findings, we hypothesize that it is the ability to interconvert reversibly between epithelial and nonepithelial stem-like phenotypic states (plasticity) that drives metastatic spread and lethality in PC (and likely other solid tumors), rather than the epithelial or mesenchymal state in isolation.

\section{Evidence of EP in treatment-resistant and disseminated PC}

The above sections suggest a role for EP in the development of invasiveness, treatment resistance, and dissemination in PC model systems. Observing this plasticity in the clinic is a greater challenge given that EP is transient, may occur in rare cellular populations at the invasive edges of the tumor, and that the gold-standard biomarkers of EP in PC are still being defined. Furthermore, metastatic tissue in PC is not collected or analyzed routinely, metastatic tissue architecture and phenotype can be heterogeneous, and the ability to observe EP biomarkers in patients is likely context dependent. EP is linked to drug resistance [204], and there is emerging evidence that EP mediates resistance to local therapy (surgery or radiation), hormonal therapies, immunotherapies, and chemotherapeutics commonly used to treat PC. The following sections detail the clinical evidence to supporting a causal relationship between EP and treatment failure due to resistance in human PC. Selected clinical states of PC and their associations with EP are highlighted in Table 2.

\subsection{Detecting EP in PC}

One of the challenges in establishing the existence and relevance of EP in PC metastasis is the difficulty visualizing the process. To establish distant metastases, invasive cancer cells likely circulate in the bloodstream and settle in other organs, which in CRPC is often bone. Evidence supporting EP is found through an analysis of circulating tumor cells (CTCs). CELLSEARCH $^{\circledR}$ (Janssen/Veridex) is the only FDA-cleared technology for the detection of CTCs, which are defined as nucleated, cytokeratin-positive, and CD45-negative cells immunomagnetically captured from the bloodstream using antibodies against epithelial cell adhesion molecule [205]. CTCs can be enumerated to provide prognostic information in multiple tumor types [206-209], but more importantly, CTCs carry genotypic and phenotypic information about an individual's tumor at a discrete point in time. A substantial number (30-40 \%) of men with advanced metastatic CRPC do not have detectable CTCs using the CELLSEARCH ${ }^{\circledR}$ epithelial-based method [210], and recent evidence indicates that there is phenotypic heterogeneity among CTCs, with some CTCs expressing not only epithelial proteins but also mesenchymal and stemness proteins, indicators of EP [15]. We have found that a range of EP biomarkers are expressed in CRPC CTCs, including loss of E-cadherin and gain of Ncadherin, vimentin, CD133, $\beta$-catenin, and OB-cadherin. Importantly, many CTCs have a dual epithelial and mesenchymal/stemness phenotype, suggesting the importance of this duality in treatment resistance and dissemination [15]. This EP biology is not unique to PC, as variable phenotypes have been observed in CTCs from other malignancies, such as 
lung [211, 212], colorectal [213], and breast cancer [214], suggesting a broad conceptual parallel. Therefore, EP may explain the underdetection of CTCs in patients with advanced malignancy using the standard epithelial antigen-based technology $[15,215,216]$. There are a number of technologies under development that employ nonepithelial targets for CTC capture and characterization and may provide a noninvasive window into the role of EP in cancer metastasis [217].

Given its dynamic and transient nature, visualizing EP is a major challenge radiographically. EP may be routinely seen but not clinically recognized through tumor imaging. In PC, there is well-documented discordance between PSA measurements and imaging responses. For example, technetium-99 bone scans indirectly assess osteoblastic activity induced by PC metastases to bone, and the interpretation is often complicated by the "flare phenomenon," which is an osteoblastic reaction that may occur in response to treatment where new or increased intensity of existing lesions is noted. The flare gives the appearance of worsening of bony metastatic disease, but is not adversely prognostic. For instance, in a phase II study of abiraterone plus prednisone in patients with metastatic CRPC, over half of the patients responding to abiraterone by PSA criteria had initial worsening of the bone scan, but more than $80 \%$ of those scans improved subsequently, consistent with the flare phenomena [218]. We hypothesize that this initial flare may represent an element of EP induced by treatment, in which PC osteomimicry linked to induction of EMT becomes evident during the initial phases of treatment. During treatment-induced EMT, the mesenchymal, stem-like cells mimic osteoblasts and take up more technetium-99, accounting for these early changes on bone scans. Although this imaging flare temporarily stabilizes and often improves, the bone lesions typically progress at a later time point, indicating persistent viable tumor in these regions of bone scan activity. Given that a number of agents used to treat men with PC, such as hormonal therapies, can induce this reaction, and that osteomimicry markers may likewise emerge during ADT $[15,65]$, the bone scan flare may be imaging evidence of a shift toward a bone-forming mesenchymal state and thus plasticity.

In contrast to the flare phenomenon described above, in a phase II study of the c-met/VEGFR2 inhibitor cabozantinib in metastatic CRPC, nearly $80 \%$ of patients had complete or partial resolution of bone scan lesions after 12 weeks of therapy, but bone scan response did not correlate with PSA or CTC response [219]. The initial imaging improvement with cabozantinib is typically short-lived, with the re-emergence of active bone lesions over time in the same regions, indicating persistent viable tumor despite the disappearance on scans. We hypothesize that the changes visualized on bone scan during the course of treatment with cabozantinib may be the result of cellular plasticity and induction of MET. This induced MET would shift away from the osteoblastic mesenchymal state in bone metastases and toward a more epithelial, nonbone-forming state, and lead to a transient reduction in technetium-99 uptake. This may be accompanied by a rise in PSA due to this epithelial differentiation driven by AR activity [220], which is often disconnected from the radiographic changes. Thus, PSA changes reflecting epithelial biology and bone scan changes representing mesenchymal tumor biology may be clinical biomarkers of EP. Further studies to quantify these changes in the context of tumor biopsies during a range of therapies are needed.

\subsection{EP in localized PC}

Although advanced metastatic PC is known to be a heterogeneous disease [6], it has been demonstrated that most metastases arise from a single precursor lesion in the primary tumor, suggesting that lethal PC has a monoclonal origin [8]. Therefore, differences in phenotype rather than genotype must account for the heterogeneity, and even in localized PC, there is evidence for EP. For example, TWIST1 is absent in benign prostatic tissue but expressed in prostate adenocarcinoma cells, and higher levels of TWIST1 expression are associated with higher Gleason scores in the primary tumor [221]. By immunohistochemistry, higher expression of EMT markers can be seen at the invasive front of the tumor versus the center of the tumor. For example, E-cadherin expression decreases at the invasive front while vimentin and ZEB-1 expression increase [222]. Similarly, in the primary prostate tumor, the combination of weak E-cadherin and strong N-cadherin expression, or high vimentin or TWIST1 expression, predict early dissemination and clinical recurrence [223, 224].

A frequently observed genetic lesion in human PC is the TMPRSS2-ERG fusion, in which exon 1 of TMPRSS2, an androgen-regulated serine protease, is joined to exons 4-9 of the ERG gene, an erythroblast transformation-specific (ETS) transcription factor [225]. The fusion protein TMPRSS2-ERG is present in more than half of all PC [226]. Interestingly, TMPRSS2-ERG fusion can induce EMT via activation of the $\mathrm{Wnt} / \beta$-catenin pathway [227]. In addition, EMT can be induced in vitro and in vivo by overexpression of the TMPRSS2-ERG fusion. Here, EMT is mediated by ZEB1 and ZEB2, and chromatin immunoprecipitation assays revealed that TMPRSS2-ERG directly binds the ZEB1 promoter [228]. This suggests that the TMPRSS2-ERG fusion may be associated with more aggressive disease by controlling ZEB1-induced EMT and offers a biological explanation for the prognostic significance attributed to detection of the TMPRSS2-ERG protein. In a cohort of men with localized PC undergoing active surveillance, those men with the TMPRSS2-ERG fusion had a higher likelihood of PCspecific mortality [229]. Additional studies show that the presence of the fusion protein predicts for recurrence after prostatectomy [230] and portends a worse survival [231]. 
This is controversial, however, as a recent metaanalysis found no association between ERG overexpression via TMPRSS2ERG fusion and recurrence or mortality [232], and the relevance of the genomic rearrangement may be variant dependent. For example, one variant found in approximately $5 \%$ of PC is the TMPRSS2-ERG fusion together with the deletion of sequences 5' to ERG, and the presence of this variant confers a poor prognosis [231].

Radiation therapy is commonly used to treat localized PC and, in many men, is curative; however, greater than one third of men with high-risk disease will relapse after local radiotherapy. There is concern, however, that the emerging tumor clones in men who fail radiotherapy may undergo EMT and develop an associated treatment resistance. For example, ionizing radiation induces DNA double-strand breaks, and the DNA damage response can induce stromal cells to secrete WNT16B, a soluble protein that may induce EMT mediated through the NF-KB pathway in neighboring PC cells. WNT16B overexpression has been observed during cytotoxic chemotherapy and radiation in PC patients and model systems and has been recently linked to treatment failure and dissemination [150]. Thus, EP is emerging as an adaptive stressactivated mechanism of resistance to radiotherapy and cytotoxic therapy that is induced by stromal signaling.

\subsection{EP in metastatic PC}

Gene expression analysis of single CTCs revealed increased expression of EMT-related genes in CRPC patients compared to castrate-sensitive patients, suggesting that activation of EMT-related genes may be associated with disease progression [233]. For example, NOTCH-1, which has been associated with an EMT and stem cell phenotype [173], is significantly upregulated in bone metastasis compared with the primary prostate tumors, suggesting that NOTCH-1 may be important for PC progression [222].

As discussed above, EP is increasingly recognized as a mechanism underlying drug resistance, and in PC, evidence exists for the upregulation of mesenchymal biomarkers during androgen deprivation in cell lines, animal models, and in patient tumor specimens. For example, expression of the mesenchymal marker N-cadherin increases after androgen deprivation in men treated prior to surgery [234]. Furthermore, ADT has been shown to induce an EMT, possibly by removing the inhibitory effect that AR signaling has on the transcription factor, ZEB-1. However, these cells are able to revert to an epithelial phenotype upon replacement of testosterone, indicating EP [65]. N-Cadherin expression is rare in untreated PC, increases with androgen deprivation, and is highest in the castration-resistant setting [235]. In the primary prostate tumor, the combination of weak E-cadherin and strong N-cadherin expression predicts for early biochemical failure and clinical recurrence [223]. N-Cadherin expression has been associated with a more rapid progression to castration resistance, which may be circumvented preclinically through direct targeting with monoclonal antibodies to N-cadherin [235]. With this rationale, one could hypothesize that high $\mathrm{N}$-cadherin expression would predict for resistance to agents that block AR signaling; however, clinical studies are needed to confirm the role of mesenchymal biomarkers in predicting treatment resistance to pathways that target androgen synthesis or signaling.

Metastatic sites may variably express EP markers, and this variability may exist within and between patients. For example, in a metastatic survey study of human PC, lymph node metastases frequently had lower E-cadherin expression levels than bone metastases in the same patient [236]. This heterogeneity may reflect different modes of invasion or migration, such as collective sheet migration to lymph nodes, which may be independent of EP, as compared to a TGF- $\beta$-mediated hematogenous dissemination that has a greater requirement for EMT/MET [237]. In PC, metastatic site has prognostic importance, as lymph node metastatic CRPC has the most favorable prognosis, followed by bone-metastatic and visceral metastatic CRPC [238].

Docetaxel, an antimitotic microtubule-stabilizing agent, is the most commonly used chemotherapy for PC, and resistance to this agent often emerges within 6-12 months of treatment initiation. Recent evidence shows that PC cells lacking the epithelial marker cytokeratin (CK18 and CK19) are able to survive treatment with docetaxel. These docetaxel-resistant cells are more abundant in metastatic sites as compared to the primary tumor [200]. In cell line and xenograft models, docetaxel-resistant cells are induced by activation of stemness pathways important for EP and can be depleted by combining docetaxel with agents that target the NOTCH and Hedgehog signaling pathways [200]. Loss of CK or PSA in prostatectomy specimens is associated with recurrence and metastasis as well [239], suggesting that identification of cytokeratin- or PSA-negative PC cells may predict for resistance to local or systemic therapies, but additional validation is necessary. Given that taxanes have been shown to induce EP and stemness in several model systems, accompanied by treatment resistance and dissemination, therapies that reduce this resistance mechanism are needed [204].

EP may also lead to resistance to immunotherapy. Treatment with an epithelial-based complementary DNA (cDNA) vaccine results in regression of prostate tumors in mice, but when resistant tumors eventually emerge, these tumors lack PSA expression and gain mesenchymal markers. Revaccination with a cDNA library derived from the resistant tumors eradicates the tumors and cures the mice. Reversal of the vaccination strategy, giving the mesenchymal vaccine followed by the epithelial vaccine, is ineffective [240]. This is further evidence for the role of EP in treatment resistance and may provide clues as to how to tailor treatment to target these resistance pathways. 
Given that the FDA-approved PC immunotherapy sipuleucel-T and the investigational vaccine Prostvac utilizes epithelial differentiation proteins (prostatic acid phosphatase and PSA, respectively) to prime dendritic and $\mathrm{T}$ cells, and results in only modest survival benefits [241, 242], tumor escape from this immunotherapy may involve EP and loss of epithelial targets or upregulation of mesenchymal or stemness targets.

As discussed in the clinical vignette above, one path to CRPC may be through neuroendocrine differentiation (NED), in which PC cells no longer secrete PSA or express AR. Instead, the cells often express and secrete chromogranin A [20], and this may be another example of EP. Clinically, NED most often occurs after ADT or AR signaling inhibition. Likewise, preclinically, depletion of androgen in cell culture promotes NED of PC cells [243], and NED in response to androgen deprivation in cell lines is dependent on Akt activity [62]. Given the known crosstalk between the AR and PI3KAkt pathways [244], there is rationale for a combination approach clinically, and PI3K-Akt pathway inhibitors are currently under investigation both as single agents and in combination (reviewed in [245]). PC tumors with NED often have high levels of EZH2, which as discussed above, leads to suppression of DAP2IP and subsequent activation of important oncogenic pathways and EMT, further supporting the hypothesis that NED is a result of EP [28]. To further classify NED in PC, next-generation RNA sequencing was performed on primary tumors and metastatic biopsy samples from men with NED and compared with tumors from men with classic prostate adenocarcinoma. Aurora kinase A and N-myc are overexpressed and amplified in $40 \%$ of NED versus $5 \%$ of classic prostate adenocarcinoma and cooperate to induce NED in prostate cells [28]. This suggests that aurora kinase inhibitors may be used alone or in combination with cytotoxic chemotherapy to treat NED in PC, and trials targeting aurora kinase A are ongoing. Finally, whether NED is associated with EP or genetic evolution in PC is not clear. However, small cell differentiation of nonsmall cell lung carcinomas has been reported during EGFR inhibition, which is reversible phenotypically upon withdrawal of the epithelial targeting agent [22]. This suggests that a similar phenomenon may be occurring in PC during ADT or with potent AR inhibition.

\section{Therapeutic strategies directed toward EP}

As described above, there is substantial evidence that one mechanism of drug resistance is through phenotypic plasticity. In the era of personalized medicine, combination anticancer therapies have fallen somewhat out of favor; however, rational combination approaches may eradicate PC, similar to the way combination therapy revolutionized treatment for leukemia and infectious diseases, such as tuberculosis and HIV/AIDS.
Combination strategies in preclinical models of malignancy have turned cytostatic activity into cytocidal activity and resulted in durable remissions. Therefore, combining therapies based on the knowledge of resistance pathways inherent to the cancer cell and the tumor microenvironment is an emerging and essential step in oncology [246]. We hypothesize that targeting the underlying regulators of EP, such as stemness pathways, epigenetic regulators, or oncogenic pathways, will be more effective than single agent therapies directed against more traditional epithelial differentiation pathways. These epithelial differentiation pathways, including AR and androgen synthesis, are not likely to be causally related to PC survival, given the inevitable resistant escape observed clinically after relatively short intervals of time. While a PC stem cell has not been clearly identified, it is possible that AR is not present in this stem-like cell [247], and thus, strategies to target key stemness, invasion, and dissemination pathways may be of greater benefit than AR targeting. However, given the central role of $\mathrm{AR}$ in $\mathrm{PC}$ and its potential role in promoting survival of $\mathrm{PC}$ cells, targeting $\mathrm{AR}$ in the context of additional therapies directed against EP regulators may remain critical. Indeed, multiple pathways may require targeting in order to address the bulk of the differentiated cancer and its stem-like progeny [200].

There are several available drugs and therapies in development that specifically target the epithelial or the mesenchymal phenotype or stemness pathways, and potential therapeutic approaches to addressing EP in the clinic are listed in Table 3. Agents directed toward epithelial targets, such as androgen synthesis and AR signaling inhibitors, may need to be partnered with therapy against mesenchymal targets for maximal benefit. For example, in cell lines with constitutively active AR variants, there is increased expression of mesenchymal markers including $\mathrm{N}$-cadherin, again implicating EP as a mechanism of treatment resistance [248]. Furthermore,there is a monoclonal antibody against $\mathrm{N}$-cadherin that, in mouse models, prevents invasion and metastasis and delays the time to castration-resistance [236]. Combining a pure mesenchymal target such as this with an epithelial target may be a rational approach, such as combinations with enzalutamide or abiraterone acetate. Epithelial-antigen immunotherapies such as Prostvac (against PSA) or sipuleucel-T (against PAP) may lead to mesenchymal or stemness-based immune escape, similar to what has been observed preclinically, and thus novel targeting of mesenchymal or stemness antigens may be more productive long term. In addition, targeting of stromal cells directly through prodrugs, monoclonal antibodies, or chemokine inhibitors may reduce EP and invasion indirectly [249].

Approaches that target embryologic pathways important in regulating EP may provide clinical benefits similar to those observed preclinically. For example, treatment with a cytotoxic agent such as docetaxel may reduce the bulk of disease, but disease relapse is inevitable. Activation of Hedgehog or 
Table 3 Potential therapeutic strategies directed toward EP

\begin{tabular}{|c|c|c|c|}
\hline Therapy & Mechanism of action & Efficacy in human PC & References \\
\hline \multicolumn{4}{|l|}{ Epithelial phenotypic targets } \\
\hline \multicolumn{4}{|l|}{ Androgen receptor antagonist } \\
\hline $\begin{array}{l}\text { Enzalutamide } \\
\text { ARN-509 }\end{array}$ & Blocks AR, targets epithelial cells & $\begin{array}{l}\text { Enzalutamide prolongs survival; } \\
\text { Multiple agents in phase II-III trials }\end{array}$ & {$[256-258]$} \\
\hline \multicolumn{4}{|l|}{ TOK-001 } \\
\hline \multicolumn{4}{|l|}{ Androgen synthesis inhibitors } \\
\hline $\begin{array}{l}\text { Abiraterone } \\
\text { Orteronel }\end{array}$ & $\begin{array}{l}\text { Inhibits the CYP17 enzymes needed for } \\
\text { testosterone synthesis, targets epithelial cells }\end{array}$ & $\begin{array}{l}\text { Abiraterone prolongs survival; } \\
\text { orteronel in phase II-III trials }\end{array}$ & {$[258-261]$} \\
\hline \multicolumn{4}{|l|}{ TOK-001 } \\
\hline \multicolumn{4}{|l|}{ Mesenchymal phenotypic targets } \\
\hline \multicolumn{4}{|l|}{ N-Cadherin } \\
\hline $\begin{array}{l}\text { Anti-N-cadherin antibody } \\
\text { ADH-1 (Exherin) }\end{array}$ & $\begin{array}{l}\text { Block N-cadherin to slow tumor growth and } \\
\text { inhibit EMT }\end{array}$ & Unknown & {$[235,262]$} \\
\hline \multicolumn{4}{|l|}{ Clusterin inhibition } \\
\hline OGX-011 (custersin) & $\begin{array}{l}\text { Antisense oligonucleotide against secretory } \\
\text { clusterin, may inhibit EMT }\end{array}$ & $\begin{array}{l}\text { OGX-011 in combination with } \\
\text { docetaxel improved survival in a } \\
\text { phase II of men with CRPC }\end{array}$ & {$[92,93]$} \\
\hline \multicolumn{4}{|l|}{ C-met } \\
\hline Cabozantinib & $\begin{array}{l}\text { Tyrosine kinase inhibitor against MET and } \\
\text { VEGFR2 }\end{array}$ & $\begin{array}{l}\text { Bone scan and progression-free } \\
\text { survival improvement }\end{array}$ & [219] \\
\hline Sarasinoside A1 & Induces MET, even in the absence of E-cadherin & Unknown & {$[263]$} \\
\hline \multicolumn{4}{|l|}{ Stromal targets } \\
\hline fibroblast specific protein (FSP) & Prodrug targets stroma and may prevent EMT & Unknown & [249] \\
\hline FGFR family (mesenchymal isoforms) & Inhibits invasion, survival & Unknown & {$[264]$} \\
\hline Aurora kinase A inhibitor (MLN8237) & Blocks neuroendocrine differentiation & MLN8237 in phase II trials & {$[28]$} \\
\hline \multicolumn{4}{|l|}{ Combination approaches } \\
\hline \multicolumn{4}{|l|}{ Immunologic therapies in combination } \\
\hline Checkpoint/vaccine strategies & $\begin{array}{l}\text { Target multiple antigens during escape from } \\
\text { initial immunotherapy }\end{array}$ & Unknown & [240] \\
\hline \multicolumn{4}{|l|}{ Epigenetic therapies in combination } \\
\hline HDAC inhibitors & Induce EMT or MET & Unknown & {$[156]$} \\
\hline \multicolumn{4}{|l|}{ Stemness pathway targets } \\
\hline TGF- $\beta$ pathway inhibitors & $\begin{array}{l}\text { Kinase inhibition, neutralizing antibodies, } \\
\text { or antisense oligonucleotides }\end{array}$ & Unknown & {$[252]$} \\
\hline Hedgehog/Gli signaling inhibitors & Small molecule inhibition of Gli & GDC-0449 in phase 1-2 trials & {$[248]$} \\
\hline NOTCH inhibitors & Gamma secretase inhibition & Unknown, ongoing & {$[251]$} \\
\hline PI3K/PTEN pathway inhibitors & Reduced stemness, survival & BKM120, others in phase $1-2$ trials & {$[245]$} \\
\hline
\end{tabular}

$P S A$ prostate specific antigen, $A D T$ androgen deprivation therapy, $C R P C$ castration-resistant prostate cancer

NOTCH signaling in CRPC patients suggests that biomarkers of stemness may predict for benefit of agents that block stemness pathways. Hedgehog and NOTCH signaling inhibition is an active area of investigation in prostate and other cancers, and clinical trials with these agents alone and in combination are ongoing (reviewed in [250, 251]). Combination therapy with Hedgehog or NOTCH inhibition to address the stem-like cells with loss of epithelial differentiation may be more effective than treatment with either agent alone [200]. However, investigation of the selectivity of these agents against tumor cells rather than normal hematopoietic and organ-specific stem cell niches will be imperative given the potential for stem-cell toxicity. In a high-throughput drug screen to uncover agents specific to EMT-induced stemness properties, there were only a handful of agents, such as salinomycin, that were specifically toxic to cancer stem cells over normal cells, illustrating the formidable problem of selectivity. In this screen, paclitaxel actually induced a greater metastatic burden and promoted stemness properties [204]. These surprising findings require further 
validation in PC model systems, where new classes of agents more specific to the underlying biology of EP rather than differentiated cells may bear greater fruit.

Another stemness target under investigation is TGF- $\beta$ and the differing roles of TGF- $\beta$ in early versus late stage cancer and in mediating hematogenous versus lymph node metastases, as described above, highlights the need for biomarkers to help guide patient selection for treatment with these agents. Clinical trials with anti-TGF- $\beta$ therapies will likely show different results depending on the clinical context and again may be more effective when given in combination (reviewed in [252]). A clearly defined biomarker or set of biomarkers for EP in PC is needed to track these phenotypically diverse cells as they progress and contribute to treatment resistance. For example, detection of AR variants may be predictive of treatment response or resistance [253]. As reviewed elsewhere, predictive biomarkers in CRPC require extensive validation and prospective qualification both preclinically and in clinical trials, before they can be incorporated into clinical practice [254]. AR-independent PC may also be important in the development of EP, and identifying biomarkers of the different PC disease states and their relationship with EP is crucial.

Finally, because disease stability and differentiation rather than rapid cytoreduction and tumor shrinkage may occur with these therapies, especially when investigated as single agents, clinical trial endpoints that adequately test the activity of antiplasticity or stemness agents are necessary. In CRPC, these endpoints may include metastasis-free survival, overall survival, and radiographic or clinical progression-free survival. Combination approaches leading to novel cure model based clinical trial designs would also provide fair tests of substantial long term activity while limiting sample size [255], similar to what has been observed in the treatment of tuberculosis and HIV infections. Thus, combination approaches of EP targeted therapy with more traditional hormonal, immunomodulatory, or chemotherapies may extend survival, similar to what has been observed preclinically.

\section{Conclusions}

Substantial improvements in outcomes have been realized with novel hormonal therapies used for the treatment of metastatic CRPC, including abiraterone acetate and enzalutamide, and with immunotherapies and chemotherapies, such as sipuleucel-T, docetaxel, and cabazitaxel. Despite these incremental advances, treatment resistance emerges within 12 years in most cases, suggesting that novel approaches are needed. With the clinical use of more potent androgen pathway inhibitors, the emergence of neuroendocrine and other variant phenotypes is predicted to rise. EP is clearly associated with dissemination in multiple solid tumors, and emerging evidence supports EP as a mediator of both hematogenous dissemination (bone, visceral metastases) and therapeutic failure. To address this biology, novel agents that target stemness and embryonic pathways that influence cellular differentiation and invasion will be needed, likely in combination with current therapies that target the more differentiated epithelial bulk of the metastatic lesions. Rational combination therapies, based on the knowledge of feedback resistance pathways inherent to the cancer cell and tumor microenvironment, as well as on knowledge of immunologic escape due to loss of epithelial antigens, will likely be the most effective way to target EP in PC.

\section{Key unanswered questions}

1 How is AR regulation related to EP in PC and are these two pathways linked?

2 Can metastasis occur in human PC without loss of an epithelial phenotype or gain of a mesenchymal phenotype? Can other forms of migration/invasion, such as amoeboid invasion or collective sheet migration also explain dissemination and treatment failure?

3 Does EP explain treatment resistance to enzalutamide and abiraterone acetate or immunotherapy with sipuleucel-T based on studies of CTCs and metastatic biopsies over time in patients?

4 Can combination approaches targeting both epithelial and stem-like/mesenchymal compartments lead to eradication of established metastases or are these approaches more effective at preventing metastatic disease?

5 Does secondary neuroendocrine PC emerge due to genetic evolution and clonal selection over time or can this phenotype be reversed through systemic therapies, implying cellular plasticity?

Acknowledgments We acknowledge grant support from NIGMS grant R01 GM63090 (M.A. Garcia-Blanco); National Cancer Institute grant R01 CA127727, (M.A. Garcia-Blanco), Robert B. Goergen Prostate Cancer Foundation Young Investigator Award (A.J. Armstrong), Department of Defense Physician Research Training Award W81XWH-10-10483 (A.J. Armstrong).

\section{References}

1. Siegel, R., Naishadham, D., \& Jemal, A. (2012). Cancer statistics, 2012. CA: A Cancer Journal for Clinicians, 62, 10-29.

2. Rini, B. I., \& Small, E. J. (2002). Hormone-refractory prostate cancer. Current Treatment Options in Oncology, 3, 437-446.

3. Chen, C. D., Welsbie, D. S., Tran, C., Baek, S. H., Chen, R., Vessella, R., Rosenfeld, M. G., \& Sawyers, C. L. (2004). Molecular determinants of resistance to antiandrogen therapy. Nature Medicine, 10, 33 39.

4. Dehm, S. M., Schmidt, L. J., Heemers, H. V., Vessella, R. L., \& Tindall, D. J. (2008). Splicing of a novel androgen receptor exon 
generates a constitutively active androgen receptor that mediates prostate cancer therapy resistance. Cancer Research, 68, 5469-5477.

5. Mostaghel, E. A., Page, S. T., Lin, D. W., Fazli, L., Coleman, I. M., True, L. D., Knudsen, B., Hess, D. L., Nelson, C. C., Matsumoto, A. M., Bremner, W. J., Gleave, M. E., \& Nelson, P. S. (2007). Intraprostatic androgens and androgen-regulated gene expression persist after testosterone suppression: therapeutic implications for castration-resistant prostate cancer. Cancer Research, 67, 5033-5041.

6. Shah, R. B., Mehra, R., Chinnaiyan, A. M., Shen, R., Ghosh, D., Zhou, M., Macvicar, G. R., Varambally, S., Harwood, J., Bismar, T. A., Kim, R., Rubin, M. A., \& Pienta, K. J. (2004). Androgenindependent prostate cancer is a heterogeneous group of diseases: Lessons from a rapid autopsy program. Cancer Research, 64, 9209 9216.

7. Rubin, M. A., Putzi, M., Mucci, N., Smith, D. C., Wojno, K., Korenchuk, S., \& Pienta, K. J. (2000). Rapid ("warm") autopsy study for procurement of metastatic prostate cancer. Clinical Cancer Research, 6, 1038-1045.

8. Liu, W., Laitinen, S., Khan, S., Vihinen, M., Kowalski, J., Yu, G., Chen, L., Ewing, C. M., Eisenberger, M. A., Carducci, M. A., Nelson, W. G., Yegnasubramanian, S., Luo, J., Wang, Y., Xu, J., Isaacs, W. B., Visakorpi, T., \& Bova, G. S. (2009). Copy number analysis indicates monoclonal origin of lethal metastatic prostate cancer. Nature Medicine, 15, 559-565.

9. Aryee, M. J., Liu, W., Engelmann, J. C., Nuhn, P., Gurel, M., Haffner, M. C., Esopi, D., Irizarry, R. A., Getzenberg, R. H., Nelson, W. G., Luo, J., Xu, J., Isaacs, W. B., Bova, G. S., \& Yegnasubramanian, S. (2013). "DNA methylation alterations exhibit intraindividual stability and interindividual heterogeneity in prostate cancer metastases,". Science Translational Medicine, 5, $169 \mathrm{ra} 10$.

10. Mani, S. A., Guo, W., Liao, M. J., Eaton, E. N., Ayyanan, A., Zhou, A. Y., Brooks, M., Reinhard, F., Zhang, C. C., Shipitsin, M., Campbell, L. L., Polyak, K., Brisken, C., Yang, J., \& Weinberg, R. A. (2008). The epithelial-mesenchymal transition generates cells with properties of stem cells. Cell, 133, 704-715.

11. Thiery, J. P., \& Sleeman, J. P. (2006). Complex networks orchestrate epithelial-mesenchymal transitions. Nature Reviews Molecular Cell Biology, 7, 131-142.

12. Blick, T., Widodo, E., Hugo, H., Waltham, M., Lenburg, M. E., Neve, R. M., \& Thompson, E. W. (2008). Epithelial mesenchymal transition traits in human breast cancer cell lines. Clinical \& Experimental Metastasis, 25, 629-642.

13. Hugo, H., Ackland, M. L., Blick, T., Lawrence, M. G., Clements, J. A., Williams, E. D., \& Thompson, E. W. (2007). Epithelial-mesenchymal and mesenchymal-epithelial transitions in carcinoma progression. Journal of Cellular Physiology, 213, 374-383.

14. Giannoni, E., Bianchini, F., Masieri, L., Serni, S., Torre, E., Calorini, L., \& Chiarugi, P. (2010). Reciprocal activation of prostate cancer cells and cancer-associated fibroblasts stimulates epithelial-mesenchymal transition and cancer stemness. Cancer Research, 70, 69456956.

15. Armstrong, A. J., Marengo, M. S., Oltean, S., Kemeny, G., Bitting, R. L., Turnbull, J. D., Herold, C. I., Marcom, P. K., George, D. J., \& Garcia-Blanco, M. A. (2011). Circulating tumor cells from patients with advanced prostate and breast cancer display both epithelial and mesenchymal markers. Molecular Cancer Research, 9, 997-1007.

16. Zhu, M. L., \& Kyprianou, N. (2010). Role of androgens and the androgen receptor in epithelial-mesenchymal transition and invasion of prostate cancer cells. FASEB Journal, 24, 769-777.

17. Li, X., Lewis, M. T., Huang, J., Gutierrez, C., Osborne, C. K., Wu, M. F., Hilsenbeck, S. G., Pavlick, A., Zhang, X., Chamness, G. C., Wong, H., Rosen, J., \& Chang, J. C. (2008). Intrinsic resistance of tumorigenic breast cancer cells to chemotherapy. Journal of the National Cancer Institute, 100, 672-679.
18. Abraham, B. K., Fritz, P., McClellan, M., Hauptvogel, P., Athelogou, M., \& Brauch, H. (2005). Prevalence of CD44+/ CD24-/low cells in breast cancer may not be associated with clinical outcome but may favor distant metastasis. Clinical Cancer Research, 11, 1154-1159.

19. Ginestier, C., Hur, M. H., Charafe-Jauffret, E., Monville, F., Dutcher, J., Brown, M., Jacquemier, J., Viens, P., Kleer, C. G., Liu, S., Schott, A., Hayes, D., Birnbaum, D., Wicha, M. S., \& Dontu, G. (2007). ALDH1 is a marker of normal and malignant human mammary stem cells and a predictor of poor clinical outcome. Cell Stem Cell, 1, 555-567.

20. Taplin, M. E., George, D. J., Halabi, S., Sanford, B., Febbo, P. G., Hennessy, K. T., Mihos, C. G., Vogelzang, N. J., Small, E. J., \& Kantoff, P. W. (2005). Prognostic significance of plasma chromogranin a levels in patients with hormone-refractory prostate cancer treated in Cancer and Leukemia Group B 9480 study. Urology, 66, 386-391.

21. Aparicio, A. M., Harzstark, A., Corn, P. G., Wen, S., Araujo, J., Tu, S. M., Pagliaro, L., Kim, J., Millikan, R. E., Ryan, C. J., Tannir, N. M., Zurita, A., Mathew, P., Arap, W., Troncoso, P., Thall, P., \& Logothetis, C. J. (2013). Platinum-based chemotherapy for variant castrate-resistant prostate cancer. Clin Cancer Res, 19, 3621-3630.

22. Sequist, L. V., Waltman, B. A., Dias-Santagata, D., Digumarthy, S., Turke, A. B., Fidias, P., Bergethon, K., Shaw, A. T., Gettinger, S., Cosper, A. K., Akhavanfard, S., Heist, R. S., Temel, J., Christensen, J. G., Wain, J. C., Lynch, T. J., Vernovsky, K., Mark, E. J., Lanuti, M., Iafrate, A. J., Mino-Kenudson, M., \& Engelman, J. A. (2011). Genotypic and histological evolution of lung cancers acquiring resistance to EGFR inhibitors. Science Translational Medicine, 3, 75ra26.

23. Parwani, A. V., Kronz, J. D., Genega, E. M., Gaudin, P., Chang, S., \& Epstein, J. I. (2004). Prostate carcinoma with squamous differentiation: An analysis of 33 cases. The American Journal of Surgical Pathology, 28, 651-657.

24. di Sant'Agnese, P. A. (2001). Neuroendocrine differentiation in prostatic carcinoma: An update on recent developments. Annals of Oncology, 12(Suppl 2), S135-S140.

25. Berruti, A., Mosca, A., Tucci, M., Terrone, C., Torta, M., Tarabuzzi, R., Russo, L., Cracco, C., Bollito, E., Scarpa, R. M., Angeli, A., \& Dogliotti, L. (2005). Independent prognostic role of circulating chromogranin A in prostate cancer patients with hormonerefractory disease. Endocrine-Related Cancer, 12, 109-117.

26. Wang, W., \& Epstein, J. I. (2008). Small cell carcinoma of the prostate. A morphologic and immunohistochemical study of 95 cases. The American Journal of Surgical Pathology, 32, 65-71.

27. Kalluri, R., \& Weinberg, R. A. (2009). The basics of epithelialmesenchymal transition. Journal of Clinical Investigation, 119, 1420-1428.

28. Beltran, H., Rickman, D. S., Park, K., Chae, S. S., Sboner, A., MacDonald, T. Y., Wang, Y., Sheikh, K. L., Terry, S., Tagawa, S. T., Dhir, R., Nelson, J. B., de la Taille, A., Allory, Y., Gerstein, M. B., Perner, S., Pienta, K. J., Chinnaiyan, A. M., Collins, C. C., Gleave, M. E., Demichelis, F., Nanus, D. M., \& Rubin, M. A. (2011). Molecular characterization of neuroendocrine prostate cancer and identification of new drug targets. Cancer Discovery, 1, 487-495.

29. Giannoni, E., Taddei, M. L., Parri, M., Bianchini, F., Santosuosso, M., Grifantini, R., Fibbi, G., Mazzanti, B., Calorini, L., \& Chiarugi, P. (2013). EphA2-mediated mesenchymal-amoeboid transition induced by endothelial progenitor cells enhances metastatic spread due to cancer-associated fibroblasts. Journal Molecules Medical (Berl), 91, 103-115.

30. Koeneman, K. S., Yeung, F., \& Chung, L. W. (1999). Osteomimetic properties of prostate cancer cells: A hypothesis supporting the predilection of prostate cancer metastasis and growth in the bone environment. Prostate, 39, 246-261. 
31. Josson, S., Nomura, T., Lin, J. T., Huang, W. C., Wu, D., Zhau, H. E., Zayzafoon, M., Weizmann, M. N., Gururajan, M., \& Chung, L. W. (2011). beta2-microglobulin induces epithelial to mesenchymal transition and confers cancer lethality and bone metastasis in human cancer cells. Cancer Research, 71, 2600-2610.

32. Zhau, H. E., He, H., Wang, C. Y., Zayzafoon, M., Morrissey, C., Vessella, R. L., Marshall, F. F., Chung, L. W., \& Wang, R. (2011). Human prostate cancer harbors the stem cell properties of bone marrow mesenchymal stem cells. Clinical Cancer Research, 17, 2159-2169.

33. Yang, J., Fizazi, K., Peleg, S., Sikes, C. R., Raymond, A. K., Jamal, N., Hu, M., Olive, M., Martinez, L. A., Wood, C. G., Logothetis, C. J., Karsenty, G., \& Navone, N. M. (2001). Prostate cancer cells induce osteoblast differentiation through a Cbfa1-dependent pathway. Cancer Research, 61, 5652-5659.

34. Zhau, H. Y., Chang, S. M., Chen, B. Q., Wang, Y., Zhang, H., Kao, C., Sang, Q. A., Pathak, S. J., \& Chung, L. W. (1996). Androgenrepressed phenotype in human prostate cancer. Proceedings of the National Academy of Sciences of the United States of America, 93, 15152-15157.

35. Ke, X. S., Qu, Y., Goldfinger, N., Rostad, K., Hovland, R., Akslen, L. A., Rotter, V., Oyan, A. M., \& Kalland, K. H. (2008). Epithelial to mesenchymal transition of a primary prostate cell line with switches of cell adhesion modules but without malignant transformation. PLoS One, 3, e3368.

36. Marian, C. O., Yang, L., Zou, Y. S., Gore, C., Pong, R. C., Shay, J. W., Kabbani, W., Hsieh, J. T., \& Raj, G. V. (2011). Evidence of epithelial to mesenchymal transition associated with increased tumorigenic potential in an immortalized normal prostate epithelial cell line. Prostate, 71, 626-636.

37. Celia-Terrassa, T., Meca-Cortes, O., Mateo, F., de Paz, A. M., Rubio, N., Arnal-Estape, A., Ell, B. J., Bermudo, R., Diaz, A., Guerra-Rebollo, M., Lozano, J. J., Estaras, C., Ulloa, C., Alvarez-Simon, D., Mila, J., Vilella, R., Paciucci, R., Martinez-Balbas, M., de Herreros, A. G., Gomis, R. R., Kang, Y., Blanco, J., Fernandez, P. L., \& Thomson, T. M. (2012). Epithelial-mesenchymal transition can suppress major attributes of human epithelial tumor-initiating cells. The Journal of Clinical Investigation, 122, 1849-1868.

38. Moody, S. E., Perez, D., Pan, T. C., Sarkisian, C. J., Portocarrero, C. P., Sterner, C. J., Notorfrancesco, K. L., Cardiff, R. D., \& Chodosh, L. A. (2005). The transcriptional repressor Snail promotes mammary tumor recurrence. Cancer Cell, 8, 197-209.

39. Fan, F., Samuel, S., Evans, K. W., Lu, J., Xia, L., Zhou, Y., Sceusi, E., Tozzi, F., Ye, X. C., Mani, S. A., \& Ellis, L. M. (2012). Overexpression of Snail induces epithelial-mesenchymal transition and a cancer stem cell-like phenotype in human colorectal cancer cells. Cancer Medical, 1, 5-16.

40. Emadi Baygi, M., Soheili, Z. S., Schmitz, I., Sameie, S., \& Schulz, W. A. (2010). Snail regulates cell survival and inhibits cellular senescence in human metastatic prostate cancer cell lines. Cell Biology and Toxicology, 26, 553-567.

41. McKeithen, D., Graham, T., Chung, L. W., \& Odero-Marah, V. (2010). Snail transcription factor regulates neuroendocrine differentiation in LNCaP prostate cancer cells. Prostate, 70, 982-992.

42. Emadi Baygi, M., Soheili, Z. S., Essmann, F., Deezagi, A., Engers, R., Goering, W., \& Schulz, W. A. (2010). Slug/SNAI2 regulates cell proliferation and invasiveness of metastatic prostate cancer cell lines. Tumour Biology, 31, 297-307.

43. Wu, K., Gore, C., Yang, L., Fazli, L., Gleave, M., Pong, R. C., Xiao, G., Zhang, L., Yun, E. J., Tseng, S. F., Kapur, P., He, D., \& Hsieh, J. T. (2012). Slug, a unique androgen-regulated transcription factor, coordinates androgen receptor to facilitate castration resistance in prostate cancer. Molecular Endocrinology, 26, 1496-1507.

44. Graham, T. R., Zhau, H. E., Odero-Marah, V. A., Osunkoya, A. O., Kimbro, K. S., Tighiouart, M., Liu, T., Simons, J. W., \& O'Regan,
R. M. (2008). Insulin-like growth factor-I-dependent up-regulation of ZEB1 drives epithelial-to-mesenchymal transition in human prostate cancer cells. Cancer Research, 68, 2479-2488.

45. Yang, J., Mani, S. A., Donaher, J. L., Ramaswamy, S., Itzykson, R. A., Come, C., Savagner, P., Gitelman, I., Richardson, A., \& Weinberg, R. A. (2004). Twist, a master regulator of morphogenesis, plays an essential role in tumor metastasis. Cell, 117, 927-939.

46. Liu, A. N., Zhu, Z. H., Chang, S. J., \& Hang, X. S. (2012). Twist expression associated with the epithelial-mesenchymal transition in gastric cancer. Molecular and Cellular Biochemistry, 367, 195-203.

47. Yang, M. H., Hsu, D. S., Wang, H. W., Wang, H. J., Lan, H. Y., Yang, W. H., Huang, C. H., Kao, S. Y., Tzeng, C. H., Tai, S. K., Chang, S. Y., Lee, O. K., \& Wu, K. J. (2010). Bmil is essential in Twist1-induced epithelial-mesenchymal transition. Nature Cell Biology, 12, 982-992.

48. Eckert, M. A., Lwin, T. M., Chang, A. T., Kim, J., Danis, E., Ohno-Machado, L., \& Yang, J. (2011). Twistl-induced invadopodia formation promotes tumor metastasis. Cancer Cell, 19, 372-386.

49. Peinado, H., Olmeda, D., \& Cano, A. (2007). Snail, Zeb and bHLH factors in tumour progression: An alliance against the epithelial phenotype? Nature Reviews Cancer, 7, 415-428.

50. Watson, M. A., Ylagan, L. R., Trinkaus, K. M., Gillanders, W. E., Naughton, M. J., Weilbaecher, K. N., Fleming, T. P., \& Aft, R. L. (2007). Isolation and molecular profiling of bone marrow micrometastases identifies TWIST1 as a marker of early tumor relapse in breast cancer patients. Clinical Cancer Research, 13, 5001-5009.

51. Alexander, N. R., Tran, N. L., Rekapally, H., Summers, C. E., Glackin, C., \& Heimark, R. L. (2006). N-Cadherin gene expression in prostate carcinoma is modulated by integrin-dependent nuclear translocation of Twist1. Cancer Research, 66, 3365-3369.

52. Fridman, J. S., \& Lowe, S. W. (2003). Control of apoptosis by p53. Oncogene, 22, 9030-9040.

53. Vousden, K. H. (2000). p53: Death star. Cell, 103, 691-694.

54. Sigal, A., \& Rotter, V. (2000). Oncogenic mutations of the p53 tumor suppressor: the demons of the guardian of the genome. Cancer Research, 60, 6788-6793.

55. Chang, C. J., Chao, C. H., Xia, W., Yang, J. Y., Xiong, Y., Li, C. W., Yu, W. H., Rehman, S. K., Hsu, J. L., Lee, H. H., Liu, M., Chen, C. T., Yu, D., \& Hung, M. C. (2011). p53 regulates epithelial-mesenchymal transition and stem cell properties through modulating miRNAs. Nature Cell Biology, 13, 317-323.

56. Perk, J., Iavarone, A., \& Benezra, R. (2005). Id family of helixloop-helix proteins in cancer. Nature Reviews Cancer, 5, 603-614.

57. Ouyang, X. S., Wang, X., Lee, D. T., Tsao, S. W., \& Wong, Y. C. (2002). Over expression of ID-1 in prostate cancer. The Journal of Urology, 167, 2598-2602.

58. Zhang, X., Ling, M. T., Wang, Q., Lau, C. K., Leung, S. C., Lee, T. K., Cheung, A. L., Wong, Y. C., \& Wang, X. (2007). Identification of a novel inhibitor of differentiation-1 (ID-1) binding partner, caveolin-1, and its role in epithelial-mesenchymal transition and resistance to apoptosis in prostate cancer cells. The Journal of Biological Chemistry, 282, 33284-33294.

59. Williams, T. M., \& Lisanti, M. P. (2005). Caveolin-1 in oncogenic transformation, cancer, and metastasis. American Journal of Physiology. Cell Physiology, 288, C494-C506.

60. Yang, G., Truong, L. D., Wheeler, T. M., \& Thompson, T. C. (1999). Caveolin-1 expression in clinically confined human prostate cancer: A novel prognostic marker. Cancer Research, 59, 5719-5723.

61. Li, L., Ren, C. H., Tahir, S. A., Ren, C., \& Thompson, T. C. (2003). Caveolin-1 maintains activated Akt in prostate cancer cells through scaffolding domain binding site interactions with and inhibition of serine/threonine protein phosphatases PP1 and PP2A. Molecular and Cellular Biology, 23, 9389-9404. 
62. Wu, C., \& Huang, J. (2007). Phosphatidylinositol 3-kinase-AKTmammalian target of rapamycin pathway is essential for neuroendocrine differentiation of prostate cancer. The Journal of Biological Chemistry, 282, 3571-3583.

63. Ciarlo, M., Benelli, R., Barbieri, O., Minghelli, S., Barboro, P., Balbi, C., \& Ferrari, N. (2012). Regulation of neuroendocrine differentiation by AKT/hnRNPK/AR/beta-catenin signaling in prostate cancer cells. International Journal of Cancer, 131, 582-590.

64. Murthy, S., Wu, M., Bai, V. U., Hou, Z., Menon, M., Barrack, E. R., Kim, S. H., \& Reddy, G. P. (2013). Role of androgen receptor in progression of LNCaP prostate cancer cells from G1 to $\mathrm{S}$ phase. PLoS One, 8, e56692.

65. Sun, Y., Wang, B. E., Leong, K. G., Yue, P., Li, L., Jhunjhunwala, S., Chen, D., Seo, K., Modrusan, Z., Gao, W. Q., Settleman, J., \& Johnson, L. (2012). Androgen deprivation causes epithelial-mesenchymal transition in the prostate: Implications for androgendeprivation therapy. Cancer Research, 72, 527-536.

66. Eide, T., Ramberg, H., Glackin, C., Tindall, D., \& Tasken, K. A. (2013). TWIST1, A novel androgen-regulated gene, is a target for NKX3-1 in prostate cancer cells. Cancer Cell International, 13, 4.

67. Lu, T., Lin, W. J., Izumi, K., Wang, X., Xu, D., Fang, L. Y., Li, L., Jiang, Q., Jin, J., \& Chang, C. (2012). Targeting androgen receptor to suppress macrophage-induced EMT and benign prostatic hyperplasia (BPH) development. Molecular Endocrinology, 26, 17071715.

68. Pourmand, G., Ziaee, A. A., Abedi, A. R., Mehrsai, A., Alavi, H. A., Ahmadi, A., \& Saadati, H. R. (2007). Role of PTEN gene in progression of prostate cancer. Urology Journal, 4, 95-100. Spring.

69. Mulholland, D. J., Kobayashi, N., Ruscetti, M., Zhi, A., Tran, L. M., Huang, J., Gleave, M., \& Wu, H. (2012). Pten loss and RAS/MAPK activation cooperate to promote EMT and metastasis initiated from prostate cancer stem/progenitor cells. Cancer Research, 72, 18781889.

70. Dubrovska, A., Kim, S., Salamone, R. J., Walker, J. R., Maira, S. M., Garcia-Echeverria, C., Schultz, P. G., \& Reddy, V. A. (2009). The role of PTEN/Akt/PI3K signaling in the maintenance and viability of prostate cancer stem-like cell populations. Proceedings of the National Academy of Sciences of the United States of America, 106, 268-273.

71. Taylor, B. S., Schultz, N., Hieronymus, H., Gopalan, A., Xiao, Y., Carver, B. S., Arora, V. K., Kaushik, P., Cerami, E., Reva, B., Antipin, Y., Mitsiades, N., Landers, T., Dolgalev, I., Major, J. E., Wilson, M., Socci, N. D., Lash, A. E., Heguy, A., Eastham, J. A., Scher, H. I., Reuter, V. E., Scardino, P. T., Sander, C., Sawyers, C. L., \& Gerald, W. L. (2010). Integrative genomic profiling of human prostate cancer. Cancer Cell, 18, 11-22.

72. Nusse, R., \& Varmus, H. (2012). Three decades of Wnts: A personal perspective on how a scientific field developed. EMBO Journal, 31, 2670-2684.

73. Yee, D. S., Tang, Y., Li, X., Liu, Z., Guo, Y., Ghaffar, S., McQueen, P., Atreya, D., Xie, J., Simoneau, A. R., Hoang, B. H., \& Zi, X. (2010). The Wnt inhibitory factor 1 restoration in prostate cancer cells was associated with reduced tumor growth, decreased capacity of cell migration and invasion and a reversal of epithelial to mesenchymal transition. Molecular Cancer, 9, 162.

74. Clevers, H. (2006). Wnt/beta-catenin signaling in development and disease. Cell, 127, 469-480.

75. Truica, C. I., Byers, S., \& Gelmann, E. P. (2000). Beta-catenin affects androgen receptor transcriptional activity and ligand specificity. Cancer Research, 60, 4709-4713.

76. Whitaker, H. C., Girling, J., Warren, A. Y., Leung, H., Mills, I. G., \& Neal, D. E. (2008). Alterations in beta-catenin expression and localization in prostate cancer. Prostate, 68, 1196-1205.

77. Wan, X., Liu, J., Lu, J. F., Tzelepi, V., Yang, J., Starbuck, M. W., Diao, L., Wang, J., Efstathiou, E., Vazquez, E. S., Troncoso, P.,
Maity, S. N., \& Navone, N. M. (2012). Activation of beta-catenin signaling in androgen receptor-negative prostate cancer cells. Clinical Cancer Research, 18, 726-736.

78. Cao, J., Chiarelli, C., Richman, O., Zarrabi, K., Kozarekar, P., \& Zucker, S. (2008). Membrane type 1 matrix metalloproteinase induces epithelial-to-mesenchymal transition in prostate cancer. The Journal of Biological Chemistry, 283, 6232-6240.

79. Xie, D., Gore, C., Liu, J., Pong, R. C., Mason, R., Hao, G., Long, M., Kabbani, W., Yu, L., Zhang, H., Chen, H., Sun, X., Boothman, D. A., Min, W., \& Hsieh, J. T. (2010). "Role of DAB2IP in modulating epithelial-to-mesenchymal transition and prostate cancer metastasis,". Proceedings of the National Academy of Sciences of the United States of America, 107, 2485-2490.

80. Min, J., Zaslavsky, A., Fedele, G., McLaughlin, S. K., Reczek, E. E., De Raedt, T., Guney, I., Strochlic, D. E., Macconaill, L. E., Beroukhim, R., Bronson, R. T., Ryeom, S., Hahn, W. C., Loda, M., \& Cichowski, K. (2010). "An oncogene-tumor suppressor cascade drives metastatic prostate cancer by coordinately activating Ras and nuclear factor-kappaB. Nature Medicine, 16, 286-294.

81. K. Wu, J. Liu, S. F. Tseng, C. Gore, Z. Ning, N. Sharifi, L. Fazli, M. Gleave, P. Kapur, G. Xiao, X. Sun, O. K. Oz, W. Min, G. Alexandrakis, C. R. Yang, C. L. Hsieh, H. C. Wu, D. He, D. Xie, and J. T. Hsieh,"The role of DAB2IP in androgen receptor activation during prostate cancer progression,"Oncogene, Apr 222013.

82. Jain, G., Cronauer, M. V., Schrader, M., Moller, P., \& Marienfeld, R. B. (2012). NF-kappaB signaling in prostate cancer: a promising therapeutic target? World Journal of Urology, 30, 303-310.

83. McCall, P., Bennett, L., Ahmad, I., Mackenzie, L. M., Forbes, I. W., Leung, H. Y., Sansom, O. J., Orange, C., Seywright, M., Underwood, M. A., \& Edwards, J. (2012). NFkappaB signalling is upregulated in a subset of castrate-resistant prostate cancer patients and correlates with disease progression. British Journal of Cancer, 107, 1554-1563.

84. Min, C., Eddy, S. F., Sherr, D. H., \& Sonenshein, G. E. (2008). NFkappaB and epithelial to mesenchymal transition of cancer. Journal of Cellular Biochemistry, 104, 733-744.

85. Luo, J. L., Tan, W., Ricono, J. M., Korchynskyi, O., Zhang, M., Gonias, S. L., Cheresh, D. A., \& Karin, M. (2007). Nuclear cytokine-activated IKKalpha controls prostate cancer metastasis by repressing Maspin. Nature, 446, 690-694.

86. Odero-Marah, V. A., Wang, R., Chu, G., Zayzafoon, M., Xu, J., Shi, C., Marshall, F. F., Zhau, H. E., \& Chung, L. W. (2008). Receptor activator of NF-kappaB Ligand (RANKL) expression is associated with epithelial to mesenchymal transition in human prostate cancer cells. Cell Research, 18, 858-870.

87. Kuo, P. L., Shen, K. H., Hung, S. H., \& Hsu, Y. L. (2012). CXCL1/ GROalpha increases cell migration and invasion of prostate cancer by decreasing fibulin-1 expression through NF-kappaB/HDAC1 epigenetic regulation. Carcinogenesis, 33, 2477-2487.

88. Bolitho, C., Hahn, M. A., Baxter, R. C., \& Marsh, D. J. (2010). The chemokine CXCL1 induces proliferation in epithelial ovarian cancer cells by transactivation of the epidermal growth factor receptor. Endocrine-Related Cancer, 17, 929-940.

89. Shiota, M., Zardan, A., Takeuchi, A., Kumano, M., Beraldi, E., Naito, S., Zoubeidi, A., \& Gleave, M. E. (2012). Clusterin mediates TGF-beta-induced epithelial-mesenchymal transition and metastasis via Twist 1 in prostate cancer cells. Cancer Research, 72, 5261-5272.

90. Mak, P., Leav, I., Pursell, B., Bae, D., Yang, X., Taglienti, C. A., Gouvin, L. M., Sharma, V. M., \& Mercurio, A. M. (2010). ERbeta impedes prostate cancer EMT by destabilizing HIF-1alpha and inhibiting VEGF-mediated snail nuclear localization: implications for Gleason grading. Cancer Cell, 17, 319-332.

91. Poon, S., Easterbrook-Smith, S. B., Rybchyn, M. S., Carver, J. A., \& Wilson, M. R. (2000). Clusterin is an ATP-independent 
chaperone with very broad substrate specificity that stabilizes stressed proteins in a folding-competent state. Biochemistry, 39, 15953-15960.

92. Chi, K. N., Hotte, S. J., Yu, E. Y., Tu, D., Eigl, B. J., Tannock, I., Saad, F., North, S., Powers, J., Gleave, M. E., \& Eisenhauer, E. A. (2010). Randomized phase II study of docetaxel and prednisone with or without OGX-011 in patients with metastatic castrationresistant prostate cancer. Journal of Clinical Oncology, 28, $4247-4254$.

93. Zoubeidi, A., Chi, K., \& Gleave, M. (2010). Targeting the cytoprotective chaperone, clusterin, for treatment of advanced cancer. Clinical Cancer Research, 16, 1088-1093.

94. Turley, R. S., Finger, E. C., Hempel, N., How, T., Fields, T. A., \& Blobe, G. C. (2007). The type III transforming growth factor-beta receptor as a novel tumor suppressor gene in prostate cancer. Cancer Research, 67, 1090-1098.

95. Ikushima, H., \& Miyazono, K. (2010). TGFbeta signalling: a complex web in cancer progression. Nature Reviews Cancer, 10, 415-424.

96. Adorno, M., Cordenonsi, M., Montagner, M., Dupont, S., Wong, C., Hann, B., Solari, A., Bobisse, S., Rondina, M. B., Guzzardo, V., Parenti, A. R., Rosato, A., Bicciato, S., Balmain, A., \& Piccolo, S. (2009). A Mutant-p53/Smad complex opposes p63 to empower TGFbeta-induced metastasis. Cell, 137, 87-98.

97. Ding, Z., Wu, C. J., Chu, G. C., Xiao, Y., Ho, D., Zhang, J., Perry, S. R., Labrot, E. S., Wu, X., Lis, R., Hoshida, Y., Hiller, D., Hu, B., Jiang, S., Zheng, H., Stegh, A. H., Scott, K. L., Signoretti, S., Bardeesy, N., Wang, Y. A., Hill, D. E., Golub, T. R., Stampfer, M. J., Wong, W. H., Loda, M., Mucci, L., Chin, L., \& DePinho, R. A. (2011). SMAD4-dependent barrier constrains prostate cancer growth and metastatic progression. Nature, 470, 269-273.

98. Qin, J., Wu, S. P., Creighton, C. J., Dai, F., Xie, X., Cheng, C. M., Frolov, A., Ayala, G., Lin, X., Feng, X. H., Ittmann, M. M., Tsai, S. J., Tsai, M. J., \& Tsai, S. Y. (2013). COUP-TFII inhibits TGF-betainduced growth barrier to promote prostate tumorigenesis. Nature, 493, 236-240.

99. Buijs, J. T., Henriquez, N. V., van Overveld, P. G., van der Horst, G., ten Dijke, P., \& van der Pluijm, G. (2007). TGF-beta and BMP7 interactions in tumour progression and bone metastasis. Clinical \& Experimental Metastasis, 24, 609-617.

100. Grivennikov, S., Karin, E., Terzic, J., Mucida, D., Yu, G. Y., Vallabhapurapu, S., Scheller, J., Rose-John, S., Cheroutre, H., Eckmann, L., \& Karin, M. (2009). IL-6 and Stat3 are required for survival of intestinal epithelial cells and development of colitisassociated cancer. Cancer Cell, 15, 103-113.

101. Bollrath, J., Phesse, T. J., von Burstin, V. A., Putoczki, T., Bennecke, M., Bateman, T., Nebelsiek, T., Lundgren-May, T., Canli, O., Schwitalla, S., Matthews, V., Schmid, R. M., Kirchner, T., Arkan, M. C., Ernst, M., \& Greten, F. R. (2009). gp130mediated Stat 3 activation in enterocytes regulates cell survival and cell-cycle progression during colitis-associated tumorigenesis. Cancer Cell, 15, 91-102.

102. Yadav, A., Kumar, B., Datta, J., Teknos, T. N., \& Kumar, P. (2011). IL-6 promotes head and neck tumor metastasis by inducing epithelial-mesenchymal transition via the JAK-STAT3-SNAIL signaling pathway. Molecular Cancer Research, 9, 1658-1667.

103. Yao, Z., Fenoglio, S., Gao, D. C., Camiolo, M., Stiles, B., Lindsted, T., Schlederer, M., Johns, C., Altorki, N., Mittal, V., Kenner, L., \& Sordella, R. (2010). TGF-beta IL-6 axis mediates selective and adaptive mechanisms of resistance to molecular targeted therapy in lung cancer. Proceedings of the National Academy of Sciences of the United States of America, 107, 15535-15540.

104. Sullivan, N. J., Sasser, A. K., Axel, A. E., Vesuna, F., Raman, V., Ramirez, N., Oberyszyn, T. M., \& Hall, B. M. (2009). Interleukin-6 induces an epithelial-mesenchymal transition phenotype in human breast cancer cells. Oncogene, 28, 2940-2947.
105. Tenniswood, M. P., Guenette, R. S., Lakins, J., Mooibroek, M., Wong, P., \& Welsh, J. E. (1992). Active cell death in hormonedependent tissues. Cancer Metastasis Reviews, 11, 197-220.

106. Paul, C., Manero, F., Gonin, S., Kretz-Remy, C., Virot, S., \& Arrigo, A. P. (2002). Hsp27 as a negative regulator of cytochrome C release. Molecular and Cellular Biology, 22, 816-834.

107. Zoubeidi, A., \& Gleave, M. (2012). Small heat shock proteins in cancer therapy and prognosis. The International Journal of Biochemistry \& Cell Biology, 44, 1646-1656.

108. Shiota, M., Bishop, J. L., Nip, K. M., Zardan, A., Takeuchi, A., Cordonnier, T., Beraldi, E., Bazov, J., Fazli, L., Chi, K., Gleave, M., \& Zoubeidi, A. (2013). Hsp27 regulates epithelial mesenchymal transition, metastasis, and circulating tumor cells in prostate cancer. Cancer Research, 73, 3109-3119.

109. Lue, H. W., Yang, X., Wang, R., Qian, W., Xu, R. Z., Lyles, R., Osunkoya, A. O., Zhou, B. P., Vessella, R. L., Zayzafoon, M., Liu, Z. R., Zhau, H. E., \& Chung, L. W. (2011). LIV-1 promotes prostate cancer epithelial-to-mesenchymal transition and metastasis through HB-EGF shedding and EGFR-mediated ERK signaling. PLoS One, 6, e27720.

110. Mimeault, M., \& Batra, S. K. (2010). Divergent molecular mechanisms underlying the pleiotropic functions of macrophage inhibitory cytokine- 1 in cancer. Journal of Cellular Physiology, 224, 626-635.

111. Cheung, P. K., Woolcock, B., Adomat, H., Sutcliffe, M., Bainbridge, T. C., Jones, E. C., Webber, D., Kinahan, T., Sadar, M., Gleave, M. E., \& Vielkind, J. (2004). Protein profiling of microdissected prostate tissue links growth differentiation factor 15 to prostate carcinogenesis. Cancer Research, 64, 5929-5933.

112. Nakamura, T., Scorilas, A., Stephan, C., Yousef, G. M., Kristiansen, G., Jung, K., \& Diamandis, E. P. (2003). Quantitative analysis of macrophage inhibitory cytokine-1 (MIC-1) gene expression in human prostatic tissues. British Journal of Cancer, 88, 1101-1104.

113. Veveris-Lowe, T. L., Lawrence, M. G., Collard, R. L., Bui, L., Herington, A. C., Nicol, D. L., \& Clements, J. A. (2005). Kallikrein 4 (hK4) and prostate-specific antigen (PSA) are associated with the loss of E-cadherin and an epithelial-mesenchymal transition (EMT)-like effect in prostate cancer cells. EndocrineRelated Cancer, 12, 631-643.

114. Jang, M. J., Baek, S. H., \& Kim, J. H. (2011). UCH-L1 promotes cancer metastasis in prostate cancer cells through EMT induction. Cancer Letters, 302, 128-135.

115. Wesche, J., Haglund, K., \& Haugsten, E. M. (2011). Fibroblast growth factors and their receptors in cancer. The Biochemical Journal, 437, 199-213.

116. Feng, S., Wang, F., Matsubara, A., Kan, M., \& McKeehan, W. L. (1997). Fibroblast growth factor receptor 2 limits and receptor 1 accelerates tumorigenicity of prostate epithelial cells. Cancer Research, 57, 5369-5378.

117. Kwabi-Addo, B., Ropiquet, F., Giri, D., \& Ittmann, M. (2001). Alternative splicing of fibroblast growth factor receptors in human prostate cancer. Prostate, 46, 163-172.

118. Carstens, R. P., Wagner, E. J., \& Garcia-Blanco, M. A. (2000). An intronic splicing silencer causes skipping of the IIIb exon of fibroblast growth factor receptor 2 through involvement of polypyrimidine tract binding protein. Molecular and Cellular Biology, 20, 7388-7400.

119. Baraniak, A. P., Chen, J. R., \& Garcia-Blanco, M. A. (2006). Fox-2 mediates epithelial cell-specific fibroblast growth factor receptor 2 exon choice. Molecular and Cellular Biology, 26, $1209-1222$.

120. Warzecha, C. C., Sato, T. K., Nabet, B., Hogenesch, J. B., \& Carstens, R. P. (2009). ESRP1 and ESRP2 are epithelial cell-type-specific regulators of FGFR2 splicing. Molecular Cell, 33, 591-601.

121. Shapiro, I. M., Cheng, A. W., Flytzanis, N. C., Balsamo, M., Condeelis, J. S., Oktay, M. H., Burge, C. B., \& Gertler, F. B. 
(2011). An EMT-driven alternative splicing program occurs in human breast cancer and modulates cellular phenotype. PLoS Genetics, 7, e1002218.

122. Acevedo, V. D., Gangula, R. D., Freeman, K. W., Li, R., Zhang, Y., Wang, F., Ayala, G. E., Peterson, L. E., Ittmann, M., \& Spencer, D. M. (2007). Inducible FGFR-1 activation leads to irreversible prostate adenocarcinoma and an epithelial-to-mesenchymal transition. Cancer Cell, 12, 559-571.

123. Bao, B., Ahmad, A., Kong, D., Ali, S., Azmi, A. S., Li, Y., Banerjee, S., Padhye, S., \& Sarkar, F. H. (2012). Hypoxia induced aggressiveness of prostate cancer cells is linked with deregulated expression of VEGF, IL-6 and miRNAs that are attenuated by CDF. PLoS One, 7, e43726.

124. Wang, G. L., \& Semenza, G. L. (1993). General involvement of hypoxia-inducible factor 1 in transcriptional response to hypoxia. Proceedings of the National Academy of Sciences of the United States of America, 90, 4304-4308.

125. Zhao, J. H., Luo, Y., Jiang, Y. G., He, D. L., \& Wu, C. T. (2011). Knockdown of beta-catenin through shRNA cause a reversal of EMT and metastatic phenotypes induced by HIF-1alpha. Cancer Investigation, 29, 377-382.

126. Luo, Y., He, D. L., Ning, L., Shen, S. L., Li, L., \& Li, X. (2006). Hypoxia-inducible factor-1alpha induces the epithelial-mesenchymal transition of human prostatecancer cells. Chinese Medical Journal, 119, 713-718.

127. Yang, M. H., Wu, M. Z., Chiou, S. H., Chen, P. M., Chang, S. Y., Liu, C. J., Teng, S. C., \& Wu, K. J. (2008). Direct regulation of TWIST by HIF-1alpha promotes metastasis. Nature Cell Biology, 10, 295-305.

128. Sun, S., Ning, X., Zhang, Y., Lu, Y., Nie, Y., Han, S., Liu, L., Du, R., Xia, L., He, L., \& Fan, D. (2009). Hypoxia-inducible factor-1alpha induces Twist expression in tubular epithelial cells subjected to hypoxia, leading to epithelial-to-mesenchymal transition. Kidney International, 75, 1278-1287.

129. Song, X., Wang, H., Logsdon, C. D., Rashid, A., Fleming, J. B., Abbruzzese, J. L., Gomez, H. F., \& Evans, D. B. (2011). Overexpression of receptor tyrosine kinase Axl promotes tumor cell invasion and survival in pancreatic ductal adenocarcinoma. Cancer, 117, 734-743.

130. Mudduluru, G., Vajkoczy, P., \& Allgayer, H. (2010). Myeloid zinc finger 1 induces migration, invasion, and in vivo metastasis through Axl gene expression in solid cancer. Molecular Cancer Research, 8, 159-169.

131. Gustafsson, A., Bostrom, A. K., Ljungberg, B., Axelson, H., \& Dahlback, B. (2009). Gas6 and the receptor tyrosine kinase Axl in clear cell renal cell carcinoma. PLoS One, 4, e7575.

132. Gjerdrum, C., Tiron, C., Hoiby, T., Stefansson, I., Haugen, H., Sandal, T., Collett, K., Li, S., McCormack, E., Gjertsen, B. T., Micklem, D. R., Akslen, L. A., Glackin, C., \& Lorens, J. B. (2010). Axl is an essential epithelial-to-mesenchymal transitioninduced regulator of breast cancer metastasis and patient survival. Proceedings of the National Academy of Sciences of the United States of America, 107, 1124-1129.

133. Vajkoczy, P., Knyazev, P., Kunkel, A., Capelle, H. H., Behrndt, S., von Tengg-Kobligk, H., Kiessling, F., Eichelsbacher, U., Essig, M., Read, T. A., Erber, R., \& Ullrich, A. (2006). Dominant-negative inhibition of the Axl receptor tyrosine kinase suppresses brain tumor cell growth and invasion and prolongs survival. Proceedings of the National Academy of Sciences of the United States of America, 103, 5799-5804.

134. Hector, A., Montgomery, E. A., Karikari, C., Canto, M., Dunbar, K. B., Wang, J. S., Feldmann, G., Hong, S. M., Haffner, M. C., Meeker, A. K., Holland, S. J., Yu, J., Heckrodt, T. J., Zhang, J., Ding, P., Goff, D., Singh, R., Roa, J. C., Marimuthu, A., Riggins, G. J., Eshleman, J. R., Nelkin, B. D., Pandey, A., \& Maitra, A. (2010). The Axl receptor tyrosine kinase is an adverse prognostic factor and a therapeutic target in esophageal adenocarcinoma. Cancer Biology \& Therapy, 10, 1009-1018.

135. Koorstra, J. B., Karikari, C. A., Feldmann, G., Bisht, S., Rojas, P. L., Offerhaus, G. J., Alvarez, H., \& Maitra, A. (2009). The Axl receptor tyrosine kinase confers an adverse prognostic influence in pancreatic cancer and represents a new therapeutic target. Cancer Biology \& Therapy, 8, 618-626.

136. Gustafsson, A., Martuszewska, D., Johansson, M., Ekman, C., Hafizi, S., Ljungberg, B., \& Dahlback, B. (2009). Differential expression of Axl and Gas6 in renal cell carcinoma reflecting tumor advancement and survival. Clinical Cancer Research, 15, 4742-4749.

137. Zhang, Y. X., Knyazev, P. G., Cheburkin, Y. V., Sharma, K., Knyazev, Y. P., Orfi, L., Szabadkai, I., Daub, H., Keri, G., \& Ullrich, A. (2008). AXL is a potential target for therapeutic intervention in breast cancer progression. Cancer Research, 68, 1905-1915.

138. Hutterer, M., Knyazev, P., Abate, A., Reschke, M., Maier, H., Stefanova, N., Knyazeva, T., Barbieri, V., Reindl, M., Muigg, A., Kostron, H., Stockhammer, G., \& Ullrich, A. (2008). Axl and growth arrest-specific gene 6 are frequently overexpressed in human gliomas and predict poor prognosis in patients with glioblastoma multiforme. Clinical Cancer Research, 14, 130-138.

139. Mishra, A., Wang, J., Shiozawa, Y., McGee, S., Kim, J., Jung, Y., Joseph, J., Berry, J. E., Havens, A., Pienta, K. J., \& Taichman, R. S. (2012). Hypoxia stabilizes GAS6/Axl signaling in metastatic prostate cancer. Molecular Cancer Research, 10, 703-712.

140. Kalluri, R., \& Zeisberg, M. (2006). Fibroblasts in cancer. Nature Reviews Cancer, 6, 392-401.

141. Liotta, L. A., \& Kohn, E. C. (2001). The microenvironment of the tumour-host interface. Nature, 411, 375-379.

142. Chung, L. W., Baseman, A., Assikis, V., \& Zhau, H. E. (2005). Molecular insights into prostate cancer progression: The missing link of tumor microenvironment. The Journal of Urology, 173, 10-20.

143. Kaminski, A., Hahne, J. C., Haddouti el, M., Florin, A., Wellmann, A., \& Wernert, N. (2006). Tumour-stroma interactions between metastatic prostate cancer cells and fibroblasts. International Journal of Molecular Medicine, 18, 941-950.

144. Giannoni, E., Bianchini, F., Calorini, L., \& Chiarugi, P. (2011). Cancer associated fibroblasts exploit reactive oxygen species through a proinflammatory signature leading to epithelial mesenchymal transition and stemness. Antioxidants \& Redox Signaling, 14, 2361-2371.

145. Lafon, C., Mathieu, C., Guerrin, M., Pierre, O., Vidal, S., \& Valette, A. (1996). Transforming growth factor beta 1 -induced apoptosis in human ovarian carcinoma cells: protection by the antioxidant Nacetylcysteine and bcl-2. Cell Growth \& Differentiation, 7, 10951104.

146. Rhyu, D. Y., Yang, Y., Ha, H., Lee, G. T., Song, J. S., Uh, S. T., \& Lee, H. B. (2005). Role of reactive oxygen species in TGF-beta1induced mitogen-activated protein kinase activation and epithelialmesenchymal transition in renal tubular epithelial cells. Journal of the American Society of Nephrology, 16, 667-675.

147. Radisky, D. C., Levy, D. D., Littlepage, L. E., Liu, H., Nelson, C. M., Fata, J. E., Leake, D., Godden, E. L., Albertson, D. G., Nieto, M. A., Werb, Z., \& Bissell, M. J. (2005). Raclb and reactive oxygen species mediate MMP-3-induced EMT and genomic instability. Nature, 436, 123-127.

148. Barnett, P., Arnold, R. S., Mezencev, R., Chung, L. W., Zayzafoon, M., \& Odero-Marah, V. (2011). Snail-mediated regulation of reactive oxygen species in ARCaP human prostate cancer cells. Biochemical and Biophysical Research Communications, 404, 34-39.

149. Das, T. P., Suman, S., \& Damodaran, C. (2013). Reactive oxygen species generation inhibits epithelial-mesenchymal transition and promotes growth arrest in prostate cancer cells. Molecular Carcinogenesis. doi:10.1002/mc.22014.

150. Sun, Y., Campisi, J., Higano, C., Beer, T. M., Porter, P., Coleman, I., True, L., \& Nelson, P. S. (2012). Treatment-induced damage to the 
tumor microenvironment promotes prostate cancer therapy resistance through WNT16B. Nature Medicine, 18, 1359-1368.

151. Zhang, Y., Daquinag, A., Traktuev, D. O., Amaya-Manzanares, F., Simmons, P. J., March, K. L., Pasqualini, R., Arap, W., \& Kolonin, M. G. (2009). White adipose tissue cells are recruited by experimental tumors and promote cancer progression in mouse models. Cancer Research, 69, 5259-5266.

152. Chao, Y., Wu, Q., Shepard, C., \& Wells, A. (2012). Hepatocyte induced re-expression of E-cadherin in breast and prostate cancer cells increases chemoresistance. Clinical \& Experimental Metastasis, 29, 39-50.

153. Yates, C. C., Shepard, C. R., Stolz, D. B., \& Wells, A. (2007). Coculturing human prostate carcinoma cells with hepatocytes leads to increased expression of E-cadherin. British Journal of Cancer, 96, $1246-1252$

154. S. Josson, S. Sharp, S. Y. Sung, P. A. Johnstone, R. Aneja, R. Wang, M. Gururajan, T. Turner, L. W. Chung, and C. Yates (2010) Tumorstromal interactions influence radiation sensitivity in epithelialversus mesenchymal-like prostate cancer cells. Journal of Oncology, 2010. doi:10.1155/2010/232831.

155. Oltean, S., Sorg, B. S., Albrecht, T., Bonano, V. I., Brazas, R. M., Dewhirst, M. W., \& Garcia-Blanco, M. A. (2006). Alternative inclusion of fibroblast growth factor receptor 2 exon IIIc in Dunning prostate tumors reveals unexpected epithelial mesenchymal plasticity. Proceedings of the National Academy of Sciences of the United States of America, 103, 14116-14121.

156. Giannini, G., Cabri, W., Fattorusso, C., \& Rodriquez, M. (2012). Histone deacetylase inhibitors in the treatment of cancer: Overview and perspectives. Future Medicinal Chemistry, 4, 1439-1460.

157. Kong, D., Ahmad, A., Bao, B., Li, Y., Banerjee, S., \& Sarkar, F. H. (2012). Histone deacetylase inhibitors induce epithelial-tomesenchymal transition in prostate cancer cells. PLoS One, 7, e45045.

158. Bradley, D., Rathkopf, D., Dunn, R., Stadler, W. M., Liu, G., Smith, D. C., Pili, R., Zwiebel, J., Scher, H., \& Hussain, M. (2009). Vorinostat in advanced prostate cancer patients progressing on prior chemotherapy (National Cancer Institute Trial 6862): trial results and interleukin-6 analysis: A study by the Department of Defense Prostate Cancer Clinical Trial Consortium and University of Chicago Phase 2 Consortium. Cancer, 115, 5541-5549.

159. Martinez-Garcia, E., Popovic, R., Min, D. J., Sweet, S. M., Thomas, P. M., Zamdborg, L., Heffner, A., Will, C., Lamy, L., Staudt, L. M., Levens, D. L., Kelleher, N. L., \& Licht, J. D. (2011). The MMSET histone methyl transferase switches global histone methylation and alters gene expression in $\mathrm{t}(4 ; 14)$ multiple myeloma cells. Blood, 117 , 211-220.

160. Hudlebusch, H. R., Skotte, J., Santoni-Rugiu, E., Zimling, Z. G., Lees, M. J., Simon, R., Sauter, G., Rota, R., De Ioris, M. A., Quarto, M., Johansen, J. V., Jorgensen, M., Rechnitzer, C., Maroun, L. L., Schroder, H., Petersen, B. L., \& Helin, K. (2011). MMSET is highly expressed and associated with aggressiveness in neuroblastoma. Cancer Research, 71, 4226-4235.

161. Kassambara, A., Klein, B., \& Moreaux, J. (2009). MMSET is overexpressed in cancers: link with tumor aggressiveness. Biochemical and Biophysical Research Communications, 379, 840-845.

162. Ezponda, T., Popovic, R., Shah, M. Y., Martinez-Garcia, E., Zheng, Y., Min, D. J., Will, C., Neri, A., Kelleher, N. L., Yu, J., \& Licht, J. D. (2013). The histone methyltransferase MMSET/WHSC1 activates TWIST1 to promote an epithelial-mesenchymal transition and invasive properties of prostate cancer. Oncogene, 32, 2882-2890.

163. Smith, J. (2002). Human Sir2 and the "silencing" of p53 activity. Trends in Cell Biology, 12, 404-406.

164. Giannakou, M. E., \& Partridge, L. (2004). The interaction between FOXO and SIRT1: Tipping the balance towards survival. Trends in Cell Biology, 14, 408-412.
165. Byles, V., Zhu, L., Lovaas, J. D., Chmilewski, L. K., Wang, J., Faller, D. V., \& Dai, Y. (2012). SIRT1 induces EMT by cooperating with EMT transcription factors and enhances prostate cancer cell migration and metastasis. Oncogene, 31, 4619-4629.

166. Cao, Q., Yu, J., Dhanasekaran, S. M., Kim, J. H., Mani, R. S., Tomlins, S. A., Mehra, R., Laxman, B., Cao, X., Kleer, C. G., Varambally, S., \& Chinnaiyan, A. M. (2008). Repression of Ecadherin by the polycomb group protein EZH2 in cancer. Oncogene, 27, 7274-7284.

167. Saha, B., Kaur, P., Tsao-Wei, D., Naritoku, W. Y., Groshen, S., Datar, R. H., Jones, L. W., \& Imam, S. A. (2008). Unmethylated E-cadherin gene expression is significantly associated with metastatic human prostate cancer cells in bone. Prostate, 68, 1681-1688.

168. Jansson, M. D., \& Lund, A. H. (2012). MicroRNA and cancer. Molecular Oncology, 6, 590-610.

169. Bartel, D. P. (2004). MicroRNAs: Genomics, biogenesis, mechanism, and function. Cell, 116, 281-297.

170. Bullock, M. D., Sayan, A. E., Packham, G. K., \& Mirnezami, A. H. (2012). MicroRNAs: critical regulators of epithelial to mesenchymal (EMT) and mesenchymal to epithelial transition (MET) in cancer progression. Biology of the Cell, 104, 3-12.

171. Liu, Y. N., Yin, J. J., Abou-Kheir, W., Hynes, P. G., Casey, O. M., Fang, L., Yi, M., Stephens, R. M., Seng, V., Sheppard-Tillman, H., Martin, P., \& Kelly, K. (2013). MiR-1 and miR-200 inhibit EMT via Slug-dependent and tumorigenesis via Slug-independent mechanisms. Oncogene, 32, 296-306.

172. Kong, D., Li, Y., Wang, Z., Banerjee, S., Ahmad, A., Kim, H. R., \& Sarkar, F. H. (2009). miR-200 regulates PDGF-D-mediated epithelial-mesenchymal transition, adhesion, and invasion of prostate cancer cells. Stem Cells, 27, 1712-1721.

173. Kong, D., Banerjee, S., Ahmad, A., Li, Y., Wang, Z., Sethi, S., \& Sarkar, F. H. (2010). Epithelial to mesenchymal transition is mechanistically linked with stem cell signatures in prostate cancer cells. PLoS One, 5, e12445.

174. Puhr, M., Hoefer, J., Schafer, G., Erb, H. H., Oh, S. J., Klocker, H., Heidegger, I., Neuwirt, H., \& Culig, Z. (2012). Epithelial-tomesenchymal transition leads to docetaxel resistance in prostate cancer and is mediated by reduced expression of miR-200c and miR-205. The American Journal of Pathology, 181, 2188-2201.

175. Qu, Y., Li, W. C., Hellem, M. R., Rostad, K., Popa, M., McCormack, E., Oyan, A. M., Kalland, K. H., \& Ke, X. S. (2013). MiR-182 and miR-203 induce mesenchymal to epithelial transition and self-sufficiency of growth signals via repressing SNAI2 in prostate cells. International Journal of Cancer, 133, 544-555.

176. Coppola, V., Musumeci, M., Patrizii, M., Cannistraci, A., Addario, A., Maugeri-Sacca, M., Biffoni, M., Francescangeli, F., Cordenonsi, M., Piccolo, S., Memeo, L., Pagliuca, A., Muto, G., Zeuner, A., De Maria, R., \& Bonci, D. (2013). BTG2 loss and miR-21 upregulation contribute to prostate cell transformation by inducing luminal markers expression and epithelial-mesenchymal transition. Oncogene, 32, 1843-1853.

177. Watahiki, A., Macfarlane, R. J., Gleave, M. E., Crea, F., Wang, Y., Helgason, C. D., \& Chi, K. N. (2013). Plasma miRNAs as biomarkers to identify patients with castration-resistant metastatic prostate cancer. International Journal of Molecular Sciences, 14, 77577770 .

178. Ru, P., Steele, R., Newhall, P., Phillips, N. J., Toth, K., \& Ray, R. B. (2012). miRNA-29b suppresses prostate cancer metastasis by regulating epithelial-mesenchymal transition signaling. Molecular Cancer Therapeutics, 11, 1166-1173.

179. Tucci, P., Agostini, M., Grespi, F., Markert, E. K., Terrinoni, A., Vousden, K. H., Muller, P. A., Dotsch, V., Kehrloesser, S., Sayan, B. S., Giaccone, G., Lowe, S. W., Takahashi, N., Vandenabeele, P., Knight, R. A., Levine, A. J., \& Melino, G. (2012). Loss of p63 and its microRNA-205 target results in enhanced cell migration and 
metastasis in prostate cancer. Proceedings of the National Academy of Sciences of the United States of America, 109, 15312-15317.

180. Zi, X., \& Agarwal, R. (1999). Silibinin decreases prostate-specific antigen with cell growth inhibition via G1 arrest, leading to differentiation of prostate carcinoma cells: Implications for prostate cancer intervention. Proceedings of the National Academy of Sciences of the United States of America, 96, 7490-7495.

181. Tyagi, A. K., Singh, R. P., Agarwal, C., Chan, D. C., \& Agarwal, R. (2002). Silibinin strongly synergizes human prostate carcinoma DU145 cells to doxorubicin-induced growth Inhibition, G2-M arrest, and apoptosis. Clinical Cancer Research, 8, 3512-3519.

182. Dhanalakshmi, S., Agarwal, P., Glode, L. M., \& Agarwal, R. (2003). Silibinin sensitizes human prostate carcinoma DU145 cells to cisplatin- and carboplatin-induced growth inhibition and apoptotic death. International Journal of Cancer, 106, 699-705.

183. Flaig, T. W., Su, L. J., Harrison, G., Agarwal, R., \& Glode, L. M. (2007). Silibinin synergizes with mitoxantrone to inhibit cell growth and induce apoptosis in human prostate cancer cells. International Journal of Cancer, 120, 2028-2033.

184. Tyagi, A., Bhatia, N., Condon, M. S., Bosland, M. C., Agarwal, C., \& Agarwal, R. (2002). Antiproliferative and apoptotic effects of silibinin in rat prostate cancer cells. Prostate, 53, 211-217.

185. Agarwal, C., Tyagi, A., Kaur, M., \& Agarwal, R. (2007). Silibinin inhibits constitutive activation of Stat3, and causes caspase activation and apoptotic death of human prostate carcinoma DU145 cells. Carcinogenesis, 28, 1463-1470.

186. Zhu, W., Zhang, J. S., \& Young, C. Y. (2001). Silymarin inhibits function of the androgen receptor by reducing nuclear localization of the receptor in the human prostate cancer cell line $\mathrm{LNCaP}$. Carcinogenesis, 22, 1399-1403.

187. Mokhtari, M. J., Motamed, N., \& Shokrgozar, M. A. (2008). Evaluation of silibinin on the viability, migration and adhesion of the human prostate adenocarcinoma (PC-3) cell line. Cell Biology International, 32, 888-892.

188. Wu, K. J., Zeng, J., Zhu, G. D., Zhang, L. L., Zhang, D., Li, L., Fan, J. H., Wang, X. Y., \& He, D. L. (2009). Silibinin inhibits prostate cancer invasion, motility and migration by suppressing vimentin and MMP-2 expression. Acta Pharmacologica Sinica, 30, 1162 1168 .

189. Wu, K., Zeng, J., Li, L., Fan, J., Zhang, D., Xue, Y., Zhu, G., Yang, L., Wang, X., \& He, D. (2010). Silibinin reverses epithelial-tomesenchymal transition in metastatic prostate cancer cells by targeting transcription factors. Oncology Reports, 23, 1545-1552.

190. Jung, H. J., Park, J. W., Lee, J. S., Lee, S. R., Jang, B. C., Suh, S. I., Suh, M. H., \& Baek, W. K. (2009). Silibinin inhibits expression of HIF-1alpha through suppression of protein translation in prostate cancer cells. Biochemical and Biophysical Research Communications, 390, 71-76.

191. Zhang, L. L., Li, L., Wu, D. P., Fan, J. H., Li, X., Wu, K. J., Wang, X. Y., \& He, D. L. (2008). A novel anti-cancer effect of genistein: reversal of epithelial mesenchymal transition in prostate cancer cells. Acta Pharmacologica Sinica, 29, 1060-1068.

192. Zhang, L., Li, L., Jiao, M., Wu, D., Wu, K., Li, X., Zhu, G., Yang, L., Wang, X., Hsieh, J. T., \& He, D. (2012). Genistein inhibits the stemness properties of prostate cancer cells through targeting Hedgehog-Gli1 pathway. Cancer Letters, 323, 48-57.

193. Chiyomaru, T., Yamamura, S., Fukuhara, S., Hidaka, H., Majid, S., Saini, S., Arora, S., Deng, G., Shahryari, V., Chang, I., Tanaka, Y., Tabatabai, Z. L., Enokida, H., Seki, N., Nakagawa, M., \& Dahiya, R. (2013). Genistein up-regulates tumor suppressor microRNA574-3p in prostate cancer. PLoS One, 8, e58929.

194. Baritaki, S., Chapman, A., Yeung, K., Spandidos, D. A., Palladino, M., \& Bonavida, B. (2009). Inhibition of epithelial to mesenchymal transition in metastatic prostate cancer cells by the novel proteasome inhibitor, NPI-0052: pivotal roles of Snail repression and RKIP induction. Oncogene, 28, 3573-3585.
195. Morel, A. P., Lievre, M., Thomas, C., Hinkal, G., Ansieau, S., \& Puisieux, A. (2008). Generation of breast cancer stem cells through epithelial-mesenchymal transition. PLoS One, 3, e2888.

196. Lan, L., Luo, Y., Cui, D., Shi, B. Y., Deng, W., Huo, L. L., Chen, H. L., Zhang, G. Y., \& Deng, L. L. (2013). Epithelial-mesenchymal transition triggers cancer stem cell generation in human thyroid cancer cells. International Journal of Oncology, 43, 113-120.

197. Klarmann, G. J., Hurt, E. M., Mathews, L. A., Zhang, X., Duhagon, M. A., Mistree, T., Thomas, S. B., \& Farrar, W. L. (2009). Invasive prostate cancer cells are tumor initiating cells that have a stem celllike genomic signature. Clinical \& Experimental Metastasis, 26, 433-446.

198. Albino, D., Longoni, N., Curti, L., Mello-Grand, M., Pinton, S., Civenni, G., Thalmann, G., D'Ambrosio, G., Sarti, M., Sessa, F., Chiorino, G., Catapano, C. V., \& Carbone, G. M. (2012). ESE3/EHF controls epithelial cell differentiation and its loss leads to prostate tumors with mesenchymal and stem-like features. Cancer Research, 72, 2889-2900.

199. Lukacs, R. U., Memarzadeh, S., Wu, H., \& Witte, O. N. (2010). Bmi-1 is a crucial regulator of prostate stem cell self-renewal and malignant transformation. Cell Stem Cell, 7, 682-693.

200. Domingo-Domenech, J., Vidal, S. J., Rodriguez-Bravo, V., CastilloMartin, M., Quinn, S. A., Rodriguez-Barrueco, R., Bonal, D. M., Charytonowicz, E., Gladoun, N., de la Iglesia-Vicente, J., Petrylak, D. P., Benson, M. C., Silva, J. M., \& Cordon-Cardo, C. (2012). Suppression of acquired docetaxel resistance in prostate cancer through depletion of Notch- and Hedgehog-dependent tumorinitiating cells. Cancer Cell, 22, 373-388.

201. Bae, K. M., Su, Z., Frye, C., McClellan, S., Allan, R. W., Andrejewski, J. T., Kelley, V., Jorgensen, M., Steindler, D. A., Vieweg, J., \& Siemann, D. W. (2010). Expression of pluripotent stem cell reprogramming factors by prostate tumor initiating cells. The Journal of Urology, 183, 2045-2053.

202. Yan, H., Chen, X., Zhang, Q., Qin, J., Li, H., Liu, C., CalhounDavis, T., Coletta, L. D., Klostergaard, J., Fokt, I., Skora, S., Priebe, W., Bi, Y., \& Tang, D. G. (2011). Drug-tolerant cancer cells show reduced tumor-initiating capacity: Depletion of CD44 cells and evidence for epigenetic mechanisms. PLoS One, 6, e24397.

203. Wang, Z. A., \& Shen, M. M. (2011). Revisiting the concept of cancer stem cells in prostate cancer. Oncogene, 30, 1261-1271.

204. Gupta, P. B., Onder, T. T., Jiang, G., Tao, K., Kuperwasser, C., Weinberg, R. A., \& Lander, E. S. (2009). Identification of selective inhibitors of cancer stem cells by high-throughput screening. Cell, 138, 645-659.

205. Allard, W. J., Matera, J., Miller, M. C., Repollet, M., Connelly, M. C., Rao, C., Tibbe, A. G., Uhr, J. W., \& Terstappen, L. W. (2004). Tumor cells circulate in the peripheral blood of all major carcinomas but not in healthy subjects or patients with nonmalignant diseases. Clinical Cancer Research, 10, 6897-6904.

206. Cristofanilli, M., Budd, G. T., Ellis, M. J., Stopeck, A., Matera, J., Miller, M. C., Reuben, J. M., Doyle, G. V., Allard, W. J., Terstappen, L. W., \& Hayes, D. F. (2004). Circulating tumor cells, disease progression, and survival in metastatic breast cancer. The New England Journal of Medicine, 351, 781-791.

207. de Bono, J. S., Scher, H. I., Montgomery, R. B., Parker, C., Miller, M. C., Tissing, H., Doyle, G. V., Terstappen, L. W., Pienta, K. J., \& Raghavan, D. (2008). Circulating tumor cells predict survival benefit from treatment in metastatic castration-resistant prostate cancer. Clinical Cancer Research, 14, 6302-6309.

208. Cohen, S. J., Punt, C. J., Iannotti, N., Saidman, B. H., Sabbath, K. D., Gabrail, N. Y., Picus, J., Morse, M., Mitchell, E., Miller, M. C., Doyle, G. V., Tissing, H., Terstappen, L. W., \& Meropol, N. J. (2008). Relationship of circulating tumor cells to tumor response, progression-free survival, and overall survival in patients with metastatic colorectal cancer. Journal of Clinical Oncology, 26, 3213-3221. 
209. Khan, M. S., Kirkwood, A., Tsigani, T., Garcia-Hernandez, J., Hartley, J. A., Caplin, M. E., \& Meyer, T. (2013). Circulating tumor cells as prognostic markers in neuroendocrine tumors. Journal of Clinical Oncology, 31, 365-372.

210. Scher, H. I., Morris, M. J., Basch, E., \& Heller, G. (2011). End points and outcomes in castration-resistant prostate cancer: From clinical trials to clinical practice. Journal of Clinical Oncology, 29, 3695-3704.

211. Lecharpentier, A., Vielh, P., Perez-Moreno, P., Planchard, D., Soria, J. C., \& Farace, F. (2011). Detection of circulating tumour cells with a hybrid (epithelial/mesenchymal) phenotype in patients with metastatic non-small cell lung cancer. British Journal of Cancer, 105, $1338-1341$.

212. Krebs, M. G., Hou, J. M., Sloane, R., Lancashire, L., Priest, L., Nonaka, D., Ward, T. H., Backen, A., Clack, G., Hughes, A., Ranson, M., Blackhall, F. H., \& Dive, C. (2012). Analysis of circulating tumor cells in patients with non-small cell lung cancer using epithelial marker-dependent and -independent approaches. Journal of Thoracic Oncology, 7, 306-315.

213. Bellizzi, A., Sebastian, S., Ceglia, P., Centonze, M., Divella, R., Manzillo, E. F., Azzariti, A., Silvestris, N., Montemurro, S., Caliandro, C., De Luca, R., Cicero, G., Rizzo, S., Russo, A., Quaranta, M., Simone, G., \& Paradiso, A. (2013). Co-expression of CD133(+)/CD44(+) in human colon cancer and liver metastasis. Journal of Cellular Physiology, 228, 408-415.

214. Barriere, G., Riouallon, A., Renaudie, J., Tartary, M., \& Rigaud, M. (2012). Mesenchymal and stemness circulating tumor cells in early breast cancer diagnosis. BMC Cancer, 12, 114.

215. Sieuwerts, A. M., Kraan, J., Bolt, J., van der Spoel, P., Elstrodt, F., Schutte, M., Martens, J. W., Gratama, J. W., Sleijfer, S., \& Foekens, J. A. (2009). Anti-epithelial cell adhesion molecule antibodies and the detection of circulating normal-like breast tumor cells. Journal of the National Cancer Institute, 101, 61-66.

216. Raimondi, C., Gradilone, A., Naso, G., Vincenzi, B., Petracca, A., Nicolazzo, C., Palazzo, A., Saltarelli, R., Spremberg, F., Cortesi, E., \& Gazzaniga, P. (2011). Epithelial-mesenchymal transition and stemness features in circulating tumor cells from breast cancer patients. Breast Cancer Research and Treatment, 130, 449-455.

217. Ozkumur, E., Shah, A. M., Ciciliano, J. C., Emmink, B. L., Miyamoto, D. T., Brachtel, E., Yu, M., Chen, P. I., Morgan, B., Trautwein, J., Kimura, A., Sengupta, S., Stott, S. L., Karabacak, N. M., Barber, T. A., Walsh, J. R., Smith, K., Spuhler, P. S., Sullivan, J. P., Lee, R. J., Ting, D. T., Luo, X., Shaw, A. T., Bardia, A., Sequist, L. V., Louis, D. N., Maheswaran, S., Kapur, R., Haber, D. A., \& Toner, M. (2013). "Inertial focusing for tumor antigen-dependent and -independent sorting of rare circulating tumor cells,". Science Translational Medicine, 5, 179ra47.

218. Ryan, C. J., Shah, S., Efstathiou, E., Smith, M. R., Taplin, M. E., Bubley, G. J., Logothetis, C. J., Kheoh, T., Kilian, C., Haqq, C. M., Molina, A., \& Small, E. J. (2011). Phase II study of abiraterone acetate in chemotherapy-naive metastatic castration-resistant prostate cancer displaying bone flare discordant with serologic response. Clinical Cancer Research, 17, 4854-4861.

219. Smith, D. C., Smith, M. R., Sweeney, C., Elfiky, A. A., Logothetis, C., Corn, P. G., Vogelzang, N. J., Small, E. J., Harzstark, A. L., Gordon, M. S., Vaishampayan, U. N., Haas, N. B., Spira, A. I., Lara, P. N., Jr., Lin, C. C., Srinivas, S., Sella, A., Schoffski, P., Scheffold, C., Weitzman, A. L., \& Hussain, M. (2013). Cabozantinib in patients with advanced prostate cancer: Results of a phase II randomized discontinuation trial. Journal of Clinical Oncology, 31, 412-419.

220. R. J. Lee, P. J. Saylor, M. D. Michaelson, S. M. Rothenberg, M. E. Smas, D. T. Miyamoto, C. A. Gurski, W. Xie, S. Maheswaran, D. A. Haber, J. G. Goldin, and M. R. Smith, "A Dose-Ranging Study of Cabozantinib in Men with Castration-Resistant Prostate Cancer and Bone Metastases," Clin Cancer Res, May 152013
221. Kwok, W. K., Ling, M. T., Lee, T. W., Lau, T. C., Zhou, C., Zhang, X., Chua, C. W., Chan, K. W., Chan, F. L., Glackin, C., Wong, Y. C., \& Wang, X. (2005). Up-regulation of TWIST in prostate cancer and its implication as a therapeutic target. Cancer Research, 65, 5153-5162.

222. Sethi, S., Macoska, J., Chen, W., \& Sarkar, F. H. (2010). Molecular signature of epithelial-mesenchymal transition (EMT) in human prostate cancer bone metastasis. American Journal of Translational Research, 3, 90-99.

223. Gravdal, K., Halvorsen, O. J., Haukaas, S. A., \& Akslen, L. A. (2007). A switch from E-cadherin to N-cadherin expression indicates epithelial to mesenchymal transition and is of strong and independent importance for the progress of prostate cancer. Clinical Cancer Research, 13, 7003-7011.

224. Behnsawy, H. M., Miyake, H., Harada, K., \& Fujisawa, M. (2013). Expression patterns of epithelial-mesenchymal transition markers in localized prostate cancer: Significance in clinicopathological outcomes following radical prostatectomy. BJU International, 111, $30-37$.

225. Tomlins, S. A., Rhodes, D. R., Perner, S., Dhanasekaran, S. M., Mehra, R., Sun, X. W., Varambally, S., Cao, X., Tchinda, J., Kuefer, R., Lee, C., Montie, J. E., Shah, R. B., Pienta, K. J., Rubin, M. A., \& Chinnaiyan, A. M. (2005). Recurrent fusion of TMPRSS2 and ETS transcription factor genes in prostate cancer. Science, 310, 644-648.

226. Tomlins, S. A., Laxman, B., Dhanasekaran, S. M., Helgeson, B. E., Cao, X., Morris, D. S., Menon, A., Jing, X., Cao, Q., Han, B., Yu, J., Wang, L., Montie, J. E., Rubin, M. A., Pienta, K. J., Roulston, D., Shah, R. B., Varambally, S., Mehra, R., \& Chinnaiyan, A. M. (2007). Distinct classes of chromosomal rearrangements create oncogenic ETS gene fusions in prostate cancer. Nature, 448, 595-599.

227. Gupta, S., Iljin, K., Sara, H., Mpindi, J. P., Mirtti, T., Vainio, P., Rantala, J., Alanen, K., Nees, M., \& Kallioniemi, O. (2010). FZD4 as a mediator of ERG oncogene-induced WNT signaling and epithelial-to-mesenchymal transition in human prostate cancer cells. Cancer Research, 70, 6735-6745.

228. Leshem, O., Madar, S., Kogan-Sakin, I., Kamer, I., Goldstein, I., Brosh, R., Cohen, Y., Jacob-Hirsch, J., Ehrlich, M., Ben-Sasson, S., Goldfinger, N., Loewenthal, R., Gazit, E., Rotter, V., \& Berger, R. (2011). TMPRSS2/ERG promotes epithelial to mesenchymal transition through the ZEB1/ZEB2 axis in a prostate cancer model. PLoS One, 6, e21650.

229. Demichelis, F., Fall, K., Perner, S., Andren, O., Schmidt, F., Setlur, S. R., Hoshida, Y., Mosquera, J. M., Pawitan, Y., Lee, C., Adami, H. O., Mucci, L. A., Kantoff, P. W., Andersson, S. O., Chinnaiyan, A. M., Johansson, J. E., \& Rubin, M. A. (2007). TMPRSS2:ERG gene fusion associated with lethal prostate cancer in a watchful waiting cohort. Oncogene, 26, 4596-4599.

230. Nam, R. K., Sugar, L., Yang, W., Srivastava, S., Klotz, L. H., Yang, L. Y., Stanimirovic, A., Encioiu, E., Neill, M., Loblaw, D. A., Trachtenberg, J., Narod, S. A., \& Seth, A. (2007). Expression of the TMPRSS2:ERG fusion gene predicts cancer recurrence after surgery for localised prostate cancer. British Journal of Cancer, 97, 1690-1695.

231. Attard, G., Clark, J., Ambroisine, L., Fisher, G., Kovacs, G., Flohr, P., Berney, D., Foster, C. S., Fletcher, A., Gerald, W. L., Moller, H., Reuter, V., De Bono, J. S., Scardino, P., Cuzick, J., \& Cooper, C. S. (2008). Duplication of the fusion of TMPRSS2 to ERG sequences identifies fatal human prostate cancer. Oncogene, 27, 253-263.

232. Pettersson, A., Graff, R. E., Bauer, S. R., Pitt, M. J., Lis, R. T., Stack, E. C., Martin, N. E., Kunz, L., Penney, K. L., Ligon, A. H., Suppan, C., Flavin, R., Sesso, H. D., Rider, J. R., Sweeney, C., Stampfer, M. J., Fiorentino, M., Kantoff, P. W., Sanda, M. G., Giovannucci, E. L., Ding, E. L., Loda, M., \& Mucci, L. A. (2012). The TMPRSS2:ERG rearrangement, ERG expression, and prostate cancer outcomes: A cohort study and meta-analysis. Cancer Epidemiology, Biomarkers \& Prevention, 21, 1497-1509. 
233. Chen, C. L., Mahalingam, D., Osmulski, P., Jadhav, R. R., Wang, C. M., Leach, R. J., Chang, T. C., Weitman, S. D., Kumar, A. P., Sun, L., Gaczynska, M. E., Thompson, I. M., \& Huang, T. H. (2013). Single-cell analysis of circulating tumor cells identifies cumulative expression patterns of EMT-related genes in metastatic prostate cancer. Prostate, 73, 813-826.

234. Jennbacken, K., Tesan, T., Wang, W., Gustavsson, H., Damber, J. E., \& Welen, K. (2010). N-Cadherin increases after androgen deprivation and is associated with metastasis in prostate cancer. Endocrine-Related Cancer, 17, 469-479.

235. Tanaka, H., Kono, E., Tran, C. P., Miyazaki, H., Yamashiro, J., Shimomura, T., Fazli, L., Wada, R., Huang, J., Vessella, R. L., An, J., Horvath, S., Gleave, M., Rettig, M. B., Wainberg, Z. A., \& Reiter, R. E. (2010). Monoclonal antibody targeting of N-cadherin inhibits prostate cancer growth, metastasis and castration resistance. Nature Medicine, 16, 1414-1420.

236. Putzke, A. P., Ventura, A. P., Bailey, A. M., Akture, C., OpokuAnsah, J., Celiktas, M., Hwang, M. S., Darling, D. S., Coleman, I. M., Nelson, P. S., Nguyen, H. M., Corey, E., Tewari, M., Morrissey, C., Vessella, R. L., \& Knudsen, B. S. (2011). Metastatic progression of prostate cancer and e-cadherin regulation by zeb1 and SRC family kinases. The American Journal of Pathology, 179, 400-410.

237. Giampieri, S., Manning, C., Hooper, S., Jones, L., Hill, C. S., \& Sahai, E. (2009). Localized and reversible TGFbeta signalling switches breast cancer cells from cohesive to single cell motility. Nature Cell Biology, 11, 1287-1296.

238. Armstrong, A. J., Tannock, I. F., de Wit, R., George, D. J., Eisenberger, M., \& Halabi, S. (2010). The development of risk groups in men with metastatic castration-resistant prostate cancer based on risk factors for PSA decline and survival. European Journal of Cancer, 46, 517-525.

239. Qin, J., Liu, X., Laffin, B., Chen, X., Choy, G., Jeter, C. R., Calhoun-Davis, T., Li, H., Palapattu, G. S., Pang, S., Lin, K., Huang, J., Ivanov, I., Li, W., Suraneni, M. V., \& Tang, D. G. (2012). The PSA(-/lo) prostate cancer cell population harbors selfrenewing long-term tumor-propagating cells that resist castration. Cell Stem Cell, 10, 556-569.

240. Kottke, T., Errington, F., Pulido, J., Galivo, F., Thompson, J., Wongthida, P., Diaz, R. M., Chong, H., Ilett, E., Chester, J., Pandha, H., Harrington, K., Selby, P., Melcher, A., \& Vile, R. (2011). Broad antigenic coverage induced by vaccination with virus-based cDNA libraries cures established tumors. Nature Medicine, 17, 854-859.

241. Kantoff, P. W., Higano, C. S., Shore, N. D., Berger, E. R., Small, E. J., Penson, D. F., Redfern, C. H., Ferrari, A. C., Dreicer, R., Sims, R. B., Xu, Y., Frohlich, M. W., \& Schellhammer, P. F. (2010). Sipuleucel-T immunotherapy for castration-resistant prostate cancer. The New England Journal of Medicine, 363, 411-422.

242. Kantoff, P. W., Schuetz, T. J., Blumenstein, B. A., Glode, L. M., Bilhartz, D. L., Wyand, M., Manson, K., Panicali, D. L., Laus, R., Schlom, J., Dahut, W. L., Arlen, P. M., Gulley, J. L., \& Godfrey, W. R. (2010). Overall survival analysis of a phase II randomized controlled trial of a Poxviral-based PSA-targeted immunotherapy in metastatic castration-resistant prostate cancer. Journal of Clinical Oncology, 28, 1099-1105.

243. Yuan, T. C., Veeramani, S., \& Lin, M. F. (2007). Neuroendocrine-like prostate cancer cells: neuroendocrine transdifferentiation of prostate adenocarcinoma cells. Endocrine-Related Cancer, 14, 531-547.

244. Carver, B. S., Chapinski, C., Wongvipat, J., Hieronymus, H., Chen, Y., Chandarlapaty, S., Arora, V. K., Le, C., Koutcher, J., Scher, H., Scardino, P. T., Rosen, N., \& Sawyers, C. L. (2011). Reciprocal feedback regulation of PI3K and androgen receptor signaling in PTEN-deficient prostate cancer. Cancer Cell, 19, 575-586.

245. Bitting, R. L., \& Armstrong, A. J. (2013). Targeting the PI3K/Akt/ mTOR pathway in castration-resistant prostate cancer. EndocrineRelated Cancer, 20, R83-R99.
246. Glickman, M. S., \& Sawyers, C. L. (2012). Converting cancer therapies into cures: lessons from infectious diseases. Cell, 148, 1089-1098.

247. Goldstein, A. S., Huang, J., Guo, C., Garraway, I. P., \& Witte, O. N. (2010). Identification of a cell of origin for human prostate cancer. Science, 329, 568-571.

248. Cottard, F., Asmane, I., Erdmann, E., Bergerat, J. P., Kurtz, J. E., \& Ceraline, J. (2013). Constitutively active androgen receptor variants upregulate expression of mesenchymal markers in prostate cancer cells. PLoS One, 8, p. e63466.

249. Brennen, W. N., Rosen, D. M., Wang, H., Isaacs, J. T., \& Denmeade, S. R. (2012). Targeting carcinoma-associated fibroblasts within the tumor stroma with a fibroblast activation protein-activated prodrug. Journal of the National Cancer Institute, 104, 1320-1334.

250. Li, Y., Maitah, M. Y., Ahmad, A., Kong, D., Bao, B., \& Sarkar, F. H. (2012). Targeting the Hedgehog signaling pathway for cancer therapy. Expert Opinion on Therapeutic Targets, 16, 49-66.

251. Groth, C., \& Fortini, M. E. (2012). Therapeutic approaches to modulating Notch signaling: Current challenges and future prospects. Seminars in Cell \& Developmental Biology, 23, 465-472.

252. Smith, A. L., Robin, T. P., \& Ford, H. L. (2012). Molecular pathways: Targeting the TGF-beta pathway for cancer therapy. Clinical Cancer Research, 18, 4514-4521.

253. Liu, G., Sprenger, C., Sun, S., Epilepsia, K. S., Haugk, K., Zhang, X., et al. (2013). AR variant ARv567es induces carcinogenesis in a novel transgenic mouse model of prostate cancer. Neoplasia, 15, pp. 1009-1017.

254. Bitting, R. L., \& Armstrong, A. J. (2013). Potential predictive biomarkers for individualizing treatment for men with castrationresistant prostate cancer. Cancer Journal, 19, 25-33.

255. Othus, M., Barlogie, B., Leblanc, M. L., \& Crowley, J. J. (2012). Cure models as a useful statistical tool for analyzing survival. Clinical Cancer Research, 18, 3731-3736.

256. Scher, H. I., Fizazi, K., Saad, F., Taplin, M. E., Sternberg, C. N., Miller, K., et al. (2012). Increased survival with enzalutamide in prostate cancer after chemotherapy. The New England Journal of Medicine, 367, 1187-1197.

257. Clegg, N. J., Wongvipat, J., Joseph, J. D., Tran, C., Ouk, S., Dilhas, A., Chen, Y., Grillot, K., Bischoff, E. D., Cai, L., Aparicio, A., Dorow, S., Arora, V., Shao, G., Qian, J., Zhao, H., Yang, G., Cao, C., Sensintaffar, J., Wasielewska, T., Herbert, M. R., Bonnefous, C., Darimont, B., Scher, H. I., Smith-Jones, P., Klang, M., Smith, N. D., De Stanchina, E., Wu, N., Ouerfelli, O., Rix, P. J., Heyman, R. A., Jung, M. E., Sawyers, C. L., \& Hager, J. H. (2012). ARN-509: a novel antiandrogen for prostate cancer treatment. Cancer Research, 72, 1494-1503.

258. Montgomery, R. B., Eisenberger, M. A., Rettig, M., Chu, F., Pili, R., Stephenson, J., Vogelzang, N. J., Morrison, J., \& Taplin, M. (2012). Phase I clinical trial of galeterone (TOK-001), a multifunctional antiandrogen and CYP17 inhibitor in castration resistant prostate cancer. Journal of Clinical Oncology, 30, abstr 4665.

259. de Bono, J. S., Logothetis, C. J., Molina, A., Fizazi, K., North, S., Chu, L., Chi, K. N., Jones, R. J., Goodman, O. B., Jr., Saad, F., Staffurth, J. N., Mainwaring, P., Harland, S., Flaig, T. W., Hutson, T. E., Cheng, T., Patterson, H., Hainsworth, J. D., Ryan, C. J., Sternberg, C. N., Ellard, S. L., Flechon, A., Saleh, M., Scholz, M., Efstathiou, E., Zivi, A., Bianchini, D., Loriot, Y., Chieffo, N., Kheoh, T., Haqq, C. M., \& Scher, H. I. (2011). Abiraterone and increased survival in metastatic prostate cancer. The New England Journal of Medicine, 364, 1995-2005.

260. Ryan, C. J., Smith, M. R., de Bono, J. S., Molina, A., Logothetis, C. J., de Souza, P., Fizazi, K., Mainwaring, P., Piulats, J. M., Ng, S., Carles, J., Mulders, P. F., Basch, E., Small, E. J., Saad, F., Schrijvers, D., Van Poppel, H., Mukherjee, S. D., Suttmann, H., Gerritsen, W. R., Flaig, T. W., George, D. J., Yu, E. Y., Efstathiou, E., Pantuck, A., Winquist, E., Higano, C. S., Taplin, M. E., Park, Y., Kheoh, T., Griffin, T., Scher, H. I., \& Rathkopf, D. E. (2013). Abiraterone in 
metastatic prostate cancer without previous chemotherapy. The New England Journal of Medicine, 368, 138-148.

261. Kaku, T., Hitaka, T., Ojida, A., Matsunaga, N., Adachi, M., Tanaka, T., Hara, T., Yamaoka, M., Kusaka, M., Okuda, T., Asahi, S., Furuya, S., \& Tasaka, A. (2011). Discovery of orteronel (TAK700), a naphthylmethylimidazole derivative, as a highly selective 17,20-lyase inhibitor with potential utility in the treatment of prostate cancer. Bioorganic \& Medicinal Chemistry, 19, 6383-6399.

262. Yarom, N., Stewart, D., Malik, R., Wells, J., Avruch, L., \& Jonker, D. J. (2013). Phase I clinical trial of Exherin (ADH-1) in patients with advanced solid tumors. Current Clinical Pharmacology, 8, 81-88.

263. Austin, P., Freeman, S. A., Gray, C. A., Gold, M. R., Vogl, A. W., Andersen, R. J., Roberge, M., \& Roskelley, C. D. (2013). The invasion inhibitor sarasinoside A1 reverses mesenchymal tumor transformation in an e-cadherin-independent manner. Molecular Cancer Research, 11, 530-540.

264. Feng, S., Shao, L., Yu, W., Gavine, P., \& Ittmann, M. (2012). Targeting fibroblast growth factor receptor signaling inhibits prostate cancer progression. Clinical Cancer Research, 18, 3880-3888. 


\section{Biologic and Clinical Significance of Androgen Receptor Variants in Castration Resistant Prostate Cancer}

2

3

4

5

6

7

11 *corresponding author:

12 Scott Dehm PhD, Masonic Cancer Center, University of Minnesota, Mayo Mail Code 806, 420

13 Delaware St. SE, Minneapolis, MN 55455 dehm@umn.edu

1) Duke University, 213 Research Dr., 0045 CARL Bldg., Departments of Molecular Genetics and Medicine, and Medicine, Durham NC 27710 USA

3) Masonic Cancer Center, University of Minnesota Masonic Cancer Center, Minneapolis,

\section{Keywords: Androgen receptor, splice variants, castration resistant prostate cancer, AR}

25 variants, metastasis, epithelial to mesenchymal transition

26 Word Count: 7172 words 


\section{Abstract}

29 As prostate cancer progresses to the lethal castration resistant and metastatic form, genetic and

30 epigenetic adaptation, clonal selection, and evolution of the tumor microenvironment contribute

31 to the emergence of unique biologic characteristics under the selective pressure of external

32 stresses. These stresses include the therapies applied in the clinic or laboratory and the

33 exposures of cancers to hormonal, paracrine, or autocrine stimuli in the context of the tumor

34 micro- and macro-environment. The androgen receptor $(A R)$ is a key gene involved in prostate

35 cancer etiology and oncogenesis, including disease development, progression, response to

36 initial hormonal therapies, and subsequent resistance to hormonal therapies. Alterations in the

37 AR signaling pathway have been observed in certain selection contexts and contribute to the

38 resistance to agents that target hormonal regulation of the AR, including standard androgen

39 deprivation therapy (ADT), anti-androgens such as enzalutamide, and androgen synthesis

40 inhibition with abiraterone acetate. One such resistance mechanism is the synthesis of

41 constitutively active AR variants lacking the canonical ligand binding domain. This review

42 focuses on the etiology, characterization, biologic properties, and emerging data contributing to

43 the clinical characteristics of AR variants, and suggests approaches to full-length AR and AR

44 variant biomarker validation, assessment, and systemic targeting in the clinic. 
53

54

55

56

57

58

59

60

61

62

63

64

\section{Introduction}

Prostate cancer (PCa) is the most commonly diagnosed cancer and the second leading cause of cancer related death in men (Siegel 2013). Many of these men are diagnosed with an indolent form of the disease and are candidates for active surveillance rather than immediate therapy, while a minority of men are diagnosed with high grade, high risk disease requiring aggressive surgery, radiation and hormonal therapy approaches. As a result, the risk of death due to prostate cancer is much lower (1 in 30$)$ than the risk of being diagnosed with prostate cancer (1 in 6) and there is an unmet need to identify those who will progress to aggressive disease through the development of predictive biomarkers. For prostate cancer that has disseminated or recurred following primary therapies, current systemic treatments are able to control the disease often for many years. However, the lethal form of metastatic prostate cancer remains an important public health problem, contributing to over 80 deaths per day in the US.

The androgen receptor (AR) has been identified as an important contributor to the etiology and progression of prostate cancer as well as to the initial response and subsequent resistance to endocrine therapies such as androgen deprivation therapy (ADT). AR is a nuclear hormone receptor comprised of 8 exons that make up 4 functional domains including the $\mathrm{NH} 2$ terminal domain (NTD, exon 1), the DNA binding domain (DBD, exons 2 and 3 ), the hinge region (the 3' portion of exon 3 and the 5' portion of exon 4) and the ligand binding domain

73 (LBD, the remainder of exon 4-8) (Figure 1b). The LBD binds androgens leading to the

74 exposure of the nuclear localization signal (NLS) in the hinge region of AR and the translocation

75 of $A R$ to the nucleus. In the nucleus, the DBD is important for recognizing and binding androgen

76 response elements (AREs) in the promoters and enhancers of AR transcriptional targets. When

77 bound to DNA, the AR NTD accounts for the majority of AR transcriptional activity through

78 interactions with co-regulators. 
The androgen/AR axis drives male development, including the genesis and

81 differentiation of the prostate gland. In adult males, the androgen/AR axis continues to be

82 critical for normal prostate gland function. This tissue developmental basis for androgen/AR

83 dependence is also a fundamental property of prostate cancer cells (Garraway and Sellers

84 2006). AR dependence in prostate cancer cells is further reinforced by AR shaping the 3-

85 dimensional architecture of chromatin in the nucleus, enhancing certain inter- and intra-

86 chromosomal interactions that can result in the generation of specific oncogenic fusion proteins

87 that often become androgen-regulated (Berger, et al. 2011; Haffner, et al. 2010; Lin, et al. 2009;

88 Mani, et al. 2009; Weischenfeldt, et al. 2013). For example, fusion of the androgen-regulated

89 TMPRSS2 promoter with the coding region of the ERG transcription factor leads to high-level,

90 androgen-driven overexpression of ERG. These critical roles for AR in maintaining the prostate

91 tissue lineage, as well as expression of the prostate cancer genome are the likely basis for the

92 effectiveness of ADT, which is achieved through orchiectomy or chemically with gonadotropic

93 releasing hormone agonists or antagonists, followed by treatment with anti-androgens or

94 combination approaches. However, despite most men having favorable initial responses to

95 ADT, progression to castration-resistant disease is nearly universal over time. While some men

96 with metastatic disease progress within 1 year of ADT, other men respond 5 or more years,

97 illustrating the clinical heterogeneity in AR targeting (Hussain, et al. 2006), and heterogeneity in

98 survival is even greater in the non-metastatic setting (Freedland, et al. 2007).

Prostate cancer progression on ADT does not typically occur through mechanisms of

101 bypassing androgen or AR dependence, as evidenced by studies demonstrating the importance

102 of autocrine androgen synthesis and AR protein overexpression for CRPC (Chen, et al. 2004;

103 Stanbrough, et al. 2006). Aberrant AR transcriptional activity in CRPC can occur due to

104 amplification of the $A R$ gene leading to protein overexpression (Chen et al. 2004), mutations in

105 the NH2- domain (NTD) or ligand binding domain (LBD) that render the receptor more sensitive 
106 to androgen activation (Han, et al. 2001) or induce inappropriate agonist responses to

107 antagonists such as bicalutamide or enzalutamide (Balbas, et al. 2013; Joseph, et al. 2013;

108 Korpal, et al. 2013). In addition, the de novo autocrine synthesis of androgens by the tumor itself

109 can occur through overexpression of key androgenic metabolic enzymes such as CYP17A1,

110 AKR1C3, HSD3B2, CYP11A1, and SRD5A1 and 2 (Mostaghel, et al. 2011), or through

111 mutational activation of these enzymes, such as the recently described protein-stabilizing

112 mutation in HSD3B2 (Chang, et al. 2013). Finally, ligand-independent AR activity is modulated

113 by post-translational modifications, including phosphorylation, SUMOlyation, methylation,

114 ubiquitination, and acetylation. Each of these protein modifications is regulated by upstream

115 oncogenic events and may converge on the AR to support persistent activity (Coffey and

116 Robson 2012).

$118 \quad$ Further evidence of sustained AR activity was demonstrated by the responsiveness and

119 survival benefits observed with newer AR-targeted agents such as enzalutamide and

120 abiraterone acetate, which were developed to more potently inhibit AR signaling in the face of

121 castrate levels of testosterone (de Bono, et al. 2011; Ryan, et al. 2013; Scher, et al. 2010;

122 Scher, et al. 2012). Abiraterone acetate is a CYP17 hydroxylase and lyase inhibitor, which

123 partially inhibits androgen synthesis in the adrenal gland, testes and tumor tissue. Abiraterone

124 increased overall survival from 10.9 months to 14.8 months in the post-docetaxel metastatic

125 CRPC setting (de Bono et al. 2011), and delayed progression or death by over 8 months in the

126 pre-chemotherapy metastatic CRPC setting (Ryan et al. 2013). Enzalutamide binds AR with

127 higher affinity than conventional antiandrogens and impairs AR nuclear localization and

128 transcriptional activity even under conditions of AR overexpression (Clegg, et al. 2012; Tran, et

129 al. 2009). Enzalutamide increased median overall survival from 13.6 months in the placebo

130 group to 18.4 months in the enzalutamide group in the post-docetaxel metastatic CRPC setting

131 (Scher et al. 2012), and a phase 3 trial in the pre-docetaxel metastatic CRPC setting has been 
132 completed with results anticipated soon. The greater activity of these agents when used earlier

133 in the disease suggests the relative importance of these endocrine/autocrine resistance

134 mechanisms in early CRPC development.

135

However, despite the initial striking response to these next generation AR-targeted

137 agents in the clinic, resistance develops typically within 1-2 years in nearly all men with

138 metastatic CRPC, as evidenced by rises in PSA or radiographic and symptomatic signs of

139 progressive tumor growth or dissemination. Mutations in AR leading to enzalutamide agonism

140 suggest that the selection pressure of potent AR inhibition leads to tumor adaptation or selection

141 of clones able to persist despite enzalutamide (Balbas et al. 2013; Joseph et al. 2013; Korpal et

142 al. 2013). These data also suggest the central importance of AR in CRPC biology, given that

143 this selection pressure and emergence of resistant mutations would not be expected to occur if

144 AR was not a central regulator of prostate cancer survival at least in some men with CRPC.

145 While the cell of origin in human PCa is debated and may be heterogeneous in different

146 contexts (Choi, et al. 2012; Goldstein, et al. 2010; Wang, et al. 2009; Wang, et al. 2013), these

147 data suggest that AR plays an important role in regulating the survival of the bulk of prostate

148 cancer cells in many patients. In addition, cross-resistance is commonly observed between

149 enzalutamide and abiraterone acetate in the clinic (Loriot, et al. 2013; Noonan, et al. 2013;

150 Schaeffer 2013). This "one and done" nature of treatment response suggests that there are

151 underlying mechanisms of resistance to both agents that limit subsequent treatment with the

152 other. This cross-resistance is likely mediated at least in part by alterations in AR structure and

153 function, given that progressive disease is typically accompanied by continued rises in serum

154 levels of PSA, an AR-regulated gene. In further support of this, multiple constitutively active AR

155 variants have been shown to be expressed in CRPC. These AR variants lack the AR LBD, the

156 domain through which conventional ADT, abiraterone, and enzalutamide all exert their inhibitory

157 actions. However, the precise role of AR variants in mediating prostate cancer progression is 
158 still being deliberated. Herein, we will review the accumulating evidence pertaining to AR variant

159 characterization, biologic properties, emergence in the clinic, and the clinical implications of AR

160 variants in mediating lethal CRPC

162 A. Origin of Androgen Receptor Variants in Prostate Cancer

163 Over the past decade, 17 AR variants that lack the ligand-binding domain have been

164 discovered in prostate cancer cell lines (22Rv1, VCaP and CWR-R1), human xenografts

165 (LuCaP 86.2, LuCaP136) and clinical prostate cancer specimens. AR variants share a common

166 core NTD/DBD structure encoded by contiguously-spliced AR exons 1, 2, and 3, but differ at

167 their $\mathrm{COOH}$-terminal end by virtue of being encoded by mRNAs with different 3' terminal exons

168 (Figure 1b). The most striking feature of AR variants is the lack of the LBD, which strongly

169 suggests they play a role in prostate cancer progression and resistance to therapies targeted at

170 this domain. Of the $17 \mathrm{AR}$ variants that have been reported, 10 have been reliably detected by

171 RT-PCR in prostate cancer specimens (Table 1). However, the levels of certain AR variants in

172 these clinical specimens appear to be highly variable, and are usually lower than in the cell lines

173 and xenograft models in which they were originally discovered. (Ceraline, et al. 2003; Dehm, et

174 al. 2008; Guo, et al. 2009; Hu, et al. 2009; Hu, et al. 2011; Sun, et al. 2010; Watson, et al. 2010;

175 Yang, et al. 2011; Zhang, et al. 2011) (Table 1). Several mechanisms for the synthesis of AR

176 variants have been identified, including proteolytic cleavage of full-length $A R$, genomic

177 alterations/mutations, and alternative or altered splicing. These mechanisms are discussed in

178 detail below.

180 Proteolytic Cleavage

181 An AR variant termed trAR was shown to result from cleavage by calpains (calcium-

182 dependent proteinases) (Libertini, et al. 2007). Calpains can cleave the full-length AR protein in

183 the hinge region between amino acids 648 and 649 and generate two polypeptides: an 80-kDa 
184 protein consisting of the NTD, DBD, and hinge domains, and a second protein containing the

185 LBD. 22Rv1 is an androgen-independent cell line derived from the androgen-dependent parent

186 CWR22 tumor xenograft and is the cell line in which an AR variant protein lacking the LBD was

187 first discovered (Tepper, et al. 2002). Treatment of 22Rv1 cells with a calpain inhibitor reduced

188 AR variant expression and re-sensitized cells to ADT (Libertini et al. 2007) indicating trAR

189 synthesis is partially calpain-dependent, post-translationally regulated, and important for

190 androgen-independence. Further, calpain expression was increased in metastatic prostate

191 cancer (Rios-Doria, et al. 2004), but there is no direct evidence of calpain mediated AR variant

192 synthesis in prostate cancer specimens.

193

194 Point Mutations and Genomic Rearrangements

195 An early study used a yeast-based functional assay to detect and analyze mutant ARs in

196 prostate cancer. In this study, Ceraline et al. discovered a nonsense mutation located in exon 4,

197 Q640X, that creates a premature stop codon, leading to synthesis of a constitutively active AR

198 variant protein that localizes to the nucleus (Bergerat and Ceraline 2009; Ceraline et al. 2003).

199 More recently, genomic alterations within the $A R$ gene have been shown to generate AR

200 variants (Li, et al. 2011; Li, et al. 2012; Nyquist, et al. 2013). The first genomic alteration (a 35kb

201 tandem duplication encompassing exon 3 and flanking cryptic exons) (Figure 1a) was

202 discovered in 22Rv1 cells (Li et al. 2011). Although calpain-mediated proteolysis was thought to

203 be the primary mechanism leading to AR variant expression in $22 \mathrm{R} v 1$, multiple subsequent

204 studies have shown that altered splicing is the dominant force. The discovery of this $35 \mathrm{~kb}$

205 tandem duplication in 22Rv1 provided a rational basis for the altered splicing observed in these

206 cells. Importantly, culture of CWR22Pc cells (an androgen-dependent cell line derived from the

207 original CWR22 xenograft) in androgen-depleted media led to the detection of the $35 \mathrm{~kb}$ tandem

208 duplication in the $A R$ gene and expression of $A R$ variant protein. This suggests that expression

209 of AR variants in CWR22Pc cells is limited to those cells harboring the genetic duplication and 
210 that these genomic alterations cause variations in AR splicing. Indeed, AR intragenic

211 rearrangements have also been defined in human xenograft models LuCaP 86.2 and LuCaP

212136 (8.5kb deletion or 8.7kb inversion including exons 5-7) (Figure 1a), which both express

213 high levels of the AR-V12/AR ${ }^{\mathrm{v567es}}$ variant lacking exons 5, 6 and 7 (Li et al. 2012; Nyquist et al.

214 2013). Interestingly, these gene rearrangements in LuCaP 86.2 and 136 result in $A R$ gene

215 structures that can only produce AR variant. It is also noteworthy that $A R$ gene rearrangements

216 do not always encompass alternatively spliced exons. For example, a 48kb deletion located in

217 intron 1 of the AR gene was identified in a sub-population of cells in the CWR-R1 cell line.

218 CWR-R1 cells express high levels of AR-V7 arising from exon 3 splicing to the cryptic exon CE3

219 located in AR intron 3 (Li et al. 2012) (Figure 1b). Because the "alternatively spliced" exon CE3

220 is not directly affected by the $A R$ gene rearrangement in these cells, it is not immediately clear

221 how this genomic event could favor AR-V7 synthesis. However, there does appear to be a

222 direct link between the CWR-R1 cell population harboring this deletion and altered AR splicing.

223 For instance, culturing CWR-R1 cells long-term under castrate conditions leads to outgrowth of

224 the sub-population harboring the AR intron 1 deletion as well as increased expression of AR-V7.

225 In addition, single cell cloning demonstrated that high-level AR-V7 expression was restricted to

226 cells positive for this deletion ( $\mathrm{Li}$, et al. 2013). Together, these data indicate that distant changes

227 in the $A R$ genomic architecture can cause splice switches that favor the expression of $A R$

228 variants.

229

230 The mechanism for genomic rearrangements is thought to be induced by the structural

231 architecture associated with the local genomic region (Gu, et al. 2008). The AR intragenic

232 duplications, deletions, and inversions identified in prostate cancer contain signatures of

233 microhomology-mediated break induced replication (MMBIR) or non-homologous end joining

234 (NHEJ) at break fusion junction sites (Li et al. 2011; Li et al. 2012; Nyquist et al. 2013). The

235 presence of long interspersed nuclear elements (LINE-1) flanking the fusion, microhomology at 
236 the breakpoint, and inserted sequence at the fusion in 22Rv1 cells are trademark for MMBIR 237 mechanisms (Hastings, et al. 2009). The diverse genomic architecture of the proximal arm of

238 the $\mathrm{X}$ chromosome is characterized by a high concentration of recombinogenic structures that

239 can form hairpins and cruciforms and render it ideal for replication-based rearrangements; thus,

240 leading to complex somatic rearrangements seen in cancer. Furthermore, MMBIR and NHEJ

241 catalyze non-recurrent breakpoints (Koumbaris, et al. 2011). Therefore, the exact locations of

242 AR breakpoints are expected to vary between patient specimens giving intrinsic genomic

243 heterogeneity.

245 The functional significance of $A R$ genomic alterations was addressed in a study

246 implementing transcription activator-like effector nuclease (TALEN) genome engineering to

247 model the rearrangements observed in LuCaP 86.2 and LuCaP 136. TALENs are synthetic

248 fusion proteins consisting of a non-specific DNA-cleaving nuclease and a DNA-binding domain

249 that is engineered to target a sequence of interest (Joung and Sander 2013). Using TALENs

250 targeted to exons 4 and 7, cell lines were generated with deletion or inversion events

251 comprising AR exons 5-7, thus modeling the rearrangements naturally-occurring in LuCaP 86.2

252 and LuCaP 136 xenografts. These engineered rearrangements produced cells that expressed

253 AR-V12/AR ${ }^{\mathrm{v} 567 e s}$ exclusively, thereby creating the first AR variant-dependent model that is

254 completely androgen-independent. Further, in a heterogeneous cell population, ADT enriched

255 for the growth of cells containing the $A R$ deletion or inversion. Importantly, AR-V12/AR ${ }^{\mathrm{v} 567 e s}$ was

256 able to maintain constitutive activity of the AR transcriptional program. This investigation was

257 the first to verify that the $A R$ gene rearrangements encompassing exons 5-7 are causal events

258 that lead to expression of $A R-V 12 / A R^{v 567 e s}$ and that a constitutively active AR variant is capable

259 of sustaining the AR transcriptional program in an endocrine-independent manner.

261 Alternative Splicing 
It is clear from the studies discussed above that rearrangements in the $A R$ gene underlie

263 splice switches that lead to the synthesis of AR variants; however, other reports indicate that AR

264 variants can also be induced rapidly in response to ADT. Ligand-bound AR binds to an ARE

265 located in intron 1 of the $A R$ gene and recruits repressive lysine-specific demethylase (LSD1)

266 activity, leading to AR-mediated feedback inhibition of AR transcriptional activity. ADT relieves

267 this feedback inhibition, increasing AR transcriptional output and expression of full-length AR

268 and possibly AR variants (Cai, et al. 2011). In line with this, a series of preclinical studies

269 demonstrated that AR-V7 mRNA expression is rapidly upregulated subsequent to ADT and can

270 be reversed when full-length AR signaling is reactivated (Hu, et al. 2012; Watson et al. 2010).

271 These data indicate that AR-V7 expression is acutely and reversibly regulated, perhaps by way

272 of androgen-regulated changes in AR transcriptional output, AR alternative splicing or both. The

273 rapid reversibility of these reactions suggest that expression of AR-V7 in this case cannot be

274 accounted by clonal selection of cell populations with $A R$ gene rearrangements.

275

The importance of AR transcriptional elongation rates for increased AR-V7 expression

277 was recently demonstrated by Liu et al, who detected increased expression of both full-length

278 AR and AR-V7 following ADT. Higher AR transcription rates were required to increase the

279 recruitment of the necessary splicing factors for global increases in mature AR mRNA species.

280 The authors concluded that ADT enhances $A R$ transcription, which leads to increased AR pre-

281 mRNA that is processed through splicing to generate mRNAs encoding AR-V7 as well as full-

282 length AR. Importantly, this study also identified two cis-acting elements (one intronic splicing

283 enhancer and one exonic splicing enhancer) near the 3' splice site of AR exon CE3 (also

284 referred to as AR exon 3b, the cryptic exon spliced to exon 3 in AR-V7, see Figure 1b) and the

285 respective trans-acting binding proteins (U2AF2 and SFRS1) (Liu, et al. 2013b). The

286 mechanism by which these splicing factors increase association with the AR mRNA following

287 ADT is not known. They are unlikely to be direct transcriptional targets of AR variants as there 
288 was no change in their expression after treatment. To date, this is the only study analyzing 289 plasticity in AR variant expression via an alternative splicing mechanism. Future effort is needed 290 to address if these splice factors are important for the splicing of other AR variants and whether

291 these cis- and trans-factors are important in the context of $A R$ gene rearrangements occurring in 292 cell lines, tumor xenografts, and clinical CRPC specimens. In cells such as CWR-R1, where

293 large AR gene rearrangements occur but all AR exons are present in their normal order, it will

294 be important to understand the role of the splicing machinery in recognizing alternative splice

295 sites and supporting AR variant synthesis. Also, additional cis-acting elements and trans-acting

296 factors need to be mapped and identified for the additional cryptic exons that have been shown

297 to splice into AR mRNAs and lead to synthesis of other AR variants.

Overall, these studies have demonstrated that diverse genetic and epigenetic

300 mechanisms can mediate expression of AR variants, leading to androgen-independent

301 activation of the AR pathway. Cells with AR gene rearrangements display stable and efficient

302 synthesis of AR variants (Li et al. 2011; Li et al. 2012). In contrast, cells that regulate AR

303 variants acutely in response to ADT express much lower levels of AR variant compared to full-

304 length AR (Hu et al. 2012; Watson et al. 2010). Based on these considerations, it is tempting to

305 speculate that AR variants are synthesized acutely to survive ADT conditions, and that the $A R$

306 gene is a hotspot for genomic rearrangements eventually selecting for arrangements that can

307 support stable, efficient synthesis of AR variants and promote prostate cancer progression. In

308 addition, the prevalence and abundance of AR variants is likely context-dependent, varying and

309 increasing over time as prostate cancer progresses from localized disease to metastatic disease

310 to CRPC and eventually to enzalutamide/abiraterone refractory metastatic CRPC.

312 B. Role of AR variants in CRPC Progression

\section{Preclinical Models}


314 Previous studies demonstrated that the specific knockdown of endogenous AR variants

315 in androgen-independent cell lines, 22Rv1 and CWR-R1, was sufficient to inhibit both in vitro

316 and in vivo growth under castrate conditions (Guo et al. 2009; Li et al. 2012), as well as restore

317 cell responsiveness to androgens and enzalutamide (Li et al. 2013). In addition, overexpression

318 of AR variants was sufficient to drive growth during ADT in androgen-dependent LNCaP cells

319 (Chan, et al. 2012; Dehm et al. 2008; Guo et al. 2009; Sun et al. 2010). However, there have

320 been conflicting reports as to whether AR variants provide an overall growth advantage, or are

321 only advantageous to tumor cells under hormone-depleted conditions. On one hand, xenografts

322 of LNCaP cells expressing the AR-V12/AR ${ }^{\mathrm{v} 567 e s}$ variant grew larger than LNCaP xenografts

323 alone, but only after castration (Sun et al. 2010). Conversely, a genomic mouse model

324 engineered to express AR-V12/AR ${ }^{\mathrm{v567es}}$ only in prostate epithelium demonstrated that both sham

325 and castrated mice had a significant increase in GU and prostate weight compared to WT

326 controls. This indicates that $A R-V 12 / A R^{v 567 e s}$ can support higher proliferative rates in the

327 prostate epithelium even prior to castration-induced ADT, likely by augmenting full-length AR

328 activity. On the other hand, the function of $A R-V 12 / A R^{v 567 e s}$ in advanced progression to

329 adenocarcinoma is more pronounced in castrated AR-V12/AR ${ }^{\mathrm{v} 567 \mathrm{es}}$ mice compared with sham

330 treated AR-V12/AR ${ }^{\mathrm{v567es}}$ mice (Liu, et al. 2013a). In an AR-V7 genetically engineered mouse

331 model, mice expressing AR-V7 had impaired prostate regeneration after re-administration of

332 testosterone following castration (Sun, et al. 2013). These data suggest that AR variants may

333 have a functional role in normal prostate epithelium that is deregulated when expression of AR

334 variants are selected for during ADT, or that ADT itself regulates a set of genes that alter AR

335 variant activity and function. Given that high AR variant expression in bone metastases is

336 associated with shorter time to death in men with metastatic CRPC (Hornberg, et al. 2011), and

337 that high levels of AR variant expression in localized tumors is associated with disease

338 recurrence (Guo et al. 2009), AR variant expression is likely to be important across the disease

339 spectrum of aggressive prostate cancer, particularly during ADT resistance (Figures 2c and d). 
Clinical Impact of AR Variant Emergence

342 Studies of AR variants in the clinic are limited by the lack of clinically validated assays

343 for individual AR variant detection using specific antibodies and the dearth of large metastatic

344 tissue repositories linked to CRPC outcomes. Despite these limitations, which are currently

345 being addressed, current evidence suggests that AR variants may be functional drivers of

346 prostate cancer progression (Guo et al. 2009; Hornberg et al. 2011; Hu et al. 2009; Hu et al.

347 2011; Sun et al. 2010; Zhang et al. 2011). Hu et al. (Hu et al. 2009) focused on AR-V1 and AR-

348 V7 expression and found an average 20-fold higher expression of variant mRNA in castration

349 resistant prostate cancer (CRPC) $(n=25)$ than in hormone-naïve $(H N)$ PCa $(n=82, P<0.0001)$.

350 AR-V7 mRNA, but not AR-V1 mRNA, predicted biochemical recurrence following surgical

351 treatment $(P=0.012)$ (Table 2), suggesting that not all $A R$ variants are equivalent in predicting

352 clinical resistance and progression. Guo and colleagues (Guo et al. 2009) assessed AR-V7

353 protein expression by immunohistochemistry in castration-resistant, $\mathrm{HN}$ and benign tumor

354 samples. Nuclear AR-V7 staining was positive in $44 \%$ of castration-resistant samples compared

355 to $9 \%$ and $0 \%$ in $\mathrm{HN}$ and benign samples, respectively. This study further evaluated the

356 predictive value of AR-V7, leading to the unexpected finding that high cytoplasmic staining of

357 AR-V7 had a greater risk for PSA recurrence after radical prostatectomy $(P<0.0001)$, and high

358 AR-V7 staining had the highest hazard ratio $(2.486, P<0.0043)$ for prostate cancer recurrence

359 (Figure 2d, Table 2). The functional significance of AR-V7 cytoplasmic expression is not clear;

360 particularly in light of the finding that AR-V7 has a functional NLS-like sequence that enhances

361 efficient nuclear localization (Chan et al. 2012). In contrast to these studies that found AR

362 variant levels were predictive of clinical outcome, a separate study that analyzed mRNA levels

363 of AR-V1 and AR-V7 in surgical specimens using branched chain technology found that there

364 was no association between AR variant expression and disease recurrence (Zhao, et al. 2012).

365 The difference in AR-V7 mRNA association with disease recurrence between this study and the 
366 study by Hu et al (Hu et al. 2009) could be due to the methods of measuring AR-V7 (branched

367 chain vs. RT-PCR) in prostate specimens.

There is evidence that AR variants are expressed at low mRNA levels relative to full-

370 length AR in both benign and malignant epithelium. Although AR variants are detectable in

371 benign/normal tissues, their possible functional roles in normal prostate physiology and

372 embryogenesis or regeneration are not known. It is possible that AR variants may not play a

373 role in driving tumor initiation, but instead play a specific functional role in driving tumor

374 progression during ADT. In support of this hypothesis, analysis of $A R-V 12 / A R^{v 567 e s}$ and $A R-V 7$

375 mRNA expression in metastatic samples from CRPC patients revealed that out of 46 AR-

376 positive metastases, $43 \%$ expressed $A R-V 12 / A^{v 567 e s}$ and $24 \%$ expressed $A R-V 7$ mRNA

377 compared to $17 \%$ and $6 \%$ in normal tissue, respectively (Sun et al. 2010). Another study

378 focusing on bone metastases found that full-length AR, AR-V7, and AR-V1 mRNAs were

379 detected in $100 \%$ of samples $\left(n=30\right.$ bone metastases) and AR-V12/AR ${ }^{\text {v567es }}$ mRNA was

380 detected in $23 \%$ of samples. AR-V1 and AR-V7 mRNAs were also detectable in non-malignant

381 prostate tissue, primary prostate tumors, and hormone-naïve bone metastases. Nonetheless,

382 both the number of AR variant positive samples and the mRNA levels of AR variants increased

383 with disease progression (Table 2), and cancer-free survival after metastasis surgery was

384 significantly shorter in patients with high AR-V7 mRNA expression (Figure 2c) (Hornberg et al.

385 2011). Finally, Zhang et al. (Zhang et al. 2011) measured variations in N-and C-terminal AR

386 expression in primary and metastatic PCa tissues and found a significant decrease in nuclear C-

387 terminal AR staining relative to $\mathrm{N}$-terminal AR staining in metastatic samples, indicating the loss

388 of full-length AR and enrichment for AR variants (Figure $2 \mathbf{a}$ and $\mathbf{b}$ ) during the metastatic

389 process. 
392 progression in large tissue collections is still being elucidated; however, the correlation between

393 AR variant expression and CRPC is highly suggestive that these variants are selected to drive

394 resistance during treatment. This notion is further supported by the existence of specific $A R$

395 gene rearrangements in clinical CRPC tissues that can cause AR variant expression and

396 therapeutic resistance (Nyquist et al. 2013). Likewise, alternative AR-dependent and AR-

397 independent resistant mechanisms will also exist. No current data are available from clinical

398 CRPC tissues wherein AR variant expression has been measured pre- and post enzalutamide

399 or abiraterone treatment, but phase II clinical trials (NCT01942837, NCT01503229,

400 NCT01385293) are ongoing to directly address this subject. Based on the available data, it is

401 clear that high levels of AR variant expression can confer resistance to second-generation

402 antiandrogens and inhibitors targeting androgen synthesis, particularly when AR gene

403 rearrangements underlie this expression. However, it remains to be established whether AR

404 variants can confer resistance when expressed at the low levels found in benign epithelium or

405 hormone-naïve PCa. A barrier to addressing this question is a lack of validated and reliable

406 clinical laboratory assessments for individual AR variants on collected metastatic tumor tissue,

407 circulating tumor cells, or in circulating plasma RNA that may permit the longitudinal

408 assessment of AR variant emergence and targeting in the clinic.

410 C. Biological Functions of AR Variants Contributing to CRPC

\section{Transcriptional Programs}

412 The precise role of the AR variants in the progression of men with CRPC remains

413 incomplete until interrogation of all possible AR variants can be performed using unbiased

414 approaches and linked to outcomes independent of other known AR alterations and oncogenic

415 events that drive CRPC. However, from model systems it is clear that the AR variants are

416 capable of recapitulating the full-length AR transcriptional program in the absence of androgens 
417 or full-length AR (Hu et al. 2012; Liu et al. 2013b; Nyquist et al. 2013), and several studies have 418 also identified transcriptional targets that may be distinct to AR variants (Hu et al. 2012; Liu et 419 al. 2013b; Sun et al. 2010). For example, Hu et al. identified gene sets that were upregulated 420 during overexpression of $A R-V 7$ and $A R-V 12 / A R^{v 567 e s}$, including several cell cycle related

421 genes. Additionally, AR-V7 expression correlated with expression of one of these cell cycle 422 genes, UBE2C, in prostate specimens biopsied after hormone therapy (Hu et al. 2012). These

423 data suggest that AR variants mediate a distinct cell cycle gene expression profile that is

424 separate from full-length AR. In support of these data, several other studies have found 425 upregulation of cell cycle genes in samples with AR variant expression (Hornberg et al. 2011;

426 Liu et al. 2013b; Zhang et al. 2011). However, induction of this set of cell cycle related genes

427 might simply be an indicator of a pro-proliferative level of AR transcriptional output. For

428 instance, one study found that full-length AR signaling was also able to stimulate a

429 transcriptional signature enriched for the AR-V7 signature cell cycle related genes, but only at

430 low, pro-proliferative androgen concentrations, while high, anti-proliferative androgen

431 concentrations promoted a transcriptional signature that was negatively enriched for the cell

432 cycle gene set. Moreover, increasing levels of exogenous AR-V7 and AR-V12/AR ${ }^{\mathrm{v} 567 e s}$

433 expression led to a similar dichotomy in positive and negative regulation of these cell cycle

434 genes (Li et al. 2013). Therefore, the strength of transcriptional output from full-length AR or AR

435 variants appears to dictate whether this distinct set of cell cycle related genes is activated or

436 repressed. These findings may have importance, as they may indicate that therapies inhibiting

437 the cell cycle and/or proliferation may be effective for the treatment of CRPC (Table 3).

In contrast to AR variants that display constitutive transcriptional activity, a plasma

440 membrane associated AR variant, AR8 (which we propose be termed AR-V15), is predicted to

441 encode a protein containing only the NTD and an unique 33- amino acid C-terminus. AR8

442 increased EGF-induced transcriptional activation of full length AR through the recruitment of AR 
443 to the plasma membrane for phosphorylation. This data suggest AR8 may function in

444 potentiating the AR transcriptional program in response to growth factors during ADT. Moreover,

445 AR8 specific knockdown inhibited androgen-independent growth in cell lines and was expressed

446 in both benign prostate and tumor samples. However, more clinical evidence is needed to

447 signify the importance of AR8 in CRPC (Yang et al. 2011).

AR Variant Induction of an Epithelial to Mesenchymal Transition (EMT) and Invasiveness In response to ADT, prostate cancer xenografts have been found to display an acute

451 and reversible up-regulation of both mesenchymal markers (Sun, et al. 2012) and AR variants

452 (Hu et al. 2012; Watson et al. 2010). These studies, combined with evidence demonstrating

453 increased AR variant expression in metastatic prostate cancer specimens, suggest that there is

454 a relationship between AR variant signaling and an epithelial-to-mesenchymal transition (EMT)

455 program. This epithelial plasticity may be critical for prostate cancer metastatic dissemination 456 and treatment resistance (Bitting, et al. 2014). Additionally, the splicing factor SFRS1, which

457 was identified to be important for AR-V7 splicing, is known to promote EMT by inducing splicing 458 of a constitutively active isoform of Macrophage Stimulating 1 Receptor (MST1R; aka RON1) 459 (Ghigna, et al. 2005). MST1R is known to be overexpressed in prostate cancer and is involved 460 in invasiveness and tumor-microenvironmental interactions in prostate cancer progression 461 (Gurusamy, et al. 2013). It is tempting to speculate that AR variant synthesis and EMT are dual 462 outcomes of an alternative splicing program induced by ADT that could be targeted in CRPC 463 (Table 3). Alternatively, EMT may be important in AR independent PCa (Tanaka, et al. 2010), 464 based on the inverse relationship observed between EMT biomarkers such as N-cadherin and 465 AR expression in CRPC metastatic specimens. Expression of EMT biomarkers is common in 466 circulating tumor cells (CTCs) of men with metastatic CRPC, suggesting that this epithelial 467 plasticity and up-regulation of mesenchymal and stemness biomarker expression may be 468 relatively common in lethal CRPC, regardless of AR activity (Armstrong, et al. 2011). 
470 In support of a possible link between AR variant signaling and EMT, AR variants have

471 been implicated in driving a mesenchymal and invasive phenotype. For example,

472 overexpression of AR variants Q640X and AR-V7 in LNCaP cells resulted in upregulation of N-

473 cadherin mRNA and protein compared to full-length AR, and activation of full-length AR

474 signaling reduced $\mathrm{N}$-cadherin expression (Cottard, et al. 2013). N-cadherin up-regulation

475 coincided with increased mRNA expression of several other mesenchymal markers, including

476 vimentin, Snail, and Zeb1 in cells transfected with AR variants. However downregulation of the

477 epithelial marker E-cadherin was not observed (Cottard et al. 2013). Another study identified

478 Ras Homolog Gene Family Member B (RHOB), a small GTPase, as a distinct target gene

479 activated by a "hybrid" AR variant, termed TC-AR, which was modeled after the Q640X mutant

480 form of AR containing two copies of AR exon 3 (Tsai, et al. 2012). Interestingly, RHOB

481 increased migration and morphological changes in cells expressing TC-AR; however, it did not

482 contribute to androgen-independent growth (Tsai et al. 2012). Given the role of Ras pathway

483 overexpression in CRPC development and EMT biology (Mulholland, et al. 2012,; Taylor, et al.

484 2010) future studies are needed to address the functional significance of increased

485 mesenchymal markers on the metastatic potential of these cells. It is likely these cells will have

486 increased tumorigenicity based on another recent preclinical study that generated a genetically

487 engineered mouse model in which $A R-V 12 / A R^{v 567 e s}$ expression was under the control of the

488 prostate-specific probasin promoter. Importantly, well-differentiated prostate adenocarcinoma

489 formed with high penetrance in AR-V12/AR ${ }^{\mathrm{v} 567 e s}$ mice, and tumors expressed increased Twist

490 and vimentin expression and decreased E-cadherin expression compared to normal epithelium

491 of wild type mice. This further supports the idea that EMT may be one of the mechanisms

492 underlying the invasive phenotype of tumors expressing the AR-V12/AR ${ }^{v 567 e s}$ variant (Liu et al.

493 2013a). Additionally, a genetically engineered mouse model expressing AR-V7 demonstrated a

494 similar increase in mesenchymal markers, as well as stemness biomarkers in comparison to 
495 wild type mice (Sun et al. 2013). In summary, expanding preclinical data demonstrates that AR

496 variants activate traditional AR targets, and possibly additional distinct targets related to the cell

497 cycle, cancer invasiveness, and EMT. These putative novel downstream targets of AR variant

498 activation may represent therapeutic targets in CRPC (Table 3).

500 Enhancers and Inhibitors of AR Variants

501 Efforts are also underway to identify strategies to inhibit AR variant activity through the

502 prevention of nuclear localization, dissociation from co-activators, or by enhancing AR

503 degradation. AR pioneering factors and co-activators are numerous and have been reviewed

504 extensively elsewhere (Shiota, et al. 2011; van de Wijngaart, et al. 2012). Recent studies

505 suggest important disease-relevant roles for ERG, PARP, Rb, FOXA1, and EZH2 in regulating

506 transcriptional activity of full-length AR (Chen, et al. 2013; Schiewer, et al. 2012; Sharma, et al.

507 2010; Xu, et al. 2012). These emerging data suggests a complex interplay between oncogenic

508 signaling, epigenetic regulation, and AR activity, which may also extend to the activity of AR

509 variants. Most AR variants lack the canonical nuclear localization signal (NLS) in the AR hinge

510 region. However, even with loss of the NLS, the NTD/DBD core common to all AR variants has

511 an inherent property of localizing efficiently to the nucleus, although the precise mechanisms for

512 this are not clear (Chan et al. 2012). For certain AR variants, such as AR-V12/AR ${ }^{\mathrm{v} 567 e s}$ and AR-

$513 \mathrm{V7}$, this inherent localization guided by the NTD/DBD core is further enhanced by their unique

$514 \mathrm{COOH}$-terminal extensions that harbor the AR NLS or recapitulate NLS-like sequences. The

515 loss of the AR LBD also disrupts the interaction of AR variants with the chaperone protein heat

516 shock protein 90 (HSP90) in the cytoplasm. Studies involving HSP inhibitors have varied in their

517 abilities to affect AR variant activity (Chan et al. 2012; Gillis, et al. 2013; Shafi, et al. 2013). In

518 LNCaP cells engineered to overexpress AR-V7, two independent HSP90 inhibitors

519 (Geldanamycin and MJC13) failed to inhibit AR variant stabilization or localization to the nucleus

520 (Shafi et al. 2013). In an independent study, another HSP90 inhibitor (AUY922) failed to inhibit 
521 nuclear import of AR variants, and no AR variant:HSP90 complex could be detected.

522 Surprisingly, despite the independence of AR variants from HSP90, cells lines positive for AR

523 variants displayed growth sensitivity to the HSP90 inhibitor (Gillis et al. 2013). Moreover,

524 overexpression of AR variants in LNCaP cells was not sufficient to render them resistant to the

525 HSP90 inhibitor (Gillis et al. 2013). These data indicate that HSP90 inhibitors may have clinical

526 application in CRPC (Table 3) that is independent of AR variant expression.

527

528

529

530

531 length AR signaling (Oh, et al. 2012). When full-length AR or AR Q640X was expressed in AR-

532 null PC3 cells sorafenib treatment inhibited transcriptional activity of both full-length and AR

533 Q640X via proteasomal degradation, with no effect on nuclear localization. Furthermore,

534 sorafenib inhibited proliferation in both AR negative (DU145, PC3) and AR positive (LNCaP,

535 22Rv1) cells, with significantly more inhibition in AR positive cells (Zengerling, et al. 2012). In

536 further support of an important role for AR variant regulation by phosphorylation, a recent report

537 found that DAB2IP is capable of preventing both full-length AR and AR variant nuclear

538 localization through dual mechanisms of inhibiting c-src phosphorylation of AR S81 and PP2A-

539 mediated S81 dephosphorylation (Wu, et al. 2013). Importantly, loss of DAB2IP has been linked

540 to invasive carcinoma in CRPC by suppressing RAS activity and EMT through NFkB in prostate

541 cancer (Chen, et al. 2002; Min, et al. 2010). These studies show that DAB2IP is an inhibitor of

542 RAS signaling, EMT, full-length AR activity, and AR variant activity. Therefore, DAB2IP may

543 serve as a potential therapeutic target for the treatment of CRPC. Planned studies of combined

544 inhibition of AR signaling and $\mathrm{EZH} 2$, the prime inhibitor of DAB2IP, should address this complex

545 functional interplay (Table 3).

546 
An alternative strategy for inhibiting activity of full-length AR as well as AR variants is to

548 disrupt interactions between AR and specific co-activators and repressors. For instance, Nakka

549 et al. demonstrated that peptides designed to block the interaction between AR and steroid

550 receptor coactivator-1 (SRC-1) inhibited the transcriptional activity of both full-length AR and

551 AR-V7 (Nakka, et al. 2013). Additionally, ectopic expression of SRC-1 increased transcriptional

552 activity of AR-V7, and SRC-1 induced AR-V7 activity was inhibited by expression of FOXO1 due

553 to competition for binding the NTD of AR (Bohrer, et al. 2013). Similarly, treatment of prostate

554 cancer cells with PI3K/AKT inhibitors mediated FOXO1 nuclear translocation leading to a

555 reduction in AR-V7 activity. Moreover, in PTEN-null LNCaP cells, ectopic expression of PTEN

556 reduced full-length and AR-V7 transcriptional activity to a low level similar to that achieved with

557 PI3K/AKT inhibitors (Mediwala, et al. 2013). These studies pinpoint FOXO1 as a potentially

558 important inhibitor of AR activity that is targetable via the AKT pathway, and suggest strategies

559 that could be used in the clinic to combat AR variant driven CRPC (Table 3).

560

561 An additional pathway linked to enzalutamide resistance and AR variant expression is

562 the NF-kB pathway. NFkB2/p52 was shown to enhance AR transcriptional activity as well as

563 boost AR variant expression. For example, overexpression of NFkB/p52 increased AR-V7

564 expression in LNCaP cells, and knockdown of NFkB/p52 expression in 22Rv1 cells decreased

565 mRNA and protein expression of multiple AR variants. In line with this, overexpression of

$566 \mathrm{NFkB} / \mathrm{p} 52$ in LNCaP cells increased survival following enzalutamide treatment and, cells treated

567 chronically with enzalutamide expressed higher endogenous NFkB/p52 than non-treated cells.

568 Importantly, knockdown of either AR variant or NFkB expression increased sensitivity to

569 enzalutamide treatment, suggesting NFkB mediates enzalutamide resistance via induction of

570 AR variants (Nadiminty, et al. 2013). These data suggest that NF-kB inhibition may have a role

571 in the treatment of AR variant driven CRPC (Table 3). Further studies are needed to

572 characterize the expression and role of each of these pathways in enzalutamide and/or 
573 abiraterone acetate resistant metastatic CRPC, and the association of these pathways with AR

574 variant levels.

575

576

Another transcriptional co-factor of AR, Four-and-a-half LIM Domains 2 (FHL2), was

577 shown to co-activate specific AR targets when localized to the nucleus. FLH2 expression

578 increased the transcriptional activity of full-length AR, AR-V7 and the calpain-cleaved AR

579 variant, trAR. In addition, calpain cleavage of the cytoskeletal protein filamin induced nuclear

580 localization of FHL2 leading to increased AR activation (McGrath, et al. 2013). These data

581 provide additional support for the hypothesis that calpain inhibition may have therapeutic benefit

582 in prostate cancer (Table 3). In addition, calpain-mediated cleavage of filamin can affect

583 cytoskeleton organization that leads to increased motility (O'Connell, et al. 2009). Therefore,

584 increases in calpain activity could support metastatic CRPC by reorganizing the cytoskeleton to

585 enhance cell motility and via promoting AR variant synthesis and activation.

586

587 D. Opportunities for Therapeutic Targeting of AR Variants

588 All FDA approved therapeutic agents that target AR exert their action through the AR

589 LBD, and in most cases, AR-dependent resistance emerges after therapy. The discovery of AR

590 variants has highlighted the need for therapies that target AR through alternative mechanisms,

591 such as inhibiting the NTD or by inducing AR degradation. A major question currently is whether

592 AR variant based progression is a predominant mechanism mediating CRPC or if other

593 mechanisms, including bypass of AR dependence and an AR-independent PC (ARIPC), are

594 more common in CRPC progression. ARIPC certainly is known to emerge in CRPC, and may

595 relate to the progression or transformation into neuroendocrine (NEPC) or small cell prostate

596 cancer (Beltran, et al. 2011), or may relate to poorly differentiated CRPC in which AR bypass

597 mechanisms are predominant. Currently, very little is known about ARIPC in the clinic outside

598 of NEPC; however, the CRPC genome is complex, with a number of emerging targets linked to 
599 PI3K, Ras, epigenetics, DNA damage repair and Rb pathways that have AR independent

600 function (Berger et al. 2011). However, for AR-dependent, ligand independent CRPC,

601 strategies to target AR are likely to be successful based on the available evidence to date.

602 However, it will be essential to develop companion diagnostics to determine which CRPC

603 tumors remain AR driven in this setting prior to using novel AR inhibitors.

604

605 Currently, there are several agents being validated pre-clinically for their ability to inhibit

606 AR variants. For example, EPI-001 was discovered in a screen designed to identify compounds

607 capable of binding and inhibiting the AF-1 transactivation region in the AR NTD. The AF-1

608 domain is important for binding many AR co-regulators, and is responsible for the majority of AR

609 transcriptional activity. Binding of EPI-001 to the AR AF-1 region inhibits the ability of AR to

610 interact with proteins such as CREB-binding protein (CBP (Andersen, et al. 2010). Importantly,

611 EPI-001 inhibited transcriptional activity of full-length AR and AR-V12/AR ${ }^{\mathrm{v} 567 e s}$ and decreased

612 tumor growth significantly more than bicalutamide in VCaP xenografts (Myung, et al. 2013).

614 Other AR inhibitors enhance AR degradation as a mechanism to block AR variant

615 activity. For example, ASC-J9 selectively degrades AR protein by disrupting AR dimerization. In

616 addition to reduced AR:AR interaction, ASC-J9 was shown to inhibit AR nuclear localization and

617 promote degradation of both full-length and AR-V7 in 22Rv1 cells (Lai, et al. 2013). In addition

618 ASC-J9 suppressed growth of AR variant positive (22Rv1) and androgen-independent (C81 and

619 C4-2) tumors in castrated mice (Yamashita, et al. 2012). Lin et al. discovered ASC-J9 to be

620 superior to bicalutamide and enzalutamide in both androgen-dependent and -independent

621 prostate cancer cells. Both bicalutamide and enzalutamide enhanced invasion in prostate

622 cancer cell lines through a TGF-B/Smad3/MMP-9 mechanism. In contrast, ASC-J9 treatment

623 did not enhance the invasive capacity of these cells (Lin, et al. 2013). Furthermore, in a co- 
624 culture system with prostate cancer cells and macrophages, bicalutamide and enzalutamide

625 promoted migration of macrophages to prostate cancer cells and resulted in more invasive

626 prostate cancer cells. Importantly, ASC-J9 decreased growth, migration and macrophage

627 infiltration of prostate cancer cells both in vitro and in vivo (Lin et al. 2013). Overall, these third

628 generation AR inhibitors appear to have activity in preclinical models of ADT resistance, but

629 their abilities to block CRPC driven by AR variants requires further evaluation. In addition,

630 siRNA therapeutics directed against the NTD of AR or which promote splice switching toward

631 full-length AR or dominant negative AR45 (Ahrens-Fath, et al. 2005) may be a reasonable

632 strategy in the clinic, provided delivery of siRNA molecules can be targeted in a tumor specific

633 manner (Table 3).

635 Finally, co-targeting strategies, whereby full-length AR is targeted with conventional

636 agents concurrent with targeting of intermediates that may promote AR variant production or

637 nuclear translocation, or which reduce the transcriptional effectors of AR variants, are likely to

638 be more effective clinical strategies (Table 3). For example, given the role of EZH2 and

639 DAB2IP in regulating AR and AR variant activity, EZH2 inhibition with small molecule agents

640 may be an avenue to reduce AR activity without necessarily promoting AR variant production.

641 Inhibitors of cell cycle regulators and proliferation are currently in the clinic in the form of

642 docetaxel and cabazitaxel (a taxane); however, there is concern that the only mechanism of

643 taxane benefit in CRPC is related to the microtubule nuclear transport of AR. If this is the case,

644 it is predicted that CRPC tumors with AR variants lacking the microtubule or dynein binding

645 domain may be resistant to taxanes given their microtubule independent nuclear translocation

646 (Darshan, et al. 2011; Thadani-Mulero, et al. 2012). Ongoing studies such as the TAXYNERGY

647 phase II trial (NCT01718353) should be able to explore whether AR variant expression is linked

648 to resistance to microtubule disruption with docetaxel or cabazitaxel in the clinic. Given the

649 potential cell-cycle specific genes activated by AR variants, cell cycle inhibitors such as PD- 
650 0332991, a CDK4/6 inhibitor (Table 3), may be active in tumors driven by AR variants, and are

651 entering trials in Rb-intact men with metastatic prostate cancer. PI3K, which is involved in the

652 reciprocal regulation of AR activity, and is an inhibitor of the repressive action of FOXO1 on AR

653 activity, may also be a reasonable target strategy when combined with AR inhibitors, and

654 ongoing trials (NCT01385293) will address this combination approach. Finally, inhibition of

655 EMT and epithelial plasticity in tumors driven by AR variants may also be a reasonable broad

656 target for systemic therapy, and strategies in this venue have been recently discussed

657 elsewhere (Bitting et al. 2014).

658

Summary and Future Work

660 In conclusion, AR variant expression appears to emerge in the clinic during CRPC

661 progression and metastatic dissemination. If preclinical models are accurate, the switch from

662 full-length AR to AR variant dominant clones would be selected for during enzalutamide and

663 abiraterone acetate treatment in men with CRPC. However, evidence associating AR variant

664 expression with resistance to enzalutamide and abiraterone acetate treatment is needed from

665 ongoing clinical trials to further support the clinical relevance of AR variants. The association of

666 AR variant expression with other therapies, including docetaxel and cabazitaxel given their

667 putative AR-based transport mechanism, is imperative to study clinically. The anticipated early

668 use of these systemic agents in the clinic may lead to the development of a high proportion of

669 men with either ARIPC or AR variant driven CRPC, which in both cases would represent

670 "androgen-independent" forms of the disease. To address this, several key areas of clinical and

671 translational research will be the development of reliable AR and AR variant biomarkers in

672 CRPC tissue or blood (CTCs or plasma) to measure AR and specific AR variant expression and

673 activity, and the development of therapeutics effective against ARIPC and AR variant DNA

674 binding and transcriptional activation. The clinical relevance of AR variants would undoubtedly

675 be established through documenting clinical benefit with selective AR N-terminal or DNA binding 
676 domain inhibitors after failure of traditional hormonal therapies, and by the pre-treatment

677 identification of loss of C-terminal ligand binding domains in these patients with CRPC.

678 
679 Conflict of Interest: no conflict of interest that could be perceived as prejudicing the impartiality 680 of the research reported

682 Funding: We acknowledge grant support from Veridex postdoctoral training grant (K.E. Ware), 683 National Cancer Institute grant R01 CA127727, (M.A. Garcia-Blanco), Robert B. Goergen 684 Prostate Cancer Foundation Young Investigator Award (A.J. Armstrong), Department of 685 Defense Physician Research Training Award W81XWH-10-1- 0483 (A.J. Armstrong). Studies in 686 the Dehm Lab have been supported by the grants from the National Institutes of Health (R01 687 CA174777 to S.M.D.), an American Cancer Society Research Scholar Grant (RSG-12-031-01, 688 to S.M.D.), and a Department of Defense Prostate Cancer Research Program New Investigator 689 Award (W81XWH-10-1-0353, to S.M.D.). S.M.D. is a Masonic Scholar of the Masonic Cancer 690 Center, University of Minnesota.

691

692 Acknowledgements: The authors thank the studies contributing to Figure 2.

693

694 
696 Ahrens-Fath I, Politz O, Geserick C \& Haendler B 2005 Androgen receptor function is

697 modulated by the tissue-specific AR45 variant. FEBS J 272 74-84.

698 Andersen RJ, Mawji NR, Wang J, Wang G, Haile S, Myung JK, Watt K, Tam T, Yang YC,

699 Banuelos CA, et al. 2010 Regression of castrate-recurrent prostate cancer by a small-molecule

700 inhibitor of the amino-terminus domain of the androgen receptor. Cancer Cell 17 535-546.

701 Armstrong AJ, Marengo MS, Oltean S, Kemeny G, Bitting RL, Turnbull JD, Herold Cl, Marcom

702 PK, George DJ \& Garcia-Blanco MA 2011 Circulating tumor cells from patients with advanced

703 prostate and breast cancer display both epithelial and mesenchymal markers. Mol Cancer Res

$7049997-1007$.

705 Balbas MD, Evans MJ, Hosfield DJ, Wongvipat J, Arora VK, Watson PA, Chen Y, Greene GL,

706 Shen Y \& Sawyers CL 2013 Overcoming mutation-based resistance to antiandrogens with

707 rational drug design. Elife 2 e00499.

708 Beltran H, Rickman DS, Park K, Chae SS, Sboner A, MacDonald TY, Wang Y, Sheikh KL, Terry

709 S, Tagawa ST, et al. 2011 Molecular characterization of neuroendocrine prostate cancer and

710 identification of new drug targets. Cancer Discov 1 487-495.

711 Berger MF, Lawrence MS, Demichelis F, Drier Y, Cibulskis K, Sivachenko AY, Sboner A,

712 Esgueva R, Pflueger D, Sougnez C, et al. 2011 The genomic complexity of primary human

713 prostate cancer. Nature $\mathbf{4 7 0}$ 214-220.

714 Bergerat JP \& Ceraline J 2009 Pleiotropic functional properties of androgen receptor mutants in

715 prostate cancer. Hum Mutat 30 145-157.

716 Bitting RL, Schaeffer D, Somarelli JA, Garcia-Blanco MA \& Armstrong AJ 2014 The Role of

717 Epithelial Plasticity in Prostate Cancer Dissemination and Treatment Resistance. Cancer

718 Metastasis Reviews In Press. 
719 Bohrer LR, Liu P, Zhong J, Pan Y, Angstman J, Brand LJ, Dehm SM \& Huang H 2013 FOXO1

720 binds to the TAU5 motif and inhibits constitutively active androgen receptor splice variants.

721 Prostate 73 1017-1027.

722 Cai C, He HH, Chen S, Coleman I, Wang H, Fang Z, Chen S, Nelson PS, Liu XS, Brown M, et

723 al. 2011 Androgen receptor gene expression in prostate cancer is directly suppressed by the

724 androgen receptor through recruitment of lysine-specific demethylase 1. Cancer Cell 20 457-

725471.

726 Ceraline J, Erdmann E, Erbs P, Deslandres-Cruchant M, Jacqmin D, Duclos B, Klein-Soyer C,

727 Dufour P \& Bergerat JP 2003 A yeast-based functional assay for the detection of the mutant

728 androgen receptor in prostate cancer. Eur J Endocrinol 148 99-110.

729 Chan SC, Li Y \& Dehm SM 2012 Androgen receptor splice variants activate androgen receptor

730 target genes and support aberrant prostate cancer cell growth independent of canonical

731 androgen receptor nuclear localization signal. J Biol Chem 287 19736-19749.

732 Chang KH, Li R, Kuri B, Lotan Y, Roehrborn CG, Liu J, Vessella R, Nelson PS, Kapur P, Guo X,

733 et al. 2013 A Gain-of-Function Mutation in DHT Synthesis in Castration-Resistant Prostate

734 Cancer. Cell 154 1074-1084.

735 Chen CD, Welsbie DS, Tran C, Baek SH, Chen R, Vessella R, Rosenfeld MG \& Sawyers CL

7362004 Molecular determinants of resistance to antiandrogen therapy. Nat Med 10 33-39.

737 Chen H, Pong RC, Wang Z \& Hsieh JT 2002 Differential regulation of the human gene DAB2IP

738 in normal and malignant prostatic epithelia: cloning and characterization. Genomics 79 573-581.

739 Chen Y, Chi P, Rockowitz S, laquinta PJ, Shamu T, Shukla S, Gao D, Sirota I, Carver BS,

740 Wongvipat J, et al. 2013 ETS factors reprogram the androgen receptor cistrome and prime

741 prostate tumorigenesis in response to PTEN loss. Nat Med 19 1023-1029.

742 Choi N, Zhang B, Zhang L, Ittmann M \& Xin L 2012 Adult murine prostate basal and luminal

743 cells are self-sustained lineages that can both serve as targets for prostate cancer initiation.

744 Cancer Cell 21 253-265. 
745 Clegg NJ, Wongvipat J, Joseph JD, Tran C, Ouk S, Dilhas A, Chen Y, Grillot K, Bischoff ED, Cai

746 L, et al. 2012 ARN-509: a novel antiandrogen for prostate cancer treatment. Cancer Res 72

$747 \quad 1494-1503$.

748 Coffey K \& Robson CN 2012 Regulation of the androgen receptor by post-translational

749 modifications. J Endocrinol 215 221-237.

750 Cottard F, Asmane I, Erdmann E, Bergerat JP, Kurtz JE \& Ceraline J 2013 Constitutively active

751 androgen receptor variants upregulate expression of mesenchymal markers in prostate cancer

752 cells. PLoS One 8 e63466.

753 Darshan MS, Loftus MS, Thadani-Mulero M, Levy BP, Escuin D, Zhou XK, Gjyrezi A, Chanel-

754 Vos C, Shen R, Tagawa ST, et al. 2011 Taxane-induced blockade to nuclear accumulation of

755 the androgen receptor predicts clinical responses in metastatic prostate cancer. Cancer Res 71

$756 \quad 6019-6029$.

757 de Bono JS, Logothetis CJ, Molina A, Fizazi K, North S, Chu L, Chi KN, Jones RJ, Goodman

758 OB, Jr., Saad F, et al. 2011 Abiraterone and increased survival in metastatic prostate cancer. $N$

759 Engl J Med 364 1995-2005.

760 Dehm SM, Schmidt LJ, Heemers HV, Vessella RL \& Tindall DJ 2008 Splicing of a novel

761 androgen receptor exon generates a constitutively active androgen receptor that mediates

762 prostate cancer therapy resistance. Cancer Res 68 5469-5477.

763 Freedland SJ, Humphreys EB, Mangold LA, Eisenberger M, Dorey FJ, Walsh PC \& Partin AW

7642007 Death in patients with recurrent prostate cancer after radical prostatectomy: prostate-

765 specific antigen doubling time subgroups and their associated contributions to all-cause

766 mortality. J Clin Oncol 25 1765-1771.

767 Garraway LA \& Sellers WR 2006 Lineage dependency and lineage-survival oncogenes in

768 human cancer. Nat Rev Cancer 6 593-602. 
769 Ghigna C, Giordano S, Shen H, Benvenuto F, Castiglioni F, Comoglio PM, Green MR, Riva S \&

770 Biamonti G 2005 Cell motility is controlled by SF2/ASF through alternative splicing of the Ron

771 protooncogene. Mol Cell 20 881-890.

772 Gillis JL, Selth LA, Centenera MM, Townley SL, Sun S, Plymate SR, Tilley WD \& Butler LM

7732013 Constitutively-active androgen receptor variants function independently of the HSP90

774 chaperone but do not confer resistance to HSP90 inhibitors. Oncotarget 4 691-704.

775 Goldstein AS, Huang J, Guo C, Garraway IP \& Witte ON 2010 Identification of a cell of origin for

776 human prostate cancer. Science 329 568-571.

777 Gu W, Zhang F \& Lupski JR 2008 Mechanisms for human genomic rearrangements.

778 Pathogenetics 14.

779 Guo Z, Yang X, Sun F, Jiang R, Linn DE, Chen H, Kong X, Melamed J, Tepper CG, Kung HJ, et

780 al. 2009 A novel androgen receptor splice variant is up-regulated during prostate cancer

781 progression and promotes androgen depletion-resistant growth. Cancer Res 69 2305-2313.

782 Gurusamy D, Gray JK, Pathrose P, Kulkarni RM, Finkleman FD \& Waltz SE 2013 Myeloid-

783 specific expression of Ron receptor kinase promotes prostate tumor growth. Cancer Res 73

$784 \quad 1752-1763$.

785 Haffner MC, Aryee MJ, Toubaji A, Esopi DM, Albadine R, Gurel B, Isaacs WB, Bova GS, Liu W,

$786 \mathrm{Xu} \mathrm{J}$, et al. 2010 Androgen-induced TOP2B-mediated double-strand breaks and prostate cancer

787 gene rearrangements. Nat Genet 42 668-675.

788 Han G, Foster BA, Mistry S, Buchanan G, Harris JM, Tilley WD \& Greenberg NM 2001

789 Hormone status selects for spontaneous somatic androgen receptor variants that demonstrate

790 specific ligand and cofactor dependent activities in autochthonous prostate cancer. J Biol Chem

791276 11204-11213.

792 Hastings PJ, Ira G \& Lupski JR 2009 A microhomology-mediated break-induced replication

793 model for the origin of human copy number variation. PLoS Genet 5 e1000327. 
794 Hornberg E, Ylitalo EB, Crnalic S, Antti H, Stattin P, Widmark A, Bergh A \& Wikstrom P 2011

795 Expression of androgen receptor splice variants in prostate cancer bone metastases is

796 associated with castration-resistance and short survival. PLoS One 6 e19059.

797 Hu R, Dunn TA, Wei S, Isharwal S, Veltri RW, Humphreys E, Han M, Partin AW, Vessella RL,

798 Isaacs WB, et al. 2009 Ligand-independent androgen receptor variants derived from splicing of

799 cryptic exons signify hormone-refractory prostate cancer. Cancer Res 69 16-22.

$800 \mathrm{Hu}$ R, Isaacs WB \& Luo J 2011 A snapshot of the expression signature of androgen receptor

801 splicing variants and their distinctive transcriptional activities. Prostate 71 1656-1667.

802 Hu R, Lu C, Mostaghel EA, Yegnasubramanian S, Gurel M, Tannahill C, Edwards J, Isaacs WB,

803 Nelson PS, Bluemn E, et al. 2012 Distinct transcriptional programs mediated by the ligand-

804 dependent full-length androgen receptor and its splice variants in castration-resistant prostate

805 cancer. Cancer Res 72 3457-3462.

806 Hussain M, Tangen CM, Higano C, Schelhammer PF, Faulkner J, Crawford ED, Wilding G,

807 Akdas A, Small EJ, Donnelly B, et al. 2006 Absolute prostate-specific antigen value after

808 androgen deprivation is a strong independent predictor of survival in new metastatic prostate

809 cancer: data from Southwest Oncology Group Trial 9346 (INT-0162). J Clin Oncol 24 3984-

8103990.

811 Joseph JD, Lu N, Qian J, Sensintaffar J, Shao G, Brigham D, Moon M, Maneval EC, Chen I,

812 Darimont B, et al. 2013 A Clinically Relevant Androgen Receptor Mutation Confers Resistance

813 to Second-Generation Antiandrogens Enzalutamide and ARN-509. Cancer Discov 3 1020-1029.

814 Joung JK \& Sander JD 2013 TALENs: a widely applicable technology for targeted genome

815 editing. Nat Rev Mol Cell Biol 14 49-55.

816 Korpal M, Korn JM, Gao X, Rakiec DP, Ruddy DA, Doshi S, Yuan J, Kovats SG, Kim S, Cooke

817 VG, et al. 2013 An F876L Mutation in Androgen Receptor Confers Genetic and Phenotypic

818 Resistance to MDV3100 (Enzalutamide). Cancer Discov 3 1030-1043. 
819 Koumbaris G, Hatzisevastou-Loukidou H, Alexandrou A, loannides M, Christodoulou C,

820 Fitzgerald T, Rajan D, Clayton S, Kitsiou-Tzeli S, Vermeesch JR, et al. 2011 FoSTeS, MMBIR

821 and NAHR at the human proximal Xp region and the mechanisms of human Xq isochromosome 822 formation. Hum Mol Genet 20 1925-1936.

823 Lai KP, Huang CK, Chang YJ, Chung CY, Yamashita S, Li L, Lee SO, Yeh S \& Chang C 2013

824 New therapeutic approach to suppress castration-resistant prostate cancer using ASC-J9 via

825 targeting androgen receptor in selective prostate cells. Am J Pathol 182 460-473.

826 Li Y, Alsagabi M, Fan D, Bova GS, Tewfik AH \& Dehm SM 2011 Intragenic rearrangement and

827 altered RNA splicing of the androgen receptor in a cell-based model of prostate cancer

828 progression. Cancer Res 71 2108-2117.

829 Li Y, Chan SC, Brand LJ, Hwang TH, Silverstein KA \& Dehm SM 2013 Androgen receptor splice

830 variants mediate enzalutamide resistance in castration-resistant prostate cancer cell lines.

831 Cancer Res 73 483-489.

832 Li Y, Hwang TH, Oseth LA, Hauge A, Vessella RL, Schmechel SC, Hirsch B, Beckman KB,

833 Silverstein KA \& Dehm SM 2012 AR intragenic deletions linked to androgen receptor splice

834 variant expression and activity in models of prostate cancer progression. Oncogene 31 4759-

8354767.

836 Libertini SJ, Tepper CG, Rodriguez V, Asmuth DM, Kung HJ \& Mudryj M 2007 Evidence for

837 calpain-mediated androgen receptor cleavage as a mechanism for androgen independence.

838 Cancer Res 67 9001-9005.

839 Lin C, Yang L, Tanasa B, Hutt K, Ju BG, Ohgi K, Zhang J, Rose DW, Fu XD, Glass CK, et al.

8402009 Nuclear receptor-induced chromosomal proximity and DNA breaks underlie specific

841 translocations in cancer. Cell 139 1069-1083.

842 Lin TH, Izumi K, Lee SO, Lin WJ, Yeh S \& Chang C 2013 Anti-androgen receptor ASC-J9

843 versus anti-androgens MDV3100 (Enzalutamide) or Casodex (Bicalutamide) leads to opposite 
844 effects on prostate cancer metastasis via differential modulation of macrophage infiltration and 845 STAT3-CCL2 signaling. Cell Death Dis 4 e764.

846 Liu G, Sprenger C, Sun S, Epilepsia KS, Haugk K, Zhang X, Coleman I, Nelson PS \& Plymate S 847 2013a AR Variant AR(v567es) Induces Carcinogenesis in a Novel Transgenic Mouse Model of 848 Prostate Cancer. Neoplasia 15 1009-1017.

849 Liu LL, Xie N, Sun S, Plymate S, Mostaghel E \& Dong X 2013b Mechanisms of the androgen 850 receptor splicing in prostate cancer cells. Oncogene.

851 Loriot Y, Bianchini D, Ileana E, Sandhu S, Patrikidou A, Pezaro C, Albiges L, Attard G, Fizazi K,

852 De Bono JS, et al. 2013 Antitumour activity of abiraterone acetate against metastatic castration853 resistant prostate cancer progressing after docetaxel and enzalutamide (MDV3100). Ann Oncol 85424 1807-1812.

855 Mani RS, Tomlins SA, Callahan K, Ghosh A, Nyati MK, Varambally S, Palanisamy N \&

856 Chinnaiyan AM 2009 Induced chromosomal proximity and gene fusions in prostate cancer.

857 Science 3261230.

858 McGrath MJ, Binge LC, Sriratana A, Wang H, Robinson PA, Pook D, Fedele CG, Brown S, 859 Dyson JM, Cottle DL, et al. 2013 Regulation of the Transcriptional Coactivator FHL2 Licenses

860 Activation of the Androgen Receptor in Castrate-Resistant Prostate Cancer. Cancer Res 73 $861 \quad 5066-5079$.

862 Mediwala SN, Sun H, Szafran AT, Hartig SM, Sonpavde G, Hayes TG, Thiagarajan P, Mancini 863 MA \& Marcelli M 2013 The activity of the androgen receptor variant AR-V7 is regulated by 864 FOXO1 in a PTEN-PI3K-AKT-dependent way. Prostate 73 267-277.

865 Min J, Zaslavsky A, Fedele G, McLaughlin SK, Reczek EE, De Raedt T, Guney I, Strochlic DE, 866 Macconaill LE, Beroukhim R, et al. 2010 An oncogene-tumor suppressor cascade drives 867 metastatic prostate cancer by coordinately activating Ras and nuclear factor-kappaB. Nat Med $86816286-294$. 
869 Mostaghel EA, Marck BT, Plymate SR, Vessella RL, Balk S, Matsumoto AM, Nelson PS \&

870 Montgomery RB 2011 Resistance to CYP17A1 inhibition with abiraterone in castration-resistant

871 prostate cancer: induction of steroidogenesis and androgen receptor splice variants. Clin

872 Cancer Res 17 5913-5925.

873 Mulholland DJ, Kobayashi N, Ruscetti M, Zhi A, Tran LM, Huang J, Gleave M \& Wu H 2012

874 Pten loss and RAS/MAPK activation cooperate to promote EMT and metastasis initiated from

875 prostate cancer stem/progenitor cells. Cancer Res 72 1878-1889.

876 Myung JK, Banuelos CA, Fernandez JG, Mawji NR, Wang J, Tien AH, Yang YC, Tavakoli I,

877 Haile S, Watt K, et al. 2013 An androgen receptor N-terminal domain antagonist for treating

878 prostate cancer. J Clin Invest 123 2948-2960.

879 Nadiminty N, Tummala R, Liu C, Yang J, Lou W, Evans CP \& Gao AC 2013 NF-kappaB2/p52

880 induces resistance to enzalutamide in prostate cancer: role of androgen receptor and its

881 variants. Mol Cancer Ther 12 1629-1637.

882 Nakka M, Agoulnik IU \& Weigel NL 2013 Targeted disruption of the p160 coactivator interface of

883 androgen receptor (AR) selectively inhibits AR activity in both androgen-dependent and

884 castration-resistant AR-expressing prostate cancer cells. Int J Biochem Cell Biol 45 763-772.

885 Noonan KL, North S, Bitting RL, Armstrong AJ, Ellard SL \& Chi KN 2013 Clinical activity of

886 abiraterone acetate in patients with metastatic castration-resistant prostate cancer progressing

887 after enzalutamide. Ann Oncol 24 1802-1807.

888 Nyquist MD, Li Y, Hwang TH, Manlove LS, Vessella RL, Silverstein KA, Voytas DF \& Dehm SM

8892013 TALEN-engineered AR gene rearrangements reveal endocrine uncoupling of androgen

890 receptor in prostate cancer. Proc Natl Acad Sci U S A.

891 O'Connell MP, Fiori JL, Baugher KM, Indig FE, French AD, Camilli TC, Frank BP, Earley R,

892 Hoek KS, Hasskamp JH, et al. 2009 Wnt5A activates the calpain-mediated cleavage of filamin

893 A. J Invest Dermatol 129 1782-1789. 
894 Oh SJ, Erb HH, Hobisch A, Santer FR \& Culig Z 2012 Sorafenib decreases proliferation and 895 induces apoptosis of prostate cancer cells by inhibition of the androgen receptor and Akt 896 signaling pathways. Endocr Relat Cancer 19 305-319.

897 Rios-Doria J, Kuefer R, Ethier SP \& Day ML 2004 Cleavage of beta-catenin by calpain in 898 prostate and mammary tumor cells. Cancer Res 64 7237-7240.

899 Ryan CJ, Smith MR, de Bono JS, Molina A, Logothetis CJ, de Souza P, Fizazi K, Mainwaring P,

900 Piulats JM, Ng S, et al. 2013 Abiraterone in metastatic prostate cancer without previous

901 chemotherapy. N Engl J Med 368 138-148.

902 Schaeffer AJ 2013 Editorial comment. Urology 81 555-556; discussion 556.

903 Scher HI, Beer TM, Higano CS, Anand A, Taplin ME, Efstathiou E, Rathkopf D, Shelkey J, Yu

904 EY, Alumkal J, et al. 2010 Antitumour activity of MDV3100 in castration-resistant prostate

905 cancer: a phase 1-2 study. Lancet 375 1437-1446.

906 Scher HI, Fizazi K, Saad F, Taplin ME, Sternberg CN, Miller K, de Wit R, Mulders P, Chi KN,

907 Shore ND, et al. 2012 Increased survival with enzalutamide in prostate cancer after

908 chemotherapy. N Engl J Med 367 1187-1197.

909 Schiewer MJ, Goodwin JF, Han S, Brenner JC, Augello MA, Dean JL, Liu F, Planck JL,

910 Ravindranathan P, Chinnaiyan AM, et al. 2012 Dual roles of PARP-1 promote cancer growth

911 and progression. Cancer Discov 2 1134-1149.

912 Shafi AA, Cox MB \& Weigel NL 2013 Androgen receptor splice variants are resistant to

913 inhibitors of Hsp90 and FKBP52, which alter androgen receptor activity and expression.

914 Steroids 78 548-554.

915 Sharma A, Yeow WS, Ertel A, Coleman I, Clegg N, Thangavel C, Morrissey C, Zhang X,

916 Comstock CE, Witkiewicz AK, et al. 2010 The retinoblastoma tumor suppressor controls

917 androgen signaling and human prostate cancer progression. J Clin Invest 120 4478-4492. 
918 Shiota M, Yokomizo A, Fujimoto N \& Naito S 2011 Androgen receptor cofactors in prostate

919 cancer: potential therapeutic targets of castration-resistant prostate cancer. Curr Cancer Drug

920 Targets $11870-881$.

921 Siegel RD 2013 American Society of Clinical Oncology Quality Care Symposium 2012: re-

922 engineering your practice to deliver quality and value. J Oncol Pract 9 160-161.

923 Stanbrough M, Bubley GJ, Ross K, Golub TR, Rubin MA, Penning TM, Febbo PG \& Balk SP

9242006 Increased expression of genes converting adrenal androgens to testosterone in androgen-

925 independent prostate cancer. Cancer Res 66 2815-2825.

926 Sun F, Chen HG, Li W, Yang X, Wang X, Jiang R, Guo Z, Chen H, Huang J, Borowsky AD, et

927 al. 2013 Androgen receptor splice variant AR3 promotes prostate cancer via modulating

928 expression of autocrine/paracrine factors. J Biol Chem.

929 Sun S, Sprenger CC, Vessella RL, Haugk K, Soriano K, Mostaghel EA, Page ST, Coleman IM,

930 Nguyen HM, Sun H, et al. 2010 Castration resistance in human prostate cancer is conferred by

931 a frequently occurring androgen receptor splice variant. J Clin Invest 120 2715-2730.

932 Sun Y, Wang BE, Leong KG, Yue P, Li L, Jhunjhunwala S, Chen D, Seo K, Modrusan Z, Gao

933 WQ, et al. 2012 Androgen deprivation causes epithelial-mesenchymal transition in the prostate:

934 implications for androgen-deprivation therapy. Cancer Res 72 527-536.

935 Tanaka H, Kono E, Tran CP, Miyazaki H, Yamashiro J, Shimomura T, Fazli L, Wada R, Huang

$936 \mathrm{~J}$, Vessella RL, et al. 2010 Monoclonal antibody targeting of N-cadherin inhibits prostate cancer

937 growth, metastasis and castration resistance. Nat Med 16 1414-1420.

938 Taylor BS, Schultz N, Hieronymus H, Gopalan A, Xiao Y, Carver BS, Arora VK, Kaushik P,

939 Cerami E, Reva B, et al. 2010 Integrative genomic profiling of human prostate cancer. Cancer

$940 \quad$ Cell 18 11-22.

941 Tepper CG, Boucher DL, Ryan PE, Ma AH, Xia L, Lee LF, Pretlow TG \& Kung HJ 2002

942 Characterization of a novel androgen receptor mutation in a relapsed CWR22 prostate cancer

943 xenograft and cell line. Cancer Res 62 6606-6614. 
944 Thadani-Mulero M, Nanus DM \& Giannakakou P 2012 Androgen receptor on the move:

945 boarding the microtubule expressway to the nucleus. Cancer Res 72 4611-4615.

946 Tran C, Ouk S, Clegg NJ, Chen Y, Watson PA, Arora V, Wongvipat J, Smith-Jones PM, Yoo D,

947 Kwon A, et al. 2009 Development of a second-generation antiandrogen for treatment of

948 advanced prostate cancer. Science 324 787-790.

949 Tsai HC, Boucher DL, Martinez A, Tepper CG \& Kung HJ 2012 Modeling truncated AR

950 expression in a natural androgen responsive environment and identification of RHOB as a direct

951 transcriptional target. PLoS One 7 e49887.

952 van de Wijngaart DJ, Dubbink HJ, van Royen ME, Trapman J \& Jenster G 2012 Androgen

953 receptor coregulators: recruitment via the coactivator binding groove. Mol Cell Endocrinol 352

$954 \quad 57-69$.

955 Wang X, Kruithof-de Julio M, Economides KD, Walker D, Yu H, Halili MV, Hu YP, Price SM,

956 Abate-Shen C \& Shen MM 2009 A luminal epithelial stem cell that is a cell of origin for prostate

957 cancer. Nature $\mathbf{4 6 1} 495-500$.

958 Wang ZA, Mitrofanova A, Bergren SK, Abate-Shen C, Cardiff RD, Califano A \& Shen MM 2013

959 Lineage analysis of basal epithelial cells reveals their unexpected plasticity and supports a cell-

960 of-origin model for prostate cancer heterogeneity. Nat Cell Biol 15 274-283.

961 Watson PA, Chen YF, Balbas MD, Wongvipat J, Socci ND, Viale A, Kim K \& Sawyers CL 2010

962 Constitutively active androgen receptor splice variants expressed in castration-resistant prostate

963 cancer require full-length androgen receptor. Proc Natl Acad Sci U S A 107 16759-16765.

964 Weischenfeldt J, Simon R, Feuerbach L, Schlangen K, Weichenhan D, Minner S, Wuttig D,

965 Warnatz HJ, Stehr H, Rausch T, et al. 2013 Integrative genomic analyses reveal an androgen-

966 driven somatic alteration landscape in early-onset prostate cancer. Cancer Cell 23 159-170.

967 Wu K, Liu J, Tseng SF, Gore C, Ning Z, Sharifi N, Fazli L, Gleave M, Kapur P, Xiao G, et al.

9682013 The role of DAB2IP in androgen receptor activation during prostate cancer progression.

969 Oncogene. 
970 Xu K, Wu ZJ, Groner AC, He HH, Cai C, Lis RT, Wu X, Stack EC, Loda M, Liu T, et al. 2012

971 EZH2 oncogenic activity in castration-resistant prostate cancer cells is Polycomb-independent.

972 Science 338 1465-1469.

973 Yamashita S, Lai KP, Chuang KL, Xu D, Miyamoto H, Tochigi T, Pang ST, Li L, Arai Y, Kung

$974 \mathrm{HJ}$, et al. 2012 ASC-J9 suppresses castration-resistant prostate cancer growth through

975 degradation of full-length and splice variant androgen receptors. Neoplasia 14 74-83.

976 Yang X, Guo Z, Sun F, Li W, Alfano A, Shimelis H, Chen M, Brodie AM, Chen H, Xiao Z, et al.

9772011 Novel membrane-associated androgen receptor splice variant potentiates proliferative and

978 survival responses in prostate cancer cells. J Biol Chem 286 36152-36160.

979 Zengerling F, Streicher W, Schrader AJ, Schrader M, Nitzsche B, Cronauer MV \& Hopfner M

9802012 Effects of sorafenib on C-terminally truncated androgen receptor variants in human

981 prostate cancer cells. Int J Mol Sci 13 11530-11542.

982 Zhang X, Morrissey C, Sun S, Ketchandji M, Nelson PS, True LD, Vakar-Lopez F, Vessella RL

983 \& Plymate SR 2011 Androgen receptor variants occur frequently in castration resistant prostate

984 cancer metastases. PLoS One 6 e27970.

985 Zhao H, Coram MA, Nolley R, Reese SW, Young SR \& Peehl DM 2012 Transcript levels of

986 androgen receptor variant AR-V1 or AR-V7 do not predict recurrence in patients with prostate

987 cancer at indeterminate risk for progression. J Urol 188 2158-2164.

988 
991 Figure 1. AR gene rearrangements and AR variants identified in prostate cancer. A. Schematic

992 of AR gene mutations and genomic alterations with cryptic exons indicated in red. B. Schematic 993 of AR and variant mRNA. Functional domains represented as $\mathrm{NH}$-terminal domain (NTD) in 994 blue, DNA binding domain (DBD) in green, hinge region in purple and ligand binding domain

995 (LBD) in orange. Cryptic exons are colored red. Solid regions represent the open reading frame.

996 Star indicates calpain recognition site for proteolytic cleavage. Distinct AR variants AR-V8, AR-

997 V10 and AR-V11 are represented by one schematic.

999 Figure 2. A-D. Clinical associations of AR variant expression. A. Zhang et al. (Zhang et al. 2011)

1000 probed for AR protein levels using two AR-specific antibodies directed against the N- and C-

1001 termini. This allowed for the inference of AR splice variant expression in in these tissues,

1002 indicating that metastatic CRPC tissue contains a higher prevalence of $\mathrm{N}+\mathrm{C}$ - or $\mathrm{N}+\mathrm{C}$-reduced

1003 expression patterns as compared to localized disease (Zhang et al. 2011). B. Using these same

1004 antibodies, Zhang et al. (Zhang et al. 2011) showed that PSA production by tumor cells appears

1005 to be reduced in some N+C-reduced tumors. C. Hornberg et al. (Hornberg et al. 2011)

1006 demonstrated by Kaplan-Meier analysis that high expression of AR -V7 in bone metastatic

1007 CRPC at surgery is associated with death from prostate cancer. D. Guo et al. (Guo et al. 2009)

1008 demonstrated that survival free of PSA recurrence was associated with expression of AR-V7

1009 (AR3) in localized prostate cancer. Reprinted by permission from the American Association for

1010 Cancer Research: Guo Z et al., A novel androgen receptor splice variant is up-regulated during

1011 prostate cancer progression and promotes androgen depletion-resistant growth. Cancer

1012 Research, March 15, 2009, 69(6), 2305-2313, doi:10.1158/0008-5472.

1013 


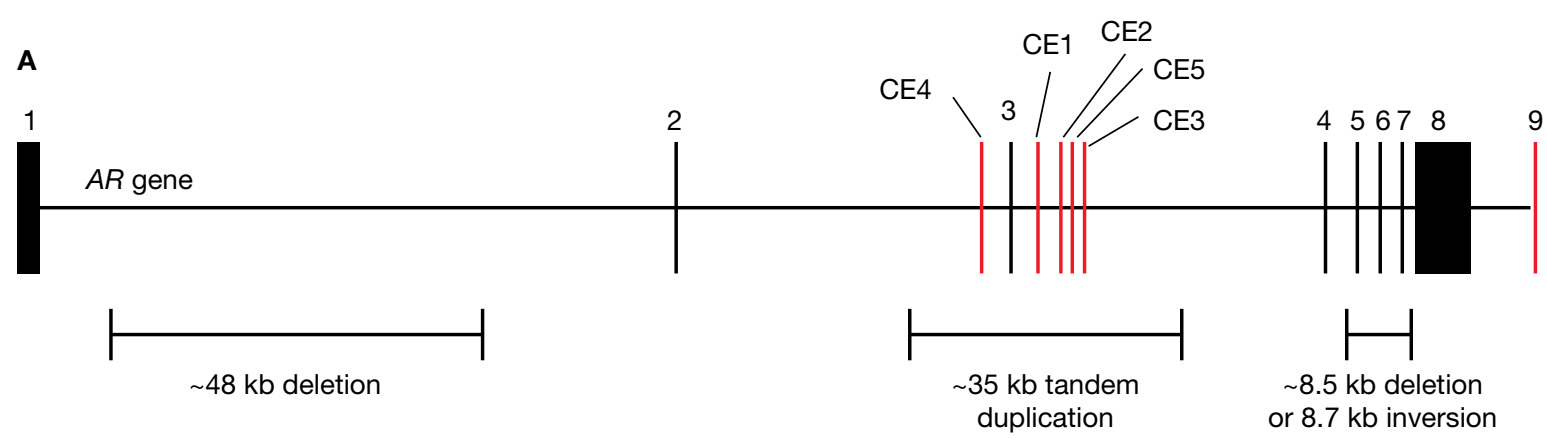

B

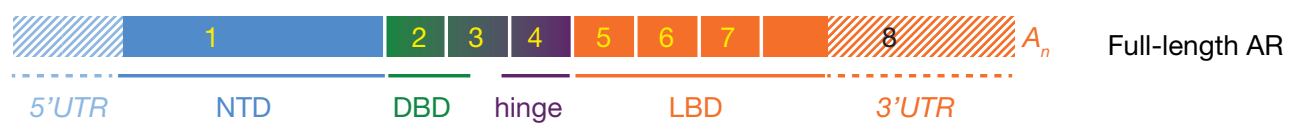

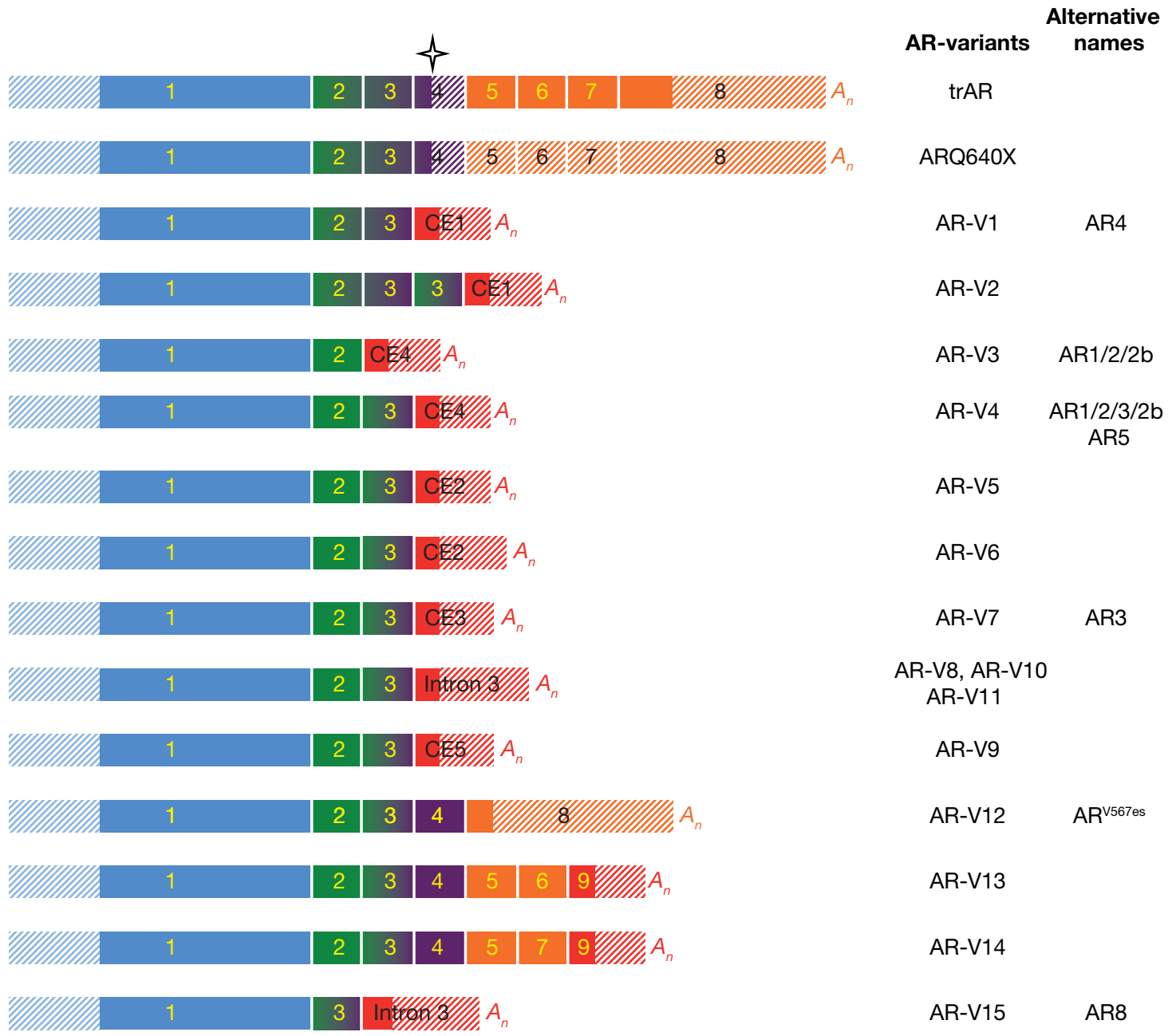


A

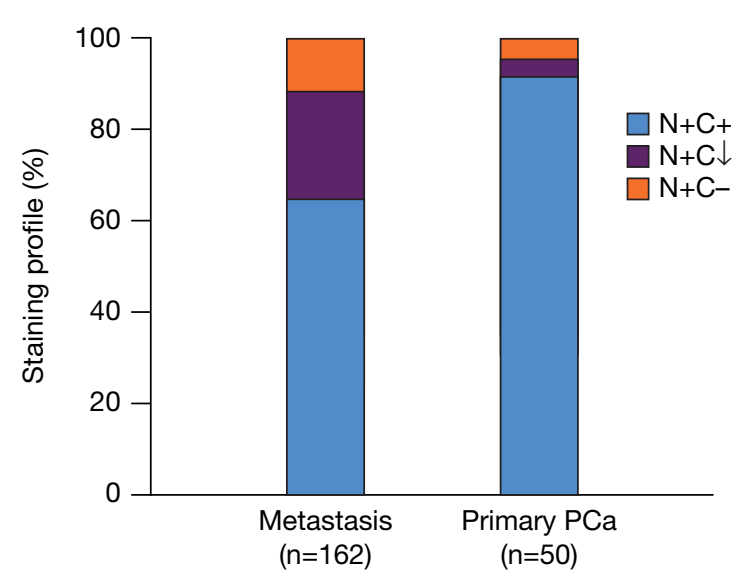

C

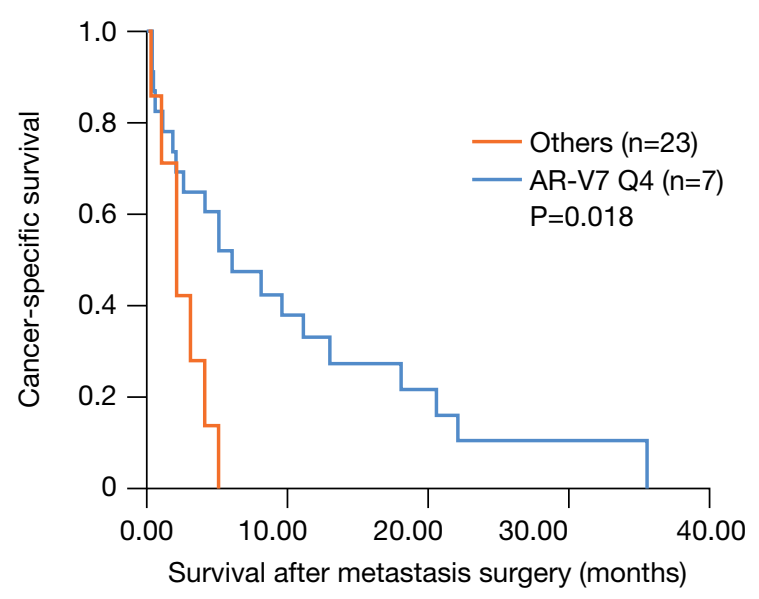

B

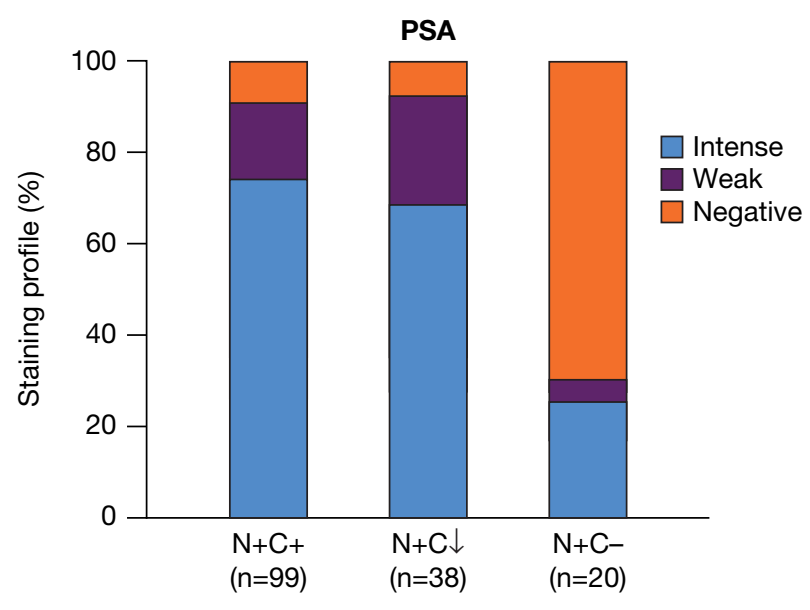

D

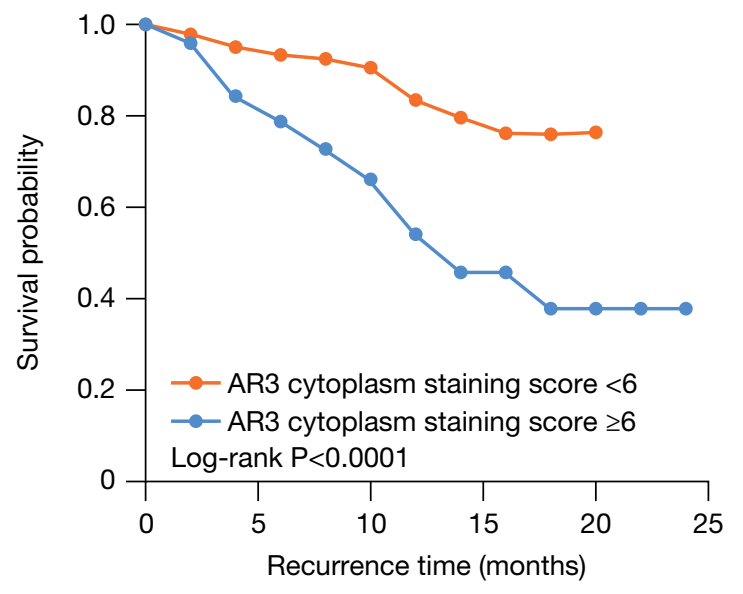


Table 1. Characteristics and expression differences of AR variants.

\begin{tabular}{|c|c|c|c|c|c|c|}
\hline \multirow[b]{2}{*}{$\begin{array}{c}\text { AR } \\
\text { Variant }\end{array}$} & \multirow{2}{*}{$\begin{array}{l}\text { Splice } \\
\text { Junction }\end{array}$} & \multirow[b]{2}{*}{ Activity } & \multicolumn{2}{|c|}{ Expression } & \multirow[b]{2}{*}{$A R$ Genomic alterations } & \multirow[b]{2}{*}{ Ref. } \\
\hline & & & $\begin{array}{l}\text { Human CRPC } \\
\text { tissue }\end{array}$ & Cell Line & & \\
\hline $\operatorname{trAR}$ & 648 a.a. & constitutive & & 22Rv1 & $\begin{array}{l}35 \mathrm{~kb} \text { tandem duplication } \\
\text { (exon } 3 \text { ) }\end{array}$ & (Libertini et al. 2007) \\
\hline ARQ640X & $1 / 2 / 3 / 4$ & constitutive & mRNA & & point mutation & (Ceraline et al. 2003) \\
\hline AR-V1 & 3/CE1 & conditional & mRNA & VCaP & Amplification & $\begin{array}{l}\text { (Guo et al. 2009; Hu et } \\
\text { al. 2009) }\end{array}$ \\
\hline AR-V2 & 3/3/CE1 & NA & & 22Rv1 & $\begin{array}{l}35 \mathrm{~kb} \text { tandem duplication } \\
\text { (exon } 3 \text { ) }\end{array}$ & $\begin{array}{l}\text { (Guo et al. 2009; Hu et } \\
\text { al. 2009) }\end{array}$ \\
\hline AR-V3 & 2/CE4 & constitutive & & 22Rv1 & $\begin{array}{l}35 \mathrm{~kb} \text { tandem duplication } \\
\text { (exon 3) }\end{array}$ & $\begin{array}{l}\text { (Dehm et al. 2008; Hu et } \\
\text { al. 2009) }\end{array}$ \\
\hline AR-V4 & 3/CE4 & constitutive & & 22Rv1 & $\begin{array}{l}35 \mathrm{~kb} \text { tandem duplication } \\
\text { (exon 3) }\end{array}$ & $\begin{array}{l}\text { (Dehm et al. 2008; Guo } \\
\text { et al. 2009; Hu et al. } \\
\text { 2009) }\end{array}$ \\
\hline AR-V5 & 3/CE2 & NA & mRNA & 22Rv1 & $\begin{array}{l}35 \mathrm{~kb} \text { tandem duplication } \\
\text { (exon 3) }\end{array}$ & \multirow[t]{2}{*}{ (Hu et al. 2009) } \\
\hline \multirow[t]{2}{*}{ AR-V6 } & $3 / C E 2$ & NA & mRNA & 22Rv1 & $\begin{array}{l}35 \mathrm{~kb} \text { tandem duplication } \\
\text { (exon 3) }\end{array}$ & \\
\hline & & & & LNCAP95 & & \\
\hline \multirow[t]{3}{*}{ AR-V7 } & \multirow[t]{3}{*}{$3 /$ CE3 } & \multirow[t]{3}{*}{ constitutive } & \multirow{3}{*}{ mRNA, protein } & VCaP & Amplification & \multirow{3}{*}{$\begin{array}{l}\text { (Guo et al. 2009; Hu et } \\
\text { al. 2009) }\end{array}$} \\
\hline & & & & 22Rv1 & $\begin{array}{l}35 \mathrm{~kb} \text { tandem duplication } \\
\text { (exon } 3 \text { ) }\end{array}$ & \\
\hline & & & & CWR-R1 & $48 \mathrm{~kb}$ deletion (intron 1 ) & \\
\hline AR-V8 & 3/intron 3 & NA & & VCaP & Amplification & (Watson et al. 2010) \\
\hline AR-V9 & 3/CE5 & conditional & mRNA & VCaP & Amplification & $\begin{array}{l}\text { (Hu et al. 2011; Watson } \\
\text { et al. 2010) }\end{array}$ \\
\hline AR-V10 & 3/intron 3 & NA & & VCaP & Amplification & (Watson et al. 2010) \\
\hline \multirow[t]{2}{*}{ AR-V11 } & 3/intron 3 & NA & & VCaP & Amplification & (Watson et al. 2010) \\
\hline & & & & LuCaP 86.2 & $8.5 \mathrm{~kb}$ deletion (exon $5,6,7$ ) & \\
\hline \multirow{3}{*}{$\begin{array}{l}\text { AR } \\
\text { v567es }\end{array}$} & \multirow{3}{*}{4,8} & \multirow{3}{*}{ constitutive } & \multirow{3}{*}{ mRNA } & LuCaP 136 & 8.7kb inversion (exon $5,6,7)$ & \multirow{3}{*}{$\begin{array}{l}\text { (Hu et al. 2011; Sun et } \\
\text { al. 2010) }\end{array}$} \\
\hline & & & & 22Rv1 & $\begin{array}{l}\text { 35kb tandem duplication } \\
\text { (exon 3) }\end{array}$ & \\
\hline & & & & VCaP & Amplification & \\
\hline AR-13 & 6,9 & negative & mRNA & & & (Hu et al. 2011) \\
\hline AR-V14 & 7,9 & NA & mRNA & & & (Hu et al. 2011) \\
\hline \multirow{3}{*}{ AR8 } & \multirow{3}{*}{$1 / 3$ /intron3 } & \multirow{3}{*}{$\begin{array}{l}\text { Increases } \\
\text { phosphorylation } \\
\text { of full-length AR }\end{array}$} & \multirow{3}{*}{ mRNA } & C4-2 & & \multirow{3}{*}{ (Yang et al. 2011) } \\
\hline & & & & $\begin{array}{l}\text { C4-2B } \\
\text { CWR22 }\end{array}$ & & \\
\hline & & & & CWR-R1 & 48kb deletion (intron 1) & \\
\hline
\end{tabular}

Constitutive; ligand-independent nuclear localization and activity. Conditional; cell line- or promoter-specific ligand-independent nuclear localization and activity. Negative; no ligandindependent localization or activity. NA, not assessed. 
Table 2. Clinical and biological relevance of $A R$ variants.

\begin{tabular}{|c|c|c|c|}
\hline Variant & Clinical Relevance & Biological Relevance & Refs. \\
\hline AR-V7 & $\begin{array}{l}\text { Enriched in HRPC and metastasis, high risk } \\
\text { of recurrence, decreased overall survival }\end{array}$ & $\begin{array}{l}\text { Resistant to ADT, Resistant } \\
\text { to Enzalutamide, induces } \\
\text { EMT }\end{array}$ & $\begin{array}{l}\text { (Cottard et al. 2013; Guo et } \\
\text { al. 2009; Hu et al. 2009; } \\
\text { Sun et al. 2010; Watson et } \\
\text { al. 2010) }\end{array}$ \\
\hline $\begin{array}{l}\text { AR-V12/ } \\
\text { AR }^{\text {v567es }}\end{array}$ & Enriched in HRPC and metastasis & $\begin{array}{l}\text { Resistant to } A D T \text {, drives } \\
\text { invasive adenocarcinoma in } \\
\text { vivo }\end{array}$ & $\begin{array}{l}\text { (Liu et al. 2013b; Sun et al. } \\
\text { 2010) }\end{array}$ \\
\hline$A R-V 1$ & Enriched in HRPC and metastasis & Not resistant to ADT & $\begin{array}{l}\text { (Hornberg et al. 2011; Hu } \\
\text { et al. 20009; Watson et al. } \\
\text { 2010) }\end{array}$ \\
\hline
\end{tabular}

Hormone-refractory prostate cancer (HRPC); Androgen deprivation therapy (ADT); Epithelial to mesenchymal transition (EMT). 
Table 3. Potential targets for AR variant driven CRPC.

\begin{tabular}{|c|c|c|c|}
\hline AR Variants & Effect & Targets & Compounds \\
\hline $\begin{array}{l}\text { Truncated AR } \\
\text { variants }\end{array}$ & Activate & $A R$ & EPI-001, compound 30, ASC-J9 \\
\hline $\begin{array}{l}\text { Enhancers of } \\
\text { AR Variant } \\
\text { Activity }\end{array}$ & Effect & Targets & Compounds \\
\hline SRFS1, U2AF2 & $\begin{array}{l}\text { Activate } \\
\text { upstream }\end{array}$ & AR cryptic exons & splice switching oligos, RON inhibitors \\
\hline PI3K/AKT & $\begin{array}{l}\text { Activate } \\
\text { upstream }\end{array}$ & $\mathrm{PI3K} / \mathrm{AKT}$ inhibitors & BKM120, MK2206, GDC-0941, others \\
\hline c-Src & $\begin{array}{l}\text { Activate } \\
\text { upstream }\end{array}$ & $\begin{array}{c}\text { AKT inhibitors, c-Src } \\
\text { inhibitors }\end{array}$ & MK2206, Dasatinib, PP2A activators \\
\hline NFkB & $\begin{array}{l}\text { Activate } \\
\text { upstream }\end{array}$ & NFkB inhibitors & statins, bortezomib \\
\hline SRC-1 & Co-activate & $\begin{array}{l}\text { Growth factor receptors, } \\
\text { Erk }\end{array}$ & Vandetanib, BVD-523 \\
\hline $\begin{array}{l}\text { AR Variant } \\
\text { Independent }\end{array}$ & Effect & Targets & Compounds \\
\hline HSP9O & Inhibit upstream & HSP90/27 & STA-9090, AT13387, OGX-427 \\
\hline $\begin{array}{l}\text { Inhibitors of } \\
\text { AR Variant } \\
\text { Activity }\end{array}$ & Effect & Targets & Compounds \\
\hline DAB2IP & Inhibit upstream & $\mathrm{EZH} 2$ inhibitors & E7438 \\
\hline FOXO1 & Inhibit upstream & AKT/PI3K & BKM120, MK2206 \\
\hline $\begin{array}{l}\text { Downstream } \\
\text { AR Variant } \\
\text { Targets }\end{array}$ & Effect & Targets & Compounds \\
\hline RHOB & $\begin{array}{c}\text { Target } \\
\text { downstream }\end{array}$ & Rho, GPCR & statins, Sulindac \\
\hline UBE2C & $\begin{array}{c}\text { Target } \\
\text { downstream }\end{array}$ & cell cycle checkpoint & $\begin{array}{c}\text { ARQ 501, PD-0332991, CDK4/6 inhibitor, docetaxel, } \\
\text { cabazitaxel }\end{array}$ \\
\hline EMT & $\begin{array}{c}\text { Target } \\
\text { downstream }\end{array}$ & HDAC inhibitors & $\begin{array}{c}\text { Vorinostat, N-cadherin antibodies, inhibition of stemness } \\
\text { programs (Gli/Hedgehog, NOTCH), combination } \\
\text { approaches, c-met inhibition, DAB2IP/EZH2 inhibitors, } \\
\text { many others (see (Bitting et al. 2014)) }\end{array}$ \\
\hline
\end{tabular}




\section{DUKE UNIVERSITY MEDICAL CENTER}

CURRICULUM VITAE

Date Prepared: July 9, 2014

Name: Andrew J. Armstrong, MD ScM

Primary academic appointment: Associate Professor, Medicine (Medical Oncology) and Surgery

Primary academic department: Medicine

Secondary appointment: Surgery

Present academic rank and title: Associate Professor with tenure

Date and rank of first Duke Faculty appointment: September 1, 2006

Medical Licensure: North Carolina License \# 2006-01418

Date of License (Month/Day/Year) 08/16/2006

Specialty certification(s) and dates (Month/Day/Year):

Internal Medicine, 2003, expires 12/31/2013, recertified April 23, 2013

Medical Oncology, 2006, expires 12/31/2016

Hematology, 2006, expires 12/31/2016

Date of birth: $02 / 21 / 1974 \quad$ Place: Atlanta, Georgia/USA

Citizen of: United States

\begin{tabular}{|c|c|c|c|}
\hline Education & Institution & Date (Year) & Degree \\
\hline High School & $\begin{array}{l}\text { John Handley High School } \\
\text { Winchester, VA }\end{array}$ & 1988-1992 & $\mathrm{n} / \mathrm{a}$ \\
\hline College & $\begin{array}{l}\text { Duke University School of } \\
\text { Engineering }\end{array}$ & 1992-1996 & $\begin{array}{l}\text { BSE } \\
\text { (distinction) }\end{array}$ \\
\hline \multirow[t]{2}{*}{$\begin{array}{l}\text { Graduate or } \\
\text { Professional School }\end{array}$} & $\begin{array}{l}\text { University of Virginia School of } \\
\text { Medicine }\end{array}$ & 1996-2000 & $\mathrm{MD}$ \\
\hline & $\begin{array}{l}\text { Johns Hopkins School of Public } \\
\text { Health }\end{array}$ & 2004-2008 & $\begin{array}{c}\text { ScM } \\
\text { (Masters of Science) }\end{array}$ \\
\hline
\end{tabular}


Scholarly societies:

Phi Eta Sigma

Golden Key National Honor Society

Tau Beta Pi National Engineering Honor Society

Alpha Omega Alpha

Delta Omega National Public Health Honor Society (nominee)

American College of Physicians (Fellow, FACP)

Professional training and academic career:

Institution

Hospital of the University of Pennsylvania

Johns Hopkins School of Medicine

Johns Hopkins School of Public Health

Graduate Training Program in Clinical

Investigation (GTPCI)

Duke University Department of Medicine

Duke University Department of Medicine

Duke University Department of Medicine

\author{
Position/Title \\ Intern and Resident \\ Oncology and Hematology Fellow
}

Research Fellow

Assistant Professor

of Medicine and Surgery

Associate Professor

of Medicine and Surgery

Associate Professor with tenure

of Medicine and Surgery $\frac{\text { Dates }}{2000-2003}$
$2003-2006$

2004-2008

2006-2011

2012-present

2014-present

\section{Mission Statement}

As a clinical and translational investigator, I have spent the last 7 years investigating tumor-host interactions in the context of experimental therapeutics for patients with advanced genitourinary malignancies, particularly with a focus on prostate cancer and the investigation of biomarkers of response and benefit. It is in this context that my research focus has turned to the investigation of circulating tumor cell biology, with a focus on epithelial-mesenchymal transitions in these cells and how this may relate to the lethal phenotype. This work has led to a Department of Defense Physician Research Training Award and Prostate Cancer Foundation Young Investigator Award, and findings confirming EMT marker (N-cadherin, vimentin) expression in CTCs from these patients with metastatic cancer, as well as evidence of stem cell biomarker expression on these cells. These findings implicate epithelial plasticity in treatment resistance and metastatic dissemination in prostate cancer and also imply that CTCs inhabit a transitional or intermediate state and may possess stemlike properties. I have developed a number of experimental agents in prostate and renal cell cancer, including completed or ongoing trials of mTOR inhibitors and PI3 kinase inhibitors, immunomodulatory agents, hormonal therapies, and anti-angiogenic agents, and am heavily involved in the leadership of several phase 3 studies in advanced prostate cancer (dasatinib, tasquinimod, enzalutamide) in CRPC. I co-direct the Duke clinical research program in genitourinary malignancies, overseeing 8 research nurses, 4 clinical trial assistants, 2 regulatory specialists, and 2 budget and finance personnel, and provide mentorship to medical oncology fellows and post-doctoral laboratory based fellows. I am the principal investigator on 8 investigator-initiated clinical trials and approximately 12 industry or cooperative group sponsored clinical trials, as well as several correlative science studies and am an internationally recognized expert in prognostic and predictive biomarkers and outcome studies in men with castration-resistant metastatic disease. 


\section{Publications:}

${ }^{*}=$ notable peer reviewed first/senior publications selected

\section{Peer Reviewed First/Senior Author Publications (in order of chronicity):}

1. Armstrong AJ, Eck SJ. EpCAM: A New Therapeutic Target for an Old Cancer Antigen. Cancer Biology and Therapy. 2003; 2(4): 320-5.

*2. Armstrong AJ*, Garrett-Mayer ES, Yang YC, de Wit R, Tannock IF, Eisenberger M. A contemporary prognostic nomogram for men with hormone-refractory metastatic prostate cancer (HRPC). Clin Cancer Research 2007;13:6396-6403.

Description: Developed an internally validated prognostic nomogram for men undergoing chemotherapy for castrationresistant prostate cancer. This nomogram has the highest predictive accuracy as compared to other available prognostic models in this disease and is widely recognized and used internationally.

*3. Armstrong AJ*, Garrett-Mayer E, Ou Yang YC, Carducci MA, Tannock I, de Wit R, Eisenberger M. PSA and pain surrogacy analysis in men with metastatic hormone-refractory prostate cancer (HRPC). J Clin Oncol 2007;29:3965-70.

Description: This paper refuted the evidence that declines in PSA following docetaxel therapy were surrogate endpoints (ie potential approvable endpoints) in men with CRPC, and also evaluated changes in pain. These data helped provide evidence that informed on the Prostate Cancer Working Group's criteria for endpoints in clinical trials, and fortified evidence that overall survival should remain the primary phase 3 endpoint in this disease.

4. Armstrong AJ*, George DJ. Satraplatin in the Treatment of Hormone-Refractory Metastatic Prostate Cancer. Ther Clin Risk Mgmt 2007; 3(5)

5. Armstrong AJ*, Creel P, Turnbull J, Moore C, Jaffe TA, Haley S, Petros W, Yenser S, Gockerman JP, Sleep D, Hurwitz H, George DJ. A phase I-II study of docetaxel and atrasentan in men with castration resistant metastatic prostate cancer. Clin Cancer Res 2008;14: 6270-76.

Description: This was a phase 1-2 report of the novel endothelin-A receptor inhibitor with docetaxel in men with CRPC, and provided evidence for an independent bone turnover modifying property of this agent; these data also set the stage for the eventual and ongoing phase 3 trial being currently conducted by the NCl cooperative groups.

6. Armstrong AJ*, Halabi S, de Wit R, Tannock IF, Eisenberger M. The relationship of body mass index and serum testosterone levels with disease outcomes in castration-resistant metastatic prostate cancer. Prostate Cancer and Prostatic Diseases 2008; 36:1-6.

7. Armstrong AJ*, Tannock IF, de Wit R, George DJ, Eisenberger M, Halabi S. The development of risk groups in men with metastatic castration-resistant prostate cancer based on predictive risk factors for PSA decline and survival. Eur J Cancer 46:517-525, 2010.

8. Armstrong AJ* and PG Febbo. Using Surrogate Biomarkers to Predict Clinical Benefit in Men with Castration-Resistant Prostate Cancer: an Update and Review of the Literature. Oncologist 2009, 14: 816-27.

*9. Armstrong AJ*, Garrett-Mayer E, Tannock IF, de Wit R, and M Eisenberger. Prediction of Survival Following First Line Chemotherapy in Men with Castration-Resistant Metastatic Prostate Cancer, Clin Cancer Res, 2010;1:203-211.

Description: This is the first post-docetaxel nomogram to be developed, and has reasonable prognostic accuracy, incorporating several novel factors such as type of progression and duration of first line chemotherapy. The clinical utility of this nomogram in the post-docetaxel space has increased following the approval of novel agents in this setting in 2010. 
10. Armstrong AJ*, George DJ. Optimizing Docetaxel Chemotherapy for Men with Metastatic Castration Resistant Prostate Cancer. Prostate Cancer Prostatic Diseases 2010 (published online Jan 12)

*11. Armstrong AJ*, Netto GJ, Rudek MA, Halabi S, Wood D, Creel P, Mundy K, Davis SL, Wang T, Albadine R, Schultz L, Partin A, Jimeno A, Fedor H, Febbo PG, George DJ, Gurganus R, DeMarzo AM, Carducci MA. Pharmacodynamic study of pre-prostatectomy rapamycin in men with advanced localized prostate cancer. Clin Cancer Res 2010;16: 3057-66.

Description: This was a rigorous mechanistic study of the mTOR inhibitor rapamycin in men with localized prostate cancer that utilized paired tumor biopsy specimens, PK and PBMC data, and found that while rapamycin inhibited its intended downstream target, it had no effect on proliferation or apoptosis, thus providing the only published evidence of clinical mechanism of this class of agents in prostate cancer and suggesting methods to improve upon targeted therapy against the PI3K pathway in prostate cancer.

12. Sonpavde G, Pond GR, Berry WR, de Wit R, Eisenberger MA, Tannock IF, and AJ Armstrong*. The association between radiographic response and overall survival in men with metastatic castration-resistant prostate cancer receiving chemotherapy. Cancer 2011 (online ahead of press March 1, 2011)

13. Bitting R, Madden J, and Armstrong AJ*. Therapy for non-clear cell histologies in renal cancer. Curr Clin Pharmacol 2011, Epub ahead of press Aug 9, 2011.

14. Pili R, Häggman M, Stadler WM, Gingrich J, Assikis V, Björk A, Nordle Ö, Forsberg G, Carducci MA, Armstrong, A.J. Phase II randomized double blind placebo-controlled study to determine the efficacy of tasquinimod in asymptomatic patients with metastatic castrate-resistant prostate cancer. J Clin Oncol 2011 Sept 19 (epub ahead of press)

This large international phase 2 trial of the novel agent tasquinimod demonstrated a more than doubling of the progression-free survival of men with metastatic CRPC over placebo and has led to the launch of a global phase 3 registrational study. This agent is an S100A9 inhibitor with effects on angiogenesis and myeloid-derived suppressor cell function, each of which apparently has anti-tumor and anti-metastatic functions.

15. Antonarakis $A$ and Armstrong AJ*. Evolving standards in the treatment of castration-resistant metastatic prostate cancer. Prostate Cancer Prostatic Diseases. 2011; 14(3):192-205

16. Antonarakis A and Armstrong AJ*. Emerging therapeutic approaches in the management of men with metastatic castration-resistant prostate cancer. Prostate Cancer Prostatic Diseases. 2011;14(3):206-18.

*17. Armstrong AJ*, Kemeny G, Marengo M, Oltean S, Chen L, Herold C, Turnbull J, Marcom PK, George DJ, and Garcia-Blanco MA*. Circulating Tumor Cells from Patients with Advanced Prostate and Breast Cancer Display Both Epithelial and Mesenchymal Markers. Mol Cancer Res 2011 (epub ahead of print July 26, 2011)

*= co-corresponding author

This correlative study of human circulating tumor cells establishes the presence of markers of epithelial mesenchymal transition in CTCs taken from patients with metastatic breast and prostate cancer, as well as provides evidence for the expression of stemness antigens and epithelial plasticity in metastatic sites.

18. Pond G*, Armstrong AJ*, Wood BA, Brookes M, Leopold L, Berry WR, de Wit R, Tannock IF, Sonpavde G. Evaluating the Value of Number of Cycles of Docetaxel and Prednisone in Men with Metastatic CastrationResistant Prostate Cancer. Eur Urol 2011 (Epub ahead of print 6/22/11).

*=co-first author

19. Armstrong AJ*, Eisenberger MA, Halabi S, Oudard S, Nanus DM, Petrylak DP, Sartor AO, Scher HI. Biomarkers in the management and treatment of men with metastatic castration-resistant prostate cancer. Eur Urol 2011 (Epub ahead of print November 12, 2011). 
20. Armstrong AJ*, George DJ, Halabi S. Serum lactate dehydrogenase (LDH) as a predictive biomarker of overall survival with mTOR inhibition in patients with metastatic renal cell carcinoma (RCC). J Clin Oncol 2011, Epub ahead of press Aug 13, 2012.

21. Pond GR, Armstrong AJ*, Wood BA, Leopold L, Galsky MD, Sonpavde G. Ability of c-reactive protein to complement multiple prognostic classifiers in men with metastatic castration resistant prostate cancer receiving docetaxel-based chemotherapy. BJUI 201, Epub ahead of print April 23, 2012.. ${ }^{*}=$ co-first author

22. Pond GR, Armstrong AJ*, Galsky MD, Wood BA, Leopold L, Sonpavde G. Efficacy of docetaxel-based chemotherapy following ketoconazole in metastatic castration-resistant prostate cancer. Urol Oncol 2012 (epub ahead of print May 1, 2012). * $=$ co-first author

23. Turnbull, JD, Cobert J, Jaffe T, Harrison MR, George DJ, Armstrong AJ*. Activity of single agent bevacizumab in patients with metastatic renal cell carcinoma previously treated with vascular endothelial growth factor tyrosine kinase inhibitors. Clin Genitourin Cancer 2012; Epub ahead of print Oct 4, 2012.

24. Armstrong AJ*. The STAMPEDE Trial and Celecoxib: How to Adapt? Lancet Oncol, epub March 26, 2012.

25. Sonpavde G, Armstrong AJ. Objective evaluation of bone metastases in prostate cancer: to what end? Eur Urol: epub ahead of print Feb 20, 2012.

26. Scher HI, Fizazi K, Saad F, Taplin ME, Sternberg CN, Miller K, de Wit R, Pulders P, Chi KN, Shore ND, Armstrong AJ, Flaig TW, Flechon A, Mainwaring P, Fleming M, Hainsworth JD, Hirmand M, Selby B, Seely L, de Bono JS. Enzalutamide prolonged survival in men with prostate cancer following chemotherapy. N Engl J Med 2012, Epub ahead of press Aug 15, 2012.

27. Bitting R, Boominathan R, Rao C, Kemeny G, Foulk B, Garcia-Blanco MA, Connelly M, and Andrew J. Armstrong*. Development of a method to isolate circulating tumor cells using mesenchymal-based capture. Methods. 2013 (online ahead of press July 9). *corresponding author

28. Bitting RL, Somarelli JA, Schaeffer D, Garcia-Blanco MA, and AJ Armstrong*. The Role of Epithelial Plasticity in Prostate Cancer Dissemination and Treatment Resistance. Cancer and Metastasis Rev Epub ahead of press Jan 11, 2014. *corresponding author

29. Armstrong AJ*, Haggman M, Stadler WM, Gingrich J, Assikis V, Polikoff J, Damber JE, Belkoff L, Nordle O, Forsberg G, Carducci MA, Pili R. Long term survival and biomarker correlates of tasquinimod efficacy in a multicenter randomized study of men with minimally symptomatic metastatic castration-resistant prostate cancer. Clin Cancer Res, 2013, online ahead of press Dec 15.

30. Bitting RL, Armstrong AJ*. Targeting the PI3K/Akt/mTOR pathway in castration-resistant prostate cancer. Endocr Relat Cancer. 2013; 20(3): R83-99.

31. Armstrong AJ*, Shen $T$, Halabi S, Kemeny G, Bitting RL, Kartcheske P, Embree E, Morris K, Winters C, Jaffe T, Fleming M, George DJ. A Phase II Trial of Temsirolimus in Men With Castration-Resistant Metastatic Prostate Cancer. Clin Genitourin Cancer. 2013, July 3 Epub ahead of print.

32. Zhang, T and Armstrong, A.J*. Clinical subtypes of castration resistant prostate cancer. Clin Adv Hematol Oncol. 2013, 11(11): 707-18.

33. Ware K, Armstrong AJ, Garcia-Blanco MA, Dehm S. Biologic and Clinical Relevance of Androgen Receptor (AR) Variants in Castration Resistant Prostate Cancer (CRPC). Endocrin Rel Cancer 2013 (online ahead of press May 23, 2014). 
34. Pond GR, Sonpavde G, de Wit R, Eisenberger MA, Tannock IF, Armstrong AJ*. The Prognostic Importance of Metastatic Site in Men with Metastatic Castration-resistant Prostate Cancer. Eur Urol 2014, Epub ahead of press Jan; 65: 3-6. *corresponding author

35. Harrison $\mathrm{MH}$, Armstrong AJ. Burden of Disease Matters When It Comes to Systemic Therapy for Prostate Cancer. Eur Urol 2014 (Epub ahead of press march 4, 2014)

37. Aggarwal R, Zhang T, Small EJ, Armstrong AJ. Neuroendocrine prostate cancer: subtypes, biology, and clinical outcomes. J Natl Compr Canc Netw 2014; May; 12(5): 719-26.

38. Sonpavde G, Wang CG, Galsky MD, Oh WK, Armstrong AJ*. Cytotoxic chemotherapy in the contemporary management of metastatic prostate cancer. BJUI 2014 (in press). * corresponding author.

\section{Additional Peer Reviewed Publications}

1. Fukasawa K, Zhou R, Matten W, Armstrong AJ, Daar I, Oskarsson M, Sathyanarayana BK, Maclvor L, Wood TG, Vande Woude GF. Mutagenic Analysis of Functional Domains of the mos Proto-oncogene and Identification of the Sites Important for MAPK Activation and DNA Binding. Oncogene. 1995; 11: 1447-1457.

2. Nightingale RW, Camacho DL, Armstrong AJ, Robinette JJ, Myers BS. Inertial Properties and Loading Rates Affect Buckling Modes and Injury Mechanisms in the Cervical Spine. Journal of Biomechanics. 2000; 33: $191-7$.

3. Armstrong AJ, Eisenberger $\mathrm{M}$. The risk of clinical fractures after gonadotropin-releasing hormone agonist therapy for prostate cancer. Nature Clin Pract Urol 2006; 3:246-7.

4. Armstrong AJ, Garrett-Mayer ES, Eisenberger MA. Adaptive therapy for androgen-independent prostate cancer. J Natl Cancer Inst 2008;100:681-3.

5. Araujo JC, Mathew P, Armstrong AJ, Braud EL, Posadas E, Lonberg M, Gallick GE, Trudel GC, Paliwal P, Agrawal S, and Logothetis CJ. Dasatinib combined with docetaxel for castration-resistant prostate cancer: results from a phase 1/2 study. Cancer 2011 March (epub ahead of press)

6. Sonpavde G, Pond GR, Berry WR, de Wit R, Armstrong AJ, Eisenberger MA, Tannock IF. Serum alkaline phosphatase changes predict survival benefit independent of PSA changes in men with castrationresistant prostate cancer and bone metastasis receiving chemotherapy. Urol Oncol 2010 Sept 29 (epub ahead of press $9 / 29 / 10$ )

7. Whang Y, Armstrong AJ, Rathmell WK, Godley PA, Kim WY, Pruthi RS, Wallen EM, Crane JM, Moore DT, Grigson G, Morris K, Watkins CP, George DJ. A phase 2 study of lapatinib, a dual EGFR and HER2 tyrosine kinase inhibitor, in patients with castration resistant prostate cancer. Urol Oncol 2013 (online ahead of press March 9, 2011)

8. Stewart SB, Bañez LL, Robertson CN, Freedland SJ, Polascik TJ, Xie DH, Koontz BF, Vujaskovic Z, Lee WR, Armstrong AJ, Febbo PG, George DJ, and Moul JW. Utilization Trends of a Multidisciplinary Prostate Cancer Clinic: Initial 5-Year Experience from the Duke Prostate Center. J Urol. January 2012. Epub ahead of print Nov 14, 2011.

9. Bitting RL, Armstrong AJ, and Daniel J George. Management options in advanced prostate cancer: what is the role for sipuleucel-T? Clin Medicine Insights: Oncology 2011; 5: 325-332.

10. Peppercorn, J, Armstrong AJ, Zaas DW, George DJ. Rationing in urologic oncology: lessons from sipuleucel-T for advanced prostate cancer. Urol Oncol 2012, Epub ahead of print Feb 3, 2012.

11. Saylor PJ, Armstrong AJ, Fizazi K, Freedland SJ, Saad F, Smith MR, Tombal B, Pienta K. New and 
emerging therapies for bone metastases in genitourinary cancers. Eur Urol 2012 epub ahead of press Nov 23, 2012.

12. Harrison MR, Wong TZ, Armstrong AJ, George DJ. Radium-223 chloride: a potential new treatment for castration-resistant prostate cancer patients with metastatic bone disease. Cancer Management and Research 2012, Epub ahead of print Jan 8, 2013.

13. Noonan KL, North S, Bitting RL, Armstrong AJ, Ellard S, Chi KN. Clinical activity of abiraterone acetate in patients with metastatic castration-resistant prostate cancer progressing after enzalutamide. Annals of Oncology 2013 Epub ahead of press April 12, 2013.

14. Bitting RL, Armstrong AJ. Targeting the PI3K/Akt/mTOR pathway in castration-resistatnt prostate cancer. Endocr Relat Cancer 2013, May 1.

15. Schweizer MT, Lin J, Blackford A, Bardia A, King S, Armstrong AJ, Rudek MA, Yegnasubramanian S, Carducci MA. Pharmacodynamic study of disulfiram in men with non-metastatic recurrent prostate cancer. Prostate Cancer Prostatic Dis. 2013, Aug 20 Epub ahead of print.

16. Araujo JC, Trudel GC, Saad F, Armstrong AJ, Yu EY, Bellmunt J, Wilding G, McCaffrey J, Serrano SV, Matveev V, Efstathiou E, Oudard S, Morris MJ, Sizer B, Goebell PJ, Heidenreich A, de Bono JS, Begbie S, Hong JH, Richardet E, Gallardo E, Paliwal P, Durham S, Cheng S, Logothetis C. Randomized, Double-Blind, Placebo-Controlled Phase 3 Trial of Docetaxel and Dasatinib in Men with Metastatic Castration-Resistant Prostate Cancer. Lancet Oncol. October 2013, online ahead of press Nov 8.

17. Bitting RL, MD; Healy P; Creel PA; Turnbull J; Morris K; Yenser Wood S; Hurwitz HI; Starr MD; Nixon AB; Armstrong AJ; George DJ. A Phase lb Study of Combined VEGFR and mTOR Inhibition with vatalanib and everolimus in Patients with Advanced Renal Cell Carcinoma. Clin Genitourin Cancer 2013 (in press).

18. Ware K, Garcia-Blanco MA, Armstrong AJ, Dehm S. Significance of Androgen Receptor Variants in Castration Resistant Prostate Cancer. Endocr Rel Cancer 2013 (online ahead of press May 23, 2014).

19. Halabi S, Lin CY, Small EJ, Armstrong AJ, Kaplan EB, Petrylak D, Sternberg CN, Shen L, Oudard S, de Bono J, Sartor O. Prognostic Model Predicting Metastatic Castration-Resistant Prostate Cancer Survival in Men Treated With Second-Line Chemotherapy. J Natl Cancer Inst 2013 Oct 17 Epub ahead of press.

20. Halabi S, Armstrong AJ, Sartor O, de Bono J, Kaplan E, Lin CY, Solomon NC, Small EJ. ProstateSpecific Antigen Changes As Surrogate for Overall Survival in Men With Metastatic Castration-Resistant Prostate Cancer Treated With Second-Line Chemotherapy. J Clin Oncol 2013 Oct 7 Epub ahead of print.

21. Stoyanova T, Cooper AR, Drake JM, Liu X, Armstrong AJ, Pienta KJ, Zhang H, Kohn DB, Huang J, Witte ON, Goldstein AS. Prostate cancer originating in basal cells progresses to adenocarcinoma propagated by luminal-like cells. Proc Natl Acad Sci USA 2013, online ahead of press Dec 10.

22. Sonpavde G, Pond GR, Armstrong AJ, Galsky MD, Leopold L, Wood BA, Wang SL, Paolini J, Chen I, Chow-Maneval E, Mooney DJ, Lechuga M, Smith MR, Michaelson MD. Radiographic progression by Prostate Cancer Working Group (PCWG)-2 criteria as an intermediate endpoint for drug development in metastatic castration resistant prostate cancer. BJUI Int 2013, Epub ahead of press Dec 3.

23. Mohler JL, Kantoff PW, Armstrong AJ, Bahnson RR, Cohen M, D'Amico AV, Eastham JA, Enke CA, Farrington TA, Higano CS, Horwitz EM, Kawachi MH, Kuettel M, Lee RJ, Macvicar GR, Malcolm AW, Miller D, Plimack ER, Pow-Sang JM, Richey S, Roach M 3rd, Rohren E, Rosenfeld S, Small EJ, Srinivas S, Stein C, Strope SA, Tward J, Walsh PC, Shead DA, Ho M. Prostate cancer, version 1.2014. J Natl Compr Canc Netw. 2013 Dec 1;11(12):1471-9. 
24. Younis I, George D, McManus T, Hurwitz H, Creel P, Armstrong AJ, Yu J, Bacon K, Hobbs, G, Peer C, Petros W. Clinical pharmacology of an atrasentan and docetaxel regimen in men with hormone-refractory prostate cancer. Cancer Chemo Pharmacol 2014 (in press).

25. Beer TM, Armstrong AJ, Rathkopf DE, Loriot $Y$, Sternberg CN, Higano CS, Iversen $P$, Bhattacharya $S$, Carles J, Chowdhury S, Davis ID, de Bono JS, Evans CP, Fizazi K, Joshua AM, Kim C, Kimura G, Mainwaring P, Mansbach H, Miller K, Noonberg SB, Perabo F, Phung D, Saad F, Scher HI, Taplin ME, Venner PM, and Tombal B. Benefit of Enzalutamide in Chemotherapy-Naïve Metastatic Prostate Cancer. N Engl J Med 2014 (online ahead of press June 1, 2014).

26. Shuch B, Amin A, Armstrong AJ, Eble JN, Ficarrae V, Lopez-Beltran A, Martignoni G, BI Rini, A Kutikov. Understanding Pathologic Variants of Renal Cell Carcinoma: Distilling Therapeutic Opportunities from Biologic Complexity. Eur Urol 2014 (online ahead of press April 29, 2014).

\section{Non-refereed review publications:}

1. Armstrong AJ, Carducci MA. Novel therapeutic approaches to advanced prostate cancer. Clin Adv Hematol Oncol 2005;3: 271-282.

2. Armstrong AJ, Carducci MA. Chemotherapy for advanced prostate cancer: results of new clinical trials and future studies. Curr Oncol Reports 2005; 7:110-7.

3. Armstrong AJ, Carducci MA. Advanced prostate cancer: the future. Can J Urol 2005; 12(Suppl 1): 78-83.

4. Armstrong AJ, Carducci MA. New Drugs for Prostate Cancer. Curr Opin Urol 2006; 16: 138-45.

5. Mendiratta $\mathrm{P}$, Armstrong AJ, George DJ. Advances in the management of advanced prostate cancer. Rev Urol 2007; 9 (Suppl 1): S9-S19.

6. Srinivasan R, Armstrong AJ, Dahut W, and George DJ. Anti-angiogenic therapy in renal cell cancer. Br J Urol 2007; 99: 1296-1300.

7. Armstrong AJ, Febbo PG, George DJ, Moul JW. Systemic strategies for prostate cancer. Minerva Urol Nefrol 2007:59:11-25.

8. Armstrong AJ, George DJ. New drug development in metastatic prostate cancer. Semin Urol Oncol 2008 26: $430-7$.

9. Ramiah V, George DJ, Armstrong AJ. Clinical endpoints for drug development in prostate cancer. Curr Opin Urol 2008;18: 303-8.

10. Chen FL, Armstrong AJ, George DJ. Cell signaling modifiers in prostate cancer. Cancer J 2008;14: 4045.

11. Figlin RA, Brown E, Armstrong AJ, Akerley W, Benson AB 3rd, Burstein HJ, Ettinger DS, Febbo PG, Fury MG, Hudes GR, Kies MS, Kwak EL, Morgan RJ Jr, Mortimer J, Reckamp K, Venook AP, Worden F, Yen Y. NCCN Task Force Report: mTOR inhibition in solid tumors. JNCCN 2008; 6(5): S1-S23.

12. Armstrong AJ, Freedland SJ, Garcia-Blanco M. Epithelial-mesenchymal transition in prostate cancer: providing new targets for therapy. Asian J Androl 2010, published online Dec 2010; 12:179-80.

13. Antonarakis $\mathrm{E}$ and Armstrong AJ. Changing paradigms in the managements of metastatic castrationresistant prostate cancer. Clinical Oncology News 2011.

14. Bitting R, Armstrong AJ, George DJ. Management options in advanced prostate cancer: what is the role 
for sipuleucel-T? Clin Med Insights 2011 (in press).

15. Armstrong AJ, Ferrari AC, Quinn DI. The role of surrogate markers in the management of men with metastatic castration-resistant prostate cancer. Clin Adv Hematol Oncol 2011.

16. Sonpavde G and Armstrong AJ. Objective measurement of bone metastases in prostate cancer: to what end? Eur Oncol 2012 (Epub ahead of print Feb 20, 2012)

17. Armstrong AJ, Moul JW, George DJ. What to order from the prostate treatment menu? 2012; $84: 87-88$.

18. Bitting RL, Armstrong AJ. Prognostic, predictive, and surrogate factors for individualizing treatment for men with castration-resistant prostate cancer. In: Govindan R, ed 2012 ASCO Educational Book. Alexandria, VA: American Society of Clinical Oncology; 2012; 292-297.

19. Bitting RL, Armstrong AJ. Potential predictive biomarkers for individualizing treatment for men with CRPC. Cancer J, 19: 25-33, Jan 2013.

20. Clarke JM, Armstrong AJ. Novel therapies for the treatment of advanced prostate cancer. Curr Treat Oncol, 14: 109-26, 2013.

21. Armstrong AJ. New Treatments for Men with Castration-resistant Prostate Cancer: Can We Move from Small Steps to Giant Leaps? Eur Urol 2013, epub ahead of press Sept 10, 2013.

\section{Chapters in books:}

1. Armstrong AJ, Carducci MA. Chemotherapy for advanced prostate cancer. In: Principles and Practice of Prostate Cancer. First Edition. Edited by Kirby RS, Partin A, Feneley M, Parsons JK. London: Martin Dunitz; 2005.

2. Armstrong AJ, Carducci MA. Chemotherapy Strategies for Advanced Disease. In: Therapeutic Strategies in Prostate Cancer. First Edition. Edited by Mark R. Feneley and Heather A.Payne. London: Clinical Publishing, 2007.

3. Kim W, Armstrong AJ, George DJ. Akt pathway biology in renal cell carcinoma. In: Renal Cell Carcinoma. First Edition. Edited by Brian Rini and Steven Campbell. Ontario: BC Decker, Inc., 2007 (in press)

4. Moul JW, Armstrong AJ, Hollenbeck BK, Lattanzi J, Bradley D, and Hussain M: Prostate Cancer. In: R Pazdur, LD Wagman, KA Camphausen, and WJ Hoskins (Eds). 11th Edition Cancer Management: A Multidisciplinary Approach, CMP Healthcare Media, Manhasset, NY, Chapter 17, pp. 393-423, 2008.

5. Moul JW, Armstrong AJ, Hollenbeck BK, Lattanzi J, Bradley D, and Hussain M: Prostate Cancer. In: R Pazdur, LD Wagman, KA Camphausen, and WJ Hoskins (Eds). 12th Edition Cancer Management: A Multidisciplinary Approach, CMP Healthcare Media, Manhasset, NY, Chapter 15, 2009.

6-7. Mendiratta P, Armstrong AJ. Genitourinary Cancers. In: Oxford-American Handbook of Oncology. Ed: Gary Lyman. Chapters 19, 21-24, 2009 and 2010.

8. Mendiratta P, George DJ, Armstrong AJ. Renal Cell Carcinoma. In: Oxford-American Handbook of Oncology. Ed: Gary Lyman. Chapter 20, 2009.

9-11. Moul JW, Armstrong AJ, Lattanzi J: Prostate Cancer. In: R Pazdur, LD Wagman, KA Camphausen, and WJ Hoskins (Eds). 13th Edition Cancer Management: A Multidisciplinary Approach, CMP Healthcare Media, Manhasset, NY, Chapter 14, 2010. Editions in 2012 and 2013 were also published as a co-author. 
12. Section editor for (section 6), Textbook of Prostate Cancer (Springer), editor: Ashutosh Tewari, in press 2013. Involves editing and oversight for 9 chapters.

13. Li J, Armstrong AJ. Prognostic and Predictive Biomarkers for Castration Resistant Prostate Cancer. In: Biomarkers in Disease: Methods, Discoveries and Applications. 2014 anticipated publication. In press.

14. Zhang T, Armstrong AJ. Evolution of Clinical States and the Castration Resistant Clinical Paradigm. In: Management of Castration Resistant Prostate Cancer, editors: Saad F and Eisenberger MA, 2014 (in press).

\section{a. Published scientific reviews (for mass distribution)}

1. Armstrong AJ, Carducci MA. Chemotherapy for advanced prostate cancer: results of new clinical trials and future studies. Curr Oncol Reports 2005; 7:110-7.

2. Armstrong AJ, Carducci MA. New Drugs for Prostate Cancer. Curr Opin Urol 2006; 16: 138-45.

3. Figlin RA, Brown E, Armstrong AJ, Akerley W, Benson AB 3rd, Burstein HJ, Ettinger DS, Febbo PG, Fury MG, Hudes GR, Kies MS, Kwak EL, Morgan RJ Jr, Mortimer J, Reckamp K, Venook AP, Worden F, Yen Y. NCCN Task Force Report: mTOR inhibition in solid tumors. JNCCN 2008; 6(5): S1-S23.

4. Armstrong AJ. Where Does Abiraterone Fit into the Metastatic Prostate Cancer Treatment Algorithm? Community Oncology, 2012.

5. Harrison MR, Wong TZ, Armstrong AJ, George DJ. Alpharadin: a potential new treatment for castrationresistant prostate cancer patients with metastatic bone disease. Cancer Management and Research, 2012 (in press)

6. Mohler JL, Armstrong AJ, Bahnson RR, Boston B, Busby JE, D'Amico AV, Eastham JA, Enke CA, Farrington T, Higano CS, Horwitz EM, Kantoff PW, Kawachi MH, Kuettel M, Lee RJ, Macvicar GR, Malcolm AW, Miller D, Plimack ER, Pow-Sang JM, Roach M 3rd, Rohren E, Rosenfeld S, Srinivas S, Strope SA, Tward J, Twardowski P, Walsh PC, Ho M, Shead DA. Prostate Cancer, Version 3.2012 Featured Updates to the NCCN Guidelines. J Natl Compr Canc Net 2012, Sept 1; 10(9): 1081-1087.

See above list for non-refereed publications as well.

\section{b. Selected abstracts}

1. Armstrong AJ, Ou Yang YC, Garrett-Mayer E, Carducci MA. Continuation of docetaxel is associated with improved survival beyond disease progression in men with metastatic hormone-refractory prostate cancer. Abstract presented at Johns Hopkins Fellow Research Day, May 2006.

2. Armstrong AJ, Kulesza $P$, Netto GJ, Rudek MA, Halabi S, Wood D, Creel P, Mundy K, Davis SL, Wang T, Albadine R, Schultz L, Partin A, Jimeno A, Fedor H, Febbo PG, George DJ, Gurganus R, DeMarzo AM, Carducci MA. A pharmacodynamic study of pre-prostatectomy rapamycin in men with advanced localized prostate cancer. Abstract presented at Cancer Education Consortium, Amelia Island April 2006.

3. Stoker CE, Adams RB, Slack JB, Armstrong AJ, Parsons JT. Integrin Mediated Migration of Prostate Cancer Cell Lines. Abstract presented at Keystone Symposium on Motility and Metastasis, Copper Mountain, CO, Feb. 25, 1998.

4. Nightingale RW, Camacho DL, Armstrong AJ, Robinette JJ, Myers BS. Cervical Spine Buckling: the Effects of Vertebral Mass and Loading Rate. Presented at 1997 Advances in Bioengineering Conference 
(ASME), vol BED-36, 231-2.

5. Armstrong AJ, de Marzo A, Partin AW, Rudek M, Gurganus R, Beekman K, Hidalgo M, Carducci MA. A pharmacodynamic study of pre-prostatectomy rapamycin in men with advanced localized prostate cancer. Cancer Education Symposium 2006, Amelia Island, Florida (abstract).

6. Armstrong AJ, E. S. Garrett-Mayer, Y. Ou Yang, R. de Wit, I. Tannock and Eisenberger ME. A baseline prognostic model and nomogram incorporating PSA kinetics in hormone-refractory metastatic prostate cancer (HRPC). Proceedings of the American Society of Clinical Oncology Prostate Cancer Symposium 2007, abstract 222.

7. Armstrong AJ, Garrett-Mayer E, Ou Yang Y, Tannock IF, de Wit R, and Eisenberger R. Limitations of the current progression-free survival (PFS) definition in hormone-refractory prostate cancer (HRPC): Benefit associated with continuation of docetaxel beyond TAX327 protocol-defined progression. Proceedings of the American Society of Clinical Oncology Prostate Cancer Symposium 2007, abstract 223.

8. Armstrong AJ, Garrett-Mayer E, Ou Yang YC, Carducci MA, Tannock I, de Wit R, Eisenberger M. Analysis of PSA decline as a surrogate for overall survival in metastatic hormone-refractory prostate cancer (HRPC). Proceedings of the American Society of Clinical Oncology Prostate Cancer Symposium 2007, abstract 148.

9. Speca J, Mears A, Creel T, Armstrong AJ, George DJ. Phase I study of PTK787/ZK222584 (PTK/ZK) and RAD001 for patients with advanced solid tumors and dose expansion in renal cell carcinoma patients. Proc Am Soc Clin Oncol 2007, abstract 5039.

10. Armstrong AJ, E. S. Garrett-Mayer, Y. Ou Yang, R. de Wit, I. Tannock and M. Eisenberger. Analysis of PSA decline as a surrogate for overall survival in metastatic hormone-refractory prostate cancer (HRPC). Proceedings of the American Society of Clinical Oncology 2007, abstract 5009.

11. Armstrong AJ, E. S. Garrett-Mayer, Y. Ou Yang, R. de Wit, I. Tannock and M. Eisenberger.. A multivariate prognostic nomogram incorporating PSA kinetics in hormone-refractory metastatic prostate cancer (HRPC). Proceedings of the American Society of Clinical Oncology 2007, abstract 5058.

12. George DJ, A. J. Armstrong, P. Creel, K. Morris, J. Madden, J. Turnbull, M. Dewhirst, N. Major, P. G. Febbo. A phase 2 study of RAD001 in men with hormone-refractory metastatic prostate cancer. GU Symposium (ASCO) 2008, abstract 181.

13. Armstrong AJ, S. Halabi, I. F. Tannock, de Wit R, and M. A. Eisenberger. The relationship of body mass index and serum testosterone levels with disease outcomes in castration-resistant metastatic prostate cancer. GU Oncology Symposium 2008, abstract 44.

14. Y. E. Whang, C. N. Moore, A. J. Armstrong, W. K. Rathmell, P. A. Godley, J. M. Crane, G. I. Grigson, K. Morris, C. P. Watkins, and D. J. George. Phase II trial of lapatinib in hormone refractory prostate cancer. GU Symposium 2008, abstract 156.

15. G. Netto, A. Armstrong, D. Wood, P. Creel, A. Partin, A. Jimeno, M. Rudek, D. George, R. Gurganus, and M. A. Carducci. Pharmacodynamic (PD) study of pre-prostatectomy rapamycin in men with advanced localized prostate cancer (PC): preliminary results of a Prostate Cancer Clinical Trials Consortium Trial, ASCO 2009 abstract 5001.

16. Armstrong AJ, S. Halabi, I. F. Tannock, D. J. George, R. DeWit, and M. Eisenberger. Development of risk groups in metastatic castration-resistant prostate cancer (mCRPC) to facilitate identification of active chemotherapy regimens, ASCO 2009 abstract 5137. 
17. J. Araujo, A. J. Armstrong, E. L. Braud, E. Posadas, M. Lonberg, G. E. Gallick, G. C. Trudel, P. Paliwal, S. Agrawal, and C. J. Logothetis. Dasatinib and docetaxel combination treatment for patients with castrationresistant progressive prostate cancer: A phase I/II study (CA180086), ASCO 2009 abstract 5061. (also presented as update at ESMO 2009).

18. AJ Armstrong, George DJ, and Halabi S. Serum lactate dehydrogenase (LDH) is a predictive biomarker for mTOR inhibition in patients with metastatic renal cell carcinoma (RCC). ASCO GU Symposium, San Francisco, CA 2010, abstract. Also: ASCO 2010, abstract 4631.

19. C.P. Hart, A.J. Armstrong, E.G. Chiorean, M. Borad, A. Mita, J.D. Sun, V.K. Langmuir, F. Meng, C. Eng, S. Kroll, M.D. Matteucci J.G. Curd. Bench to Bedside Experience with TH-302: a Tumor-Selective Hypoxia-Activated Prodrug as a Promising Treatment for Prostate Cancer. AACR-NCl-EORTC meeting November 2009, abstract.

20. Armstrong AJ, J. D. Turnbull, K. Morris, S. E. Yenser Wood, S. Voyles, Y. A. Fesko, and D. J. George. Impact of temsirolimus and anti-androgen therapy on circulating tumor cell (CTC) biology in men with castration-resistant metastatic prostate cancer (CRPC): A phase II study. ASCO 2010, abstract 47821.

21. Armstrong AJ, Oltean S, Kemeny G, Turnbull J, Herold C, Marcom PK, George DJ, Garcia-Blanco M, Circulating Tumor Cells from Patients with Metastatic Breast and Prostate Cancer Express Vimentin and $\mathrm{N}$-Cadherin. AACR Conference on EMT, Washington DC 2010, abstract and oral presentation.

22. Armstrong AJ, Oltean S, Kemeny G, Turnbull J, Herold C, Marcom PK, George DJ, Garcia-Blanco M, Plasticity, stemness, and aggressive behavior in preclinical models and circulating prostate cancer cells: importance of the transitional phenotypic state to lethal cancer biology. ASCO GU Symposium 2010, abstract 172 .

23. Sonpavde G, Pond GR, Berry WR, de Wit R, Armstrong AJ, Eisenberger M, Tannock IF. Changes in serum alkaline phosphatase predict survival independent of PSA changes in men with castration-resistant prostate cancer and bone metastasis receiving chemotherapy: a retrospective analysis of the TAX327 trial. ASCO GU Symposium 2010, abstract.34.

24. Pili R, Häggman RM, Stadler WM, Gingrich JR, Assikis V, Björk A, Forsberg G, Carducci MA, Armstrong AJ. A randomized multicenter international phase II study of tasquinimod in chemotherapy naïve patients with metastatic castrate-resistant prostate cancer (CRPC). ASCO 2010, abstract 4510. (oral presentation)

25. G. C. Trudel, F. Saad, A. J. Armstrong, J. Bellmunt, G. Wilding, E. Y. Yu, J. C. Araujo, S. Durham, P. Paliwal, C. Logothetis. Dasatinib or placebo combined with docetaxel in castration-resistant prostate cancer (CRPC): Design of CA180227, a phase 3, randomized, double-blind trial. ASCO 2011 abstract 80104

26. G. R. Pond, A. J. Armstrong, B. A. Wood, M. Brookes, L. H. Leopold, W. R. Berry, R. De Wit, M. A. Eisenberger, I. Tannock, G. Sonpavde. Evaluating the value of continuing docetaxel and prednisone [DP] beyond 10 cycles in men with metastatic castration resistant prostate cancer [mCRPC]. ASCO 2011 abstract 76830

27. M. T. Fleming, G. R. Pond, A. J. Armstrong, B. A. Wood, M. Brookes, L. H. Leopold, V. B. Matveev, J. M. Burke, J. R. Caton, G. Sonpavde. Ability of serum alkaline phosphatase [ALP] changes to complement PSA changes and predict survival in men with metastatic castration resistant prostate cancer [mCRPC] receiving docetaxel and prednisone [DP]. ASCO 2011 abstract 77014.

28. J. S. De Bono, K. Fizazi, F. Saad, M. E. Taplin, C. N. Sternberg, K. Miller, P. Mulders, K. Chi, A. Armstrong, M. Hirmand, B. Selby, H. I. Scher. Primary, secondary and quality-of-life endpoint results from the Phase 3 AFFIRM study of MDV3100, an androgen receptor signaling inhibitor. Proc ASCO 2012 
abstract 4519 .

29. Armstrong AJ, J.R. Gingrich, M. Häggman, W.M. Stadler, J.E. Damber, L. Belkoff, R. Clark, S. Brosman, O. Nordle, G. Forsberg, M.A. Carducci, R. Pili. Long term safety and efficacy in a randomized multicenter international phase II study of tasquinimod in chemotherapy naïve patients with metastatic castrateresistant prostate cancer. European Association of Urology, February 2012, Paris, abstract.

30. Armstrong AJ, R. Kaboteh, M.A. Carducci, J-E Damber, W.M. Stadler, M. Hansen, L. Edenbrandt, G. Forsberg, Ö. Nordle, R. Pili, M. Morris. Tasquinimod and effects on bone scan index in men with metastatic castration-resistant prostate cancer (mCRPC): results of retrospective follow up of a randomized phase 2 placebo-controlled trial. Proc Am Soc Clin Oncol 2013 abstract 5081.

31. Rhonda L. Bitting, Rengasamy Boominathan, Chandra Rao, Elizabeth Embree, Daniel J. George, Mark Connelly, Gabor Kemeny, Mariano A. Garcia-Blanco, and Andrew J. Armstrong. Isolation of Circulating Tumor Cells Using a Novel EMT-Based Capture Method. Proc Am Soc Clin Oncol 2013, abstract 5031.

32. Armstrong AJ, Halabi S, Eisen T, Stadler WM, Jones RR, Vaishampayan UN, Garcia JA, Hawkins RE, Kollmannsberger C, Lusk C, Broderick S, George DJ. ASPEN: A randomized phase II trial of everolimus versus sunitinib in patients with metastatic non-clear cell renal cell carcinoma. Proc Am Soc Clin Oncol 2013 abstract TPS 4590.

33. Higano C, Armstrong AJ, Cooperberg MR, Kantoff PW, Bailen J, Concepcion RS, Kassabian, Dakhil SR, Finkelstein SE, Vacirca JL, Rifkin RM, Sandler A, McCoy C, Whitmore JB, Tyler RC, Sartor AO. Impact of prior docetaxel (D) on sipuleucel-T (sip-T) product parameters in PROCEED patients (pts). Proc Am Soc Clin Oncol 2013, abstract 5034.

34. Scher HI, Fizazi K, Saad F, Chi KN, Taplin ME, Sternberg CN, Armstrong AJ, Hirmand M, Forer D, de Bono JS. Impact of on-study corticosteroid use on efficacy and safety in the phase III AFFIRM study of enzalutamide (ENZA), an androgen receptor inhibitor. Proc Am Soc Clin Oncol 2013, abstract 6.

35. TM Beer, AJ Armstrong, CN Sternberg, C Higano, $P$ Iversen, Y Loriot, DE Rathkopf, S Bhattacharya, J Carles, J de Bono, CP Evans, AM Joshua, C Kim, G Kimura, P Mainwaring, H Mansbach, K Miller, SB Noonberg, P Venner, B Tombal. Enzalutamide in Men with Chemotherapy-naïve Metastatic Prostate Cancer (mCRPC): Results of the Phase 3 PREVAIL Study. ASCO GU Symposium, San Francisco 2014, LBA1 abstract.

36. Armstrong AJ, Rhonda L Bitting, Gabor Kemeny, Daniel J George. Evidence for Circulating Tumor Cell (CTC) Alkaline Phosphatase (AP) Expression in Men with Bone-Metastatic CRPC During Abiraterone Acetate Treatment Response. ASCO GU Symposium, San Francisco, 2014, abstract 178.

37. Armstrong AJ, Beaver J, Li J, Bitting RL, Gregory S. Genomic Analysis of Circulating Tumor Cells (CTCs) from Men with Metastatic Castration Resistant Prostate Cancer (mCRPC) in the Context of Enzalutamide Therapy. ASCO GU Symposium, San Francisco, 2014, abstract 65.

\section{c: Editorials, position, and background papers}

1. Armstrong AJ, Eisenberger M. Commentary on: The risk of clinical fractures after gonadotropinreleasing hormone agonist therapy for prostate cancer. Nature Clin Pract Urol 2006; 3: 246-7.

2. Armstrong AJ, Garrett-Mayer ES, Eisenberger MA. Adaptive therapy for androgen-independent prostate cancer. J Natl Cancer Inst 2008;100:681-3.

3. Armstrong AJ, Moul JW, George DJ. What to order from the prostate cancer treatment menu? Oncology. 2012; 26:87-88. 
4. Sonpavde G, Armstrong AJ. Objective evaluation of bone metastases in prostate cancer: to what end? Eur Urol Epub July 2012.

5. Armstrong AJ. The STAMPEDE trial and celecoxib: how to adapt? Lancet Oncol 2012 Epub May 2012.

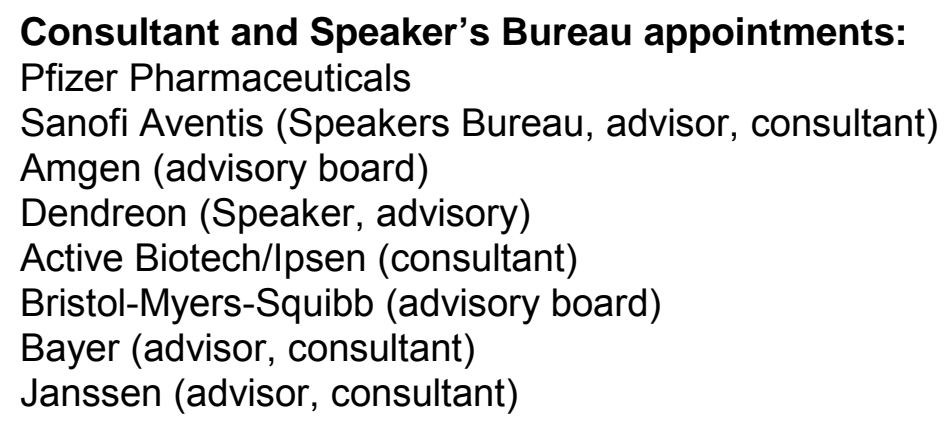

\section{Editorial Board Positions}

$\begin{array}{ll}\text { 2014-present } & \text { Journal of Clinical Oncology, } \\ \text { 2014-present } & \text { Prostate Cancer and Prostatic Diseases }\end{array}$

Technology Development:

1. Nomogram application (app) software. First smartphone-based software application for iphone or android for CRPC prognostic assessments, based on two Clin Cancer Res publications (2007, 2010) relevant to the pre- and post-docetaxel disease states. Launch: October 2013.

2. Patent for development of novel technology for circulating tumor cell capture based on EMT biology (application number PCT/US10/50223), patent pending.

\section{National/International Reviewer/Editorial Positions:}

\begin{tabular}{ll}
\hline 2014-present & Editorial board, Journal of Clinical Oncology \\
2013-present & Reviewer, New England Journal of Medicine \\
2013-present & Peer reviewer, American Urologic Association (AUA) guidelines \\
2011-present & Reviewer, Cancer Discovery \\
2011-present & Reviewer, PLoS One \\
2011-present & Prostate Cancer Foundation YIA Reviewer \\
2010-present & Reviewer, Lancet and Lancet Oncology \\
2010-present & Reviewer, Journal of Clinical Oncology \\
2010-present & Reviewer, BMC Cancer \\
2010-10-18 & Ad hoc Reviewer, Investigational New Drugs \\
2009-present & Scientific Editor, Prostate Cancer Foundation patient education webpage \\
2007-present & Reviewer, Cancer Investigation \\
2008-present & Reviewer, Clinical Advances in Hematology and Oncology \\
2007-present & Ad hoc reviewer, European Journal of Urology
\end{tabular}


2006-present

2006-present

2005-6
Reviewer, Prostate Cancer and Prostatic Diseases, ed. Judd Moul

Reviewer, Clinical Cancer Research, Cancer Research, and Molecular Cancer Therapy (AACR journals)

Faculty Reviewer, First Aid for the Boards, McGraw-Hill

Professional awards, National Committees, and Special Recognitions:

2014-present Prostate Cancer Foundation Global Research Council member

2012-

Fellow of the American College of Physicians (FACP)

2012-

Associate Director, Clinical and Translational Research in Genitourinary Oncology, Duke Cancer Institute

2012- $\quad$ ALLIANCE Cooperative Group, GU Correlative Science Committee member

2012-

Co-Director, Duke Scholars in Molecular Medicine, Oncology

$2011 \quad$ Medical Director, Duke Prostate Center Symposium

2010- $\quad$ NCCN Prostate Cancer Expert Panel Member (national guidelines)

2010-15 Department of Defense Physician Research Training Award, PCRP

2009-

2009

2008-11

2007-9

2007-9

2006-8

2006-9

Genitourinary Oncology co-Program Leader, Duke University

Prostate Cancer Foundation Top Performing Young Investigator

Prostate Cancer Foundation Young Investigator Award

National Comprehensive Cancer Network (NCCN)-mTOR inhibition in Solid Tumors Task Force Gold Star Service Champion, Duke University Medical Center, 9300 inpatient service

American Society of Clinical Oncology (ASCO) Young Investigator Award (YIA) American Association for Cancer Research (AACR) Clinical/Translational Research Fellowship

2006-9 Duke Comprehensive Cancer Center K12 Award

2006

2005-6

Cancer Education Consortium Grant Recipient, Amelia Island, Florida

2004

2003

2000

1999

1996

1996

Ad hoc reviewer, Urology (The Gold Journal), editor Alan W. Partin, MD PhD, Johns Hopkins Hospital, Baltimore MD.

1994-5 Summer research fellow at the National Cancer Institute's Advanced

Biosciences Laboratory (NCl-ABL) under Drs. George F. Vande Woude and Kenji Fukasawa

1995

1994

1993

K12 NIH Training Grant, Graduate Training Program in Clinical Investigation

National Medical Jeopardy Contestant, ACP National Convention, San Diego

Mulholland Society Teaching Award, University of Virginia

Alpha Omega Alpha

Tau Beta Pi National Engineering Honor Society

Graduation with Distinction in BME, magna cum laude

1992-6

Vice President, Duke University School of Engineering Student Body

Golden Key National Honor Society

Phi Eta Sigma National Honor Society

National Science Foundation Scholarship Award

Organizations and participation: (Offices held, committee assignments, etc.)

2012-present 2008-present 2008-present 2007-present 2007-present 2007-present 2006-present 2006-present 2006-present
Society for Urologic Oncology (SUO) member

Duke University Institutional Review Board

Scientific Editor, Duke Prostate Center News

Duke Comprehensive Cancer Center Editorial Advisory Committee

American Association for the Advancement of Science

NCCN mTOR inhibition in Solid Tumors Task Force

Duke Comprehensive Cancer Center Cancer Protocol Committee

Duke Clinical Research Institute Faculty Member

Cancer and Leukemia Group B, Active Member GU Committee 
2005-present 2003-present 2003-present 2000-present 1996-present 1995-6
American Association for Cancer Research (AACR), Active Member American Society of Clinical Oncology (ASCO), Active Member American Society of Hematology (ASH), Active Member American College of Physicians (ACP), Member American Medical Association, Member Vice President, Duke University School of Engineering Student Body

Teaching and lecturing responsibilities including continuing medical education (CME):

Jan 2014 Invited lecture, ASCO GU Symposium 2014: "Beyond Enzalutamide and Abiraterone: What's Next in Hormonal Therapy?"

2013-14 Multiple grand rounds on CRPC, Updates in Therapy

Oct 2013 Prognostic, Predictive, and Surrogate Biomarkers in CRPC, Labroots talk (CME)

Sept 2013 Update in CRPC, Dayton OH and separate grand rounds talk in Coumbia SC

April 2013 Prostate Cancer lecture, Duke Medicine Housestaff

Feb/April 2013 Medical Oncology Lectures on Prostate Cancer (2)

January 2013 Geriatrics Grand Rounds, lecture on Prostate Cancer in the Elderly

April 2013 Prostate Cancer lecture, Duke Medicine Housestaff

October 2012 Lecture on Epithelial Plasticity in Prostate Cancer, Prostate Cancer Foundation Retreat, Carlsbad, CA

October 2012 Prognostic and Predictive Biomarkers in CRPC, SUO Symposium Fukuoka, Japan

7/20/2012 Best of ASCO Highlights, Cary NC

2010-12 Lunch and learn series: topics in GU Oncology (monthly lectures to research staff)

5.2012 Updates in CRPC; William J Smith Memorial Oncology Conference, Asheville, NC

4.2012 Oncology Grand Rounds: Duke Debate

4.2012 Updates in CRPC Podcast, CancerNetwork

2.2012 Novel therapies for CRPC: EAU Invited Lecture, Paris France

2012 RCC CME Program, France Foundation (Duke CME Program): includes podcasts, presentations, interviews, development of slide deck

01.2011 2011 Testicular Cancer Lecture and 2011 Prostate Cancer Lecture

11.2010 Talk entitled: "CRPC: What Else is Out There?" for the UK Cancer Convention, Royal Institute of British Architects (RIBA), London

9. 2010 Oncology Care Live 2010 Virtual Oncology Congress, speaker 
Medical Oncology Grand Rounds: A randomized multicenter international phase II study of tasquinimod in chemotherapy naïve patients with metastatic castrate-resistant prostate cancer (CRPC).

2009 Medical Oncology Grand Rounds: "Epithelial Plasticity in Prostate Cancer: A Biomarker for the Lethal Phenotype"

1.2010 CALGB Duke/Duke Oncology Network CRA Workshop

4.2008 Duke Prostate Center and Duke Urologic Assembly, Prostate Cancer Update: "Complications of Androgen Deprivation Therapy"

2009 Duke Oncology Network ASCO Updates

2008 Duke Tuesdays in Urology: "Update in Castration Resistant Prostate Cancer"

10.2007 "Current Directions in Advanced Prostate Cancer Therapy", Fall Oncology Conference, Birmingham, AL

5.2007 Updates in Prostate Cancer, 2007. Nash General Hospital, Rocky Mount, NC

3.2007 Update in Advanced Prostate Cancer. Duke Prostate Center Symposium, Durham, NC

2006-8 Attending and Teaching physician, 9300 solid tumor inpatient unit, Duke University Hospital

2007-pres. Fellow lecture series, medical oncology, Duke University

2006-7 Teaching physician, mock tumor board, Duke University School of Medicine

2006 Attending and Teaching Physician, 9300 Inpatient Service in Medical Oncology

2003-6 Fellow in oncology and hematology with teaching responsibilities for Johns Hopkins medical housestaff: inpatient services and consultative services

2000

Mulholland Society Teaching Award, University of Virginia School of Medicine

\section{Mentoring Responsibilities:}

1. Primary clinical and translational mentor for Jing Li, MD PhD, $2^{\text {nd }}$ year post-doctoral medical oncology fellow. Project: " Novel mesenchymal capture and genomic characterization of CTCs in men with mCRPC".

2. Primary clinical and translational mentor for Tian Zhang, MD, $2^{\text {nd }}$ year medical oncology post-doctoral fellow, project entitled "The role of c-met in promoting AR independent prostate cancer growth", and "Development of a novel CTC capture method based on c-met expression."

3. Kathryn Ware, PhD. Post-doctoral fellow. Clinical and translational co-mentor along with Mariano Garcia-Blanco. Project: "Association between AR variants and epithelial plasticity in CRPC."

4. Rhonda Bitting, MD (Oncology Fellow). Project is around developing methods for the analysis of RNA expression profiles in circulating tumor cells from men with metastatic CRPC. Co mentor is Mariano Garcia-Blanco. 2010-present 
5. Abhinav Ettyreddy (Duke Undergraduate). Third year project for thesis work dedicated to development of FGFR2 isotype specific antibodies for use in tissue and circulating tumor cell research. Co mentor is Mariano Garcia-Blanco. 2010-present

6. Clinical/translational mentor for post-doctoral T32 fellows in Mariano Garcia-Blanco laboratory: Daneen Schaeffer PhD, Matthew Marengo PhD, Jason Somarelli PhD, 2010-present

7. Clinic Mentor for medical oncology fellows in GU. Prateek Mendiratta and Franklin Chen. 2008-2009.

8. Clinic mentor for several undergraduate students: Sarah Wang, Geoffrey Houtz, 2012-present

9. Clinical/translational mentor for Molecular Medicine Scholars in Oncology, 2011-present

\section{Areas of research interests (basic and applied) - list:}

1. Predictors of sensitivity and clinical efficacy of mTOR inhibitors in advanced prostate cancer

2. Novel designs of clinical trials and pharmacodynamic/translational studies in GU malignancies

3. Pre-operative models for drug development of novel agents in human testing in prostate cancer

4. Novel therapies and drug development for prostate, renal, and bladder cancer

5. Design of rational combination therapies with mTOR inhibition in metastatic castration-resistant prostate cancer

6. Exploration of resistance mechanisms to rapamycin and its analogues in prostate cancer

7. Developing prognostic models for progression and survival in metastatic hormone-refractory prostate cancer

8. Examining surrogate markers of mortality in metastatic castration-resistant prostate cancer

9. Non-clear cell renal cell carcinoma novel therapeutic strategies

10. Circulating tumor cell biology and genomics/genetics for personalized medicine approaches to CRPC

\section{Current Projects and Studies}

See other support page 
External support - gifts, grants, and contracts: see other support page for details

PI $\quad$ E Effort $\quad \underline{\text { Purpose }} \quad \begin{aligned} & \text { Approximate } \\ & \text { Amount }\end{aligned}$

\section{Past:}

Johns Hopkins K12

(Institutional)

Ross

n/a $\quad$ GTPCl Training

$\$ 60,000 /$ year

2004-6

Grant (Fellowship)

Duke University K12 H. Kim Lyerly

(Institutional K12, DCCC)

$75 \% \quad$ Salary Support $\quad \$ 115,000 / y r$

2006-9

GCRC

(Johns Hopkins)

Carducci, MA

$\mathrm{n} / \mathrm{a}$

Rapamycin Study

$\$ 15,000$

2006-7

ASCO YIA

Armstrong AJ

$\mathrm{n} / \mathrm{a}$

Rapamycin Study

$\$ 36,000$

2006-8

(Young Investigator Award)

AACR

$$
\text { Armstrong AJ n/a }
$$

(Clinical and Translational Fellowship Grant)

Rapamycin Study $\$ \$ 40,000$

2006-8

Present (see other support page):

$\begin{array}{llclcr}\begin{array}{l}\text { Prostate Cancer } \\ \text { Foundation }\end{array} & \text { Armstrong AJ } & 1-5 \% & \begin{array}{l}\text { Epithelial Plasticity } \\ \text { in Prostate Cancer }\end{array} & \$ 75,000 / \mathrm{yr} & 2008-11 \\ \text { DOD PRTA } & \text { Armstrong } & 53 \% & \begin{array}{l}\text { Epithelial Plasticity } \\ \text { In CRPC }\end{array} & \$ 685,172 & 2010-2015 \\ \text { DOD NIA } & \text { Armstrong } & 10 \% & \text { Polymersomes } & \$ 291,000 & 2012-14 \\ \text { NIH R01 } & \text { Garcia-Blanco } & 5 \% & \text { Epithelial plasticity } & \text { see OS } & 2008-13\end{array}$

Multiple clinical trial awards (see other support page)

Department of Defense Prostate Cancer Clinical Trials Consortium Grant (DOD PCCTC), sub-investigator, $5 \%, 5$ years $2009-2014$. 


\section{Clinical activity - type of practice and estimate of time commitment:}

2006-2012 Assistant Professor, Duke University Department of Medicine, Division of Medical Oncology, $33 \%$ effort for direct patient care

2012-present Associate Professor of Medicine and Surgery, Duke Cancer Institute. 33\% effort for patient care.

Recognition as an international leader in experimental therapeutics in prostate cancer, biomarker development in GU malignancies, prognostic and predictive biomarkers. Practice includes one physician assistant, Kristen Davis PA, who follows patients with me during systemic treatments for GU cancers.

Currently spend one half day per month additional clinic session in the Duke Multidisciplinary Prostate Cancer Clinic for newly diagnosed men, 2010-present.

2013-present Associate Director for Clinical Research, Genitourinary Program, Duke Cancer Institute, 200 hours/year

Duties: 1) oversee staff of 8 research coordinators, 3 regulatory coordinators, 3 data managers, 2 clinical trial assistants, finance (pre and post-award) personnel; 2) hold regular weekly data and safety monitoring meetings related to clinical trial patient care; 3 ) lead weekly new protocol meetings to develop new ideas, grants, processes, database studies, clinical trials

2013-present Member, Prostate Cancer Strategy Group, Duke Cancer Institute, monthly meetings Goal: To work with multidisciplinary team to set strategic goals around prostate cancer screening, detection, diagnosis, risk stratification, management, and to develop recruitment priorities for the $\mathrm{DCl}$.

Developed prostate cancer screening MAESTRO template for use in all Duke primary care clinics.

\section{Participation in academic and administrative activities of the University and Medical Center:}

2013-present Duke Cancer Institute Prostate Cancer Strategy Group

2013-present Associate Director for Clinical Research, GU Program, DCl

2010-present Duke Comprehensive Cancer Center Oncology Trials Shared Resource, co-GU Program Lead

2009-present Duke University Department of Medicine Residency Interviewing Committee

2010-present Duke Fellowship Advisory Committee

2008-present Member, Duke University Institutional Review Board

2008-present Internal Medicine residency interviewing committee

2007-present Duke Comprehensive Cancer Center Editorial Advisory Committee

2007-present Senior Scientific Editor, Duke Prostate Center News (periodical)

2006-present Fellowship recruitment and interviewing committee, Duke University Department of Medicine

2006-present Cancer Protocol Committee (CPC), Duke Comprehensive Cancer Center 


\section{Research Support}

\section{Completed}

AACR Clinical/Translational Fellowship (Armstrong)

$07 / 01 / 2006-06 / 30 / 2008$ AACR

A Pharmacodynamic Study of Pre-Prostatectomy Rapamycin in Men with Advanced Localized Prostate Cancer.

Goal: Project was to determine the safety and optimal target dose of the oral mTOR inhibitor rapamycin when administered daily as a single agent to men with localized high-risk prostate cancer prior to undergoing radical prostatectomy and to identify predictors of pharmacodynamic response to rapamycin using tissue-based mechanistic studies in locally advanced prostate cancer.

\section{American Society of Clinical Oncology Young Investigator Award (YIA)} 7/01/06-6/30/2008

ASCO: PI Andrew J. Armstrong, MD

A Pharmacodynamic Study of Pre-Prostatectomy Rapamycin in Men with Advanced Localized Prostate Cancer.

Goal: This grant funds some of the correlative science work for this clinical trial, including pharmacokinetics and pathologic/immunohistochemical assessments.

NIH 5K12 CA100639 (Lyerly, H.)

$$
\text { 08/04/2004 - 07/31/2009 }
$$

NIH Clinical Oncology Research Career Development Program (Armstrong)

Goal: Dr. Armstrong was supported over a 3-year term to conduct a trial which includes the following specific aims: (1) to evaluate the safety and tolerability of the oral mTOR inhibitor rapamycin when administered daily as a single agent to men with localized intermediate and high-risk prostate cancer prior to undergoing radical prostatectomy; (2) to determine the POD of rapamycin in men with newly diagnosed, intermediate and highrisk prostate cancer using prostatic tissue collected at the time of prostatectomy; and (3) to identify predictors of biologic response to rapamycin using tissue-based mechanistic studies in locally advanced prostate cancer.

\section{(Armstrong)}

$$
08 / 01 / 08-07 / 31 / 11
$$

\section{Bristol-Myers Squibb}

Phase I/II Study of Dasatinib and Docetaxel in Metastatic Hormone Refractory Prostate Cancer Goal: Phase II portion of this clinical research study is to learn how the study drugs (dasatinib, docetaxel, and prednisone) affect each other in the body

07TASQ08 (Armstrong)

$$
\text { 01/02/08 - 01/02/11 }
$$

\section{Active Biotech AB}

Phase II Randomized Double Blind Placebo-Controlled Study to Determine Efficacy of ABR-215050 Goal: Evaluate the efficacy of ABR-215050 vs. placebo in asymptomatic patients with metastatic CRPC, as measured by the proportion of patients who have not progressed at 6 months and to evaluate the effect of ABR-215050 vs. placebo in asymptomatic patients with metastatic CRPC 
Goal: The purpose of this study is to evaluate the effects of IMC-A12 or IMC-1121B combined with Mitoxantrone and Prednisone in patients with metastatic prostate cancer on progression free survival.

\section{Current/Ongoing/Active}

Department of Defense W81XWH-10-1-0483 (Armstrong) 07/01/10 - 07/31/15 Epithelial Plasticity in Castration-Resistant Prostate Cancer: Biology of the Lethal Phenotype Goal: Investigate the prevalence of epithelial plasticity and stem cell biomarkers on CTCs; identify oncogenic pathways through RNA expression profiling that are activated in CTCs compared to matched leukocytes and metastatic tumor samples; investigate the clonality of prostate cancer metastases through analysis of DNA copy number changes in matched CTCs and metastatic sites.

Department of Defense W81XWH-12-1-0253 (Armstrong)

09/10/12 - 09/09/14

Development of a Novel Method to Detect Prostate Cancer Circulating Tumor Cells (CTCs)

Goal: Develop and optimize a novel polymersome-based CTC capture method using NIR-EPs bearing conjugated antibodies to EpCAM, N and O-cadherins, and PSMA; Assessment of CTC capture using novel antibody-targeted NIR-EPs in men with mCRPC; long term goal of this DOD IDA/NIA is to develop a noninvasive strategy for detection and characterization of non-epithelial CTCs to improve upon and complement existing epithelialbased CTC detection technology, and identify novel CTC populations and thus therapeutic targets to prevent or delay metastatic progression in men with PC.

National Institutes of Health 5R01-CA127727-05 (Garcia-Blanco)

$12 / 01 / 08-11 / 30 / 13$

Alternative Splicing and Epithelial-mesenchymal Plasticity in Prostate Tumors

Goal: Investigate the mechanisms involved with the alternative splicing of FGFR2 in prostate cancers as they transition from an epithelial phenotype to a mesenchymal phenotype.

National Institutes of Health 5R01-CA155296-03 (Halabi)

07/06/11 - 05/31/15

\section{Prognostic Models of Clinical Outcomes In Men With Castration Resistant Prostate Cancer}

Goal: Develop a prognostic model that will predict overall survival in men with CRPC who failed first line chemotherapy. The model will be validated for predictive accuracy using an independent dataset; develop a prognostic model that will predict progression-free survival in CRPC men who failed first line chemotherapy.

Prostate Cancer Foundation (Armstrong)

Epithelial Plasticity in Advanced Prostate Cancer: A Biomarker for Lethal Disease

09/01/08 - 08/31/13

Goal: Identify CTCs in patients with PC with this aggressive mesenchymal/pro-metastatic phenotype that are not currently identified in existing assays.

\section{INDUSTRY SPONSORED PROJECTS}

Aggregated Effort

2.16 calendar

Duke University lists aggregated effort assigned to the following eligible industry-sponsored clinical trial projects. Each of these individual projects has a varying need of effort depending on the type of activity currently in progress such as protocol development, start-up, patient recruitment, enrollment, follow-up, monitoring, data analysis, publication, and closeout. Faculty determines each project's need and adjust their effort between projects within the total aggregated effort assigned to the clinical projects.

Novartis Pharmaceuticals Corporation (Armstrong)

11/05/09 - 02/29/16 A Randomized Phase II Study of Afinitor (RAD0001) vs. Sutent (Sunitinib) in Patients with Metastatic NonClear Cell Renal Carcinoma (ASPEN)

Goal: Compare the anti-tumor activity of RAD001 and sunitinib in subjects with metastatic renal cell carcinoma (mRCC) with non-clear cell pathology, as measured by progression free survival (PFS) following treatment initiation. 
A Randomized Double-Blind Phase III Trial Comparing Docetaxel Combined with Placebo in Castrationresistant Prostate Cancer

Goal: Compare overall survival for dasatinib plus docetaxel and prednisone versus placebo plus docetaxel and prednisone in subjects with metastatic castration-resistant prostate cancer; compare the rate of change from baseline in urinary $\mathrm{N}$-telopeptide between the 2 treatment arms; compare the time to first skeletal related event between the 2 treatment arms; compare the rate of change from baseline in pain intensity between the 2 treatment arms; compare the time to PSA progression between the 2 treatment arms.

Active Biotech AB (Armstrong)

$03 / 23 / 11-03 / 31 / 14$

A Phase III Randomized Double-blinded Placebo Controlled Study of Tasquinimod in Men with Metastatic Castrate-resistant Prostate Cancer

Goal: To confirm the effect of tasquinimod on delaying disease progression compared with placebo.

Medivation, Inc.MDV3100-09 (Armstrong)

STRIVE: A Multicenter Phase II, Randomized, Double-Blind, Efficacy and Safety

$01 / 10 / 2013-12 / 31 / 14$

Goal: Determine the benefit of enzalutamide (formerly MDV3100) as compared to bicalutamide as assessed by progression-free survival (PFS).

Novartis Pharmaceuticals Corporation (Armstrong)

01/01/11 - 12/31/13

A Randomized Phase II Study of BKM120 in Men with Castration-resistant Metastatic Prostate Cancer

Goal: Evaluate the effects of the study drug BKM120 and changes in response to BKM120.

Pfizer, Inc. (Armstrong)

09/10/09 - 12/31/13

A Randomized Phase II Study of Afinitor (RAD001) vs. Sutent (Sunitinib) in Patients with Metastatic Non-Clear Cell Renal Cell Carcinoma

Goal: Compare the anti-tumor activity of RAD001 and sunitinib in subjects with metastatic renal cell carcinoma (mRCC) with non-clear cell pathology, as measured by progression free survival (PFS) following treatment initiation.

Pfizer, Inc (Armstrong)

$12 / 01 / 08-11 / 30 / 13$

Multimodality therapy for recurrent high-risk prostate cancer, a phase 2 trial

Goal: To determine the rate of progression free survival (PFS) at 24 months in men with PSA recurrent nonmetastatic prostate cancer following radical prostatectomy who received multimodality therapy.

Medivation, Inc. (Armstrong)

$10 / 05 / 10-11 / 11 / 13$

A Multinational Phase 3, Randomized, Double-blind, Placebo-Controlled Efficacy and Safety Study of Oral MDV3100 in Chemo-Naïve Men with mCRPC

Goal: Determine the benefit of MDV3100 as compared to placebo as assessed by overall survival and to determine the benefit of MDV3100 as compared to placebo as assed by progression-free survival (PFS).

KangLaiTe USA Inc (Armstrong)

Efficacy and Safety of oral Kanglaite (KLTc) Gelcap in Men with Prostate Cancer

08/01/11 - 09/30/13

Goal: Evaluate the effects and safety of two different doses (3 or 6 capsules, four times daily) of KLTc on prostate specific antigen doubling time (PSADT) in men who have rising PSA after initial local therapy for localized prostate cancer during 12 months of study period.

Dendreon Corporation (Armstrong)

09/18/09-08/31/13

An Open-Label Study of Sipuleuciel-T in Men with Metastatic Castrate resistant Prostate Cancer

Goal: Provide sipuleucel-T to men with metastatic castrate resistant prostate cancer (CRPC), while marketing approval is being pursued; obtain safety data, to evaluate the magnitude of immune responses to treatment with sipuleucel-T, and to further characterize the cellular components of sipuleucel-T.

Dendreon Corporation Protocol P10-3 (Armstrong)

A Registry of Sipuleucel-T Therapy in Men with Advanced Prostate Cancer

$07 / 11 / 11-08 / 01 / 13$ 
Goal: This is strictly an observational study. The patient will receive sipuleucel-T and the study doctor will observe and collect information about the patient. 